\title{
A TRAJECTORY APPROACH TO ANALYZING THE INGREDIENTS ASSOCIATED WITH HEAVY WINTER STORMS IN CENTRAL NORTH CAROLINA
}

\author{
Christopher M. Fuhrmann
}

A dissertation submitted to the faculty of the University of North Carolina at Chapel Hill in partial fulfillment of the requirements for the degree of Doctor of Philosophy in the Department of Geography (Climatology)

Approved by:

Advisor: Charles E. Konrad II

Reader: John Bane

Reader: Scott Curtis

Reader: Peter J. Robinson

Reader: J. Marshall Shepherd

Reader: Thomas Whitmore 
(C) 2011

Christopher M. Fuhrmann

ALL RIGHTS RESERVED 


\begin{abstract}
CHRISTOPHER M. FUHRMANN: A Trajectory Approach to Analyzing the Ingredients Associated with Heavy Winter Storms in Central North Carolina

(Under the direction of Charles E. Konrad II)
\end{abstract}

Winter storms, namely snowstorms and ice storms, are a major hazard and forecasting challenge across central North Carolina. This dissertation employs a trajectory approach to analyze the ingredients (i.e. temperature, moisture, and lift) associated with heavy snowstorms and ice storms that occurred within the Raleigh, NC National Weather Service forecast region from 2000 to 2010. The Hybrid Single-Particle Lagrangian Integrated Trajectory (HYSPLIT) tool is used to a calculate 72-hour backward (i.e. upstream) air parcel trajectories from three critical vertical pressure levels at the time and location of heaviest precipitation for each storm. Analysis of composite trajectories reveals the source regions and meteorological properties of air parcels associated with heavy winter storms. Adiabatic and diabatic contributions to air parcel temperature and moisture content are also calculated along each trajectory to assess the physical processes connected with heavy winter precipitation in the region. Comparative and ensemble trajectories are calculated to determine the physical processes that distinguish between precipitation type and intensity and assess the sensitivity of the trajectory calculations to the meteorological input data. A synoptic analysis of geopotential height fields is undertaken to determine those aspects of the large-scale circulation responsible for the back trajectory motions. Results indicate that diabatic warming and cooling contribute significantly to the vertical temperature profile 
during heavy winter storms and therefore dictate the resulting precipitation type. The main source of diabatic warming is fluxes of sensible and latent heat within the marine atmospheric boundary layer over the Gulf Stream. These fluxes contribute to a warming and moistening of air parcels associated with heavy ice storms. In contrast, heavy snowstorms are characterized by diabatic cooling in the lower troposphere above the marine atmospheric boundary layer. The most significant moisture source for heavy snowfall is the Caribbean Sea, while heavy ice storms entrain moisture from the Gulf of Mexico and Gulf Stream region near the Carolina coast. The details of the trajectory motions and air parcel characteristics have a significant influence on precipitation type and intensity and in many cases these details can be tied to subtle differences in the evolution of the synoptic-scale circulation. 
To Kristin 


\section{ACKNOWLEDGEMENTS}

I wish to thank my committee - Chip Konrad, Peter Robinson, Scott Curtis, Marshall Shepherd, John Bane, and Tom Whitmore - for their support and feedback throughout this dissertation project. I also wish to thank Brandon Locklear, Jonathan Blaes, and Gail Hartfield of the Raleigh National Weather Service for their insights on the challenges of forecasting winter precipitation in central North Carolina. I thank Baker Perry and John Cortinas for their insights on winter precipitation processes. Baker Perry also provided feedback on the design of the trajectory analysis. Lastly, I want to extend a special thankyou to my advisor, Chip Konrad, who provided unwavering guidance and encouragement, and was incredibly patient with me, particularly over the final year of the project while I worked full-time for the Southeast Regional Climate Center. 


\section{TABLE OF CONTENTS}

LIST OF TABLES ......................................................................

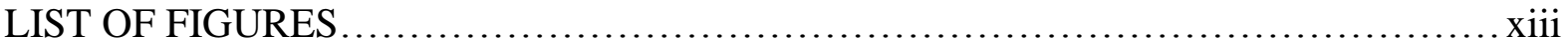

\section{Chapter}

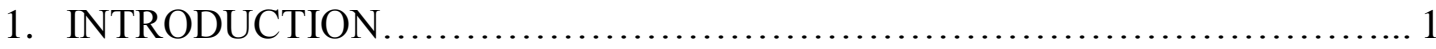

Research Context..................................................... 1

Literature Synthesis....................................................

The Ingredients Required for Winter Precipitation....................4

Winter Precipitation Types.................................... 5

Definitions and Distributions................................ 5

Physical Processes Affecting Precipitation Type.............11

Using Partial Thickness to Forecast Precipitation Type........ 13

Synoptic-Scale Features Associated with Winter Precipitation........ 16

Subsynoptic-Scale Features and Processes

Associated with Winter Precipitation.............................. 22

Cold-Air Damming ....................................... 22

Upstream Convection and Instability....................... 24

The Conveyor Belt Model......................................26

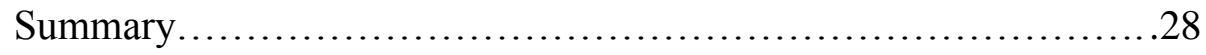

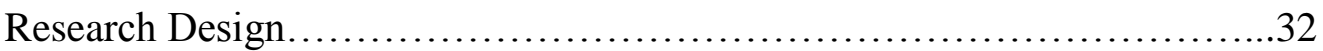


Research Questions......................................... 32

Research Objectives.......................................33

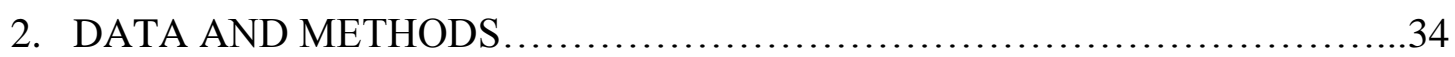

Overview.............................................................

Study Area....................................................... 34

Selection of Winter Storms........................................ 36

Trajectory Analysis........................................... 40

Theory................................................. 40

Limitations.................................................. 43

HYSPLIT Description....................................46

Meteorological Dataset................................... 47

Research Methods...............................................49

Model Soundings......................................... 49

Trajectory Calculations....................................53

Synoptic Analysis....................................... 57

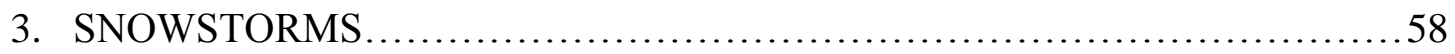

Overview.........................................................

Modeled Vertical Profile.............................................59

Trajectory Analyses............................................ 64

Composite Trajectories...................................6 64

Comparative and Ensemble Trajectories....................... 76

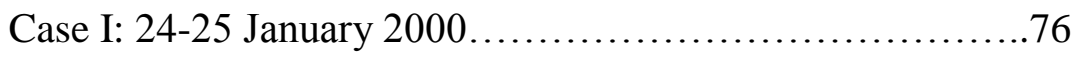

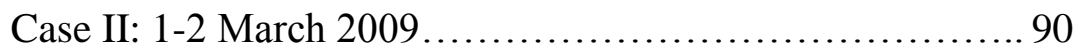


Synoptic Analyses .104

Near-Surface Trajectory......................................104

Warm Layer Zone Trajectory........................... 105

Dendritic Growth Zone Trajectories.............................. 106

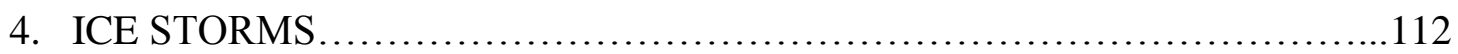

Overview..................................................... 112

Modeled Vertical Profile................................................ 112

Trajectory Analyses............................................. 118

Composite Trajectories.......................................118

Comparative and Ensemble Trajectories......................130

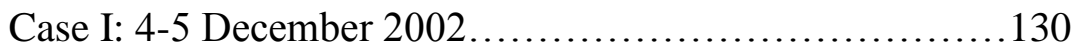

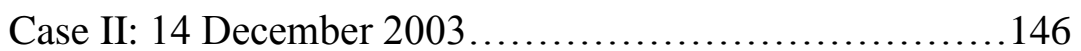

Synoptic Analyses............................................. 158

Near-Surface Trajectory....................................158

Warm Layer Zone Trajectory..............................159

Dendritic Growth Zone Trajectories.............................. 160

5. DISCUSSION AND CONCLUSIONS .................................. 168

Overview.................................................... 168

Major Findings................................................. 169

Vertical Profile.............................................. 169

Trajectories...........................................170

Near-Surface Trajectory..............................170

Warm Layer Zone Trajectory.........................171 


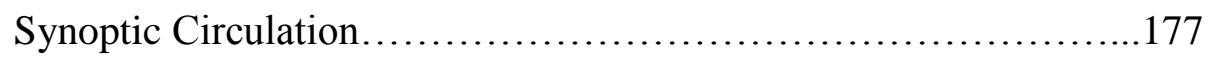

Comparisons to the Conveyor Belt Model.............................179

Future Research..................................................... 184

APPENDIX I: Precipitation Maps...................................... 189

APPENDIX II: Comparison of Observed and Modeled Soundings..............198

APPENDIX III: Composite Trajectories with Range Bars................... 200

REFERENCES................................................ 212 


\section{LIST OF TABLES}

Table

Page

2.1 List of snowstorms examined in this study. Locations for the sounding

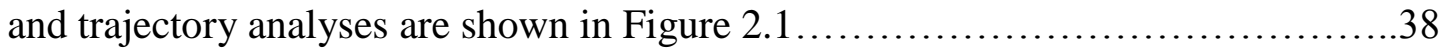

2.2 List of ice storms examined in this study. Locations for the sounding and trajectory analyses are shown in Figure 2.1. Values in italics are for combined sleet and freezing rain totals................................................ 38

2.3 Example of the procedure used to identify the time of heaviest precipitation. The 3 -hr period with the heaviest aggregate precipitation ( 0.33 in liquid equivalent) is highlighted in the Precipitation column with the middle hour (07 UTC) highlighted in the Time column...

3.1 Modeled vertical temperature $\left({ }^{\circ} \mathrm{C}\right)$ profile for each snowstorm. Temperatures associated with the dendritic growth zone are highlighted in gray. The maximum temperature in the low-level inversion is underlined........61

3.2 Same as Table 3.1, except for the modeled vertical moisture profile $(\mathrm{g} / \mathrm{kg}) \ldots \ldots \ldots . .62$

3.3 Same as Table 3.1, except for the modeled mean instantaneous vertical velocity $(\mathrm{cm} / \mathrm{s})$. Negative (positive) values indicate upward (downward) vertical motion.

3.4 Hourly snowfall totals (in liquid equivalent) for the 24-25 January 2000 snowstorm at RDU, INT, and RWI. Values in brackets indicate periods of rain. Back trajectories were computed from the time highlighted in boldface

3.5 Hourly snowfall totals (in liquid equivalent) and present weather observations for the 1-2 March 2009 snowstorm at GSO. Back trajectories were computed from the times highlighted in boldface.

4.1 Modeled vertical temperature $\left({ }^{\circ} \mathrm{C}\right)$ profile for each ice storm. Temperatures associated with the dendritic growth zone (warm layer zone $>0{ }^{\circ} \mathrm{C}$ ) are highlighted in gray (red). The maximum temperature in the warm layer zone is underlined.

4.2 Same as Table 4.1, except for the modeled vertical moisture profile $(\mathrm{g} / \mathrm{kg})$

4.3 Same as Table 4.1, except for the modeled mean instantaneous vertical velocity $(\mathrm{cm} / \mathrm{s})$. Negative (positive) values indicate upward (downward) vertical motion. 
4.4 Hourly precipitation totals (in. liquid equivalent) and present weather for the 4-5 December 2002 ice storm at RDU and GSO. Back trajectories were computed from the time highlighted in boldface.

4.5 Hourly precipitation totals (in. liquid equivalent) and present weather for the 14 December 2003 ice storm at RDU and GSO. Back trajectories were computed from the time highlighted in boldface. 


\section{LIST OF FIGURES}

Figure

Page

1.1 Schematic map illustrating the regional topography and the main cyclone tracks (L) and air masses connected with winter precipitation in central North Carolina (reproduced from Keeter et al. 1995) .3

1.2 Schematic showing the vertical thermal profiles associated with (A) freezing rain, (B) sleet, (C) snow, and (D) snow with an isothermal layer resulting from diabatic cooling. The dotted vertical line represents the $0^{\circ} \mathrm{C}$ isotherm and the thick line represents the environmental lapse rate.

1.3 Examples of winter storms in North Carolina associated with (A) a narrow transition zone of freezing rain (bottom image) juxtaposed with a region of snow to the northwest (top image) and (B) a broad transition zone of mixed precipitation across most of central North Carolina. Figures produced by the Raleigh NWS forecast office.

1.4 Partial thickness nomogram for Greensboro, NC (GSO) produced by the Raleigh NWS forecast office. Thickness values given in meters

1.5 Example of a "Gulf Atlantic" or Miller-A cyclone type at (A) 12 UTC 24 January 2000 and (B) 24 hours later (12 UTC 25 January 2002)...

1.6 Example of an "Atlantic Coast Redevelopment" or Miller-B cyclone type at (A) 12 UTC 4 December 2002 and (B) 24 hours later (12 UTC 5 December 2002).

1.7 Surface weather map showing a well-defined cold-air damming (CAD) signature along the U.S. East Coast. ...

1.8 Surface analysis of frontal boundaries and precipitation during a severe ice storm (top) and corresponding pattern of cloud-to-ground lightning flashes (bottom). The general region of convection is highlighted in both images by red ovals. The mean steering flow is indicated with red arrows. This suggests that ice from the tops of the convective clouds were advected downwind, helping to enhance precipitation rates north of the frontal boundary. From Hunter et al. (2001), their Figure 3. Red ovals and arrows added for illustration.

1.9 Schematic representation of the primary conveyor belts in a mature extra-tropical cyclone (from $\mathrm{Ng} 2005$, his Figure 7.9)

1.10 Depiction of the anticyclonic path of the cold conveyor belt using isentropic surfaces (solid black streamlines) (from Carlson 1980, his Figure 4). 
2.1 County-level map of North Carolina with the Raleigh NWS County Warning Area highlighted in yellow. Stars indicate the first-order stations and starting locations (with labels) for the sounding and trajectory analyses

2.2 A set of 5-day backward trajectories illustrating an intense water vapor transport event into western Canada. Coloring represents various parameters calculated along each trajectory segment: (top) pressure (hPa); (middle) relative humidity (percent); and (bottom) equivalent potential temperature (K). From Roberge et al.

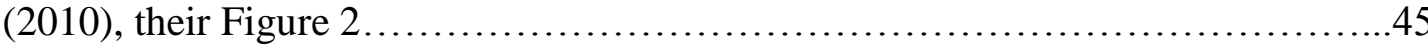

2.3 The EDAS grid domain displayed in Google Maps.............................48

2.4 Percentage of the total precipitation observed across different time periods relative to the full duration of each (A) snowstorm and (B) ice storm

2.5 Example of the procedure used to calculate adiabatic and diabatic contributions to the change in temperature of an air parcel between two trajectory segments $\left(\mathrm{t}_{0}\right.$ and $\left.\mathrm{t}_{0}+1\right)$.

3.1 Horizontal trajectory composites of air parcels terminating at the near-surface, warm layer zone, and dendritic growth zone in the region of heavy snowfall.

3.2 Vertical trajectory composites of air parcels terminating at the near-surface, warm layer zone, and dendritic growth zone in the region of heavy snowfall. Composites with range bars can be found in the appendix

3.3 Same as Figure 3.2, except for composite trajectories of air parcel temperature.....71

3.4 (A) Same as Figure 3.2, except for moisture composites along each trajectory. (B) Schematic illustration of the moisture curve representing a typical evaporation-precipitation cycle where " $\mathrm{E}$ " is the primary period of evaporative increase in humidity leading up to the final period of precipitative loss "P" of humidity. From James et al. (2004), their Figure 10

3.5 Same as Figure 3.2, except for composite trajectories of air parcel potential temperature.

3.6 Net diabatic contribution to air parcel temperature at each critical level in the region of heavy snowfall. Range bars indicate the maximum and minimum values among all eight snowstorms 
3.7 Warm layer zone trajectories of observed air parcel temperature (black line), adiabatic temperature (thick blue line), and diabatic contribution (gray bars) in the region of heavy snowfall at 6-hr trajectory segments. Range bars indicate the maximum and minimum values among all eight snowstorms. .... .75

3.8 Horizontal and vertical trajectories for 06 UTC 25 January 2000 terminating at the near-surface level (red trajectories), WLZ (blue trajectories), and DGZ (green trajectories) at (A) Raleigh-Durham (RDU), (B), Rocky Mount (RWI), and (C) Winston-Salem (INT). Vertical trajectories are shown at the bottom

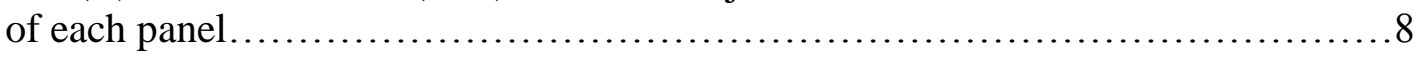

3.9 Air parcel trajectories of (A) potential temperature and (B) moisture content for 06 UTC 25 January 2000 terminating in the DGZ at RDU, RWI, and INT.

3.10 Backward ensemble trajectories for 06 UTC 25 January 2000 calculated from RDU at the near-surface level. Vertical trajectories are shown in the bottom panel.

3.11 Backward ensemble temperature trajectories for 06 UTC 25 January 2000 calculated from RDU at the near-surface level..................................84

3.12 Same as Figure 3.10, except at the WLZ .................................... 85

3.13 Same as Figure 3.11, except at the WLZ .................................... 86

3.14 Net diabatic contribution to air parcel temperature trajectories at RDU with WLZ temperatures (A) above freezing and (B) below freezing at 6-hr trajectory segments. Range bars indicate the maximum and minimum values among the ensemble members

3.15 Same as Figure 3.10, except at the DGZ ..................................... 88

3.16 Backward ensemble trajectories of moisture content for 06 UTC 25 January 2000 calculated from RDU at the DGZ with origins over the (A) Atlantic Ocean and (B) Gulf of Mexico.

3.17 Observed soundings from Greensboro, NC (GSO) at (A) 12 UTC 1 March 2009 and (B) 00 UTC 2 March 2009.

3.18 Horizontal and vertical trajectories for (A) 18 UTC 1 March 2009 and (B) 06 UTC 2 March 2009 terminating at the WLZ at GSO. (C) Corresponding air parcel temperature trajectories.

3.19 Net diabatic contribution to air parcel temperature trajectories at GSO for 18 UTC 1 March 2009 (rain) and 06 UTC 2 March 2009 (snow) at 6-hr trajectory segments 
3.20 Backward ensemble trajectories for 06 UTC 2 March 2009 calculated from GSO at the near-surface level. Vertical trajectories are shown in the bottom panel

3.21 Backward ensemble temperature trajectories for 06 UTC 2 March 2009 calculated from GSO at the near-surface level.

3.22 Net diabatic contribution to air parcel temperature trajectories at GSO with near-surface temperatures (A) above freezing and (B) below freezing at 6-hr trajectory segments. Range bars indicate the maximum and minimum values among the ensemble members

3.23 Same as Figure 3.20, except at the WLZ ......................................100

3.24 Same as Figure 3.21, except at the WLZ ....................................101

3.25 Same as Figure 3.20, except at the DGZ ..................................... 102

3.26 Backward ensemble moisture trajectories for 06 UTC 2 March 2009 calculated from GSO at the DGZ with origins over the (A) Atlantic Ocean and (B) central United States.

3.27 Composite analysis of $1000 \mathrm{hPa}$ geopotential heights (meters) for all eight snowstorms at 24-hr intervals from T-72 to T-0 hours. Areas of high pressure ("H") and low pressure ("L") are noted. The position of the air parcel at each time step is denoted by a black circle. A sketch of the 72-hr composite near-surface trajectory is denoted with a solid black line and arrow at $\mathrm{T}-0$ hours

3.28 Same as Figure 3.27, except of $850 \mathrm{hPa}$ geopotential heights (meters). The position of the air parcel at each time step is denoted by a black circle. A sketch of the composite WLZ trajectory is denoted with a solid black line and arrow

3.29 Composite analysis of 1000 and $850 \mathrm{hPa}$ geopotential heights (meters) for snowstorms with DGZ-anticyclonic trajectories at T-24 hours (left column) and T- 0 hours (right column). The $850 \mathrm{hPa}$ trough axis at T-24 hours is denoted by the black dashed line. The position of the air parcel at each time step is denoted by a black circle. A sketch of the composite DGZ-anticyclonic trajectory is denoted with a solid black line and arrow....

3.30 Same as Figure 3.29, except for snowstorms with DGZ-cyclonic trajectories. The position of the air parcel at each time step is denoted by a black circle. A sketch of the composite DGZ-cyclonic trajectory is denoted with a solid black line and arrow. 
4.1 Horizontal trajectory composites of air parcels terminating at the near-surface, warm layer zone, and dendritic growth zone in the region of heavy freezing precipitation.

4.2 Vertical trajectory composites of air parcels terminating at the near-surface, warm layer zone, and dendritic growth zone in the region of heavy freezing precipitation. Composites with range bars can be found in the appendix.

4.3 Same as Figure 4.2, except for composite trajectories of air parcel temperature.....125

4.4 Same as Figure 4.2, except for moisture composites along each trajectory..........126

4.5 Same as Figure 4.2, except for composite trajectories of air parcel potential temperature...

4.6 Net diabatic contribution to air parcel temperature at each critical level in the region of heavy freezing precipitation. Range bars indicate the maximum and minimum values among all seven ice storms

4.7 Warm layer zone trajectories of observed air parcel temperature, (black line) adiabatic temperature (thick blue line), and diabatic contribution (gray bar) in the region of heavy freezing precipitation at 6-hr trajectory segments. Range bars indicate the maximum and minimum values among all seven ice storms

4.8 Horizontal and vertical trajectories for 06 UTC 5 December 2002 terminating at the near-surface level (red trajectories), WLZ (blue trajectories), and DGZ (green trajectories) at (A) Raleigh-Durham (RDU) and (B) Greensboro (GSO)

4.9 Air parcel trajectories of moisture content for 06 UTC 5 December 2002 terminating in the (A) dendritic growth zone and (B) warm layer zone at RDU and GSO....

4.10 Backward ensemble trajectories for 06 UTC 5 December 2002 calculated from RDU at the near-surface level. Vertical trajectories are shown in the bottom panel

4.11 Backward ensemble temperature trajectories for 06 UTC 5 December 2002 calculated from RDU at the near-surface level.

4.12 Backward ensemble trajectories of potential temperature for 06 UTC 5 December 2002 calculated from RDU with near-surface trajectories (A) above freezing and (B) below freezing 140

4.13 Same as Figure 4.10, except at the WLZ 
4.14 Same as Figure 4.11, except at the WLZ ....................................142

4.15 Net diabatic contribution to air parcel temperature trajectories at RDU with WLZ temperatures (A) between 5 and $8^{\circ} \mathrm{C}$ and (B) between 1 and $4^{\circ} \mathrm{C}$ at 6-hr trajectory segments. Range bars indicate the maximum and minimum values among the ensemble members. 143

4.16 Same as Figure 4.10, except at the DGZ .................................... 144

4.17 Backward ensemble trajectories of moisture content for 06 UTC 5 December 2002 calculated from RDU at the DGZ with altitudes (A) below $2 \mathrm{~km}$ and (B) above $2 \mathrm{~km}$.

4.18 Horizontal and vertical trajectories for 06 UTC 14 December 2003 terminating at the near-surface level (red trajectories), WLZ (blue trajectories), and DGZ (green trajectories) at (A) GSO and (B) RDU

4.19 Air parcel trajectories of (A) temperature and (B) potential temperature for 06 UTC 14 December 2003 terminating at the near-surface layer at RDU and GSO.

4.20 Backward ensemble trajectories for 06 UTC 14 December 2003 calculated from GSO at the near-surface level. Vertical trajectories are shown in the bottom panel.

4.21 Backward ensemble trajectories of moisture content for 06 UTC 14 December 2002 calculated from RDU with near-surface temperatures (A) above freezing and (B) below freezing

4.22 Same as Figure 4.20, except at the WLZ ....................................

4.23 Net diabatic contribution to air parcel temperature trajectories at RDU with WLZ temperatures (A) between 5 and $8^{\circ} \mathrm{C}$ and (B) between 1 and $4^{\circ} \mathrm{C}$ at 6-hr trajectory segments. Range bars indicate the maximum and minimum values among the ensemble members

4.24 Same as Figure 4.20, except at the DGZ ....................................156

4.25 Backward ensemble moisture trajectories for 06 UTC 14 December 2003 calculated from GSO at the DGZ with altitudes (A) below $2 \mathrm{~km}$ and (B) above $2 \mathrm{~km}$.....

4.26 Composite analysis of $1000 \mathrm{hPa}$ geopotential heights (meters) for all seven ice storms at 24-hr intervals from T-72 to T-0 hours. Areas of high pressure ("H") and low pressure ("L") are noted. The position of 
the air parcel at each time step is denoted by a black circle. A sketch of the composite near-surface trajectory is denoted with a solid black line and arrow

4.27 Same as Figure 3.27, except of $850 \mathrm{hPa}$ geopotential heights (meters). The position of the air parcel at each time step is denoted by a black circle. A sketch of the composite WLZ trajectory is denoted with a solid black line and arrow. The $850 \mathrm{hPa}$ trough axis at T-24 hours is denoted with a black dashed line. A composite closed low ("L") is noted at T-0 hours.

4.28 Composite analysis of 1000, 850, and $500 \mathrm{hPa}$ geopotential heights (meters) for ice storms with DGZ-Pacific trajectories at T-24 hours (left column) and T-0 hours (right column). The position of the air parcel at each time step is denoted by a black circle. A sketch of the composite DGZ-Pacfic trajectory is denoted with a solid black line and arrow. The $850 \mathrm{hPa}$ trough axis is denoted by the black dashed line

4.29 Same as Figure 4.28, except for ice storms with DGZ-Gulf trajectories. The position of the air parcel at each time step is denoted by a black circle. A sketch of the composite DGZ-Gulf trajectory is denoted with a solid black line and arrow

5.1 Daily sea-surface temperature composite for 7 January 2007 as measured from the NOAA-16 satellite. This image is qualitatively similar to the climatological East Coast sea-surface temperature pattern for the winter season. Arrows indicate the composite trajectory paths of air parcels terminating in the WLZ of heavy snowstorms (blue arrow) and ice storms (red arrow). Satellite imagery available from the Rutgers University Coastal Ocean Observation Lab: http://marine.rutgers.edu/mrs/sat_data/?product=sst\&nothumbs $=1$

5.2 Net contributions of diabatic processes to air parcel temperature at each critical vertical pressure level over the course of the 72-hr trajectory period. Positive (negative) values indicate a net diabatic warming (cooling).

5.3 A relative-wind airflow (i.e. conveyor belt) diagram based on analysis of cold season central U.S. cyclones. The solid black line with arrow is the cyclonic moist airstream of the cold conveyor belt. The dashed line with arrow is the ascending anticyclonic moist airstream of the warm conveyor belt. From Bierly (1999), his Figure 1 


\section{Chapter 1 \\ INTRODUCTION}

\subsection{Research Context}

Precipitation has long been considered one of the greatest forecasting challenges (Fritsch et al. 1998). During the winter, precipitation type (i.e. snow, sleet, freezing rain) is especially difficult to forecast because it depends on the details of the vertical temperature profile in the lower troposphere. In the Southeast U.S., precipitation-type forecasting is further complicated by the unique geography of the region, which plays an important role in the evolution of the vertical profile through complex interactions involving the synoptic-scale circulation, the regional topography (i.e. Appalachian Mountains), and nearby bodies of water (i.e. the Gulf of Mexico and Atlantic Ocean) (Figure 1.1). Cold and warm air masses often intersect across parts of the Southeast, further complicating the vertical profile. Indeed, many winter precipitation events in the region are often near a "tipping point" with regard to temperature (i.e. near $0^{\circ} \mathrm{C}$ ); therefore, subtle changes in the vertical profile can lead to significant changes in precipitation type and intensity. Moreover, some of the physical processes that affect the vertical profile are not fully represented in numerical weather prediction models (Lackmann et al. 2002), which can result in major forecast "busts" and significant societal impacts.

This dissertation project applies a synoptic perspective in the analysis of winter precipitation by explicitly linking the large-scale circulation with precipitation attributes (i.e. 
precipitation type and intensity) over a $10-y r$ period in central North Carolina. A novel aspect of this work is that the fundamental ingredients associated with winter precipitation (i.e. temperature, moisture, and lift) are linked with the large-scale circulation through the use of back trajectories. A trajectory can be defined as simply the path that an air parcel takes over space and time. With improvements in model resolution and parameterization schemes, calculating air parcel trajectories is considered a more accurate method than previously-used storm-relative isentropic analyses to envision the airflow associated with mid-latitude weather systems (Schultz 2001). Trajectory analysis is useful in determining the source regions of various meteorological parameters (e.g. temperature, moisture), tracing the evolution of their properties back across space and time, and relating changes in their properties to the physical processes that contribute to winter precipitation through identification of upstream surface and atmospheric features along each trajectory segment (Stohl 1998).

The Hybrid Single-Particle Lagrangian Integrated Trajectory (HYSPLIT) tool, developed and maintained by the National Oceanic and Atmospheric Administration's (NOAA) Air Resource Laboratory, is used in this project to calculate back trajectories associated with a sample of heavy snowstorms and ice storms that occurred within the Raleigh, NC National Weather Service (hereafter Raleigh NWS) forecast region from 2000 to 2010. Trajectory analysis represents an underutilized method and framework for analyzing the physical processes that result in heavy winter precipitation. Therefore, this research builds upon the methods and conceptual models for forecasting winter precipitation in the region that have been developed through a long-standing collaborative enterprise between the Raleigh NWS and atmospheric scientists at North Carolina State University 
(Waldstreicher 2005). Specifically, the driving hypothesis in this dissertation project is that the back trajectories identified using the HYSPLIT tool will effectively relate the changing concentration of the ingredients to the physical processes that contribute to winter precipitation, as well as to the evolving large-scale circulation regimes that assemble the ingredients across space and time.

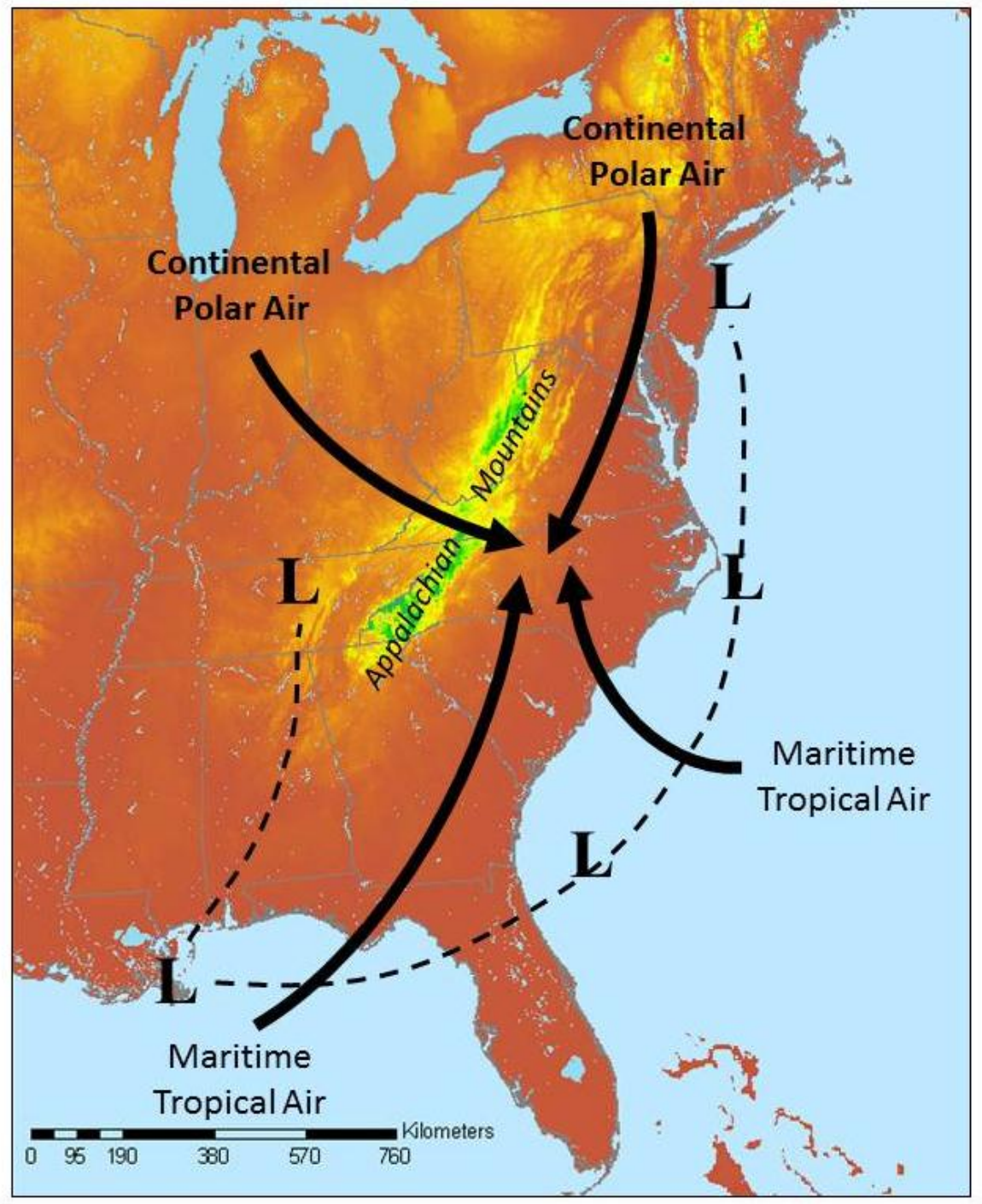

Figure 1.1. Schematic map illustrating the regional topography and the main cyclone tracks (L) and air masses connected with winter precipitation in central North Carolina (reproduced from Keeter et al. 1995). 


\subsection{Literature Synthesis}

This section discusses the various bodies of literature used to inform research on winter precipitation in central North Carolina. It begins with a description of the ingredients required for winter precipitation, followed by discussions of the atmospheric profiles and physical processes associated with different types of winter precipitation, the use of partial thickness values to determine precipitation type, and the synoptic and subsynoptic-scale features and processes associated with winter precipitation in the region. This section concludes with a discussion of the airflow characteristics (i.e. conveyor belts) associated with mid-latitude weather systems that produce winter precipitation and poses some unanswered questions regarding winter precipitation in central North Carolina.

\subsubsection{The Ingredients Required for Winter Precipitation}

Wetzel and Martin (2001) identified five key ingredients for winter precipitation: forcing for ascent (i.e. vertical motion or lift), moisture, instability, precipitation efficiency, and temperature. To illustrate how these ingredients intersect to generate precipitation, they state (p. 157-158) "some mechanism to force ascent in a region with sufficient moisture availability is required. The intensity of the ensuing precipitation can be modulated by the presence of instability and the precipitation efficiency. Finally, the precipitation type is related to the temperature profile." Thus, from this statement it can be inferred that the primary, fundamental ingredients required for winter precipitation are adequate moisture, a lifting mechanism, and a vertical temperature profile supportive of frozen (i.e. snow and sleet) and/or freezing precipitation (i.e. freezing rain). The other ingredients, instability and efficiency, may be more accurately denoted as "secondary" ingredients that are not 
fundamental or necessary to generating precipitation, but instead become important when assessing the rate (i.e. intensity) of precipitation. The concept of ingredients is useful from a forecasting perspective because it allows the forecaster to focus only on the most relevant aspects of a weather event, making the task of analyzing and interpreting model output and diagnostic products more manageable (Schultz et al. 2002).

\subsubsection{Winter Precipitation Types}

\subsection{2a. Definitions and Distributions}

The three primary winter precipitation types are freezing rain, sleet, and snow. Freezing drizzle occurs much less frequently in the Southeast than in other regions and is not examined in detail here. Nearly all winter precipitation events begin with ice crystals and snowflakes forming in cold clouds (cloud-top temperatures $<-10^{\circ} \mathrm{C}$ ) via deposition and growing through aggregation and riming. As they fall from the cloud, they either melt completely (i.e. rain or freezing rain), partially (i.e. sleet), or remain frozen (i.e. snow). In the case of freezing rain and sleet, an elevated warm (i.e. melting) layer exists above a surface-based dome of subfreezing air (Figure 1.2a and b).

Whether an ice crystal or snowflake melts, either partially or completely, depends on the properties of the warm layer (i.e. temperature, depth, lapse rate, humidity), the size, density, and structure of the crystal or snowflake, and the fall velocity (Czys et al. 1996; Zerr 1997; Wandishin et al. 2005). The presence or absence of a warm layer aloft is typically determined by checking the vertical temperature profile from a radiosonde launch, or sounding, but may also be determined remotely using satellite data and radar signatures. For example, Martner et al. (1993) studied the radar characteristics of a severe Midwest ice storm 
and found that locations of freezing rain were collocated with regions of enhanced radar reflectivity, termed a "bright band". They concluded that the bright band signature was caused by the presence of liquid drops or partially-melted ice crystals aloft, which have a higher base reflectivity than solid hydrometeors. Similarly, the bright band radar signature has been observed in regions of heavy wet snow and sleet, where liquid water is present around solid hydrometeors.

In the case of freezing rain, a sufficiently warm and deep melting layer is required for snow and ice to melt completely (Figure 1.2a). Bernstein (2000) found that maximum warm layer temperatures associated with freezing rain were at least $4^{\circ} \mathrm{C}$ at Greensboro, $\mathrm{NC}$ (GSO). A shallow dome of cold air is also required so melted raindrops do not re-freeze before contacting the ground or a suspended object. In the case of sleet, the melting layer is cooler and thinner such that snow and ice do not completely melt. Upon reaching the low-level cold dome these partially melted hydrometeors re-freeze into ice pellets when the minimum temperature in the cold dome is less than $-7^{\circ} \mathrm{C}$ (Bernstein 2000). Although snow most typically reaches the ground in situations where the entire atmospheric column is below freezing (i.e. snowflakes do not melt and re-freeze, Figure 1.2c), laboratory experiments have shown than falling snow may not melt completely if the air temperature is above $0^{\circ} \mathrm{C}$ and unsaturated (Matsuo and Sayo 1981). In these cases, the cooling effect from evaporation exceeds the warming effect from conduction and the surface of the crystal or snowflake remains subfreezing. There are also situations where diabatic cooling due to melting and sublimation erode a surface-based warm layer and lead to a transition from rain to snow (Figure 1.2d). 
The spatial distribution of winter precipitation types across North Carolina is influenced primarily by elevation and proximity to the Atlantic Ocean. Snow is most common in the mountains where temperatures are more likely to be subfreezing through the atmospheric column, while marine influences and warmer soils typically hinder the occurrence of winter precipitation and accumulation along the coast. In between these regions is an area called the "transition zone", which broadly encompasses the Piedmont region of central North Carolina and extends into central Virginia, South Carolina, and parts of north Georgia (Keeter et al. 1993; Keeter et al. 1995; Gurka et al. 1995). In some cases, the transition zone is simply the boundary between frozen and liquid precipitation (i.e. the "rain-snow line"). In many other cases, however, the actual transition zone is not a discrete boundary, but instead is an area of varying width consisting of various combinations of precipitation types (i.e. "mixed" precipitation). Keeter et al. (1993) studied a sample of winter precipitation events in North Carolina from 1960 to 1990 and classified them as "narrow" or "broad" according to the width of their transition zone. Narrow transition zones were 30 to $75 \mathrm{~km}$ wide and remained relatively stationary over the duration of the event (Figure 1.3a). Events with a narrow transition zone were typically associated with strong thermal advection and strong dynamics (i.e. enhanced lift) connected with a mature surface cyclone and upper-level divergence. Broad transition zones ( $>75 \mathrm{~km}$ wide) were also associated with strong thermal advection, but relatively weak dynamics (Figure 1.3b). In these cases, the type of precipitation observed in the transition zone was influenced largely by diabatic processes resulting from energy exchanges between precipitation and the surrounding air (e.g. cooling associated with melting snowflakes). A discussion of the physical processes that affect precipitation type follows. 


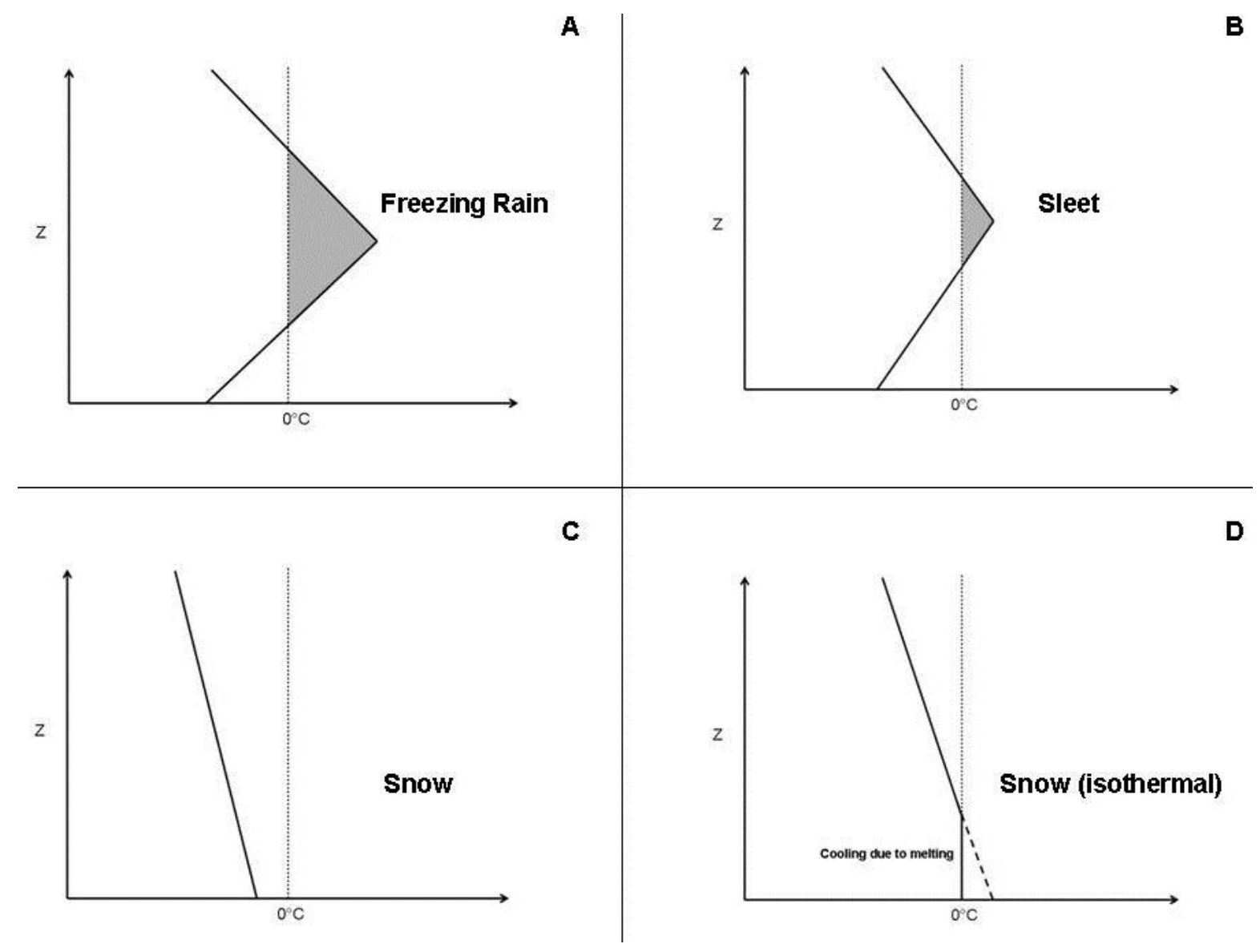

Figure 1.2. Schematic showing the vertical thermal profiles associated with (A) freezing rain, (B) sleet, (C) snow, and (D) snow with an isothermal layer resulting from diabatic cooling. The dotted vertical line represents the $0^{\circ} \mathrm{C}$ isotherm and the thick line represents the environmental lapse rate. 

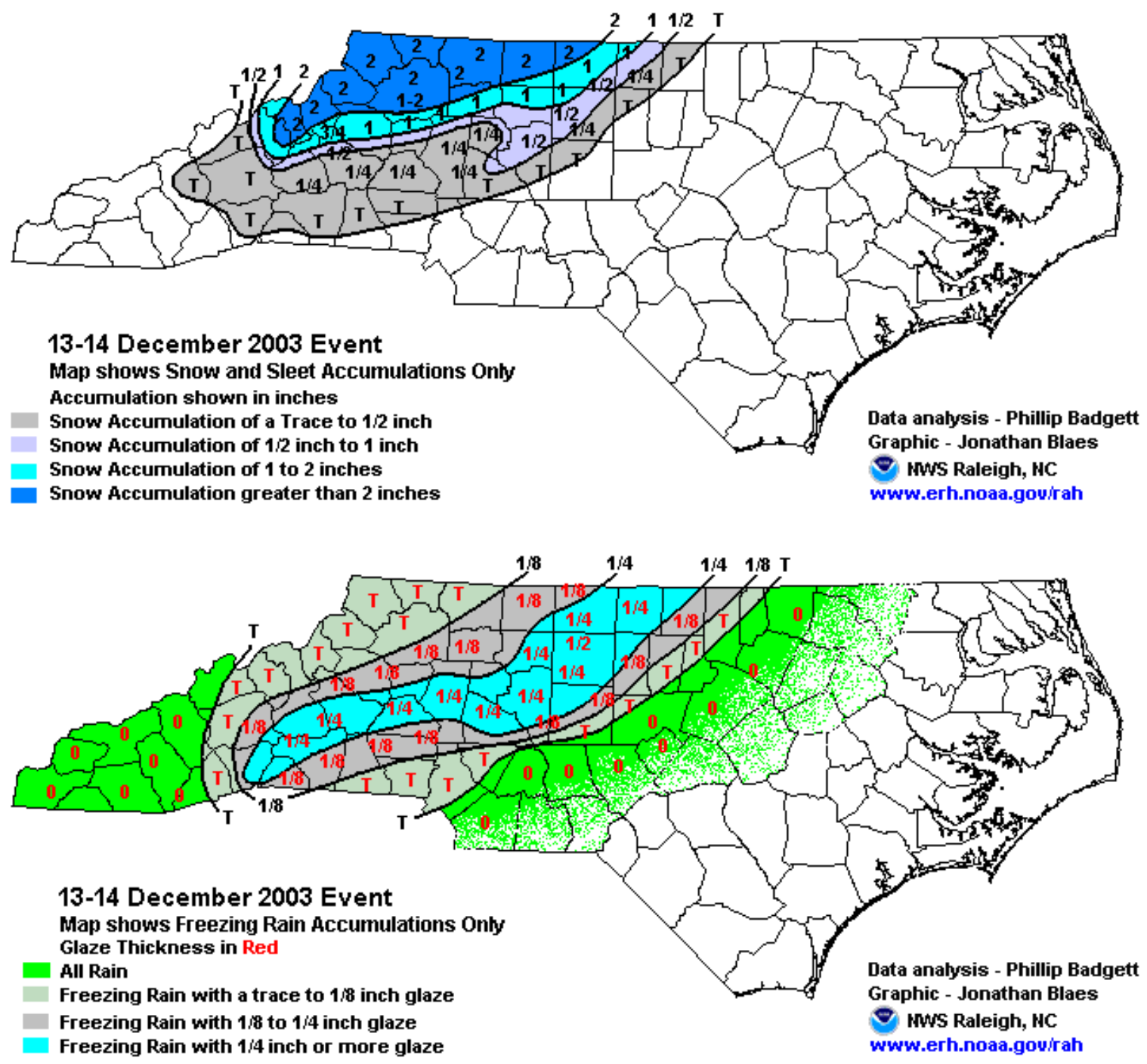

(A)

Figure 1.3. Examples of winter storms in North Carolina associated with (A) a narrow transition zone of freezing rain (bottom image) juxtaposed with a region of snow to the northwest (top image) and (B) a broad transition zone of mixed precipitation across most of central North Carolina. Figures produced by the Raleigh NWS forecast office. 


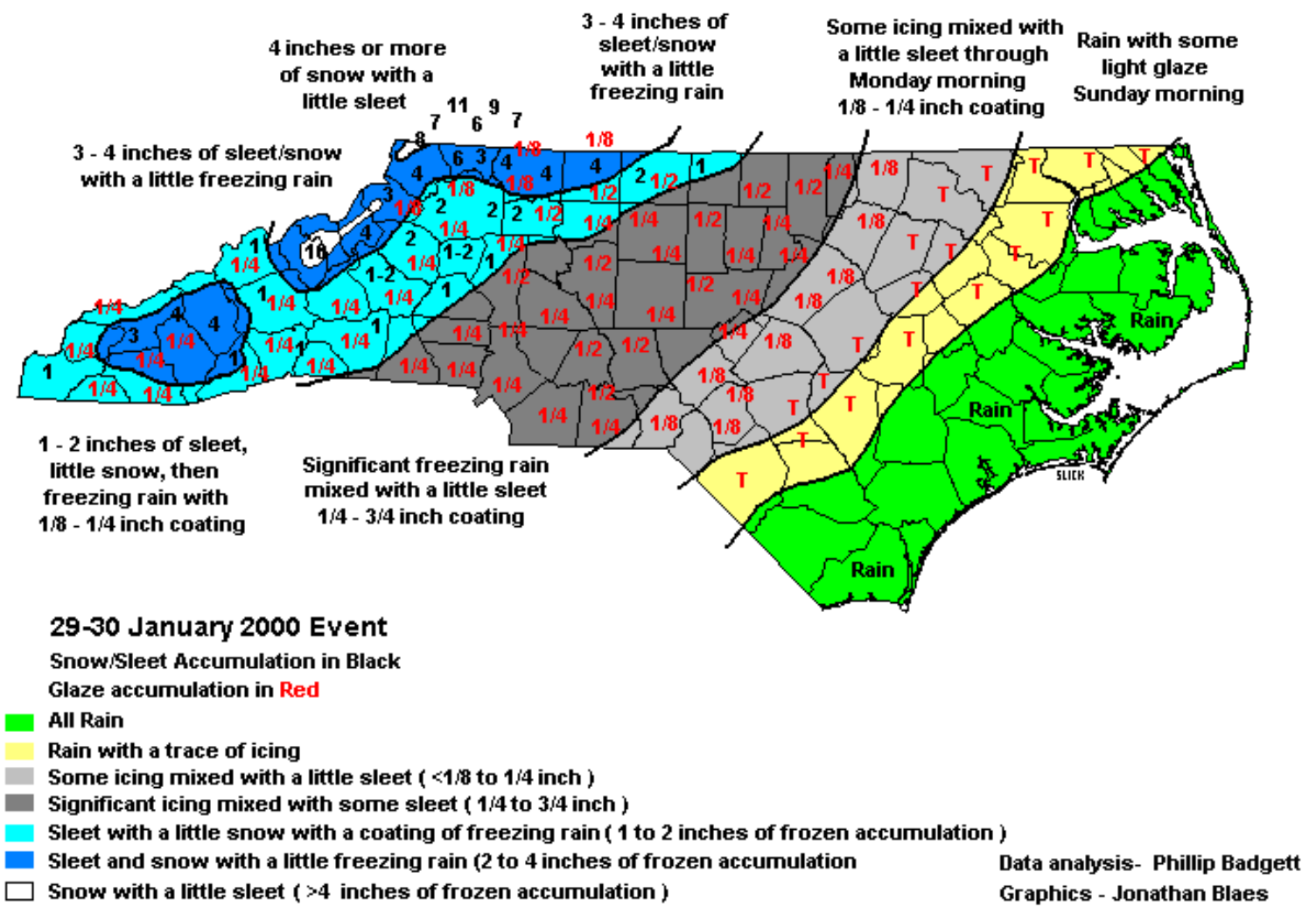

(B)

Figure 1.3. Continued 


\subsection{2b. Physical Processes Affecting Precipitation Type}

The vertical temperature profile associated with winter precipitation is not static, but evolves throughout the course of an event due to a combination of dynamic and thermodynamic processes. To a first approximation, a change in temperature at a given point results from thermal advection, vertical motion (i.e. adiabatic warming or cooling), and/or diabatic processes (e.g. melting, freezing, sublimation, solar heating) (Holton 2004). Assessing the relative importance and strength of each of these terms is a major forecast challenge and is often the key to determining precipitation type.

In some cases, large-scale thermal advection (e.g. cold-air advection from an anticyclone anchored northeast of the region) and vertical motion are the primary factors in determining the distribution of precipitation types, while in other cases the local effects of melting, sublimation, and freezing can be of equal or greater importance. These local effects result from the release (absorption) of latent heat caused by the freezing (evaporation and melting) of precipitation. Additionally, the release of latent heat due to condensation and deposition results in local warming near the region of precipitation formation. Although the latent heat associated with melting and freezing is small relative to that from evaporation and condensation, it is often concentrated within shallow atmospheric layers and can, therefore, produce locally significant alterations to the temperature profile (Lackmann et al. 2002). For instance, in the absence of a near-surface cooling mechanism, the heat released from freezing rain can warm up and potentially eradicate the surface-based subfreezing layer, making freezing rain a self-limiting process (Stewart 1985). This is particularly the case with heavy freezing rain, which would help remove the subfreezing layer sooner because an increase in 
the rate of freezing would increase the upward transport of latent heat from the surface (Lackmann et al. 2002).

Similarly, the cooling effect of melting precipitation is directly proportional to the precipitation rate. This relationship between precipitation rate and the release or absorption of latent heat affects a critical aspect of winter weather forecasting: the quantitative precipitation forecast (QPF). Many numerical weather forecast models are not configured to adequately account for these processes in situations linked to QPF. Therefore, substantial temperature biases can arise because of the manner in which some models (particularly the National Center for Environmental Protection's Eta Model) represent phase changes of precipitation. Lackmann et al. (2002) showed that the inability of the Eta Land-Surface Model to resolve the latent heat of freezing and upward heat flux during a winter storm in central North Carolina resulted in an overestimation and subsequent over-forecasting of freezing rain.

Several other studies have assessed the impact of diabatic processes on the vertical temperature profile (and resulting precipitation type) during winter precipitation events. For example, Kain et al. (2000) examined a major snow event in eastern Tennessee that began as heavy rain and quickly transitioned to heavy, wet snow. Over 10 in $(25.4 \mathrm{~cm})$ of snow was reported near Knoxville, TN following a period of heavy rain with temperatures several degrees above freezing. A version of the National Center for Atmospheric Research-Penn State University mesoscale model MM5 was able to resolve the persistent cooling effect of melting snow in a deep, surface-based warm layer. Over time, the cooling effect of melting snowflakes allowed the $0^{\circ} \mathrm{C}$ isotherm to progress downward through the troposphere, eventually reaching a level near the surface where snowflakes could reach the ground. In this 
case, cooling due to melting was the dominant factor in determining precipitation type; vertical motions and horizontal temperature advections were weak in the vicinity of the rainto-snow changeover. Moreover, the high rate of cooling was associated with the intensity of the precipitation; lighter precipitation may not have cooled the atmosphere sufficiently to support snow down to the surface in the absence of other processes. A rule-of-thumb checklist was presented to aid forecasters in assessing the role of cooling due to melting in similar synoptic situations.

In another case, the effect of latent heating on the warmth and depth of the elevated warm layer was assessed by Ramos da Silva et al. (2006) using the Regional Atmospheric Modeling System. They found that warming due to latent heat release in the marine atmospheric boundary layer associated with anomalously warm Atlantic sea-surface temperatures (SSTs) led to greater melting layer depths and more freezing rain in parts of North Carolina and Virginia. When ocean temperatures were cooler, snow was more likely to occur. It was also found that the effect of Atlantic SSTs was confined to elevated warm layers; surface-based cold air masses were not affected by changes in Atlantic SSTs using the regional model.

\subsection{2c. Using Partial Thickness to Forecast Precipitation Type}

Thicknesses between different pressure levels in the atmosphere are of great importance in determining precipitation type (Heppner 1992). This is because the distance (i.e. thickness) between two levels is positively correlated with the mean temperature of the air column between them. Wagner (1957) determined that a 1000-500 hPa thickness of 5400 $\mathrm{m}$ best demarcated the boundary between frozen and liquid precipitation, except in regions of 
high elevation. This technique is still used routinely as a "first-cut" to determine the location of the rain-snow line.

However, to determine the boundaries and locations of frozen, liquid, and freezing precipitation (i.e. the transition zone), it is useful to further divide the lower-troposphere into two main layers: 1000-850 hPa, where the surface-based cold dome usually resides, and 850$700 \mathrm{hPa}$ where an elevated warm layer is commonly present (Gay and Davis 1993). Critical thicknesses of these layers are combined to produce regression equations that give the probabilities of an observed precipitation type (Bocchieri 1980; Keeter and Cline 1991). The greatest probabilities are depicted visually on a partial thickness nomogram, which can be used to differentiate between various precipitation types (Figure 1.4). The nomogram shows the distribution of precipitation types as a function of partial thickness values. Mid-level (i.e. 850-700 $\mathrm{hPa}$ ) thickness values increase from left to right along the horizontal axis in Figure 1.4, while low-level (i.e. $1000-850 \mathrm{hPa}$ ) thickness values increase from bottom to top along the vertical axis. The critical thicknesses associated with each precipitation category in the nomogram are generally applicable for locations less than $450 \mathrm{~m}(1500 \mathrm{ft})$ in elevation, excluding the coastal plain. While useful, the nomogram does have important limitations. Namely, cloud microphysics, diabatic processes, and surface conditions are not directly accounted for in the nomogram. It also does not include trace precipitation events and does not resolve freezing rain events that occur with an exceptionally shallow cold dome (i.e. below $950 \mathrm{hPa})$.

The first version of the nomogram was developed in the early 1990s by the Raleigh NWS using radiosonde data and precipitation observations from winter storms collected over a 30-yr period at GSO. Figure 1.4 shows the most recent version, which was last updated in 
2007 with refinements to the middle portion of the nomogram where diabatic processes have a significant effect on precipitation type. Cuviello (2007) refined the nomogram using data from GSO for the period 1957-1995. His nomogram correctly classified 62 percent of the precipitation types, an improvement over the original nomogram.

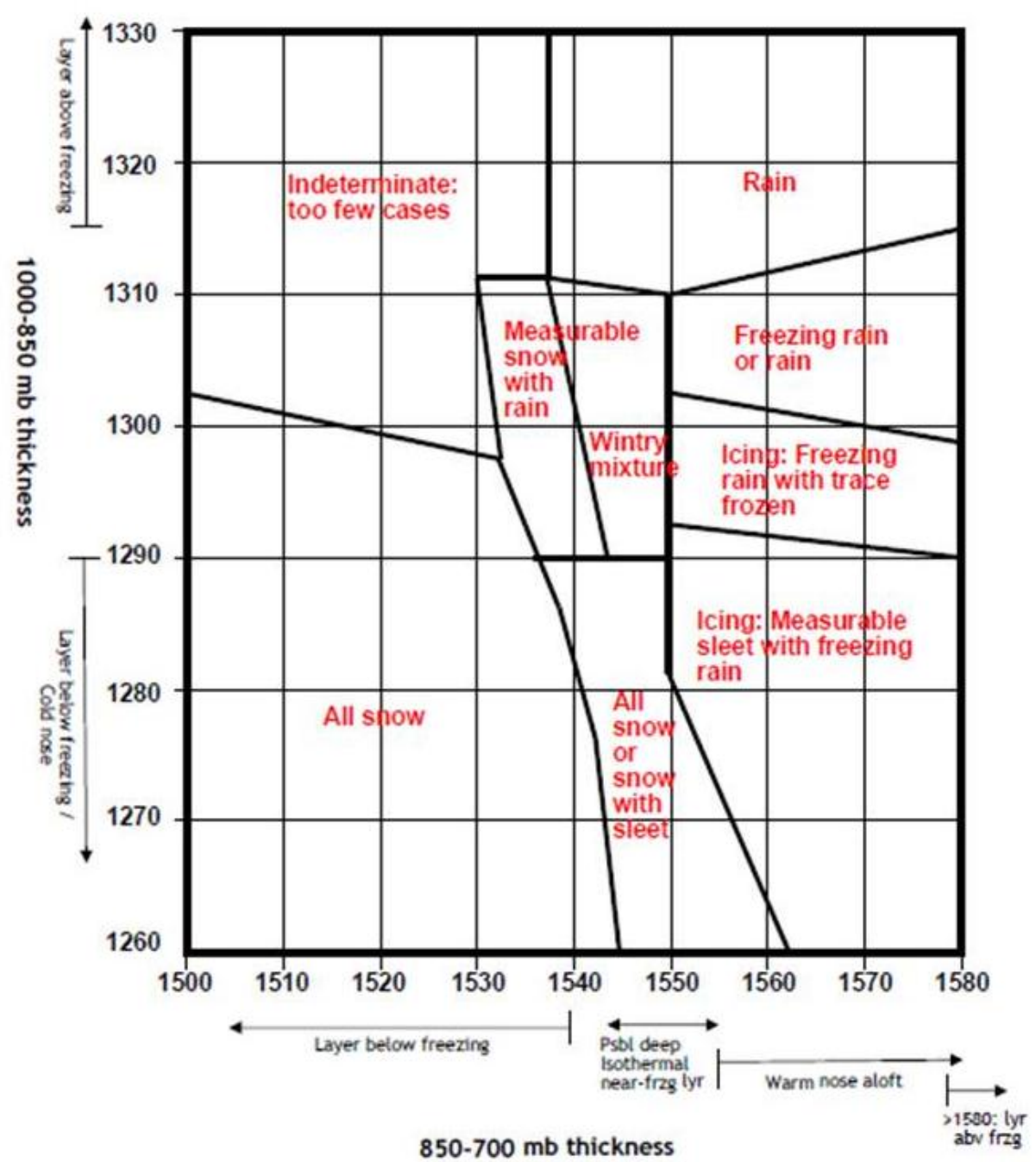

Figure 1.4. Partial thickness nomogram for Greensboro, NC (GSO) produced by the Raleigh NWS forecast office. Thickness values given in meters. 


\subsubsection{Synoptic-Scale Features Associated with Winter Precipitation}

Winter precipitation in central North Carolina can be tied to various synoptic-scale features, which include surface features such as cyclones and anticyclones (and their associated frontal boundaries) as well as upper-level features, such as jet streams and jet streaks. These features play important roles in assembling the ingredients necessary to generate winter precipitation (i.e. temperature, moisture, lift).

Nearly three-quarters of all winter storms that affect central North Carolina are associated with a surface wave or mid-latitude cyclone (Cuviello 2007). Cyclones bring together a wide range of processes in a strongly baroclinic environment (i.e. one in which there is the juxtapositioning of cold and warm air), including entrainment of water vapor and enhanced lift (Kocin and Uccellini 2004). Cyclonic systems that are responsible for many of the major winter storms in central North Carolina generally take a "Gulf-Atlantic" track (i.e. Miller-type A, Miller 1946; Figure 1.5). This type of cyclone produces many heavy snowfall events accompanied by relatively narrow transition zones (Keeter et al. 1993; Mote et al. 1997). The "Atlantic Coast Redevelopment" cyclone track (i.e. Miller-type B, Figure 1.6) is often tied to heavy freezing rain events, a broad transition zone, and warm-air advection in an elevated warm layer (Keeter et al. 1993; Cuviello 2007). These events generally exhibit weak intensification of the primary cyclone as it moves inland, while the secondary coastal cyclone deepens off of the East Coast.

The cold air necessary for winter precipitation is typically supplied to the region via a surface anticyclone anchored over the Northeast U.S. or southeast Canada (Mote et al. 1997; Bernstein 2000; Robbins and Cortinas 2002). Most heavy freezing rain and sleet events in the Southeast are associated with strong anticyclones $(1029-1044 \mathrm{hPa})$ that advect a 
replenishing supply of cold dry air into the region (Cuviello 2007). This process can compensate for the diabatic heating associated with the freezing of rain droplets, increasing the potential for significant ice accumulation. The trajectory of this air is strongly influenced by the Appalachian Mountains, which can block its westward progression allowing cold, dense air to pool in a shallow atmospheric layer across central North Carolina. This phenomenon is referred to as cold-air damming and will be discussed in the next subsection. In contrast, the majority of heavy snowfall events (58 percent) are associated with a weaker anticyclone (1014-1031 hPa) (Cuviello 2007). In many of these cases, the near-surface air is not as cold; therefore heavy snow development requires other processes, such as cold-air advection in the western quadrant of a strong cyclone and diabatic cooling due to sublimation and melting of snowflakes in the lower troposphere.

Winter precipitation in central North Carolina can be generated through a variety of lifting mechanisms. The most important mechanisms include upper-level divergence and frontal lifting. Upper-level divergence occurs when air speeds up aloft, forcing air below to rise and cool adiabatically. This leads to condensation and deposition and, if enough moisture is available, the development of precipitation. Divergence aloft typically occurs downstream of an upper-level tough and can be enhanced in regions where wind speeds are particularly fast (i.e. right rear or left forward quadrant of a jet streak). Upper-level troughs are also associated with areas of cyclonic shear and spin (i.e. vorticity), which when advected downstream can lead to locally enhanced regions of upper-level divergence.

Frontal lifting occurs when two air masses of different thermodynamic characteristics converge, forcing air to rise. In central North Carolina, winter precipitation connected with frontal lifting typically occurs when warm, moist surface air rises over a dome of cold, dry 
surface air. This is referred to as isentropic lifting because the advection of warm, moist air into the region usually follows lines of equal potential temperature, or isentropes. Mote et al. (1997) determined that isentropic lifting associated with an intensifying low-level jet was the dominant lifting mechanism during most heavy snowstorms in the Southeast U.S., with jet streak dynamics and upper-level divergence termed "secondary" mechanisms. The low-level jet is also a key feature in supplying moisture for precipitation growth and decreasing the low to mid-level stability via warm-air advection.

Atmospheric stability describes the propensity for air parcels to rise or fall due to differences in buoyancy (e.g. warm, moist air situated below cold, dry air). When the temperature lapse rate (i.e. change in temperature with height) is steep the atmosphere is termed "unstable" and air parcels tend to rise convectively. The atmospheric stability decreases when surface air warms via solar insolation or low-level warm-air advection. Stability is further reduced when the air aloft is especially cold; various processes provide cooling, including cold-air advection, adiabatic cooling in regions of upper-level divergence, and diabatic cooling due to evaporation and sublimation from the tops of mid-level clouds. Decreases in atmospheric stability can result in areas of enhanced lift and precipitation formation. Additionally, sensible and latent heat fluxes from the Atlantic Ocean can strengthen the low-level jet (i.e. warm-air advection) by increasing the thermal and pressure gradient between air over the land and air over the water, thereby strengthening the wind field and increasing the water vapor content in the jet (Kocin and Uccellini 2004).

Though frontal lifting often occurs in association with a surface cyclone, isentropic lift can occur in the absence of a surface cyclone, as was the case during the record-setting snowfall experienced in northwestern South Carolina in January 1988 (Moyer 2001). The 
features that promoted strong isentropic lift and heavy snow in that case were a cut-off low pressure at $850 \mathrm{hPa}$ (i.e. strong warm-air advection due to southerly low-level flow) and jet streaks moving through a fast upper-level jet stream (i.e. increased cyclonic vorticity and decreased mid-level stability). The combination of cyclonic vorticity and warm-air advection often results in greater lift over the region of winter precipitation (Mote et al. 1997; Robbins and Cortinas 2002). 


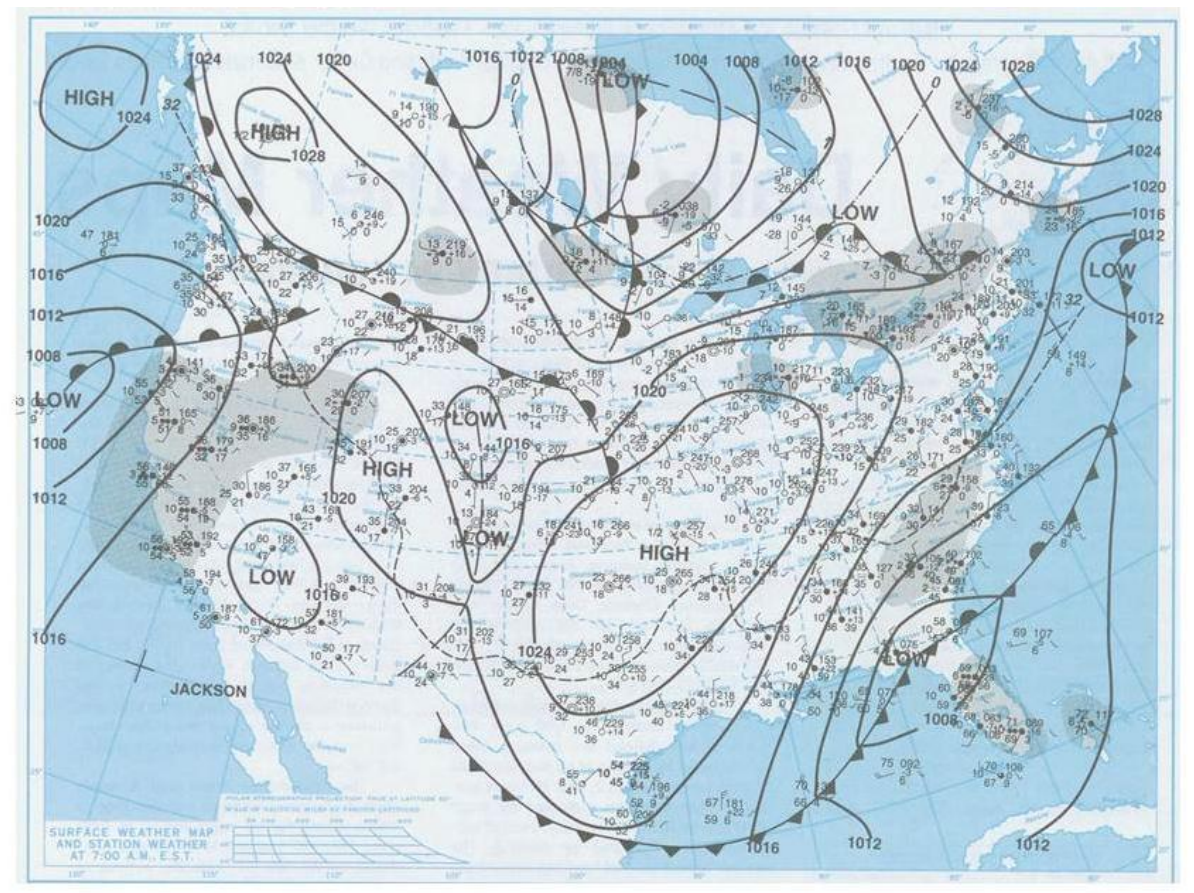

(A)

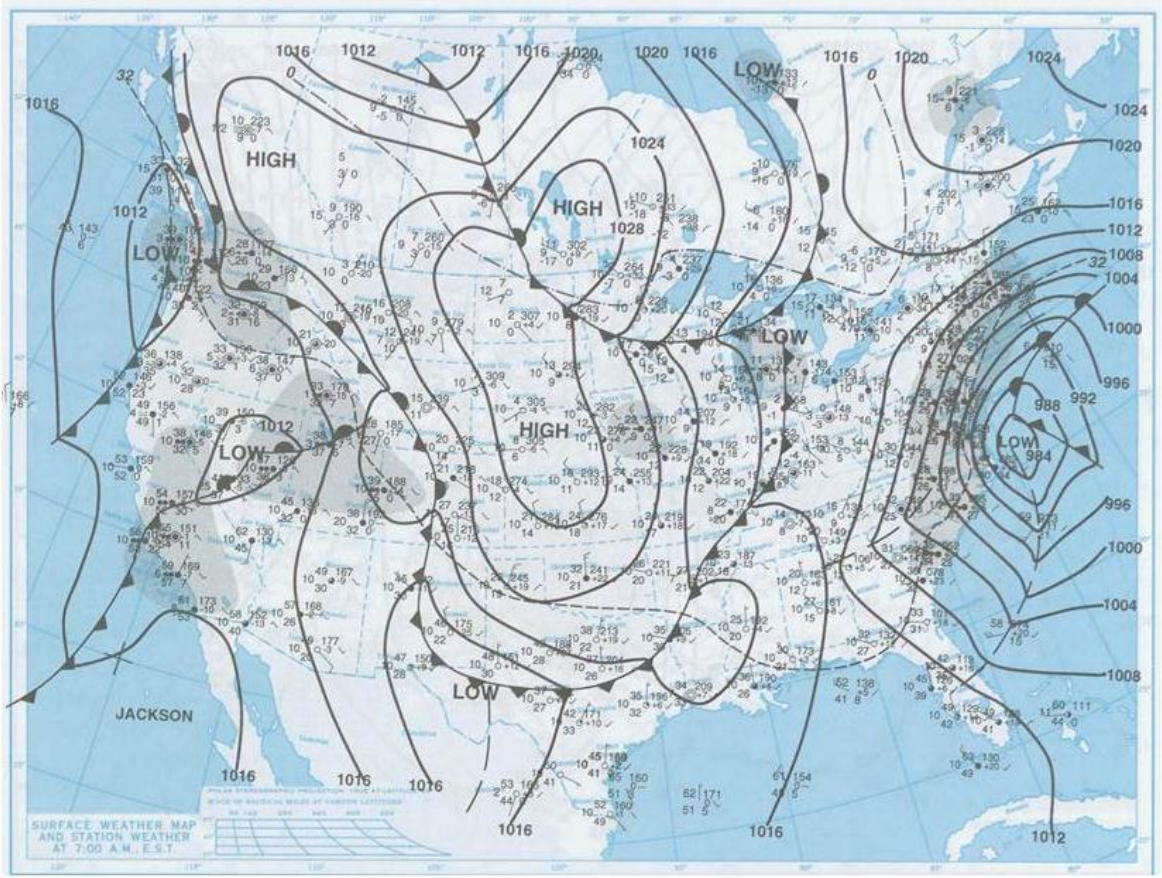

(B)

Figure 1.5. Example of a "Gulf Atlantic" or Miller-A cyclone type at (A) 12 UTC 24 January 2000 and (B) 24 hours later (12 UTC 25 January 2002). 


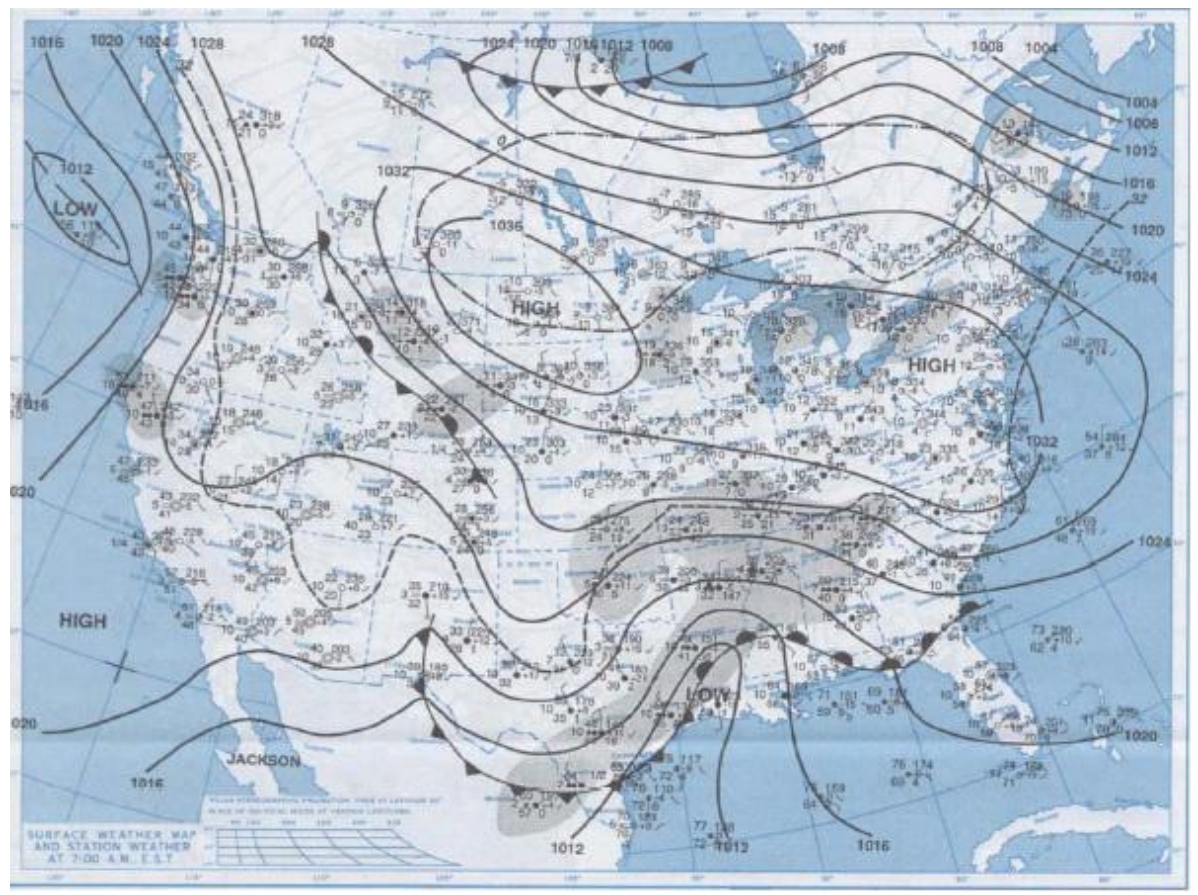

(A)

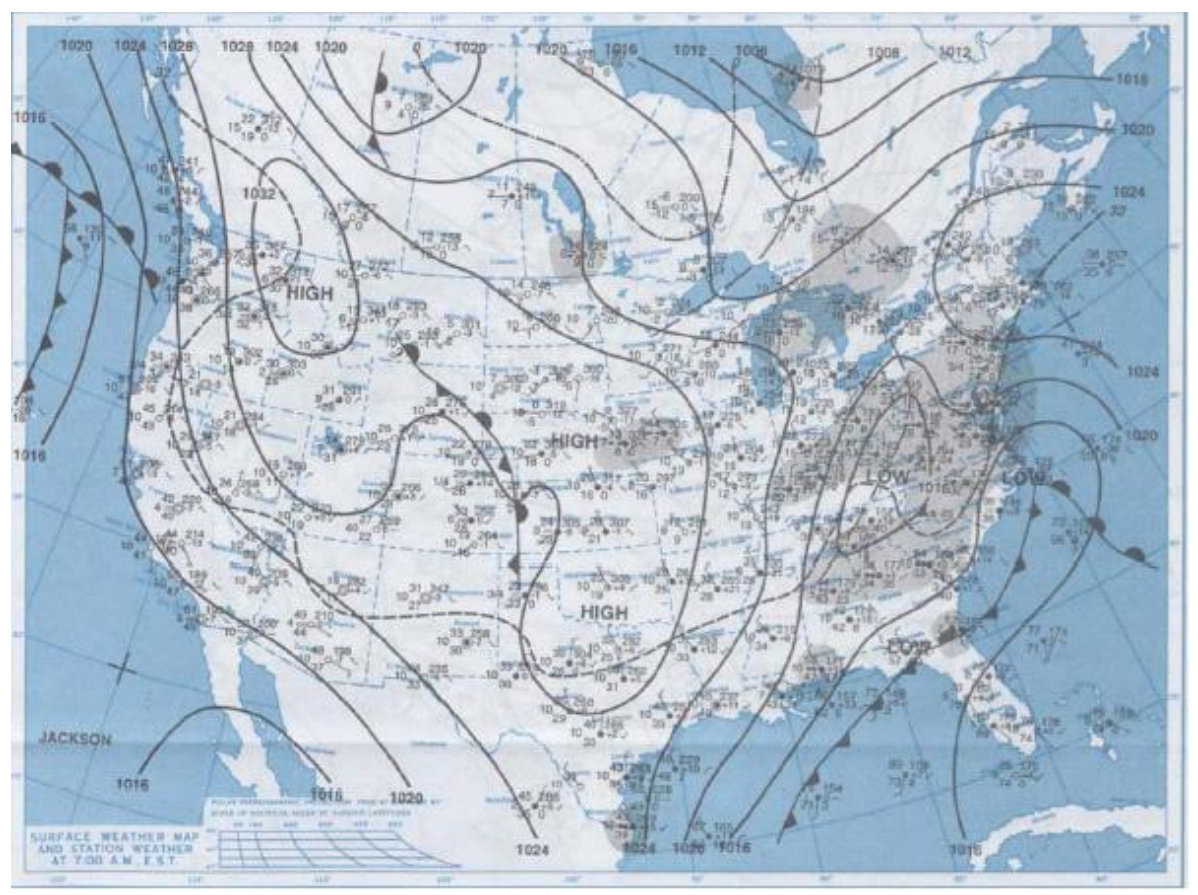

(B)

Figure 1.6. Example of an "Atlantic Coast Redevelopment" or Miller-B cyclone type at (A) 12 UTC 4 December 2002 and (B) 24 hours later (12 UTC 5 December 2002). 


\subsubsection{Subsynoptic-Scale Features and Processes Associated with Winter Precipitation}

\subsection{4a. Cold-Air Damming}

Many winter precipitation events in central North Carolina are associated with a phenomenon called cold-air damming (CAD). This phenomenon is characterized by a $\mathrm{U}$ shaped pattern in the surface isobars along the east slopes of the Appalachian Mountains and Piedmont, extending from the mid-Atlantic region to as far south as northern Georgia (Forbes et al. 1987) (Figure 1.7). Observance of this pressure pattern coincides with a surface anticyclone centered over the Northeast U.S. or southeast Canada. Richwien (1980) used the term "damming" to refer to the effect of the mountain barrier on the horizontal flow of air. While CAD occurs most frequently during the warm season, its greatest impact on sensible weather (i.e. cold temperatures and winter precipitation) occurs during the winter season (Bailey et al. 2003). CAD events can also influence cyclogenesis and the cyclone track along the East Coast by increasing the thermal gradient between the cold land mass and the warm Gulf Stream waters. The resulting low-level baroclinic zone enhances surface convergence, leading to increased lift, stronger moisture advection, and heavier precipitation (Kocin and Uccellini 2004; Appel et al. 2005).

Since CAD often signals the presence of a surface-based air mass conducive to winter precipitation, it is important for forecasters to be able to determine the processes that maintain and erode it. Maintenance of the CAD has been tied to the anchoring of a strong anticyclone to the northeast, resulting from upper-level confluence and sinking air, as well as diabatic cooling due to evaporation and sublimation from shallow clouds above the cold dome (Bailey et al 2003). Erosion of CAD, which typically signals a transition from winter precipitation to liquid as the surface-based layer warms, has been tied to a several different 
synoptic patterns (Lackmann and Stanton 2004). These include the development of a coastal cyclone, resulting in cold-air advection aloft and weakening of the low-level inversion above the cold dome; the movement of the anticyclone offshore, resulting in the elimination of the cold dome due to solar heating; and the passage of a cold front associated with a surface cyclone located to the northwest. This particular setting is often associated with the inland progression of a coastal front, which is generally not well-depicted by numerical weather prediction models (Appel et al. 2005).

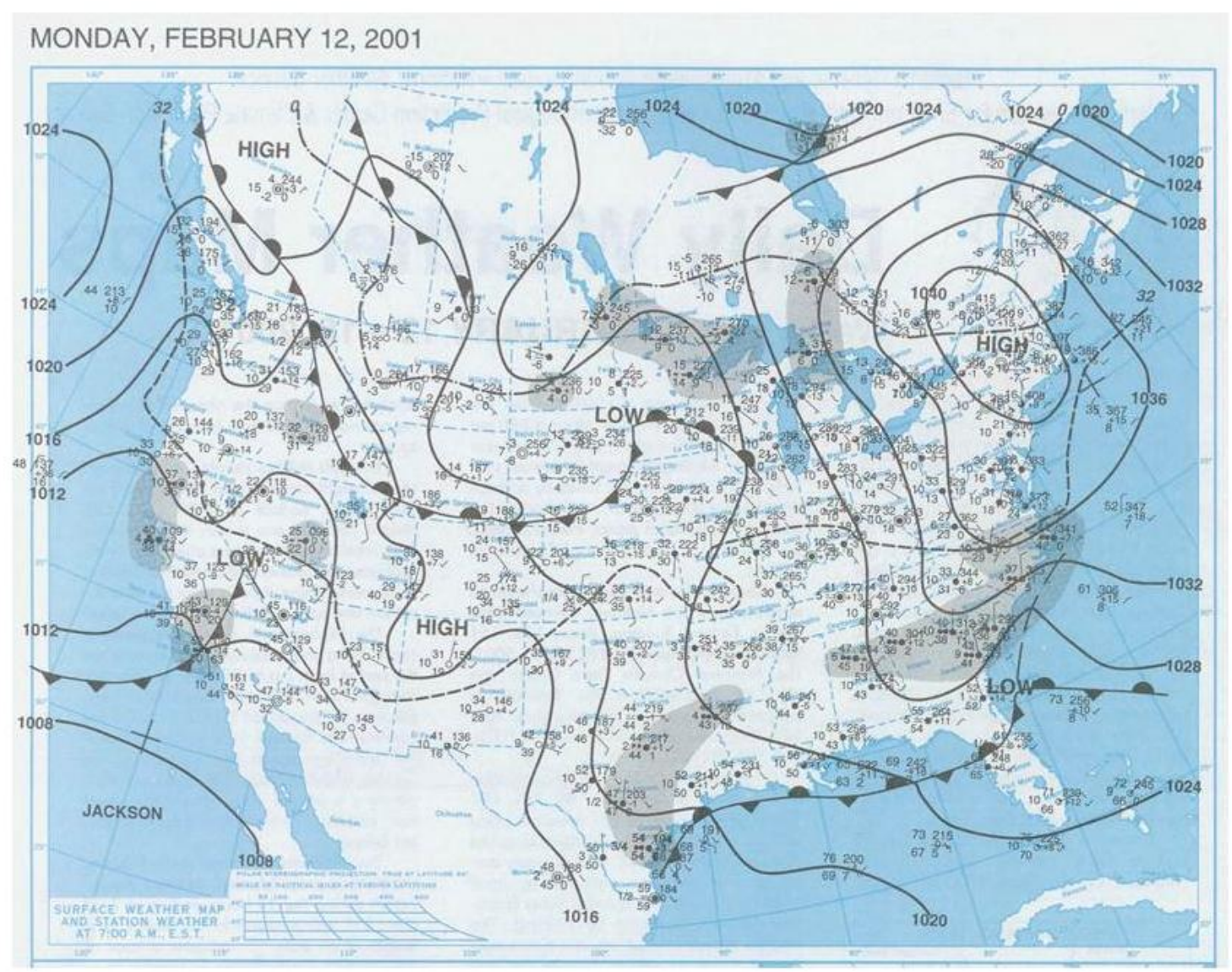

Figure 1.7. Surface weather map showing a well-defined cold-air damming (CAD) signature along the U.S. East Coast. 


\subsection{4b. Upstream Convection and Instability}

In cases of strong isentropic lift along the Gulf Coast, where a stationary front separates the subfreezing land mass from the warm waters of the Gulf of Mexico, convective storms with frequent cloud-to-ground lightning are sometimes observed on the south (i.e. warm) side of the frontal boundary (Hunter et al. 2001). It has been hypothesized that ice crystals from these convective clouds may be advected northward over the frontal boundary and "seed" low-level clouds above the downwind subfreezing air mass (Figure 1.8; Hunter et al. 2001). The induction of ice crystals into relatively shallow, supercooled clouds can result in higher rates of deposition and enhanced precipitation. In cases where upstream convection is oriented north-to-south (e.g. ahead of a cold front), downstream precipitation can be either enhanced or inhibited depending on the speed of the convective system as it progresses eastward (Mahoney and Lackmann 2007). Slower-moving systems may strengthen the northward progression of the low-level jet, enhancing downstream precipitation. Fastmoving systems, on the other hand, disrupt the low-level jet due to moisture consumption and latent heat release (i.e. subsidence and drying of the downstream environment). 

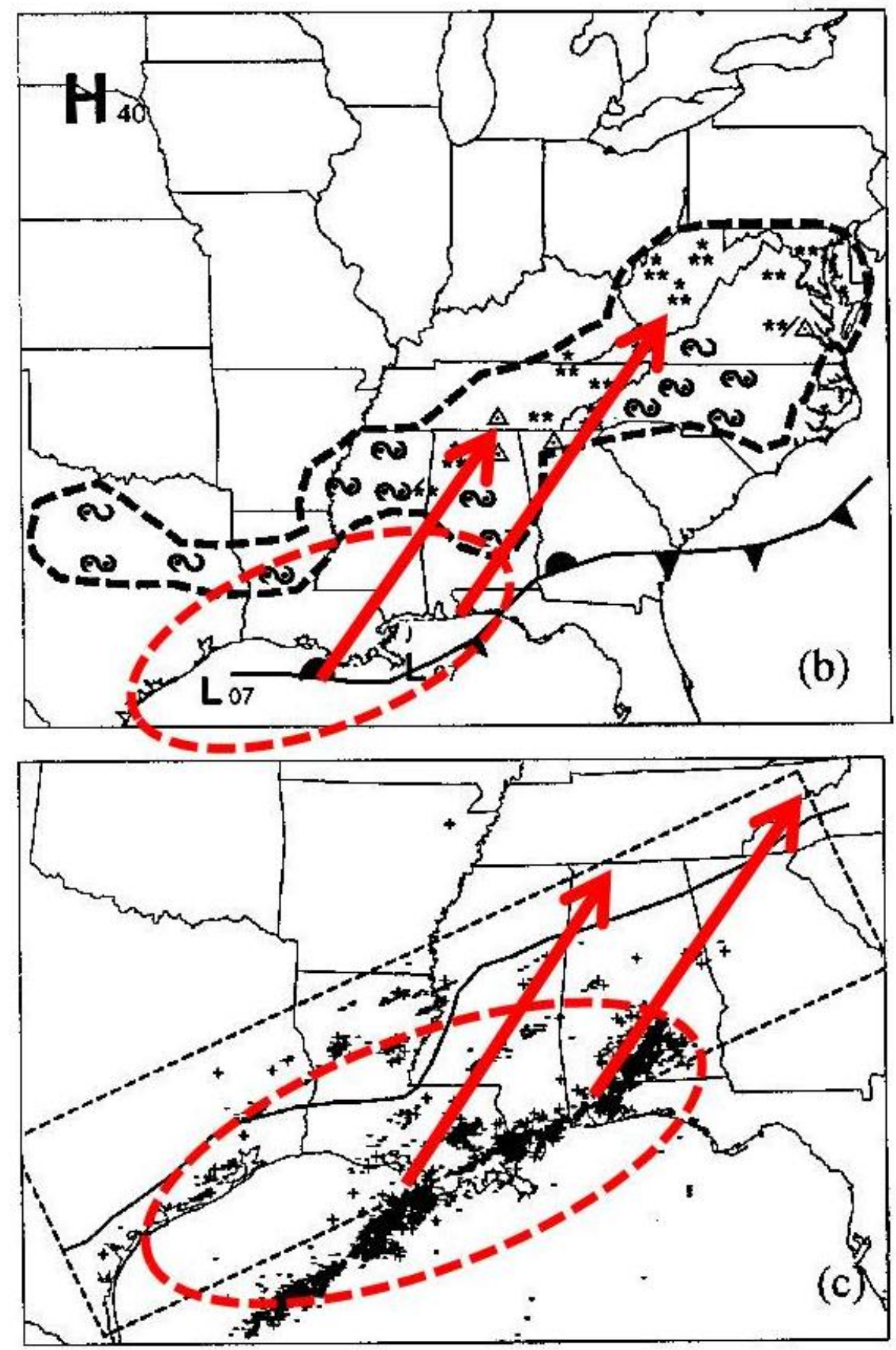

Figure 1.8. Surface analysis of frontal boundaries and precipitation during a severe ice storm (top) and corresponding pattern of cloud-to-ground lightning flashes (bottom). The general region of convection is highlighted in both images by red ovals. The mean steering flow is indicated with red arrows. This suggests that ice from the tops of the convective clouds were advected downwind, helping to enhance precipitation rates north of the frontal boundary. From Hunter et al. (2001), their Figure 3. Red ovals and arrows added for illustration. 


\subsubsection{The Conveyor Belt Model}

The study of airflow associated with extra-tropical (e.g. mid-latitude) cyclones has evolved significantly since the original two-dimensional cyclone model put forth by the Bergen School in the early $20^{\text {th }}$ century (Bjerknes 1919). The first three-dimensional “conveyor belt" models were developed by Danielson (1964), Browning et al. (1973) and Harrold (1973) and were later refined by Carlson (1980), Carr and Millard (1985), and Browning (1990). A conveyor belt can be envisioned as an ensemble of air parcels, originating from a common source region, tracked over synoptic-scale time periods (24-48 hours) (Moore et al. 2005). In a mature cyclone, each conveyor belt can be as much as $2 \mathrm{~km}$ in depth and several hundred kilometers wide (Eckhardt et al. 2004; Ng 2005). From a trajectory perspective, conveyor belts are best represented by collections of trajectories that follow similar paths, though not all of the airflow in a cyclone will coincide with these aggregates of trajectories (Schultz 2001). Nevertheless, the configuration and characteristics of conveyor belts and their boundaries are important to the development of extra-tropical cyclones and the production of clouds and precipitation, and have therefore become an important conceptual framework for weather forecasting (Bierly and Winkler 2001).

To date, the most widely accepted model of conveyor belts associated with extratropical cyclones is that of Carlson (1980), who identified three primary airstreams: the dry conveyor belt (DCB), the warm conveyor belt (WCB), and the cold conveyor belt (CCB) (Figure 1.9). The DCB originates near the tropopause and descends along the western branch of an upper-level trough. The air descends due to confluence in the right entrance region of an upper-level jet streak located downstream of an upper-level ridge feature. The DCB takes two paths as it descends: one is an anticyclonic path which forms a deformation zone behind 
a surface cold front (occasionally associated with a dry-line over the southern Great Plains); the other is a cyclonic path which ascends over the warmer air ahead of the cyclone (Figure 1.9). The overrunning of drier air in this region can lead to convective destabilization, which can increase precipitation development and cyclone strength.

The WCB originates in the warm, moist boundary layer and ascends anticyclonically over the surface warm front (i.e. isentropic lift) where it encounters the mid and upper-level Westerlies (Figure 1.9). This produces a broad stratiform cloud shield as the WCB overrides cooler and somewhat drier air below. The WCB typically displays the strongest vertical motions of the three main conveyor belts and is responsible for most the cyclone's meridional energy and moisture transport (Eckhardt et al. 2004). In addition to the classic anticyclonic branch of the WCB, Martin (1998) noted a branch that turned cyclonically with height into the occluded sector of the cyclone, forming a TROWAL airstream, short for "trough of warm air aloft" (Penner 1955). The TROWAL has been shown to contribute to mid-level frontogenesis and enhanced lift in the occluded sector where heavy snowfall is sometimes observed (Figure 1.9; Martin 1999; Ng 2005; Moore et al. 2005).

Perhaps the most complex and less agreed-upon conveyor belt is the $\mathrm{CCB}$, which originates in cool, sometimes moist boundary layer air (generally below $950 \mathrm{hPa}$ ) and travels westward toward the cyclone beneath the WCB. In the model developed by Carlson (1980), the $\mathrm{CCB}$ was shown to turn anticyclonically and rise into the mid and upper troposphere ahead of the cyclone (Figure 1.10). However, Schultz (2001) found through the use of back trajectories that the CCB is actually composed of both an anticyclonic and cyclonic path. In this revised model, the cyclonic path is located near the surface while the anticyclonic path is located vertically between the cyclonic path and the WCB. Because the anticyclonic path is 
located near the WCB and TROWAL airstreams, it is often associated with heavy

precipitation in the occluded sector of a mature cyclone (Moore et al. 2005). Schultz (2001) concluded that Carlson's choice of isentropic surfaces precluded him from identifying the cyclonic path of the $\mathrm{CCB}$ due to strong horizontal shear in the region between the $\mathrm{CCB}$ and WCB. In regions of high shear, nearby air parcel trajectories will be more likely to approach each other, so small-scale variations tend to be masked or integrated into a single airstream when plotted on an isentropic surface (Schultz 2001). In fact, Bierly (1999) concluded through use of isentropic analysis that the cyclonically-turning branch of the CCB was the main snowfall mechanism associated with Midwest cyclones. However, according to the results of Schultz (2001), the main mechanism was likely the anticyclonically-turning branch of the CCB along with contributions from the TROWAL airstream. Indeed, a subsequent study by Martin (1999) indicated that the TROWAL airstream was connected with the heaviest precipitation in the northwest sector of three occluded Midwestern cyclones. The primary role of the cyclonic path of the $\mathrm{CCB}$ may therefore be to maintain a lowertropospheric temperature profile supportive of winter precipitation (Schultz 2001; Moore et al. 2005).

\subsubsection{Summary}

This literature review raises a number of questions regarding winter precipitation that are applicable to central North Carolina:

- What are the relative contributions of adiabatic and diabatic processes to the resulting vertical temperature profile? In particular, what processes contribute to the changing temperature of air parcels that are advected into the elevated warm layer during 
different types of winter storms? Why are some elevated warm layers above freezing (resulting in freezing precipitation at the surface) and others are not?

- What are the source regions of the moisture used for precipitation formation? How much do these regions contribute to the evaporation-precipitation cycle (i.e. change in the absolute moisture content of the air) that result in the formation of heavy winter precipitation?

- What roles do the CCB and WCB play in the development of heavy winter precipitation? Is the traditional conveyor belt model sufficient for describing the airflow and transport pathways associated with winter precipitation-producing weather systems?

- How do the synoptic patterns vary between winter precipitation events characterized by differences in precipitation amount, precipitation type, moisture source region, and airflow configurations?

This dissertation project attempts to answer these and other related questions through the use of back trajectories. Interestingly, only one other study was identified that used trajectories to determine the ingredients and physical processes associated with winter precipitation. Moore et al. (2005) used high-resolution model data to produce mesoscale trajectories of airstreams connected with two heavy banded snowfall events in the Upper Midwest. They concluded that the "belts" of air portrayed in the trajectory models aided in the visualization of the features and processes associated with heavy banded snowfall, which is a major forecasting challenge in the region. In a similar vein, this dissertation project will use the HYSPLIT tool to diagnose the ingredients and physical processes associated with 
winter precipitation in central North Carolina and trace their evolution back across space and time. Additionally, this project will attempt to relate the space-time characteristics of the trajectories to the antecedent synoptic-scale circulation.

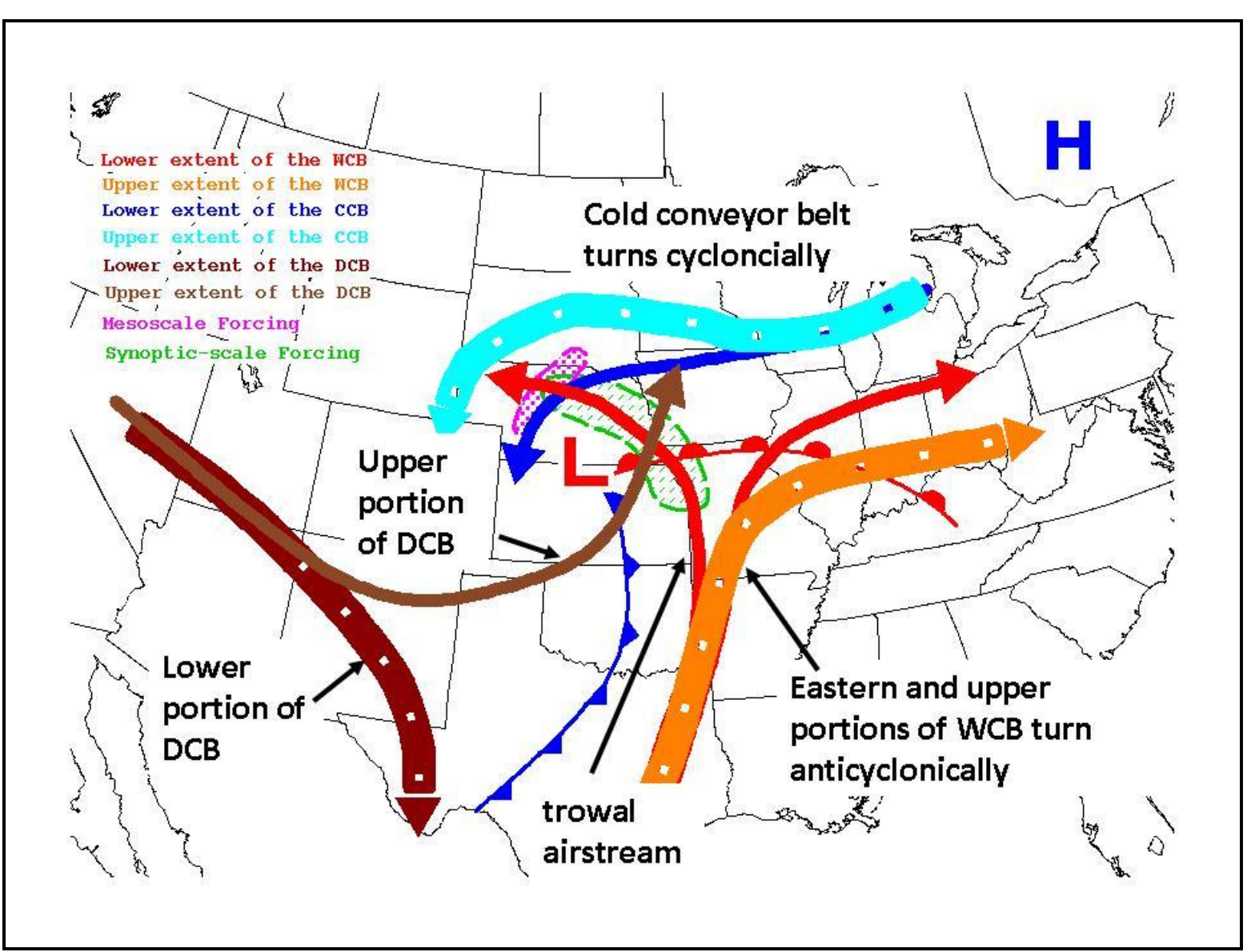

Figure 1.9. Schematic representation of the primary conveyor belts in a mature extra-tropical cyclone (from Ng 2005, his Figure 7.9). 


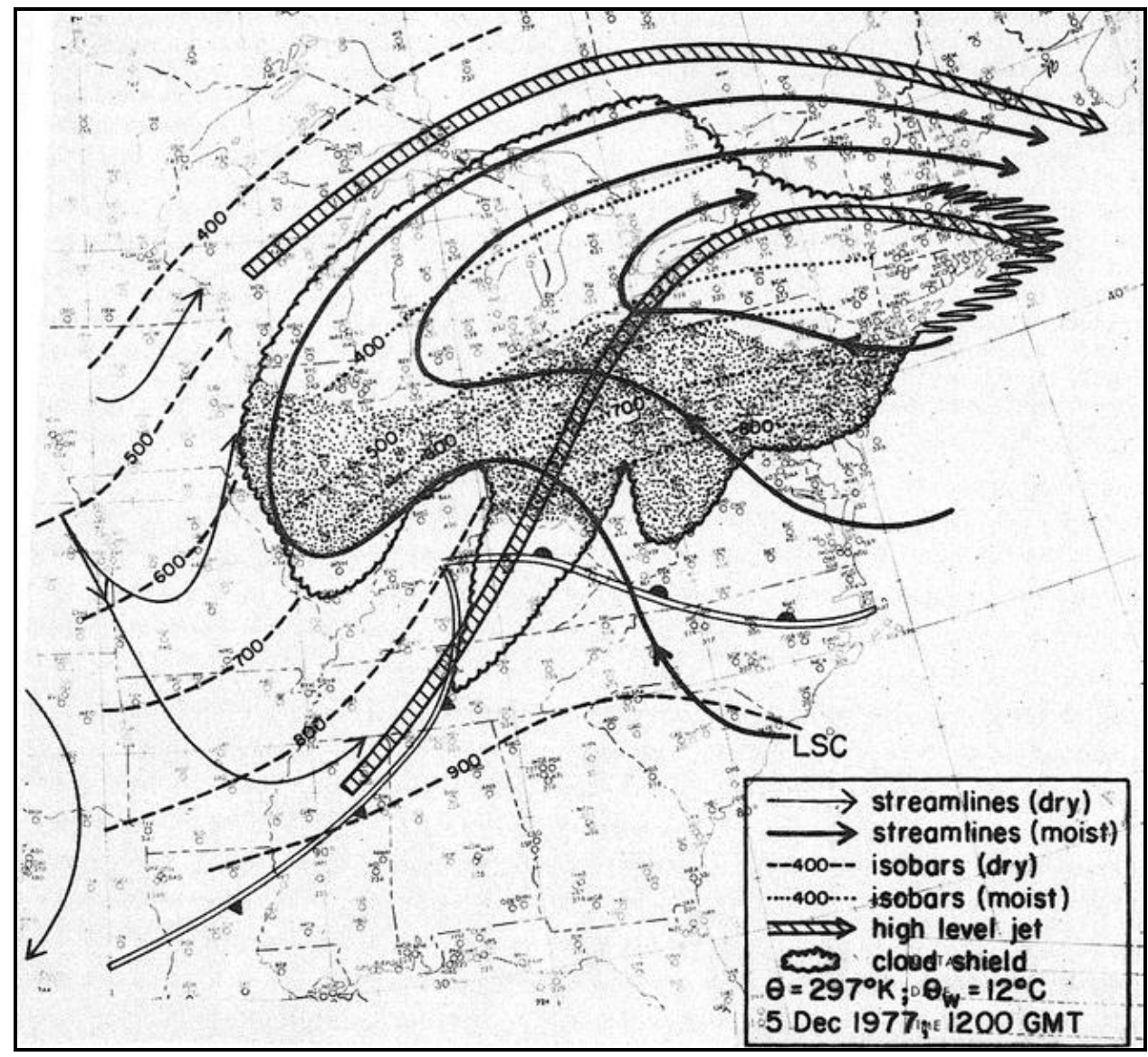

Figure 1.10. Depiction of the anticyclonic path of the cold conveyor belt using isentropic surfaces (solid black streamlines) (from Carlson 1980, his Figure 4). 


\subsection{Research Design}

\subsubsection{Research Questions}

The following research questions will guide this dissertation project:

1. What are the characteristics of air parcels that are advected into the atmospheric column of an evolving winter storm?

2. What is the space-time character of these air parcels as they approach the region of winter precipitation? Where do they originate? What changes are observed in their altitude with respect to time?

3. How does this space-time character affect the meteorological properties of air parcels (e.g. temperature, moisture) as they approach the region of winter precipitation? In particular, how does the Atlantic Ocean and Gulf Stream current affect the properties of the air parcels?

4. What are the physical processes (e.g. adiabatic cooling, diabatic warming) and upstream features that operate on the air parcels as they approach the region of winter precipitation?

5. What are the antecedent synoptic-scale circulation features that control the movement of the air parcels?

6. How do the identified characteristics of the air parcels relate to the intensity and type of winter precipitation observed? 


\subsubsection{Research Objectives}

The research questions above will be addressed with the following objectives in mind:

a. Identify heavy winter storms from precipitation maps and event summaries produced by the Raleigh NWS forecast office and classify events according to the dominant precipitation type (snow or freezing rain);

b. Compute model soundings of temperature, moisture, and vertical velocity (i.e. vertical profiles) for each storm at the location and time of heaviest precipitation;

c. Run 72 -hr backward trajectories at three critical levels: near-surface $(975 \mathrm{hPa})$, warm layer zone $(850 \mathrm{hPa})$, and dendritic growth zone (mean pressure between $-13^{\circ} \mathrm{C}$ and $-17^{\circ} \mathrm{C}$ ) to determine the three-dimensional air and moisture transport pathways and source regions;

d. Calculate key meteorological parameters at 1-hr intervals along each trajectory, including temperature, moisture, vertical velocity, potential temperature, and net diabatic contribution;

e. Compute ensemble and comparative trajectories for selected storms to assess the sensitivity of the trajectory models to the input data and identify trajectory characteristics that discriminate between locations with different precipitation types and/or intensities;

f. Relate the back trajectories to synoptic-scale circulation patterns by comparing composite plots across storm types with different precipitation and trajectory characteristics 


\section{Chapter 2}

\section{DATA AND METHODS}

\subsection{Overview}

This chapter describes the data and research methods, including the backward (i.e. upstream) trajectory analyses. It begins with a description of the study area, followed by the procedures used to: 1) select and classify winter storms; 2) compute model soundings and back trajectories; 3) calculate meteorological parameters along each trajectory segment; 4) compute ensemble and comparative trajectories for a sample of storms; and 5) relate the back trajectories to synoptic-scale circulation patterns. This chapter also provides an overview of atmospheric trajectories, as well as a description of the HYSPLIT tool and meteorological dataset used in the trajectory analyses.

\subsection{Study Area}

The study area encompasses the Raleigh NWS County Warning Area (CWA), a contiguous region covering 31 counties in central North Carolina (Figure 2.1). The CWA spans three physiographic regions - the Piedmont, Sandhills, and inner Coastal Plain - and ranges in elevation from approximately $1000 \mathrm{ft}(300 \mathrm{~m})$ in the west to approximately $100 \mathrm{ft}$ $(30 \mathrm{~m})$ in the east. The decision to select winter precipitation events from this region was based on several factors. First, the CWA is a highly populated area with several major urban and transportation corridors. According to the 2010 U.S. Census, the CWA is home to 
approximately 4.3 million people, which accounts for 45 percent of the state population. As a result, the societal impacts from winter storms in the region can be significant. These include economic losses, property damage, disruption to utilities and transportation, power outages, school and business closings, injury, and loss of life (Fuhrmann et al. 2009).

Second, the climatological position of the precipitation type transition zone (e.g. rainsnow line) extends across the CWA (Keeter et al. 1993). Therefore, most winter precipitation forecasts in the region are predicated on the accurate prediction of the transition zone and its characteristics (i.e. broad or narrow), which are tied to the vertical thermal profile (Keeter et al. 1995). Average annual snowfall across the CWA ranges from approximately 7 in $(17.8 \mathrm{~cm})$ in the west to 1 in $(2.5 \mathrm{~cm})$ in the east (Fuhrmann and Konrad 2005). With regard to freezing rain, the CWA experiences three to four events on average each year, with annual totals ranging from 0.6 in $(1.5 \mathrm{~cm})$ at Greensboro to 0.2 in $(0.5 \mathrm{~cm})$ at Fayetteville (Fuhrmann and Konrad 2005).

Finally, the Raleigh NWS has documented most winter storms affecting the CWA since 1977 and maintains an online archive of detailed precipitation maps and case studies (http://www.erh.noaa.gov/rah/events). These maps are produced using daily snowfall data from the Cooperative Observer (COOP) network as well as hourly precipitation data from first-order airport stations. This level of detail allows for more precise identification of locations with the heaviest precipitation and for demarcating regions of different precipitation types (i.e. freezing, frozen, and liquid precipitation). Moreover, while conceptual forecasting models have been developed from several detailed case studies of these storms, none have used trajectories or other airflow models to analyze the ingredients associated with winter precipitation. 


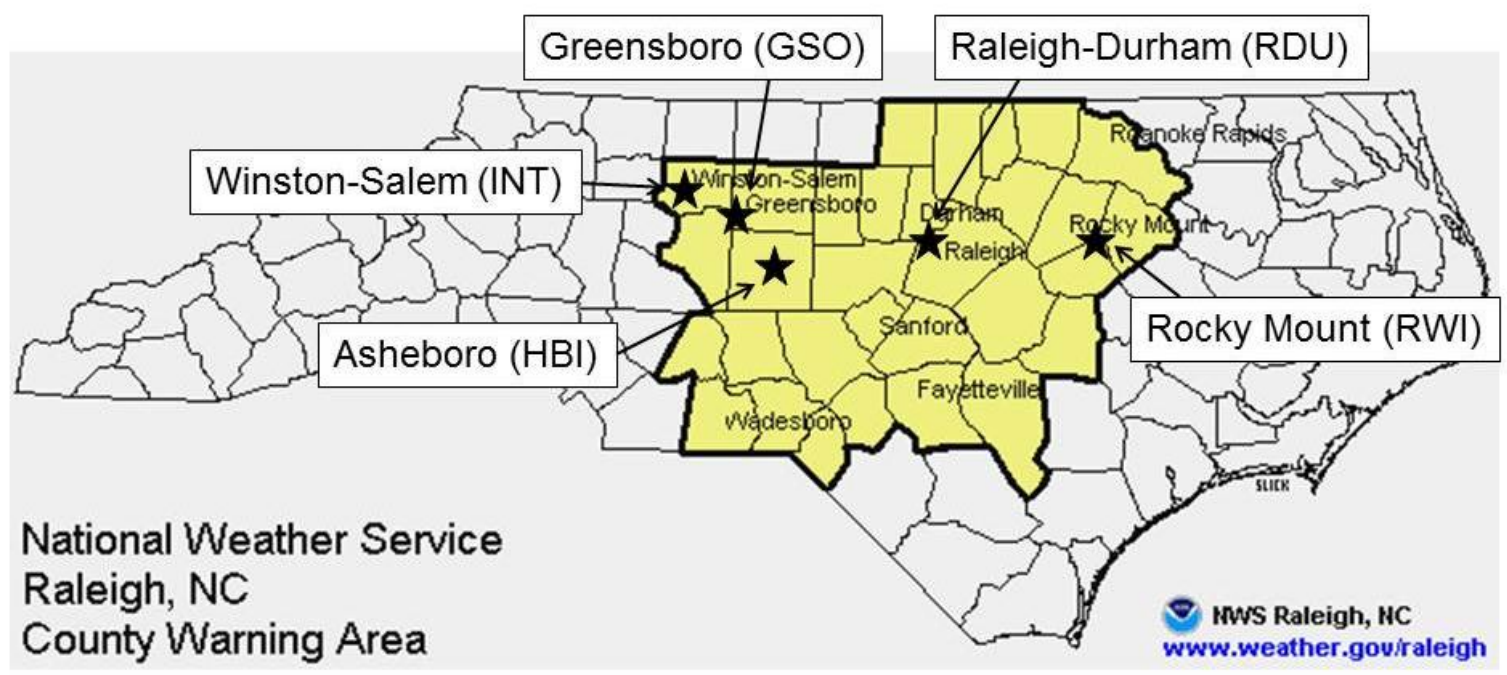

Figure 2.1. County-level map of North Carolina with the Raleigh NWS County Warning Area highlighted in yellow. Stars indicate the first-order stations and starting locations (with labels) for the sounding and trajectory analyses.

\subsection{Selection of Winter Storms}

Using the Raleigh NWS online archive of winter storms, the heaviest events from 2000 to 2010 were selected for analysis. This period was chosen for a few reasons. First, the level of detail of the precipitation maps produced by the Raleigh NWS has improved over the last decade due to the inclusion of more station observations. Second, the spatial resolution of the meteorological dataset used in the trajectory analyses is maximized during this period (see section 2.4.4). Finally, a total of 60 winter storms were documented by the Raleigh NWS over this period, providing a reasonably large sample from which the heaviest events could be selected for analysis. Only the heaviest events were examined because they were generally associated with the greatest societal impacts (Fuhrmann et al. 2009). Since the 
study area corresponds to the Raleigh CWA, the heaviest events were defined based on NWS criteria used to forecast and warn for heavy snow and ice (hereafter referred to as snowstorms and ice storms). In the Southeast U.S., most NWS offices, including Raleigh, $\mathrm{NC}$, define a snowstorm as one that produces at least 4 in $(10.2 \mathrm{~cm})$ of snow in at least one county within the CWA (B. Locklear, personal communication, 2009). In the case of an ice storm, the NWS will issue an Ice Storm Warning if at least 0.25 in $(0.6 \mathrm{~cm})$ of ice is expected to accumulate within the CWA. In cases where ice accumulation resulted from both freezing rain and sleet, the Raleigh NWS typically reported the combined total from both types on their precipitation maps. In these cases, the threshold for an ice storm, as defined in this study, was changed from 0.25 in $(0.6 \mathrm{~cm})$ to 0.5 in $(1.3 \mathrm{~cm})$.

Precipitation maps of the original 60 storms from 2000 to 2010 were examined to determine if at least one observation was made in the Raleigh NWS CWA that met the above criteria for either a snowstorm or ice storm. This yielded a total of eight snowstorms (Table 2.1) and seven ice storms (Table 2.2). Interestingly, none of the original 60 storms met both criteria (i.e. snowstorm and ice storm). Precipitation maps of these storms can be found in the appendix. At least 10 in $(25.4 \mathrm{~cm})$ of snow was recorded in six of the eight snowstorms, while two storms had reports of at least 20 in $(50.8 \mathrm{~cm})$. In addition, five of the seven ice storms recorded at least 1 in $(2.5 \mathrm{~cm})$ of ice accumulation from freezing rain and sleet. The duration of each storm was determined by examining the hourly weather observations and precipitation totals from the first-order station closest to the location of heaviest precipitation as indicated on the NWS precipitation maps (see Figure 2.1). Hourly data from the nearest first-order station were also examined to confirm the maximum storm-total precipitation for each storm. 
Table 2.1. List of snowstorms examined in this study. Locations for the sounding and trajectory analyses are shown in Figure 2.1

\begin{tabular}{|l|c|c|c|}
\hline \multicolumn{1}{|c|}{ Event } & $\begin{array}{c}\text { Location for } \\
\text { Trajectory } \\
\text { Analysis }\end{array}$ & $\begin{array}{c}\text { Maximum Event- } \\
\text { Total Precipitation } \\
\text { (in) }\end{array}$ & $\begin{array}{c}\text { Duration } \\
\text { (hr) }\end{array}$ \\
\hline 24-25 January 2000 & RDU & 20 & 22 \\
\hline 3-4 December 2000 & RWI & 10 & 12 \\
\hline 3-4 January 2002 & RDU & 14 & 27 \\
\hline 26-27 February 2004 & HBI & 20 & 18 \\
\hline 26 December 2004 & RWI & 10 & 13 \\
\hline 20 January 2009 & RDU & 8 & 10 \\
\hline 1-2 March 2009 & GSO & 7 & 20 \\
\hline 26-27 December 2010 & RWI & 14 & 13 \\
\hline
\end{tabular}

Table 2.2. List of ice storms examined in this study. Locations for the sounding and trajectory analyses are shown in Figure 2.1. Values in italics are for combined sleet and freezing rain totals.

\begin{tabular}{|l|c|c|c|}
\hline \multicolumn{1}{|c|}{ Event } & $\begin{array}{c}\text { Location for } \\
\text { Trajectory } \\
\text { Analysis }\end{array}$ & $\begin{array}{c}\text { Maximum Event- } \\
\text { Total Precipitation } \\
\text { (in) }\end{array}$ & $\begin{array}{c}\text { Duration } \\
\text { (hr) }\end{array}$ \\
\hline 30 January 2000 & GSO & 0.75 & 24 \\
\hline 4-5 December 2002 & RDU & 1.50 & 18 \\
\hline 16-17 February 2003 & INT & 3.00 & 30 \\
\hline 26-27 February 2003 & GSO & 1.00 & 32 \\
\hline 14 December 2003 & GSO & 1.00 & 24 \\
\hline 25-26 January 2004 & GSO & 6.00 & 16 \\
\hline 29-30 January 2005 & GSO & 0.25 & 24 \\
\hline
\end{tabular}


Because winter storms were selected on the basis of precipitation amount, it is important to recognize the limitations associated with snowfall and ice accumulation measurements. Most snowfall observations are made by volunteers once every 24 hours, and while the NWS has developed standardized measuring procedures to ensure consistency among all observers, some inter-observer variability can occur (Perry 2006). Since the storms in this study were separated by at least 24 hours, variations in the reporting time of daily (i.e. 24-hr) snowfall (Perry 2006) were not likely to have an effect on the values reported in the precipitation maps. Other factors and processes that may influence snowfall measurements include sublimation, compaction, melting, blowing and drifting, snow density, mixed precipitation, and event duration (Kocin and Uccellini 2004).

Observations of freezing rain and sleet are typically made at first-order stations that report hourly precipitation and present weather conditions. Beginning in the early $20^{\text {th }}$ century, these observations were made by NWS observers (Changnon 2004). However, as part of the NWS modernization program in the mid-1990s, these NWS observers were replaced by automated surface observing sensors, or ASOS. In the CWA, several of these ASOS stations are equipped with an icing sensor that uses a small cylindrical probe to detect ice mass during periods of freezing rain (see Jones et al. 2004 for a description of the sensor and list of operating stations). A series of algorithms are then used to translate the ice mass into a radial ice thickness as well as an ice depth on a planar horizontal surface. The latter is used in standard METAR reports to identify freezing precipitation. These reports are then used to construct hourly time series of weather conditions and precipitation amounts at each first-order station. In addition to observations made at first-order stations, freezing rain and sleet are also reported by volunteer observers in the COOP network as well as by law 
enforcement and other government officials (e.g. emergency management). These reports are typically submitted to the respective NWS office either during or after an event as a Local Storm Report, or LSR. These reports can provide valuable information on the small-scale distribution and intensity of freezing precipitation as an event unfolds. As with snowfall, the NWS has published standardized guidelines for COOP observers who measure ice accumulation, but the consistency of these measurements is difficult to assess.

Since the surface temperature must be below freezing in order for ice to accumulate, weather stations that are located in areas prone to the effects of the urban heat island will observe fewer days with freezing rain and lower freezing rain amounts compared to nearby rural stations. Changnon (2003) found that large urban areas experience 15 to 40 percent fewer days with freezing rain compared to nearby rural areas. Therefore, it is likely that reductions in freezing rain due to local heating effects occurred around several of the urban weather stations in the Raleigh CWA.

\subsection{Trajectory Analysis}

\subsubsection{Theory}

Air motions can be viewed from one of two perspectives: Eulerian or Lagrangian (Dutton 1986; Stohl 1998). To help conceptualize these perspectives, imagine a ball floating down a swiftly moving river. In a Eulerian framework, the movement and position of the ball in the river would be viewed from the river bank at fixed points in space and time. In a Lagrangian framework, the vantage point would be from the ball itself as it floats down the river, noting its movement and position as it moves through space and time (i.e. its trajectory). Therefore, in the context of atmospheric motion, a Eulerian perspective focuses 
on fixed points in space and time, while a Lagrangian perspective focuses on individual air parcels as they move through space and time. The paths that these air parcels take as they travel through the atmosphere are referred to as trajectories.

Atmospheric trajectories are typically calculated from gridded wind and pressure fields and represent the approximate flow path of an air parcel. Computationally, the trajectory of an air parcel is defined by the differential trajectory equation (from Stohl 1998):

(1) $\frac{d \mathbf{X}}{d t}=\mathbf{X}^{\prime}[\mathbf{X}(t)]$

where $t$ is time, $\mathbf{X}$ is the position vector and $\mathbf{X}^{\prime}$ is the wind velocity vector. One can write

(2) $\mathbf{X}_{0}\left(t=t_{0}\right)=\mathbf{X}_{0}(\mathbf{X}, t)$

which gives the initial coordinates of parcel $\mathrm{X}_{0}$ at time $\mathrm{t}_{0}$. Therefore, Equation 2 can be used to determine the path of the air parcel either forward in time (i.e. forward trajectories, $t>t_{0}$ ) or backward in time (i.e. backward trajectories, $\mathrm{t}<\mathrm{t}_{0}$ ).

Atmospheric trajectories have been used to study dynamical processes in the atmosphere for several decades, beginning with the graphical techniques of Petterssen (1940). One of the earliest computational studies was conducted by Reap (1972), who used a primitive numerical model to compute trajectories to help verify wind forecasts during a severe weather event. Trajectory analysis has also been used to study airflow within extratropical cyclones using output from model simulations. Uccellini et al. (1985) calculated three-dimensional trajectories in a study of the President's Day snowstorm of 1979, while 
Mass and Schultz (1993) and Wernli and Davies (1997) analyzed backward trajectories of model-simulated cyclones to identify the main "belts" of air associated with cyclonic weather systems (i.e. the conveyor belt model). More recently, trajectory analysis has been used to study the inflow and outflow transport paths associated with a mature hurricane (Cram et al. 2007).

Trajectories have also been used to characterize large-scale circulation and air mass transport patterns at specific locations (i.e. trajectory or flow climatologies). Some of these studies subjectively classify trajectories based on manual analysis of their source region, curvature, length, or meteorological properties (e.g. Dayan 1986), while other studies use more objective clustering techniques to numerically identify trajectory classes (e.g. Dorling et al. 1992; Hondula et al. 2009). Recent research has employed trajectories to assess water vapor transport and identify remote sources of moisture (e.g. Bao et al. 2006; Knippertz and Martin 2007; Roberge et al. 2009; Milrad et al. 2010). These studies tied transport paths to the occurrence of large-scale circulation features such as warm conveyor belts and jet streams (Figure 2.2). Trajectory analyses have been used most frequently in the study of atmospheric chemistry and air quality, specifically to illustrate the dispersion patterns and concentration of chemical compounds and aerosols and identify their source regions (see references in Hondula et al. 2009).

It is important to distinguish trajectories from streamlines, which are identified on isentropic surfaces (i.e. surfaces of equal potential temperature) and are commonly used in conveyor belt studies (Bierly and Winkler 2001). Streamlines represent the direction of airflow at fixed points in time and therefore determine the path of an air parcel trajectory at these fixed points. In fact, under steady state or quasi-stationary conditions (e.g. a slow- 
moving anticyclone), streamlines and trajectories will be parallel. However, in situations where the circulation is changing rapidly (e.g. a deepening mobile cyclone), streamlines and trajectories will diverge as the streamlines adjust to the flow and the local vertical velocity is no longer zero (Schultz 2001).

\subsubsection{Limitations}

A review of the trajectory literature by Stohl (1998) revealed some important limitations to trajectory analysis and potential sources of error. Trajectory calculations typically rely on gridded output from numerical models, the most basic being diagnostic wind models that interpolate between radiosonde observations. Such a coarse domain may adequately depict upper-level trajectories that are near geostrophy, but may be inadequate in depicting boundary layer trajectories or those interacting with complex terrain. Over time, improvements in the vertical and horizontal resolution of numerical weather prediction models have resulted in more accurate representations of boundary layer and other mesoscale trajectories. In fact, research by Draxler (1996) and Jorba et al. (2004) suggest that threedimensional kinematic trajectories may be more accurate than isentropic analysis when accurate vertical wind fields are available.

Potential sources of error in trajectory analysis include truncation, a shortening of the time steps and/or reduction in the number of grid cells used in the model, as well as interpolation, vertical wind calculations, wind field errors, and initial conditions (e.g. trajectory starting location, model physics) (Stohl 1998). Traditionally, the accuracy of a trajectory model has been assessed by constructing a reference trajectory, or tracer, using either balloon launches (e.g. Draxler 1996) or material tracers, such as smoke plumes (e.g. 
Draxler 1991), pollen (Raynor et al. 1983), and volcanic ash (e.g. Heffter and Stunder 1993). Mechanical tracers, such as potential vorticity, are also useful in assessing the magnitude of trajectory errors resulting from the error sources listed above. For example, Kahl and Samson (1988) and Maryon and Heasman (1988) found that interpolation errors for trajectories calculated in environments with significant convection and high shear were as much as $500 \mathrm{~km}$ over 72 hours, or approximately 50 percent of the distance traveled. In a similar study, Stohl and Seibert (1997) assessed the relative accuracy of a large set of trajectories and found position errors of up to 20 percent for trajectories lasting longer than 24 hours. A popular method to test the sensitivity of a trajectory model to the meteorological input data is the ensemble (Draxler 2003). This method involves performing multiple model runs with slightly different initial conditions and then assessing the variance in the output trajectory characteristics. 


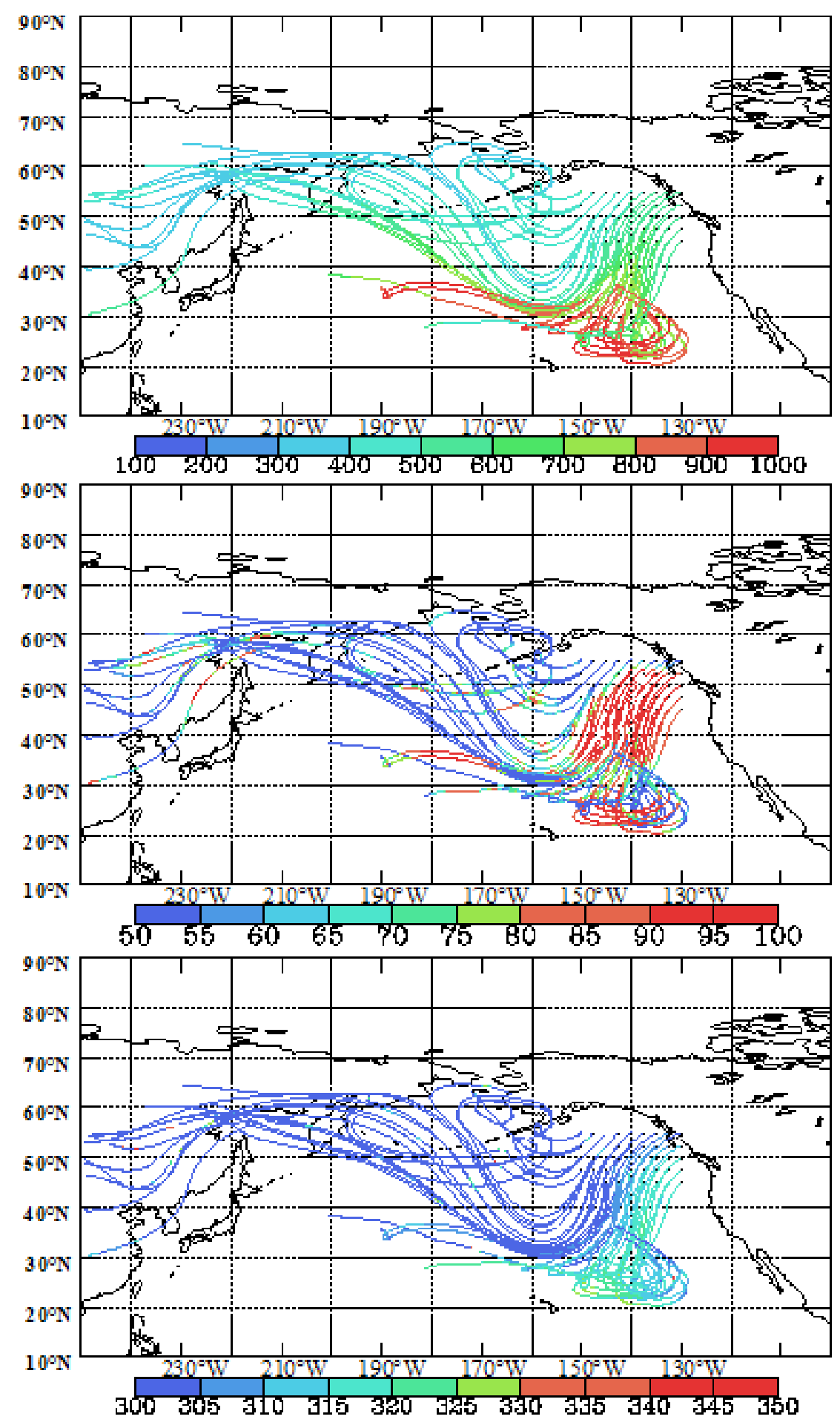

Figure 2.2. A set of 5-day backward trajectories illustrating an intense water vapor transport event into western Canada. Coloring represents various parameters calculated along each trajectory segment: (top) pressure (hPa); (middle) relative humidity (percent); and (bottom) equivalent potential temperature (K). From Roberge et al. (2010), their Figure 2. 


\subsubsection{HYSPLIT Description}

In this study, back trajectories were calculated using Version 4.9 of the Hybrid Single-Particle Lagrangian Integrated Trajectory (HYSPLIT) tool (Draxler and Rolph 2011). The initial version of the HYSPLIT was developed in the early 1980s at NOAA's Air Resource Laboratory (ARL) and used only weather balloon observations to calculate trajectories (Draxler and Taylor 1982). The initial parcel dispersion models assumed uniform vertical mixing in the daytime and no mixing at night. The second version was developed in the late 1980s and introduced a diffusivity profile that better resolved the variability in daytime mixing and was able to account for small amounts of mixing at night (Draxler and Stunder 1988). The third version introduced the use of gridded forecast routines developed from numerical weather prediction models, a major improvement over the first two versions (Draxler 1992). The fourth version became operational in 1997 (Draxler and Hess 1998) and includes improved advection algorithms with better temporal interpolation, refinements in the equations used to compute the strength of vertical mixing, improvements in the calculation of horizontal dispersion by accounting for small-scale deformation in the wind field, and improvements to the graphical user interface.

Most trajectory models use meteorological datasets from either NCEP or the European Centre for Medium-Range Weather Forecasts (ECMWF). Models developed internationally typically use ECMWF datasets and include LAGRANTO, TRAJKS, and FLEXTRA (see Stohl et al. 2001 for descriptions of these trajectory models). Though other trajectory tools have been developed for the continental U.S. (e.g. White et al. 2006), the HYSPLIT tool remains the most popular tool for studies across North America due to its ease of use, improvements in its dispersion algorithms, and its ability to calculate trajectories from 
several different meteorological datasets. For this study, the HYSPLIT tool was run interactively using the ARL's Real-time Environmental Applications and Display (READY) system (Rolph 2011). The online version includes a user-friendly interface that allows the user to choose from several different meteorological models. The HYSPLIT tool can also be downloaded to a Windows or Mac PC, but the user must obtain their own meteorological data files.

\subsubsection{Meteorological Dataset}

The meteorological dataset used in this study is the NCEP's Eta Data Assimilation System (EDAS). The EDAS is commonly used in trajectory studies and combines meteorological observations with short-term weather forecasts to produce dynamically consistent estimates of the state of the atmosphere. The EDAS contains several basic fields, including the $\mathrm{u}$ and $\mathrm{v}$ components of the horizontal wind, temperature, and relative humidity that are archived every 3 hours across a North American grid extending from $12.2^{\circ} \mathrm{N}$ to $57.3^{\circ} \mathrm{N}$ latitude and from $49.4^{\circ} \mathrm{W}$ to $133.5^{\circ} \mathrm{W}$ longitude (Figure 2.3 ). The ARL saves each 3hr analysis to produce a continuous data archive.

The ARL began archiving EDAS data in 1997 with a spatial resolution of $80 \mathrm{~km}$. In 2004, the spatial resolution increased to $40 \mathrm{~km}$. Archived fields in EDAS are output onto 26 vertical pressure surfaces from 1000 (or surface pressure) to $50 \mathrm{hPa}$. The vertical resolution from 1000 to $700 \mathrm{hPa}$ is $25 \mathrm{hPa}$, while the resolution from 700 to $50 \mathrm{hPa}$ is $50 \mathrm{hPa}$. Hondula et al. (2009) used the EDAS to examine a large sample of trajectories in HYSPLIT during a four month overlap period in the datasets (January to April 2004) and found no statistically significant differences in the horizontal or vertical extents of the trajectories. Therefore, both 
versions were used in this study. The higher resolution dataset was used for events that occurred during the overlap period.

The use of the EDAS over other available datasets in the ARL archive was based on two factors. First, the EDAS provided the highest model resolution over the study period (2000 to 2010). The ARL recently added the $12 \mathrm{~km}$ North American Mesoscale Model (NAM), but this archive only extends back to 2007. Second, the EDAS is an integral part of the operational Eta Forecast Model (Black 1994), which has been used routinely to forecast winter precipitation in the Raleigh CWA since the early 1990s (B. Locklear, personal communication, 2009). By using the EDAS, and therefore maintaining consistent model physics and parameterizations, the results of this study should be directly applicable to forecasters at the Raleigh NWS.

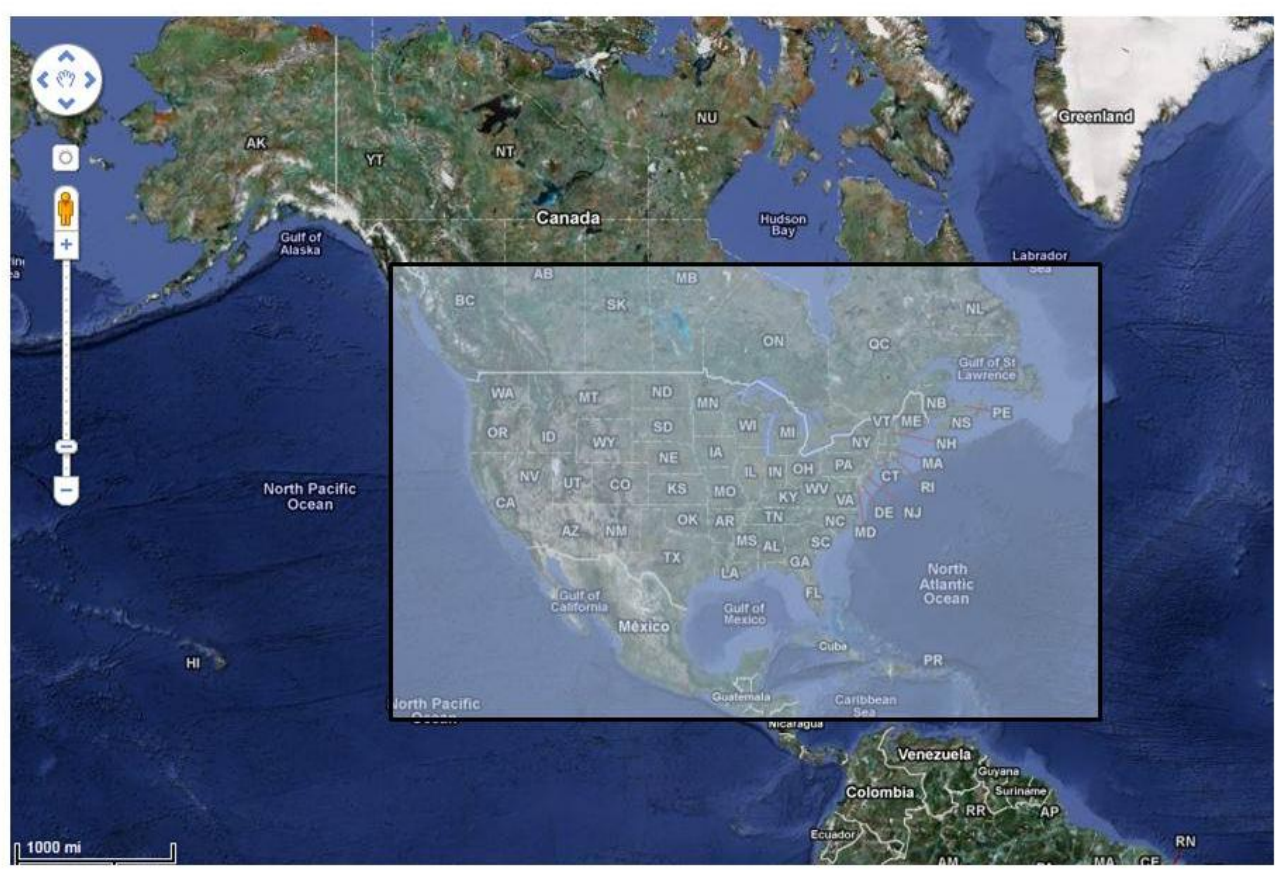

Figure 2.3. The EDAS grid domain displayed in Google Maps. 


\subsection{Research Methods}

\subsubsection{Model Soundings}

The first step in this research was to determine the vertical atmospheric profile for each storm at the time and location of heaviest winter precipitation. The location of heaviest precipitation was determined by examining the NWS precipitation map for each storm. These locations are provided in Figure 2.1 and in Tables 2.1 and 2.2. The time of heaviest precipitation was determined by examining the hourly precipitation time series for the firstorder station nearest the location of heaviest precipitation. Specifically, the time (hour) of heaviest precipitation was identified as the middle hour of the 3-hr period with the heaviest aggregate precipitation in the time series. An example of this procedure is shown in Table 2.3. This procedure was used because more than 50 percent of the total precipitation observed over most of the events in this study occurred in a 3-hr period of time (Figure 2.4).

To assess how well the EDAS dataset resolved the vertical thermal profile during heavy winter precipitation, and therefore evaluate how well the back trajectories resolved the wintertime atmosphere upstream of the precipitation region, model soundings of air temperature for four events (two snowstorms and two ice storms) were computed and compared against the corresponding soundings from radiosonde launches at Greensboro, NC (GSO; see Figure 2.1).

In general, there was excellent agreement between the modeled and observed thermal profile above approximately $800 \mathrm{hPa}$ (see appendix). However, the modeled soundings were warmer than the observed soundings between approximately 800 and $950 \mathrm{hPa}$. This was unexpected, as the Eta Forecast Model was updated in 2001 to account for the latent heat absorption (i.e. cooling) resulting from the melting and/or evaporation of falling snow and 
rain (Rogers et al. 2001). One explanation may be that the model overestimated the magnitude of warm-air advection, which could compensate for the cooling due to melting and evaporation. In the two ice storm samples, which were associated with prominent CAD signatures, the modeled soundings produced a warmer and deeper melting layer $\left(>0^{\circ} \mathrm{C}\right)$ but failed to resolve the coldest portion of the surface-based cold wedge. Stohl and Seibert (1998) showed that some trajectory models do not adequately resolve the ageostrophic wind component in the bottom half of the troposphere. Since CAD events in central North Carolina result from an agestrophic adjustment of the low-level flow as it encounters the Southern Appalachians, the EDAS may be underestimating the strength of the ageostrophic wind and, therefore, the magnitude of cold-air advection in the lowest levels. There was generally good agreement in the near-surface thermal profile between the modeled and observed soundings. This was encouraging, as improvements to the Eta Land Surface Model (LSM) have resulted in more accurate surface temperature forecasts during periods of freezing precipitation (B. Locklear, personal communication, 2009).

Keeping these biases in mind, model soundings of temperature $\left({ }^{\circ} \mathrm{C}\right)$, moisture (mixing ratio, $\mathrm{g} / \mathrm{kg}$ ), and vertical velocity $(\mathrm{cm} / \mathrm{s})$ were constructed for each snowstorm and ice storm at the location and time of heaviest precipitation. These parameters were calculated at each of the 21 standard pressure levels in the EDAS dataset from 1000 to 300 hPa. The resulting vertical profiles were used to help determine the critical levels from which back trajectories were calculated. 
Table 2.3. Example of the procedure used to identify the time of heaviest precipitation. The 3 -hr period with the heaviest aggregate precipitation ( 0.33 in liquid equivalent) is highlighted in the Precipitation column with the middle hour (07 UTC) highlighted in the Time column.

\begin{tabular}{|c|c|c|c|}
\hline Date & $\begin{array}{c}\text { Time } \\
\text { (UTC) }\end{array}$ & $\begin{array}{l}\text { Precipitation } \\
\text { (in liquid } \\
\text { equivalent) }\end{array}$ & Present Weather* \\
\hline $01 / 24 / 2000$ & 23 & $\operatorname{Tr}$ & $-\mathrm{RA}$ \\
\hline $01 / 24 / 2000$ & 00 & 0.02 & -RA PL \\
\hline $01 / 25 / 2000$ & 01 & 0.04 & -SN BR \\
\hline $01 / 25 / 2000$ & 02 & 0.06 & -SN PL \\
\hline $01 / 25 / 2000$ & 03 & 0.06 & -SN BR \\
\hline $01 / 25 / 2000$ & 04 & 0.05 & -SN BR \\
\hline $01 / 25 / 2000$ & 05 & 0.06 & -SN BR \\
\hline $01 / 25 / 2000$ & 06 & 0.15 & +SN FZFG \\
\hline $01 / 25 / 2000$ & 07 & 0.10 & $+\mathrm{SN}$ FZFG \\
\hline $01 / 25 / 2000$ & 08 & 0.08 & $+\mathrm{SN}$ FZFG \\
\hline $01 / 25 / 2000$ & 09 & 0.08 & + SN FZFG \\
\hline $01 / 25 / 2000$ & 10 & 0.06 & + SN FZFG \\
\hline $01 / 25 / 2000$ & 11 & 0.00 & $+\mathrm{SN}$ FZFG \\
\hline $01 / 25 / 2000$ & 12 & $\operatorname{Tr}$ & -SN BR \\
\hline $01 / 25 / 2000$ & 13 & 0.02 & + SN FZFG \\
\hline $01 / 25 / 2000$ & 14 & $\operatorname{Tr}$ & SN FZFG \\
\hline $01 / 25 / 2000$ & 15 & 0.01 & SN FZFG \\
\hline $01 / 25 / 2000$ & 16 & 0.00 & -SN BR \\
\hline $01 / 25 / 2000$ & 17 & $\operatorname{Tr}$ & -SN BR \\
\hline $01 / 25 / 2000$ & 18 & 0.00 & -SN BR \\
\hline $01 / 25 / 2000$ & 19 & 0.01 & -SN BR \\
\hline
\end{tabular}

$* \mathrm{RA}=$ rain $; \mathrm{BR}=$ mist $; \mathrm{PL}=$ sleet $\mathrm{SN}=$ snow $; \mathrm{FZFG}=$ freezing fog 


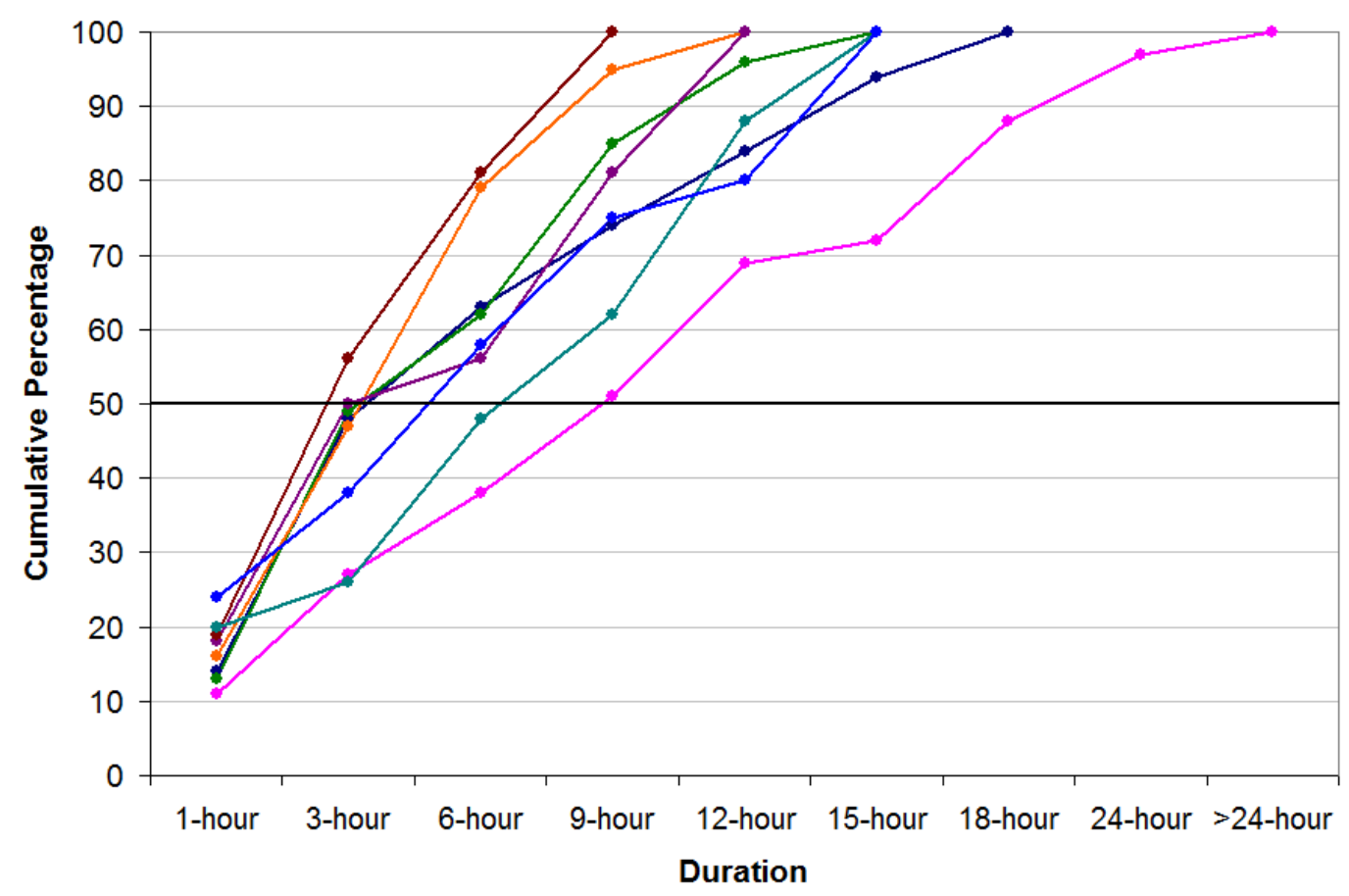

(A)

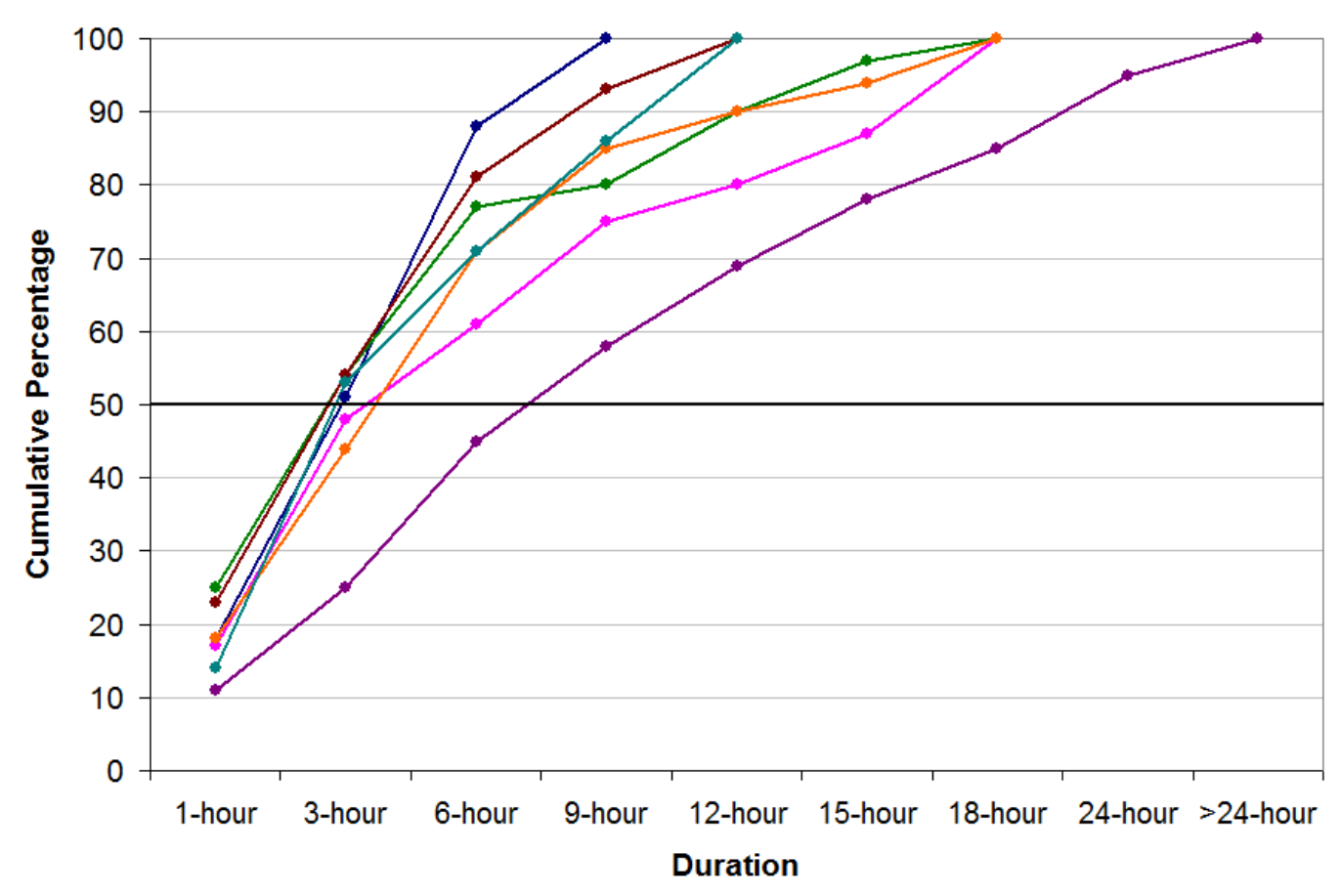

(B)

Figure 2.4. Percentage of the total precipitation observed across different time periods relative to the full duration of each (A) snowstorm and (B) ice storm. 


\subsubsection{Trajectory Calculations}

A backward trajectory analysis was undertaken for each snowstorm and ice storm in order to assess the influence of the upstream environment on the ingredients (i.e. temperature, moisture, lift) and physical processes that contribute to winter precipitation in central North Carolina. The HYSPLIT tool was used to calculate 72-hr back trajectories for air parcels at three critical levels over the location of heaviest precipitation. The 72-hr duration was selected to appropriately measure synoptic and sub-synoptic scale circulation patterns without introducing excessive errors in the trajectory estimates that may arise over longer time scales.

The three critical levels are associated with near-surface trajectories (ending at 975 $\mathrm{hPa}$ ), warm layer zone trajectories (ending at $850 \mathrm{hPa}$ ), and dendritic growth zone trajectories (ending at the mean pressure with temperature between -13 and $-17^{\circ} \mathrm{C}$ where precipitation production is especially high). Trajectories associated with the near-surface level reveal the circulation within the low-level cold wedge that is typically present during winter precipitation in the region. The decision to use the $975 \mathrm{hPa}$ level was based on examination of the modeled soundings, which showed good agreement with the observed soundings at that level. The $850 \mathrm{hPa}$ level was chosen to represent the warm layer zone because it is a standard pressure level used routinely to forecast precipitation type. Above (below) freezing temperatures in the warm layer zone at $850 \mathrm{hPa}$, as indicated in the modeled soundings, were associated with ice storms (snowstorms). Determining the processes and upstream features that contribute to these warm layer zone temperatures is a major goal of this research. Lastly, trajectories associated with the dendritic growth zone reveal the properties of the air associated with ice crystal formation growth. It has been noted that ice crystal growth in the 
dendritic range, when combined with sufficient moisture and upward vertical motion, can lead to heavy snowfall (Auer and White 1982; Perry 2006). Therefore, trajectories calculated at this level can aid in determining the processes and upstream features that contribute to the formation of precipitation in the wintertime atmosphere over central North Carolina.

The hourly latitude, longitude, and vertical coordinates associated with each back trajectory were used to develop composite trajectories for snowstorms and ice storms at each of the three critical levels. These composites were created by calculating the median coordinates among all events at 1-hr trajectory segments. Similar composites were also constructed for several meteorological variables along each trajectory. These included the quantities used to construct the modeled sounding profiles (i.e. temperature, moisture, vertical velocity) as well as the potential temperature $(\theta)$. Potential temperature is the temperature of an air parcel if it were compressed or expanded adiabatically from its current pressure to $1000 \mathrm{hPa}$. It is a conservative property in the absence of diabatic processes. When diabatic processes are occurring (e.g. melting, freezing, condensation, evaporation), the adiabatic assumption is negated and the air parcel will begin to move along a different potential temperature, or isentropic surface (i.e. the potential temperature of the parcel will change).

While examination of the potential temperature can indicate where diabatic processes are likely taking place along the trajectory, it cannot reveal on its own the relative contribution of adiabatic or diabatic processes to the resulting air parcel temperature. Therefore, a procedure was devised to calculate the amount of adiabatic warming and cooling relative to the amount of diabatic warming and cooling for air parcels traveling along each trajectory segment. The steps used in this procedure are outlined in Figure 2.5. As air 
parcels move vertically through the atmosphere, they warm and cool dry-adiabatically at a fixed rate of $10^{\circ} \mathrm{C} / 1000 \mathrm{~m}$ (i.e. the dry-adiabatic lapse rate, or DALR). If the change in air parcel temperature from one hour to the next along a given trajectory matches the DALR, then the parcel is assumed to have warmed or cooled adiabatically with no contributions from diabatic processes. If the change in temperature does not match, then the residual is considered to be a result of diabatic processes.

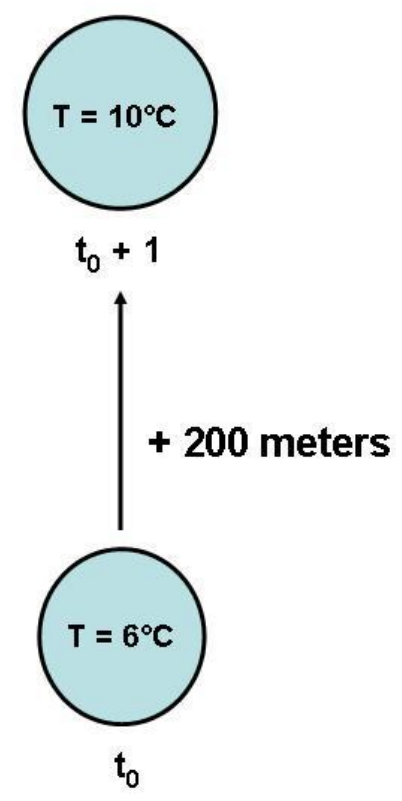

\begin{tabular}{ll|}
\hline $\begin{array}{l}\text { Temperature Change } \\
\text { according to DALR } \\
\text { (adiabatic contribution): }\end{array}$ & $-2^{\circ} \mathrm{C}$ \\
$\begin{array}{l}\text { Observed temperature } \\
\text { change: }\end{array}$ & $+4^{\circ} \mathrm{C}$ \\
\hline $\begin{array}{l}\text { Difference/Residual } \\
\text { (diabatic contribution): }\end{array}$ & $+6^{\circ} \mathrm{C}$ (latent warming) \\
\hline
\end{tabular}

Figure 2.5. Example of the procedure used to calculate adiabatic and diabatic contributions to the change in temperature of an air parcel between two trajectory segments $\left(t_{0}\right.$ and $\left.t_{0}+1\right)$. 
In order to distinguish between the trajectories at locations with different precipitation types and intensities in a given event, a set of comparative back trajectories were calculated for four of the events (two snowstorms and two ice storms). These comparative trajectories were calculated to determine which of the ingredients (i.e. temperature, moisture, lift) and processes (i.e. adiabatic or diabatic) best distinguishes between different precipitation types and intensities in a given event.

An ensemble trajectory analysis was employed using the same four events from the comparative analysis described above to test the sensitivity of the trajectory calculations to the meteorological data. The technique developed by Draxler (2003) was used to generate multiple trajectories (i.e. members) from the EDAS datasets. This was done to account for various types of trajectory errors and uncertainties, which can include spatial and temporal resolution, sampling errors, and oversimplification of meteorological conditions and physical processes. Each ensemble member was calculated from the same ending time and location, but during the calculation the meteorological grid was offset by one grid point in the horizontal $(\mathrm{X}, \mathrm{Y})$ direction and by $250 \mathrm{~m}$ in the vertical $(\mathrm{Z})$ direction, resulting in an ensemble of 27 members. The ensembles were computed from the same critical levels for the same set of meteorological properties as in the other trajectory analyses. The ensemble analysis reveals how variations in the space-time character and source regions of the trajectories, due to uncertainties in the calculations, affect the meteorological properties of the air advected into the region of heavy precipitation. Estimation of adiabatic and diabatic processes using the ensemble approach can also provide insight into the ability of the trajectory model to resolve these important aspects of winter precipitation. 


\subsubsection{Synoptic Analysis}

To determine the synoptic-scale circulation patterns associated with snowstorms and ice storms and their trajectory characteristics, composite plots were constructed of the lower and middle-tropospheric geopotential height fields (i.e. 1000, 850 and $500 \mathrm{hPa}$ ). These plots were constructed at 24-hr intervals beginning at the end of the back trajectory (T-72 hours) and ending at the start of the trajectory ( $\mathrm{T}-0$ hours). The geopotential height fields were plotted using data from the North American Regional Reanalysis (NARR; Mesinger et al. 2006). The NARR was developed at NCEP and is a long-term, dynamically consistent, dataassimilation-based collection of climate data for North America from 1979 to the present. The NARR was produced with high spatial (32 km), vertical (45 layers) and temporal (3 hourly) resolution and is based on a version of the Eta Forecast Model and the EDAS. Analysis of geopotential height fields was performed using software created by NOAA's Earth System's Research Laboratory (http://www.esrl.noaa.gov/psd/). 


\section{Chapter 3 \\ SNOWSTORMS}

\subsection{Overview}

This chapter describes the results of the back-trajectory and synoptic analyses conducted for eight major snowstorms that occurred in central North Carolina from 2000 to 2010. It begins with a discussion of the vertical profiles of temperature, moisture, and vertical velocity at the time and location of heaviest snowfall. The second section discusses the results of the composite trajectory analysis, which focuses on the space-time character and meteorological properties of air parcels associated with three critical vertical pressure levels: the near-surface, the warm layer zone, and the dendritic growth zone. Adiabatic and diabatic contributions to air parcel temperature and moisture content are also calculated along each trajectory segment to assess the physical processes connected with heavy snowfall in the region. This section also examines a set of comparative and ensemble trajectories for a sample of snowstorms to determine the trajectory characteristics that distinguish between locations and times of different precipitation type and intensity and assess the sensitivity of the trajectory calculations to the meteorological input data. The third, and final section, discusses the results of the synoptic analysis, which focuses on the relationship between the large-scale antecedent circulation and the space-time character of the composite back trajectories. 


\subsection{Modeled Vertical Profile}

Using the EDAS model output data, vertical profiles of temperature, moisture, and vertical velocity were constructed for each snowstorm at the time and location of heaviest snowfall. Each parameter was calculated at each of the 21 vertical pressure levels in the EDAS datasets.

All of the modeled temperature profiles (Table 3.1) revealed freezing or subfreezing air at each pressure level and a low-level inversion of varying depth (between 25 and 100 $\mathrm{hPa}$ ) and temperature increase (between 0.5 and $2.0^{\circ} \mathrm{C}$ ). The height of the inversion ranged from 925 to $775 \mathrm{hPa}$. As described earlier, the air advected into the warm layer zone (WLZ) was modeled by back-trajectories at the $850 \mathrm{hPa}$ level, which falls near the middle of the range of inversion heights in the snowstorm sample. Two of the eight profiles exhibited nearfreezing isothermal layers within the inversion. These likely developed in response to diabatic cooling from melting snowflakes. The contribution of adiabatic and diabatic processes to the vertical temperature profile will be explored in the upcoming sections. The height of the dendritic growth zone (DGZ) ranged from 650 to $500 \mathrm{hPa}$, consistent with the results of prior studies on winter precipitation at similar elevations (e.g. Auer and White 1982).

Analysis of modeled moisture profiles (Table 3.2) revealed moderate to high levels of water vapor (generally 2 to $3 \mathrm{~g} / \mathrm{kg}$ ) through much of the lower troposphere (i.e. below 700 $\mathrm{hPa}$ ). The maximum moisture contents ( 2.9 to $4.7 \mathrm{~g} / \mathrm{kg}$ ) were generally found at the levels associated with the WLZ. The decrease in moisture content seen below the inversion may be due to the advection of dry air from an upstream anticyclone. Moisture contents in the DGZ 
were between 1.6 and $2.2 \mathrm{~g} / \mathrm{kg}$, which is again consistent with observations from a larger sample of events (e.g. Auer and White 1982).

Analysis of modeled mean instantaneous vertical velocity profiles (Table 3.3) revealed strong upward motion, or lift, in the DGZ. Half of the profiles exhibited vertical velocities of at least $-10 \mathrm{~cm} / \mathrm{s}$ in the DGZ, with a maximum velocity of $-19.5 \mathrm{~cm} / \mathrm{s}$. Research conducted at several NWS offices in the eastern U.S. has shown that approximately 75 percent of events meeting Winter Storm Warning criteria had a mean instantaneous vertical velocity of at least $-10 \mathrm{~cm} / \mathrm{s}$ in the DGZ (J. Cortinas, personal communication, 2006). These velocities are three to four times greater than most synoptic-scale vertical velocities (Banacos and Ekster 2010) and are indicative of the strong dynamical lifting typically connected with heavy precipitation. In five of the eight profiles, the level of maximum lifting corresponded with the DGZ, resulting in enhanced snowfall rates (Auer and White 1982). In the other cases, the level of maximum lifting occurred above the DGZ, a situation that has been tied to higher snow-liquid ratios and greater overall snow accumulation (J. Cortinas, personal communication, 2006). 
Table 3.1. Modeled vertical temperature $\left({ }^{\circ} \mathrm{C}\right)$ profile for each snowstorm. Temperatures associated with the dendritic growth zone are highlighted in gray. The maximum temperature in the low-level inversion is underlined.

\begin{tabular}{|c|c|c|c|c|c|c|c|c|}
\hline $\begin{array}{l}\text { Pressure } \\
\quad(\mathrm{hPa})\end{array}$ & 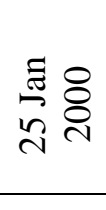 & 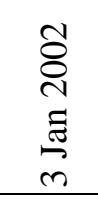 & $\begin{array}{l}\hat{0} \\
\text { 志 } \\
\text { స̃ }\end{array}$ & 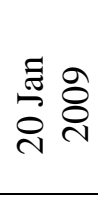 & $\sum_{\sim}^{\vec{z}} \delta$ & 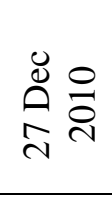 & 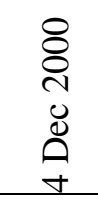 & 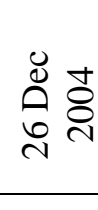 \\
\hline 300 & -45.1 & -46.4 & -48.4 & -42.6 & -44.9 & -44.9 & -41.4 & -43.2 \\
\hline 350 & -37.0 & -39.8 & -44.1 & -43.5 & -41.2 & -38.1 & -35.7 & -32.1 \\
\hline 400 & -28.5 & -32.5 & -36.5 & -40.7 & -38.7 & -32.1 & -30.4 & -26.5 \\
\hline 450 & -21.1 & -25.8 & -27.3 & -34.8 & -31.6 & -26.5 & -27.1 & -18.8 \\
\hline 500 & -16.7 & -20.3 & -23.2 & -29.7 & -26.8 & -20.3 & -24.8 & -16.1 \\
\hline 550 & -12.5 & -14.8 & -18.1 & -24.7 & -21.4 & -14.8 & -22.0 & -14.3 \\
\hline 600 & -8.3 & -11.0 & -14.3 & -19.9 & -18.1 & -10.7 & -18.2 & -9.5 \\
\hline 650 & -6.8 & -8.3 & -10.6 & -15.3 & -14.9 & -8.7 & -14.4 & -6.3 \\
\hline 700 & -3.0 & -6.0 & -7.0 & -12.4 & -12.8 & -7.1 & -9.8 & -3.9 \\
\hline 725 & -1.8 & -5.4 & -5.5 & -10.8 & -12.3 & -6.4 & -8.2 & -3.1 \\
\hline 750 & -1.2 & -4.8 & -4.1 & -9.2 & -12.2 & -5.8 & -6.4 & -2.5 \\
\hline 775 & -0.5 & -4.1 & -3.7 & -8.6 & -11.2 & -5.1 & -4.8 & -2.1 \\
\hline 800 & -0.4 & -3.2 & -2.9 & -7.8 & -10.0 & -4.8 & -3.4 & -2.4 \\
\hline 825 & -0.7 & -2.5 & -2.1 & -7.1 & -10.2 & -4.5 & -2.7 & -2.5 \\
\hline 850 & -1.6 & -2.4 & -1.6 & -7.0 & -8.4 & -4.6 & -2.1 & -2.8 \\
\hline 875 & -2.1 & -2.3 & -1.0 & -7.3 & -6.4 & -4.8 & -1.6 & -3.1 \\
\hline 900 & -3.1 & -3.1 & -1.2 & -7.2 & -4.9 & -4.6 & $\underline{-1.2}$ & -3.0 \\
\hline 925 & -3.4 & -3.7 & -2.0 & -6.3 & -3.1 & -4.1 & -1.4 & -2.4 \\
\hline 950 & -2.5 & -4.7 & -3.2 & -5.7 & -0.9 & -3.6 & -2.3 & -1.7 \\
\hline 975 & -1.2 & -4.2 & -3.2 & -3.8 & -0.5 & -2.5 & -2.4 & -1.7 \\
\hline $1000 / \mathrm{Sfc}$ & -0.7 & -2.9 & -2.5 & -2.2 & 0.2 & -0.9 & -1.8 & -1.1 \\
\hline
\end{tabular}


Table 3.2. Same as Table 3.1, except for the modeled vertical moisture profile $(\mathrm{g} / \mathrm{kg})$

\begin{tabular}{|c|c|c|c|c|c|c|c|c|}
\hline $\begin{array}{l}\text { Pressure } \\
(\mathrm{hPa})\end{array}$ & 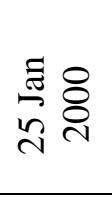 & 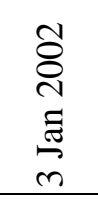 & 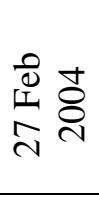 & 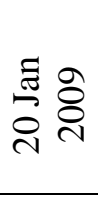 & 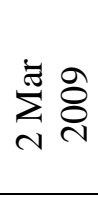 & 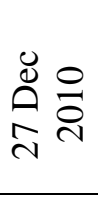 & 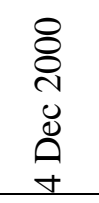 & 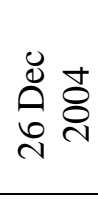 \\
\hline 300 & 0.16 & 0.10 & 0.08 & 0.03 & 0.04 & 0.05 & 0.05 & 0.19 \\
\hline 350 & 0.34 & 0.18 & 0.15 & 0.06 & 0.14 & 0.07 & 0.05 & 0.53 \\
\hline 400 & 0.74 & 0.31 & 0.31 & 0.17 & 0.22 & 0.16 & 0.06 & 0.86 \\
\hline 450 & 1.42 & 0.67 & 0.69 & 0.39 & 0.48 & 0.69 & 0.15 & 1.52 \\
\hline 500 & 2.01 & 1.22 & 0.97 & 0.60 & 0.76 & 1.36 & 0.29 & 1.85 \\
\hline 550 & 2.65 & 1.96 & 1.42 & 0.86 & 1.19 & 2.12 & 0.63 & 2.22 \\
\hline 600 & 3.22 & 2.56 & 2.06 & 1.26 & 1.52 & 2.49 & 1.12 & 2.95 \\
\hline 650 & 3.40 & 3.03 & 2.49 & 1.69 & 1.84 & 2.71 & 1.63 & 3.60 \\
\hline 700 & 3.90 & 3.38 & 3.18 & 2.06 & 2.02 & 2.88 & 2.36 & 4.06 \\
\hline 725 & 4.26 & 3.44 & 3.51 & 2.27 & 2.04 & 2.98 & 2.69 & 4.12 \\
\hline 750 & 4.48 & 3.46 & 3.80 & 2.46 & 1.97 & 3.03 & 3.00 & 4.08 \\
\hline 775 & 4.67 & 3.55 & 3.84 & 2.49 & 1.93 & 3.10 & 3.33 & $\underline{3.88}$ \\
\hline 800 & $\underline{4.61}$ & 3.69 & 3.92 & 2.54 & $\underline{1.98}$ & 3.12 & 3.60 & 3.50 \\
\hline 825 & 4.37 & 3.80 & 3.95 & 2.57 & 2.11 & $\underline{3.10}$ & 3.69 & 3.38 \\
\hline 850 & 3.97 & 3.76 & 3.97 & 2.47 & 2.33 & 3.07 & 3.77 & 3.09 \\
\hline 875 & 3.65 & $\underline{3.66}$ & $\underline{3.98}$ & 2.20 & 2.66 & 2.95 & 3.77 & 2.97 \\
\hline 900 & 3.00 & 3.28 & 3.74 & 2.04 & 2.91 & 2.93 & $\underline{3.32}$ & 2.98 \\
\hline 925 & 2.78 & 3.08 & 3.40 & 2.14 & 3.17 & 2.90 & 3.07 & 3.13 \\
\hline 950 & 2.93 & 2.68 & 3.00 & 2.26 & 3.58 & 2.83 & 2.55 & 3.33 \\
\hline 975 & 3.24 & 2.67 & 2.89 & 2.67 & 3.76 & 3.00 & 2.38 & 3.49 \\
\hline $1000 / \mathrm{Sfc}$ & 3.31 & 2.87 & 3.03 & 2.92 & 3.76 & 3.27 & 2.36 & 3.22 \\
\hline
\end{tabular}


Table 3.3. Same as Table 3.1, except for the modeled mean instantaneous vertical velocity $(\mathrm{cm} / \mathrm{s})$. Negative (positive) values indicate upward (downward) vertical motion.

\begin{tabular}{|c|c|c|c|c|c|c|c|c|}
\hline $\begin{array}{l}\text { Pressure } \\
\quad(\mathrm{hPa})\end{array}$ & 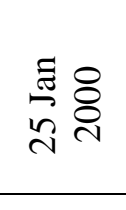 & 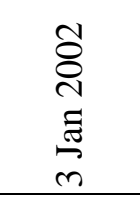 & 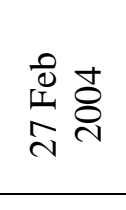 & సี & $\sum_{\sim}^{\pi} \underset{\delta}{\delta}$ & 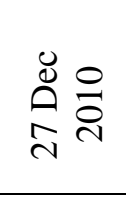 & 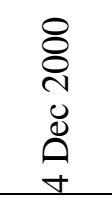 & 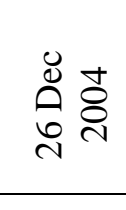 \\
\hline 300 & -12.67 & -1.90 & 0.72 & -1.88 & -3.11 & -3.68 & 6.64 & -11.38 \\
\hline 350 & -20.72 & -1.50 & -10.00 & -2.29 & -5.25 & -6.95 & 7.26 & -12.29 \\
\hline 400 & -21.36 & -1.72 & -15.94 & -2.69 & -5.45 & -8.08 & 6.11 & -10.29 \\
\hline 450 & -21.07 & -3.22 & -9.21 & -2.99 & -6.48 & -7.00 & -1.24 & -9.61 \\
\hline 500 & -19.49 & -6.45 & -8.06 & -3.15 & -9.55 & -10.50 & -6.08 & -9.71 \\
\hline 550 & -17.69 & -10.02 & -8.58 & -5.78 & -7.31 & -13.63 & -7.26 & -12.31 \\
\hline 600 & -14.73 & -9.73 & -8.99 & -5.82 & -7.66 & -10.34 & -6.69 & -8.45 \\
\hline 650 & -13.50 & -8.67 & -8.57 & -5.24 & -10.27 & -7.50 & -7.66 & -4.14 \\
\hline 700 & -10.36 & -7.39 & -6.31 & -5.19 & -10.06 & -3.93 & -7.23 & -1.50 \\
\hline 725 & -9.22 & -6.56 & -5.18 & -5.02 & -8.09 & -1.96 & -7.00 & 0.48 \\
\hline 750 & -8.58 & -5.07 & -3.93 & -4.97 & -6.86 & -0.68 & -6.70 & 2.26 \\
\hline 775 & -6.85 & -3.50 & -3.76 & -4.97 & -5.97 & -0.20 & -6.33 & $\underline{3.79}$ \\
\hline 800 & -4.95 & -1.56 & -3.92 & -4.58 & -4.29 & 0.28 & -5.14 & 3.34 \\
\hline 825 & -3.22 & -0.39 & -2.67 & -3.94 & -3.32 & $\underline{0.64}$ & -3.69 & 3.50 \\
\hline 850 & -1.68 & 0.56 & -1.47 & -3.16 & -2.57 & 0.49 & -2.07 & 3.94 \\
\hline 875 & -1.07 & $\underline{1.57}$ & $\underline{-0.86}$ & -1.27 & -1.89 & 0.09 & -0.56 & 3.72 \\
\hline 900 & -0.50 & 1.82 & -0.01 & -0.31 & -1.34 & 0.13 & $\underline{0.59}$ & 3.47 \\
\hline 925 & -0.36 & 1.92 & 1.33 & 0.00 & -0.31 & 0.23 & 0.77 & 2.97 \\
\hline 950 & -0.46 & 1.71 & 0.50 & 0.05 & -0.45 & 0.14 & 0.24 & 2.60 \\
\hline 975 & -0.96 & 1.03 & 0.71 & -0.21 & -1.07 & 0.01 & -0.30 & 1.99 \\
\hline $1000 / \mathrm{Sfc}$ & -0.24 & 0.14 & 0.36 & -0.16 & -0.21 & -0.25 & -0.42 & 0.48 \\
\hline
\end{tabular}




\subsection{Trajectory Analyses}

\subsubsection{Composite Trajectories}

Figure 3.1 illustrates the mean horizontal component of the 72-hr back trajectories associated with the eight snowstorms. These trajectories were calculated backward in time from three critical vertical pressure levels at the time and location of heaviest precipitation. Each 72-hr back trajectory was decomposed into 1-hr segments. These segments correspond to the individual symbols that trace each trajectory in Figure 3.1. Composite trajectories were used due to the similarities among the individual storms and to facilitate comparisons between the different vertical levels.

The near-surface trajectory originated over Lake Huron and exhibited an anticyclonic curvature as it approached central North Carolina. In contrast, air parcels terminating in the WLZ originated over Lake Superior. These WLZ parcels traveled southeastward to a point off of the mid-Atlantic coast before shifting course to the southwest towards central North Carolina. While the near-surface and WLZ trajectories were similar across all the snowstorms, examination of the DGZ trajectories revealed two distinct paths and source regions. In four of the eight snowstorms, the trajectory terminating in the DGZ originated east of Lake Huron and traveled anticyclonically over the Northeast U.S. (hereafter referred to as the "DGZ-anticyclonic" trajectory; Figure 3.1). These air parcels continued traveling southward over the Atlantic Ocean before re-curving toward central North Carolina. In the other four snowstorms, the air associated with the DGZ originated over the Caribbean Sea and exhibited a subtle cyclonic curvature as it approached central North Carolina (hereafter referred to as the "DGZ-cyclonic" trajectory; Figure 3.1). 
The corresponding mean vertical component of the 72-hr back trajectories is illustrated in Figure 3.2. As in Figure 3.1, each trajectory was decomposed into 1-hr segments. The near-surface air parcel originated at approximately $1 \mathrm{~km}$ above ground level and gradually descended over the course of the trajectory. The WLZ trajectory originated at approximately $2.5 \mathrm{~km}$ and descended to near $1 \mathrm{~km}$ near the Delmarva Peninsula (Figure 3.1), with a subtle rise in altitude over the last 6 hours of the trajectory. Both DGZ trajectories originated between 1 and $1.5 \mathrm{~km}$ and descended to below $1 \mathrm{~km}$ at T-38 hours (Figure 3.2). The DGZ-cyclonic trajectory reached a minimum height of approximately $0.5 \mathrm{~km}$ at T-30 hours while the DGZ-anticyclonic trajectory remained just below $1 \mathrm{~km}$. At T-12 hours, both DGZ trajectories rose abruptly from near $1 \mathrm{~km}$ to their terminating altitude near $5 \mathrm{~km}$, reaffirming the presence of strong upward vertical motion revealed earlier in the modeled vertical profile.

Figure 3.3 illustrates the air temperature along each trajectory. The near-surface air parcels exhibited a gradual warming from -12 to $-3^{\circ} \mathrm{C}$ over the course of the trajectory. This near-surface trajectory traces the southward progression of cold air generally associated with a surface anticyclone moving out of central Canada. The WLZ trajectory revealed a warming of air parcels from -17 to $0^{\circ} \mathrm{C}$ over the first half of the trajectory, followed by a gradual cooling of air parcels to $-5^{\circ} \mathrm{C}$ as they entered central North Carolina. Air parcels associated with the DGZ-anticyclonic trajectory warmed from -7 to $5^{\circ} \mathrm{C}$ over the first twothirds of the trajectory, and then abruptly cooled to the DGZ temperature due to adiabatic expansion resulting from strong upward vertical motion. In contrast, air parcels associated with the DGZ-cyclonic trajectory remained remarkably warm (near $15^{\circ} \mathrm{C}$ ) for most of the trajectory before abruptly cooling as they entered the DGZ. 
Moisture composites were calculated along each trajectory at each critical vertical pressure level (Figure 3.4a). The moisture content of the near-surface air parcels increased slightly throughout most of the trajectory period ( 1 to $2 \mathrm{~g} / \mathrm{kg}$ ), and then increased from 2 to 3 $\mathrm{g} / \mathrm{kg}$ over the last 6 hours of the trajectory. This short-term increase may be due to virga or evaporating precipitation. The moisture content of the WLZ air parcels exhibited a gradual increase from 1 to $3 \mathrm{~g} / \mathrm{kg}$ over the first half of the trajectory, and then exhibited little change over the remainder of the trajectory. In contrast, the moisture content of the DGZ trajectories revealed well-defined evaporation-precipitation cycles. These cycles are identified by the initial rise in moisture content resulting from surface evaporative fluxes (e.g. from an upstream body of water) followed by a decrease in moisture resulting from condensation and deposition (James et al. 2004). Figure $3.4 b$ provides a schematic illustration of a typical evaporation-precipitation cycle. Though the rate increase in moisture content over the first part of the cycle (T-72 to T-12 hours) is similar between the DGZ trajectory types, the DGZcyclonic trajectory exhibited much larger overall moisture contents $(4.5$ to $8.2 \mathrm{~g} / \mathrm{kg}$ ) than the DGZ-anticyclonic trajectory ( 1.8 to $5.8 \mathrm{~g} / \mathrm{kg})$. Moreover, the rate decrease in moisture content (i.e. the rate of condensation and deposition) was much greater with DGZ-cyclonic trajectories. The reduction in moisture resulting from condensation and deposition in the presence of strong upward vertical velocity indicates that much of the moisture supply was converted into precipitation (James et al. 2004).

To determine whether diabatic processes were occurring along each trajectory, the potential temperature $(\theta)$ of the air parcels was calculated. Figure 3.5 illustrates the composite $\theta$ values along the trajectories from each critical vertical pressure level. The $\theta$ values along both the near-surface and WLZ trajectories were nearly constant over the 72-hr 
trajectory period. In contrast, the DGZ-anticyclonic and DGZ-cyclonic trajectories exhibited increases in $\theta$ over the final 10 hours of the trajectory from 277 to $295 \mathrm{~K}$ and from 294 to 308 $\mathrm{K}$, respectively. The increase in $\theta$ corresponded with large reductions in moisture content along the DGZ trajectories (Figure 3.4a). It is likely that the latent heat released due to condensation and deposition resulted in strong diabatic heating, which is reflected by the increase in $\theta$ along the trajectories. Therefore, the increase in $\theta$ suggests a diabatic contribution to the DGZ trajectories from condensation and deposition.

Figure 3.6 shows the net diabatic contribution to air parcel temperatures along each trajectory. Over the 72-hr trajectory period, diabatic processes contributed on average between 9 and $13^{\circ} \mathrm{C}$ of warming to the DGZ trajectories. In other words, in the absence of diabatic processes, the resulting air parcel temperature by the end of the trajectory would have been 9 to $13^{\circ} \mathrm{C}$ cooler than observed at the beginning of the trajectory. By comparison, diabatic processes contributed less than $4^{\circ} \mathrm{C}$ of warming to the near-surface trajectories. Interestingly, several snowstorms exhibited net diabatic cooling $\left(<0^{\circ} \mathrm{C}\right)$ of air parcels along the WLZ trajectory. This was likely due to latent heat absorption from evaporation, as moisture contents steadily increased along the trajectory (Figure 3.4a).

Because the WLZ temperature is frequently used to discriminate between snow and freezing rain/sleet scenarios, it is important to diagnose the processes that contribute to the temperature at this level. Figure 3.7 illustrates the observed air parcel temperature and the adiabatic air parcel temperature (i.e. the temperature of the air parcel with no diabatic contributions) at 6-hr segments along the trajectory. For the first half of the trajectory the observed temperature was slightly higher on average than the adiabatic temperature, which indicates the occurrence of diabatic warming. In contrast, the observed temperature over the 
last half of the trajectory was slightly lower than the adiabatic temperature. In particular, the range in adiabatic temperature among the eight snowstorms over the final 6 hours of the trajectory was -7 to $3^{\circ} \mathrm{C}$. This indicates that in some cases the observed temperature would have been above freezing at the time of precipitation in the absence of diabatic cooling. With a WLZ temperature of $3^{\circ} \mathrm{C}$ it is conceivable that at least partial melting of precipitation would have occurred, resulting in either wet snow, sleet, or freezing rain. These results indicate the importance of accurately diagnosing the amount of diabatic cooling due to latent heat absorption from evaporation when making a precipitation-type forecast in the region. 


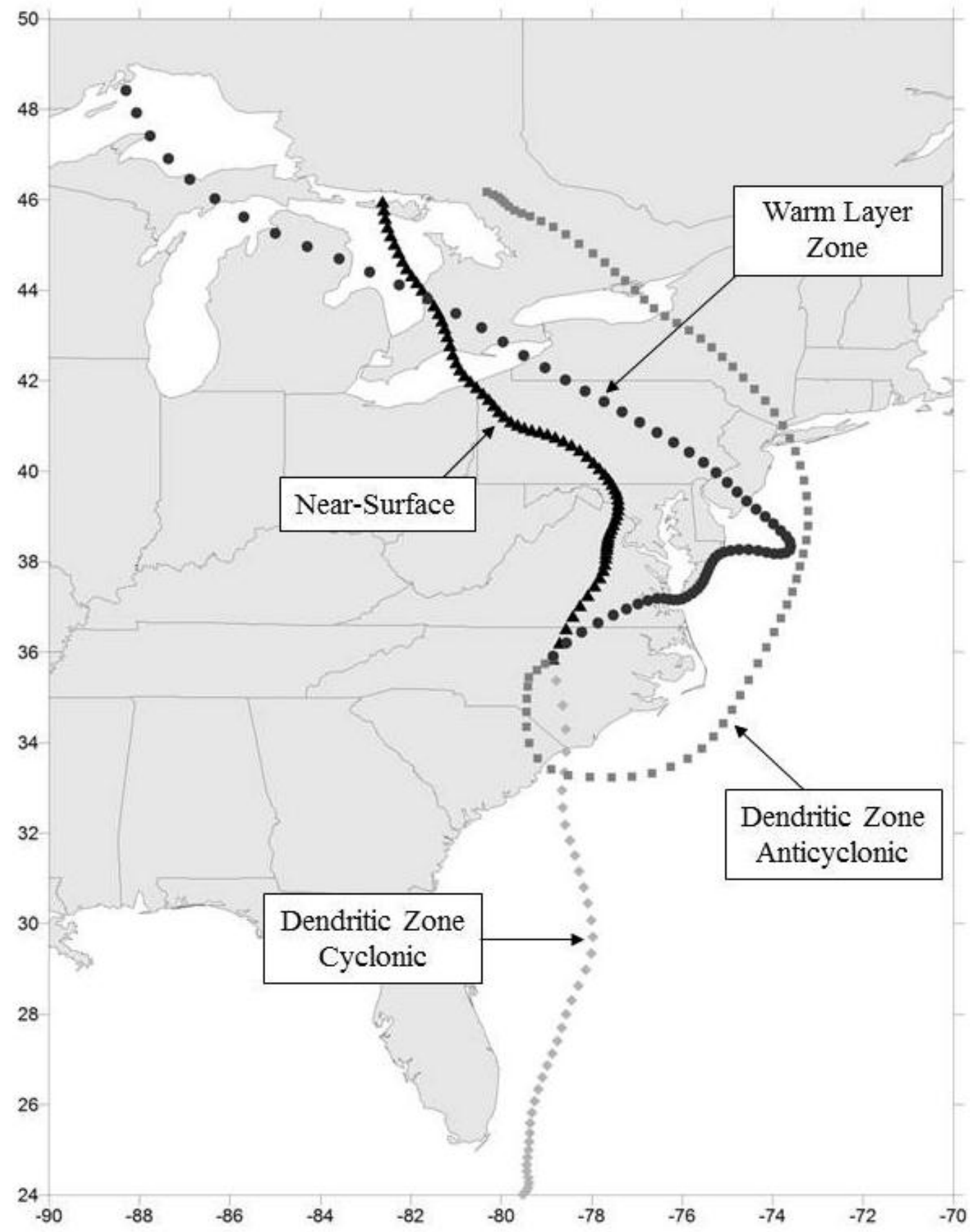

Figure 3.1. Horizontal trajectory composites of air parcels terminating at the near-surface, warm layer zone, and dendritic growth zone in the region of heavy snowfall. 


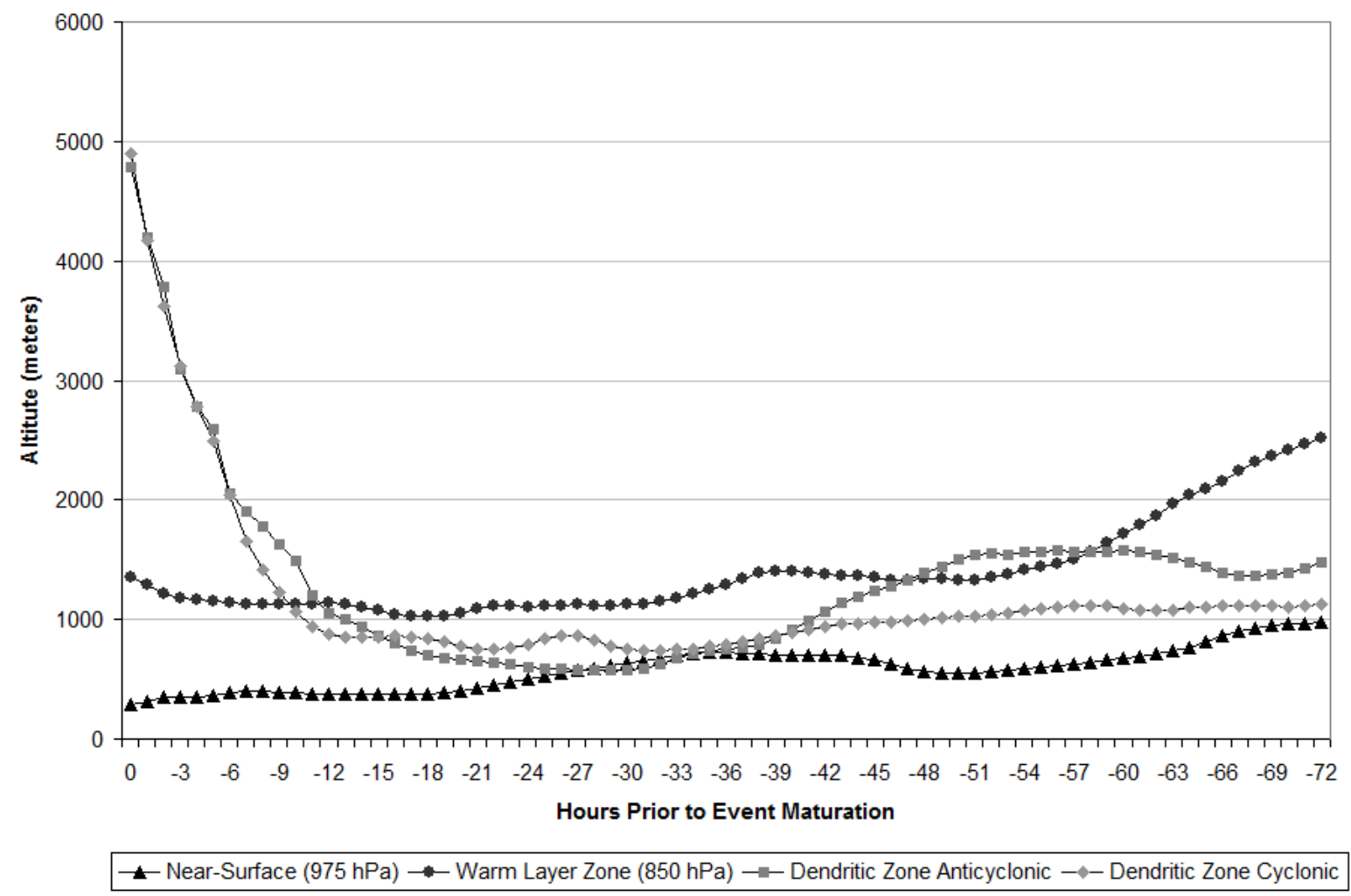

Figure 3.2. Vertical trajectory composites of air parcels terminating at the near-surface, warm layer zone, and dendritic growth zone in the region of heavy snowfall. Composites with range bars can be found in the appendix. 


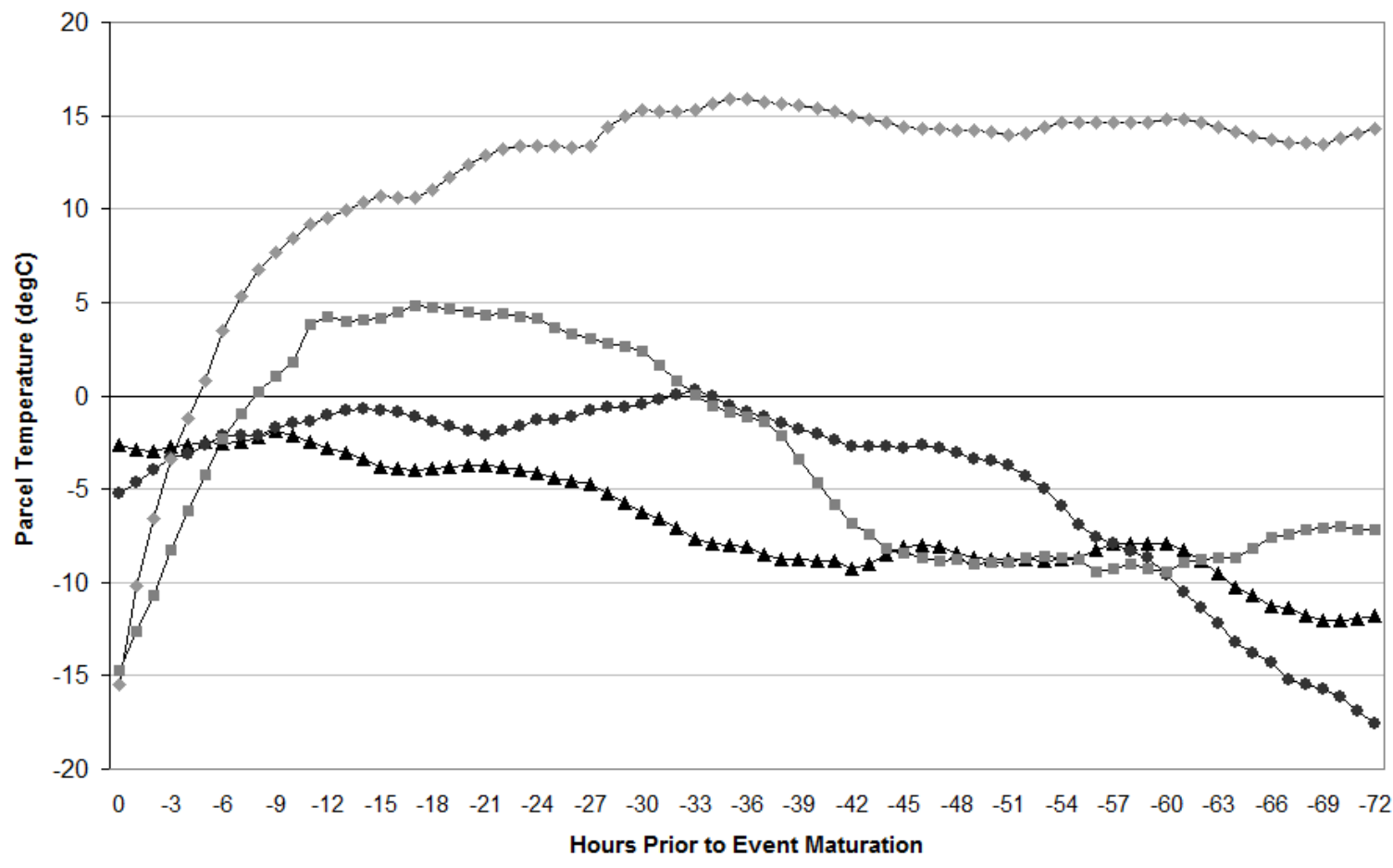

$\downarrow$ Near-Surface $(975 \mathrm{hPa}) \rightarrow-$ Warm Layer Zone $(850 \mathrm{hPa}) \multimap-$ Dendritic Zone Anticyclonic — - Dendritic Zone Cyclonic

Figure 3.3. Same as Figure 3.2, except for composite trajectories of air parcel temperature. 


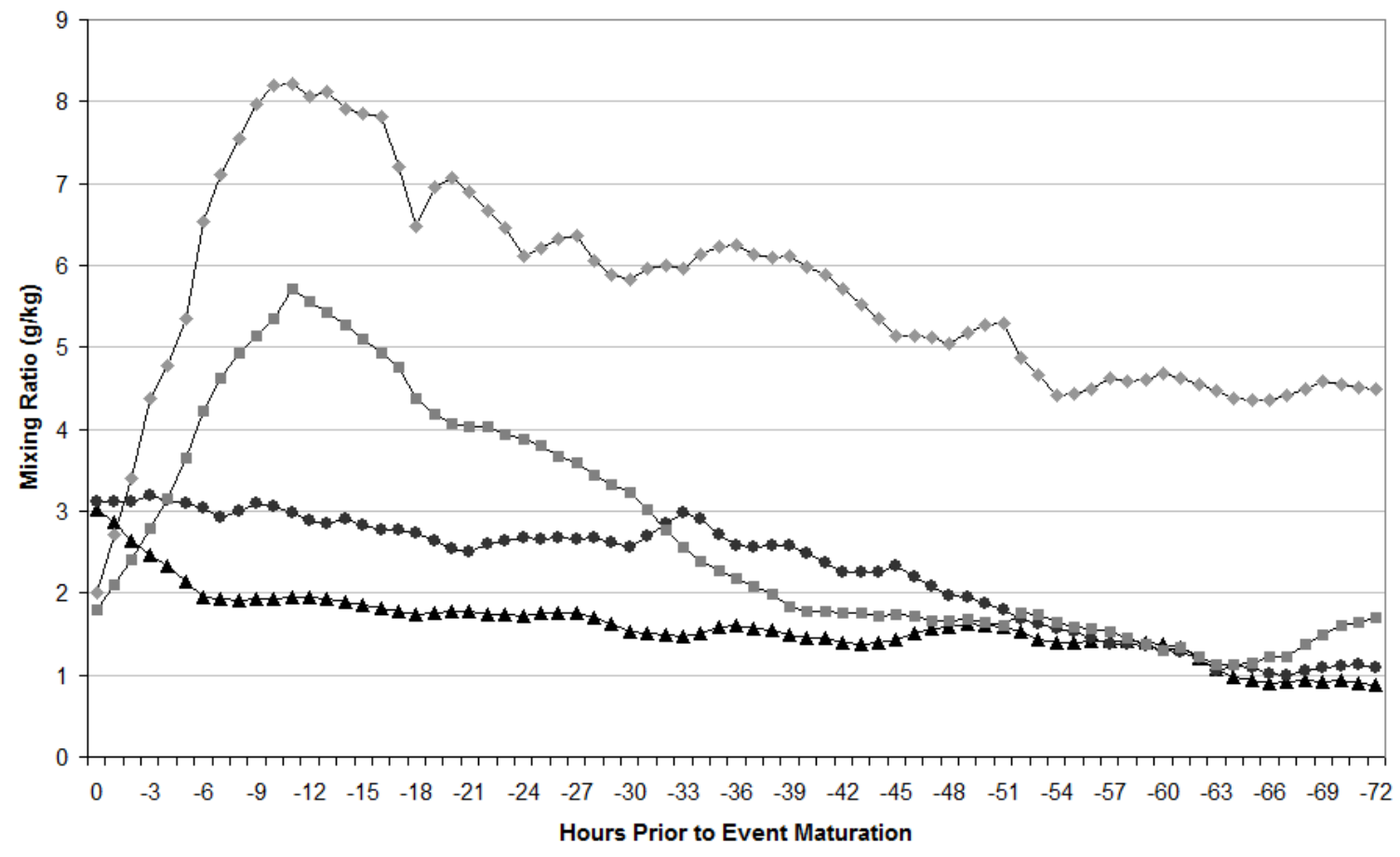

— Near-Surface $(975 \mathrm{hPa}) \rightarrow-$ Warm Layer Zone $(850 \mathrm{hPa}) \longrightarrow$ - Dendritic Zone Anticyclonic - - Dendritic Zone Cyclonic

(A)

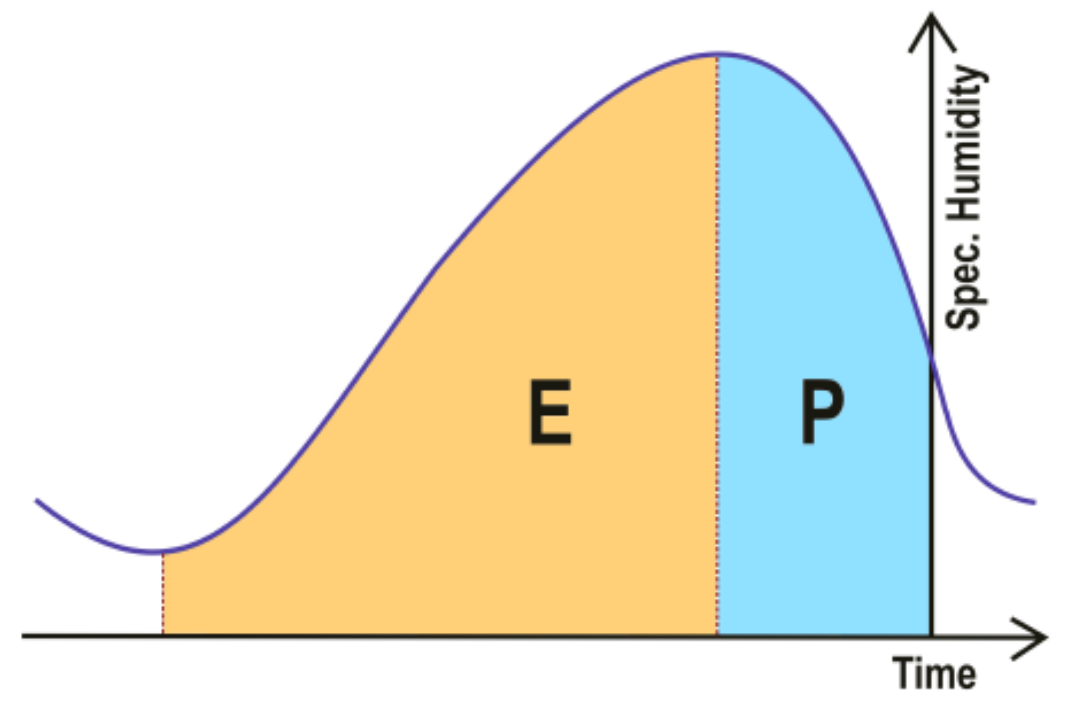

(B)

Figure 3.4. (A) Same as Figure 3.2, except for moisture composites along each trajectory. (B) Schematic illustration of the moisture curve representing a typical evaporationprecipitation cycle where " $\mathrm{E}$ " is the primary period of evaporative increase in humidity leading up to the final period of precipitative loss "P" of humidity. From James et al. (2004), their Figure 10. 


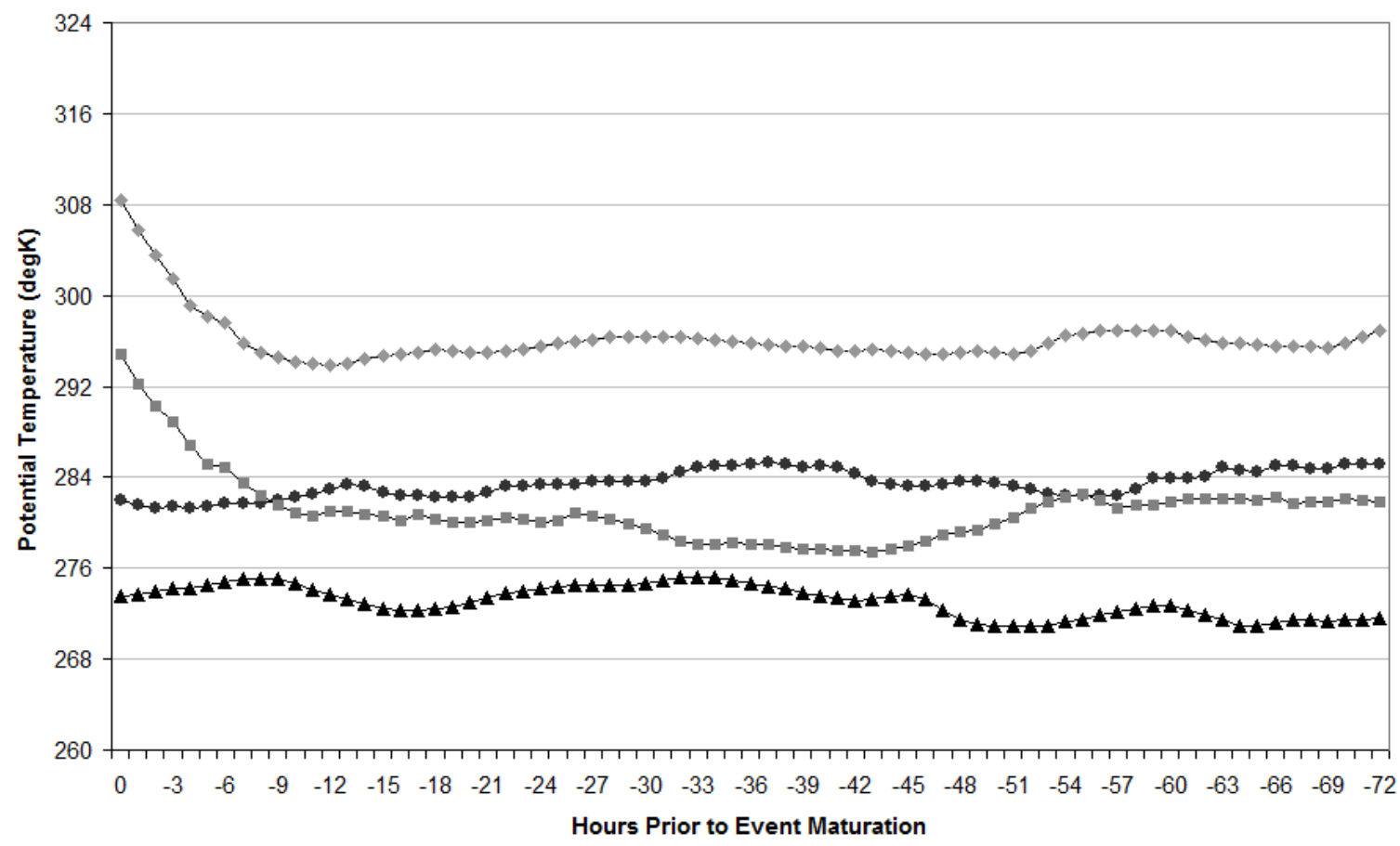

- Near-Surface $(975 \mathrm{hPa}) \rightarrow$ - Warm Layer Zone $(850 \mathrm{hPa}) \longrightarrow$ - Dendritic Zone Anticyclonic — - Dendritic Zone Cyclonic

Figure 3.5. Same as Figure 3.2, except for composite trajectories of air parcel potential temperature. 


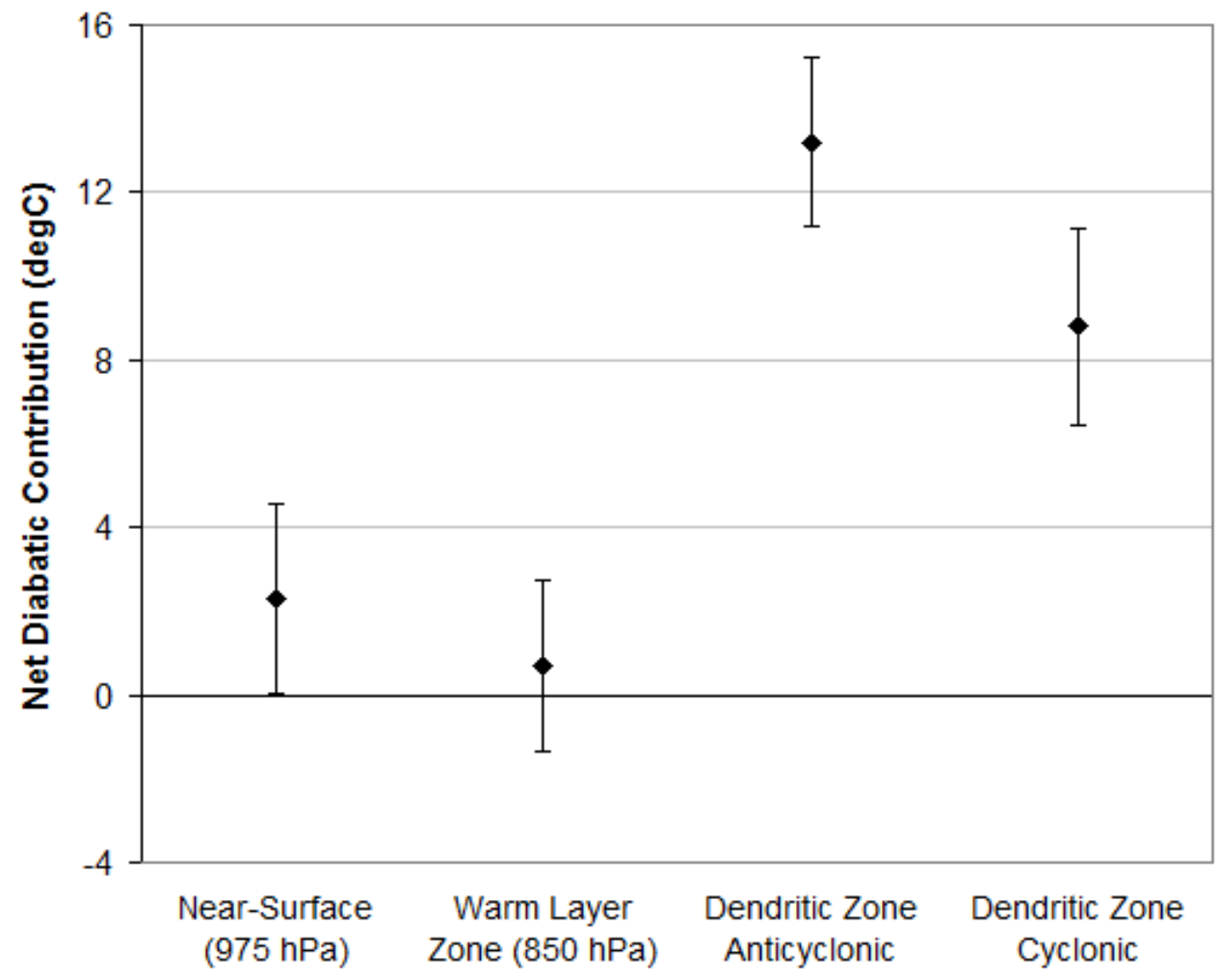

Figure 3.6. Net diabatic contribution to air parcel temperature at each critical level in the region of heavy snowfall. Range bars indicate the maximum and minimum values among all eight snowstorms. 


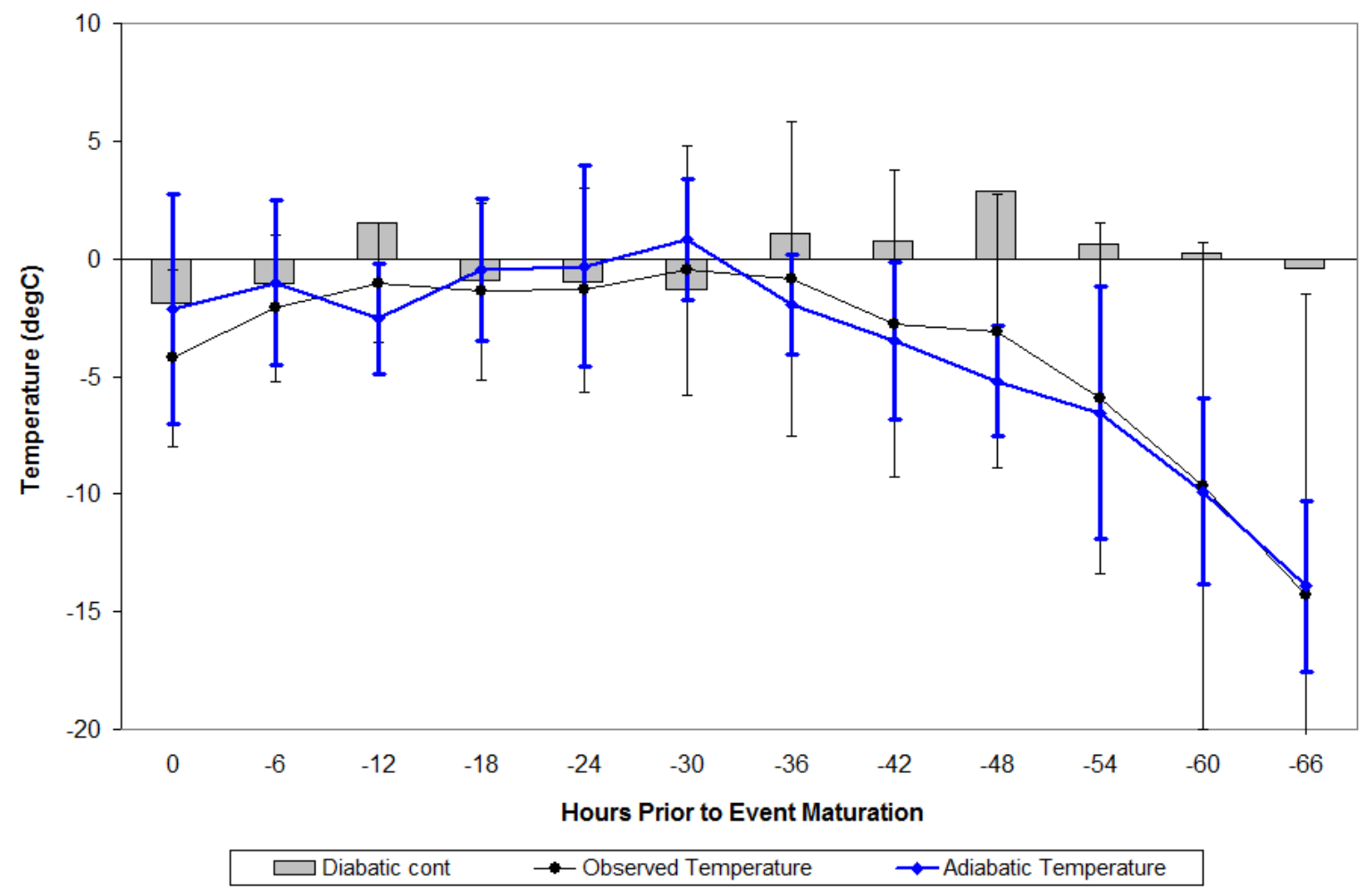

Figure 3.7. Warm layer zone trajectories of observed air parcel temperature (black line), adiabatic temperature (thick blue line), and diabatic contribution (gray bars) in the region of heavy snowfall at 6-hr trajectory segments. Range bars indicate the maximum and minimum values among all eight snowstorms. 


\subsubsection{Comparative and Ensemble Trajectories}

To distinguish between trajectories at locations and times with different precipitation types and intensities in a given storm, a set of comparative trajectories was calculated for two of the eight snowstorms. In addition, a set of ensemble trajectories was calculated for the same two snowstorms to assess how variations in the space-time character and source regions of the trajectories, due to uncertainties in the trajectory calculations, affect the meteorological properties of the air advected into the region of heavy precipitation.

\subsection{2a. Case I: 24-25 January 2000}

The snowstorm of 24-25 January 2000 was one of the most remarkable winter storms ever recorded across central North Carolina, both for the record snowfall amounts that were recorded and for the failure of most numerical weather prediction models to adequately forecast the evolution of the storm and the resulting precipitation (Brennan and Lackmann 2005). It was selected for further analysis because of the great disparity in snowfall totals and precipitation rates across the study area. Snowfall amounts across the Raleigh CWA ranged from approximately 2 in. $(5.1 \mathrm{~cm})$ in the western and eastern-most areas of the CWA to as much as 22 in. $(55.9 \mathrm{~cm}$ ) across the central portion of the CWA (see appendix Figure A1). Raleigh-Durham, NC (RDU) recorded 20.3 in. $(51.6 \mathrm{~cm})$, which is the most snowfall from a single storm in a station record extending back to 1944. By comparison, WinstonSalem, NC (INT), located $130 \mathrm{~km}$ west of RDU (see Figure 2.1), recorded only 2.3 in. (5.8 $\mathrm{cm}$ ) of snowfall from the event, while Rocky Mount, NC, located $94 \mathrm{~km}$ east of RDU, recorded $6.8 \mathrm{in} .(17.3 \mathrm{~cm})$ of snow. The disparity in snowfall totals across the CWA is further revealed in the hourly precipitation totals from the storm (Table 3.4). For example, 
RDU recorded 5 in. (12.7 cm) of snow in one hour (06 UTC 25 January), more than double the amount of precipitation observed at RWI, while INT recorded just a trace of precipitation. Examination of the corresponding back trajectories provides some insight into the disparity in the precipitation totals. Of particular importance are the differences in the spacetime character and meteorological properties of the DGZ trajectories. At RDU and RWI, the DGZ trajectories originated near the Caribbean Sea and traveled northward over the Gulf Stream, then turned cyclonically and ascended into the region of precipitation (Figure 3.8a,b). These trajectories spent considerable time over warm ocean waters and revealed well-defined evaporation-precipitation cycles and high fluxes of latent heat (Figure 3.9). However, the maximum moisture content of the DGZ air at RDU was nearly $4 \mathrm{~g} / \mathrm{kg}$ greater than at RWI, indicating that there was more moisture available for precipitation at RDU compared to RWI.

In contrast, the DGZ trajectory at INT originated near the southern tip of Florida and turned anticyclonically across northern Florida and Georgia before turning cyclonically up the Carolina coastline and into the region of precipitation (Figure 3.8c). The moisture content along the INT trajectory exhibited a gradual decline over the last half of the trajectory, with only half the moisture available for precipitation over the last 6 hours of the trajectory compared to RDU and RWI (Figure 3.9). The reduction in moisture and latent heat fluxes seen in the INT trajectory coincided with the air parcel moving out from over the Gulf of Mexico and crossing into southern Georgia. Moreover, over the final 6 hours of the DGZ trajectories, the air parcels terminating at INT ascended at a slower rate than at RWI and RDU. The mean vertical velocity was $-17 \mathrm{~cm} / \mathrm{s}$ at RDU, versus $-13 \mathrm{~cm} / \mathrm{s}$ at RWI and -9 $\mathrm{cm} / \mathrm{s}$ at INT. The results of the trajectory calculations therefore suggest that a combination of 
greater moisture content due to increased latent heat fluxes and greater upward vertical velocity contributed to higher precipitation rates at RDU.

The technique of Draxler (2003) was used to calculate a set of ensemble trajectories from RDU for the $24-25$ January 2000 snowstorm. This technique tests the sensitivity of the back trajectories to changes in the horizontal and vertical offset of the meteorological input data. Figure 3.10 illustrates the space-time character of the near-surface trajectory ensemble members. Most of the trajectories originated over the mid-Atlantic and Midwest regions and exhibited very little vertical motion. Four of the 27 ensemble members originated across Montana and Wyoming and descended nearly $2 \mathrm{~km}$ over the 72-hr trajectory period. These sinking motions contributed to adiabatic warming of the air parcels from approximately -20 to $1^{\circ} \mathrm{C}$, while the other ensemble members exhibited below freezing temperatures throughout the trajectory period (Figure 3.11). In the cases where the near-surface temperature was above freezing, the model likely failed to resolve the slow movement of the surface anticyclone and therefore underestimated the duration and magnitude of low-level cold air advection (i.e. a warm bias).

The set of ensemble trajectories terminating in the WLZ is illustrated in Figure 3.12. The trajectories diverged with time, with two preferred source regions emerging after 72 hours: one over the Great Lakes, corresponding to the composite trajectory in Figure 3.1, and one over the northwestern U.S. Four of the 27 ensemble members, each originating over the Great Lakes, exhibited above freezing WLZ temperatures at T-0 hours (Figure 3.13). These trajectories gradually descended over time and warmed from approximately -25 to $2^{\circ} \mathrm{C}$. As in the composite analysis, the net diabatic contribution to air parcel temperature was calculated for all WLZ ensemble members with comparisons between parcels that were 
above freezing and those that were below freezing. Figure 3.14 reveals that over the last 12 hours of the trajectories, ensemble members with above freezing WLZ temperatures exhibited on average an additional $17^{\circ} \mathrm{C}$ of warming due to diabatic processes. In contrast, ensemble members with below freezing WLZ temperature exhibited only $3^{\circ} \mathrm{C}$ of warming from diabatic processes. Thus, diabatic processes may have been a key contributor to temperature in the WLZ.

Figure 3.15 illustrates the set of ensemble trajectories terminating in the DGZ. Seventeen of the 27 ensemble members resembled the composite DGZ-cyclonic trajectory (see Figure 3.1). In contrast, the remaining ensemble members spent considerable time over the Gulf of Mexico before turning cyclonically along the Southeast coast. These trajectory paths were also distinguished by their meteorological properties. In particular, the mean vertical velocity over the final 6 hours of the trajectory was $-13 \mathrm{~cm} / \mathrm{s}$ (range of -11.8 to -13.9 $\mathrm{cm} / \mathrm{s}$ ) for Atlantic trajectories and $-10 \mathrm{~cm} / \mathrm{s}$ (range of -7.8 to $-12.6 \mathrm{~cm} / \mathrm{s}$ ) for Gulf of Mexico trajectories. In addition, the decrease in moisture content over the final 12 hours of the trajectory was much greater for the Atlantic trajectories (Figure 3.16). These results suggest that the rate of precipitation formation was greater in association with the Atlantic trajectories. 
Table 3.4. Hourly snowfall totals (in liquid equivalent) for the 24-25 January 2000 snowstorm at RDU, INT, and RWI. Values in brackets indicate periods of rain. Back trajectories were computed from the time highlighted in boldface.

\begin{tabular}{|c|c|c|c|c|}
\hline Date & $\begin{array}{c}\text { Time } \\
\text { (UTC) }\end{array}$ & RDU & INT & RWI \\
\hline $01 / 24 / 2000$ & 23 & [Tr] & 0.00 & 0.00 \\
\hline $01 / 25 / 2000$ & 00 & {$[0.02]$} & 0.00 & {$[\mathrm{Tr}]$} \\
\hline $01 / 25 / 2000$ & 01 & 0.04 & 0.00 & {$[0.03]$} \\
\hline $01 / 25 / 2000$ & 02 & 0.06 & 0.00 & {$[0.06]$} \\
\hline $01 / 25 / 2000$ & 03 & 0.06 & 0.00 & 0.03 \\
\hline $01 / 25 / 2000$ & 04 & 0.05 & 0.00 & 0.03 \\
\hline $01 / 25 / 2000$ & 05 & 0.06 & $\operatorname{Tr}$ & 0.05 \\
\hline $01 / 25 / 2000$ & 06 & 0.15 & $\operatorname{Tr}$ & 0.06 \\
\hline $01 / 25 / 2000$ & 07 & 0.10 & 0.01 & 0.03 \\
\hline $01 / 25 / 2000$ & 08 & 0.08 & 0.02 & 0.02 \\
\hline $01 / 25 / 2000$ & 09 & 0.08 & 0.02 & $\operatorname{Tr}$ \\
\hline $01 / 25 / 2000$ & 10 & 0.06 & 0.02 & $\operatorname{Tr}$ \\
\hline $01 / 25 / 2000$ & 11 & $\operatorname{Tr}$ & 0.01 & 0.02 \\
\hline $01 / 25 / 2000$ & 12 & $\operatorname{Tr}$ & 0.02 & 0.02 \\
\hline $01 / 25 / 2000$ & 13 & 0.02 & 0.03 & $\operatorname{Tr}$ \\
\hline $01 / 25 / 2000$ & 14 & $\operatorname{Tr}$ & 0.01 & $\operatorname{Tr}$ \\
\hline $01 / 25 / 2000$ & 15 & 0.01 & 0.00 & $\operatorname{Tr}$ \\
\hline $01 / 25 / 2000$ & 16 & $\operatorname{Tr}$ & 0.00 & $\operatorname{Tr}$ \\
\hline $01 / 25 / 2000$ & 17 & $\operatorname{Tr}$ & 0.00 & $\operatorname{Tr}$ \\
\hline $01 / 25 / 2000$ & 18 & $\operatorname{Tr}$ & 0.00 & $\operatorname{Tr}$ \\
\hline $01 / 25 / 2000$ & 19 & 0.01 & 0.00 & 0.00 \\
\hline
\end{tabular}



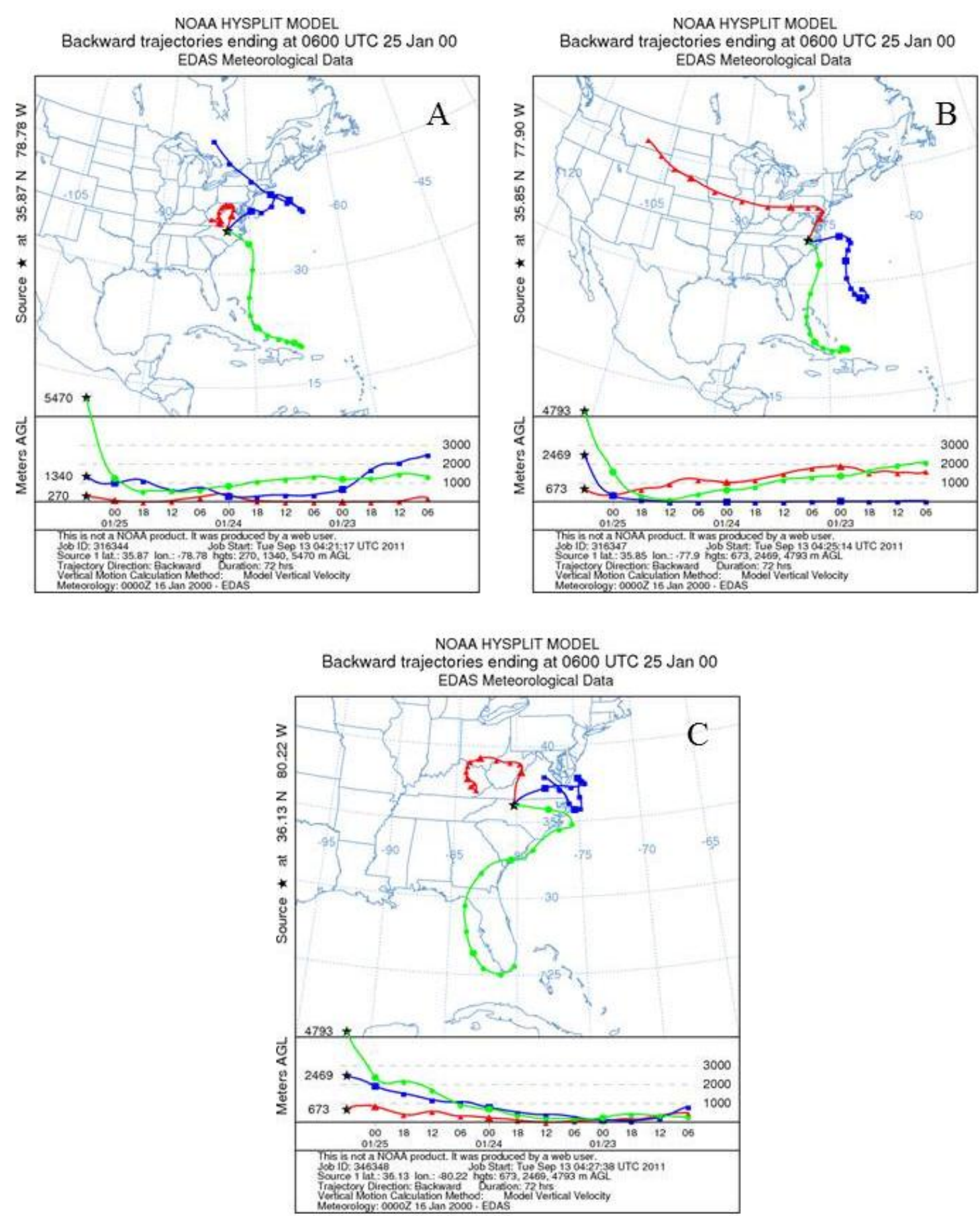

Figure 3.8. Horizontal and vertical trajectories for 06 UTC 25 January 2000 terminating at the near-surface level (red trajectories), WLZ (blue trajectories), and DGZ (green trajectories) at (A) Raleigh-Durham (RDU), (B), Rocky Mount (RWI), and (C) WinstonSalem (INT). Vertical trajectories are shown at the bottom of each panel. 

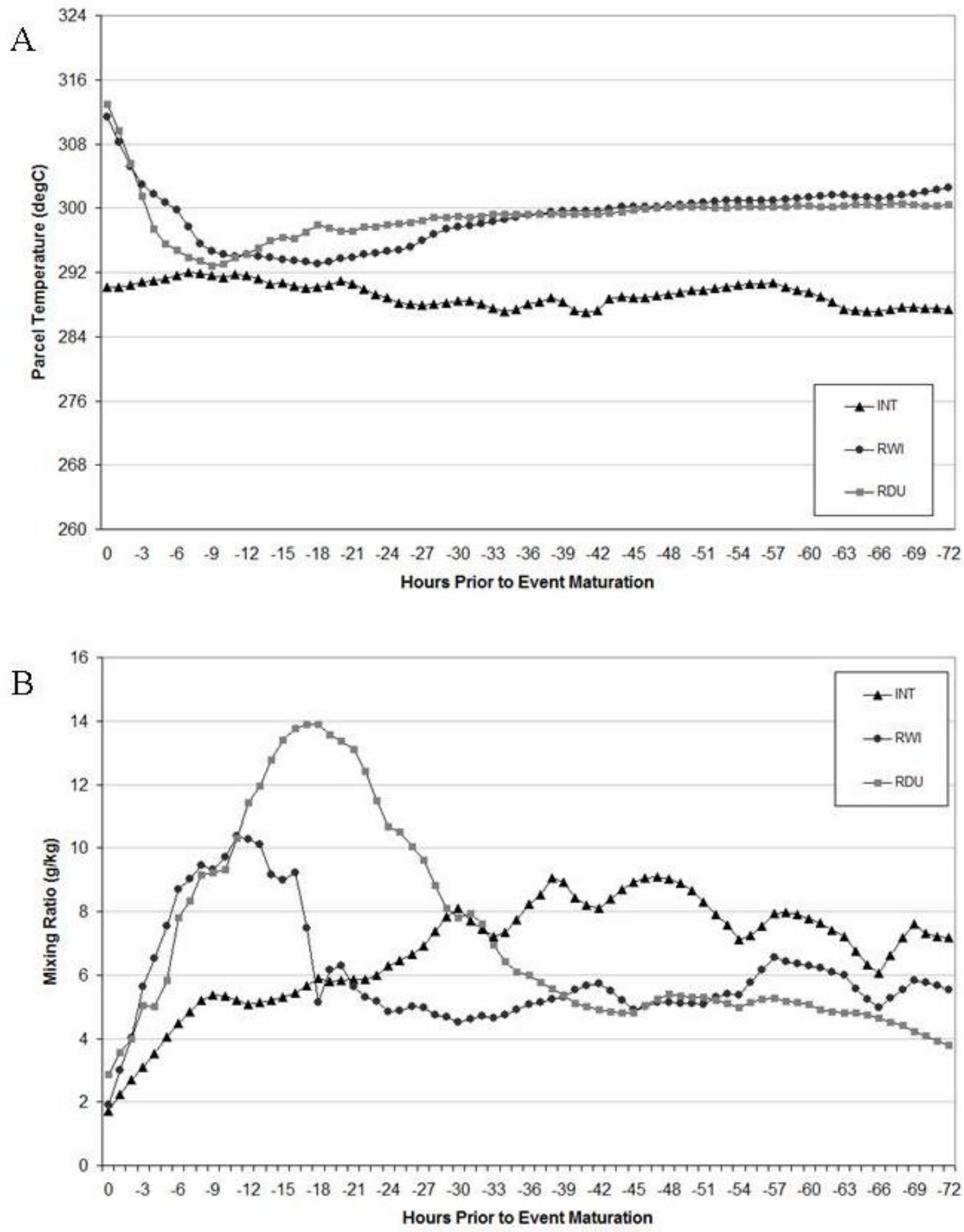

Figure 3.9. Air parcel trajectories of (A) potential temperature and (B) moisture content for 06 UTC 25 January 2000 terminating in the DGZ at RDU, RWI, and INT. 


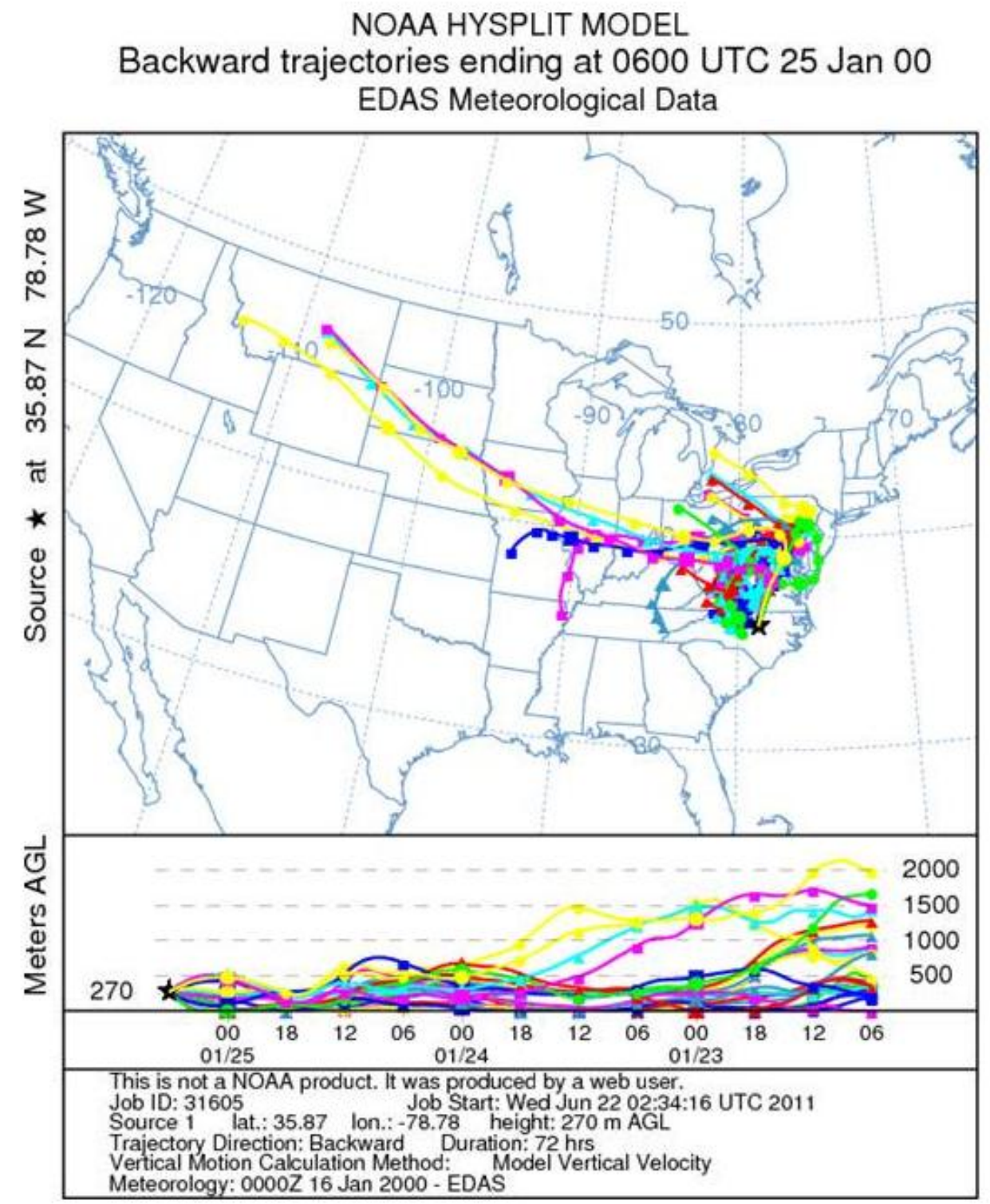

Figure 3.10. Backward ensemble trajectories for 06 UTC 25 January 2000 calculated from RDU at the near-surface level. Vertical trajectories are shown in the bottom panel. 


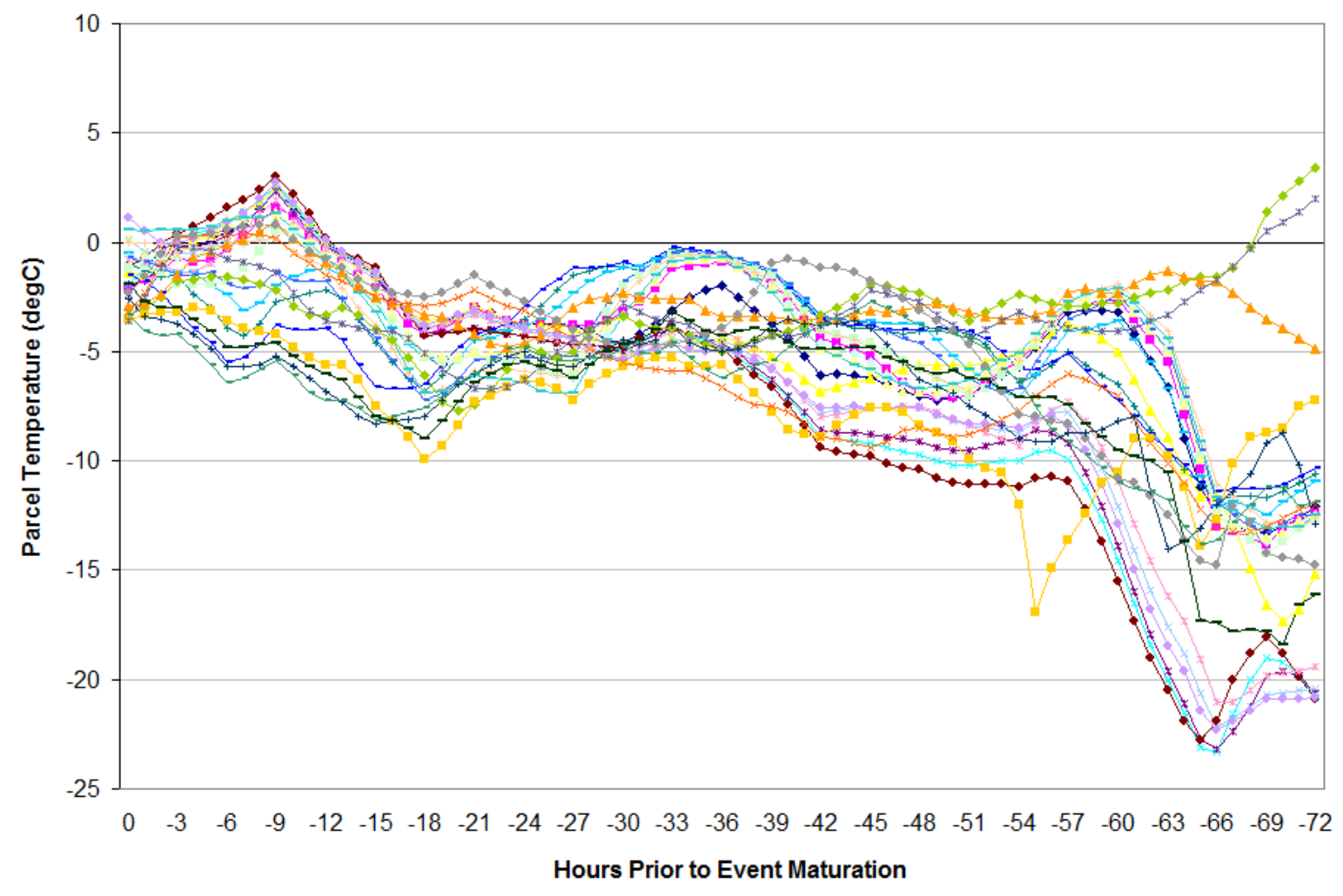

Figure 3.11. Backward ensemble temperature trajectories for 06 UTC 25 January 2000 calculated from RDU at the near-surface level. 


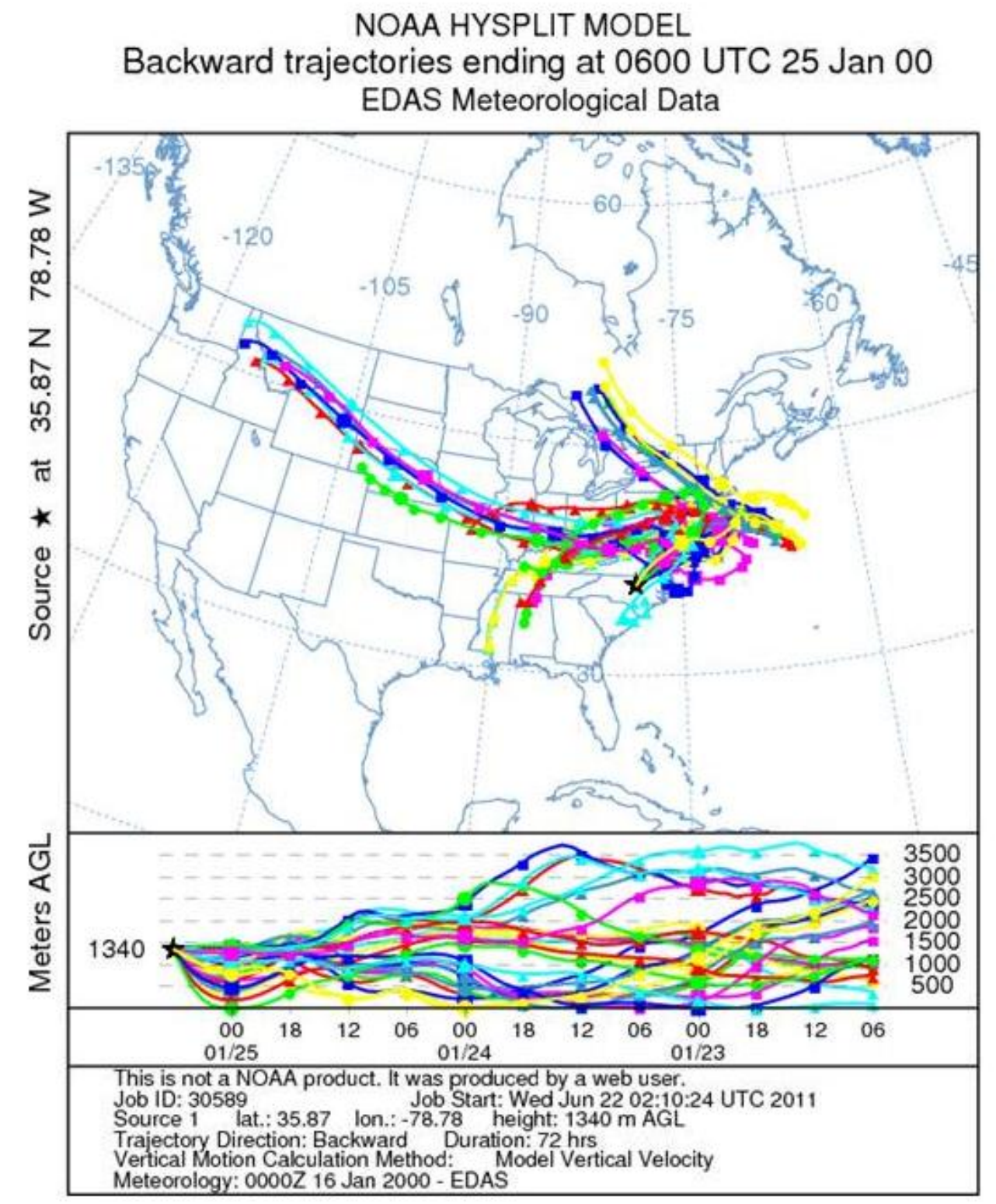

Figure 3.12. Same as Figure 3.10, except at the WLZ. 


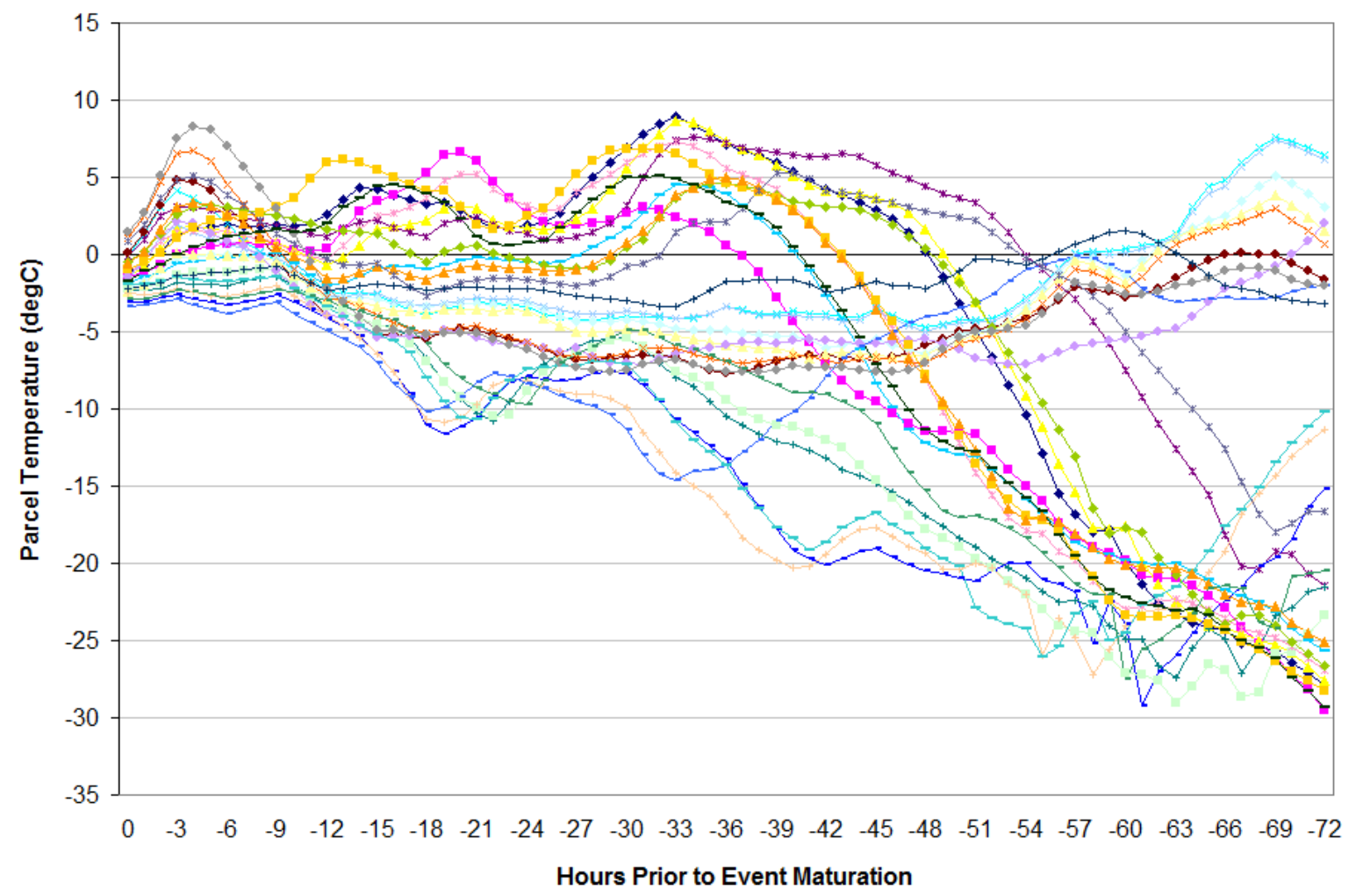

Figure 3.13. Same as Figure 3.11, except at the WLZ. 


\section{(A) Members with $850 \mathrm{hPa} \mathbf{T}>0 \mathbf{d e g C}$}

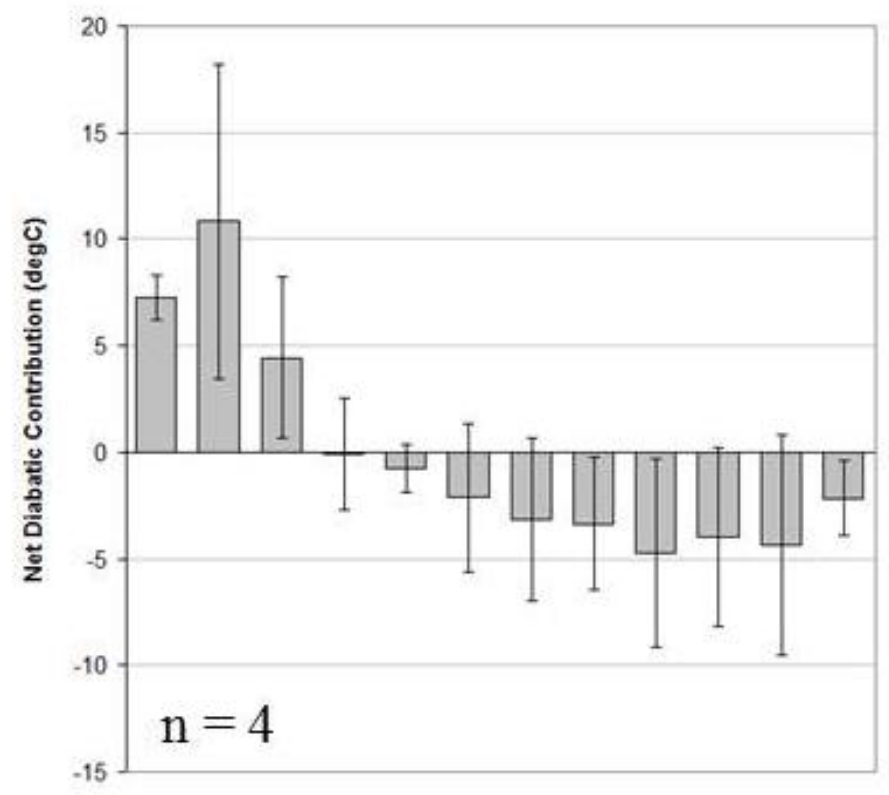

\section{(B) Members with $850 \mathrm{hPa} \mathbf{T}<0 \mathbf{d e g C}$}

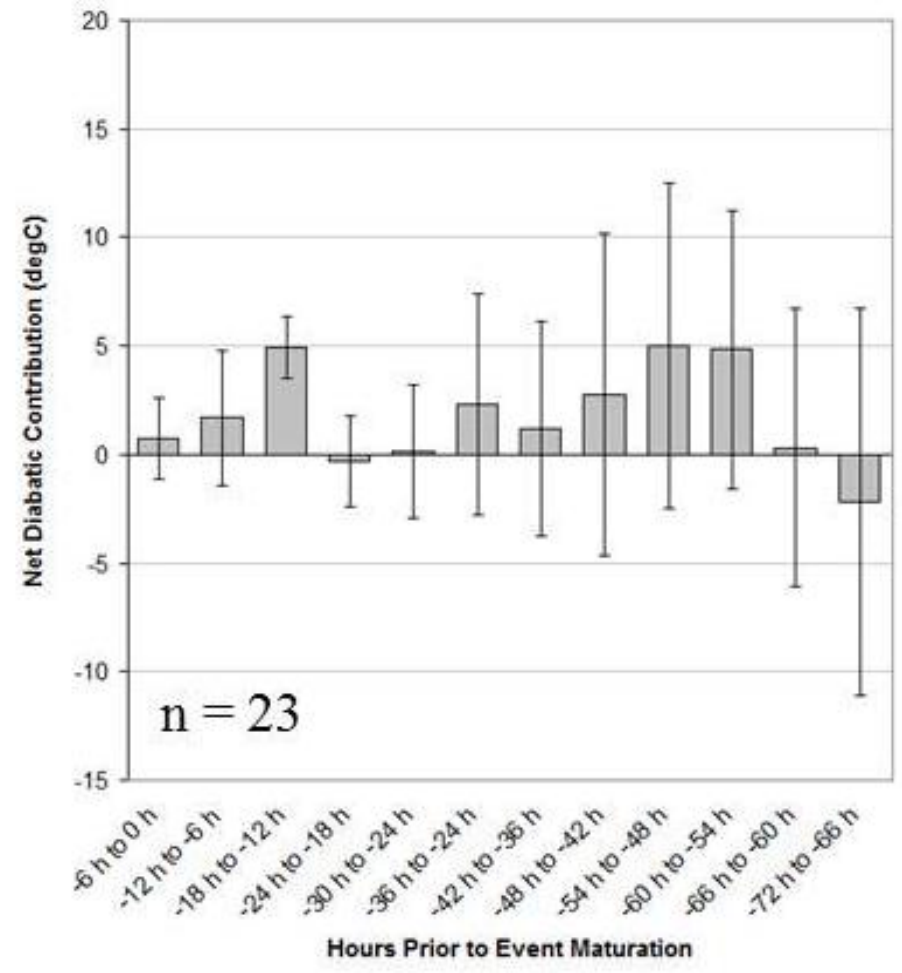

Figure 3.14. Net diabatic contribution to air parcel temperature trajectories at RDU with WLZ temperatures (A) above freezing and (B) below freezing at 6-hr trajectory segments. Range bars indicate the maximum and minimum values among the ensemble members. 


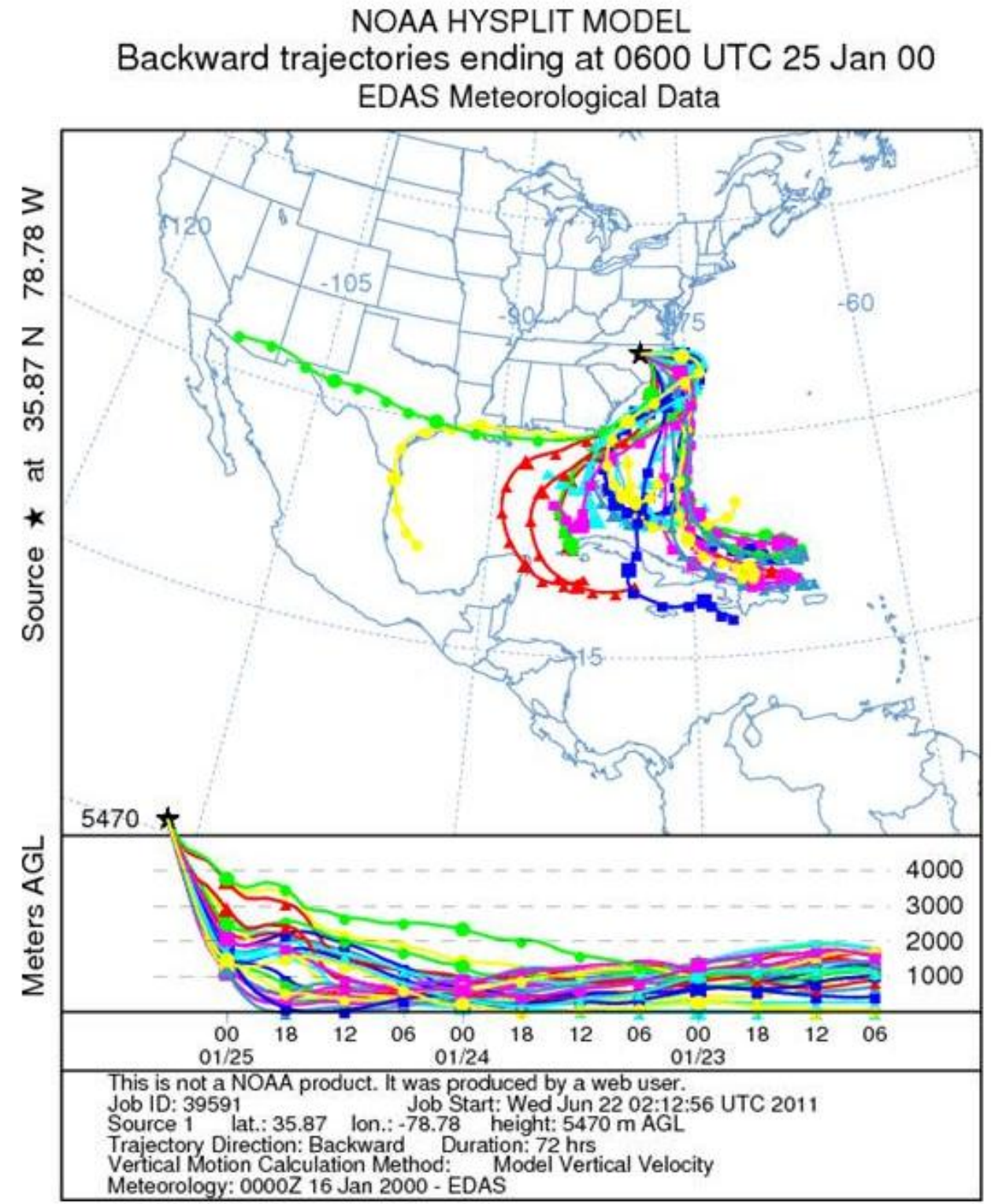

Figure 3.15. Same as Figure 3.10, except at the DGZ. 

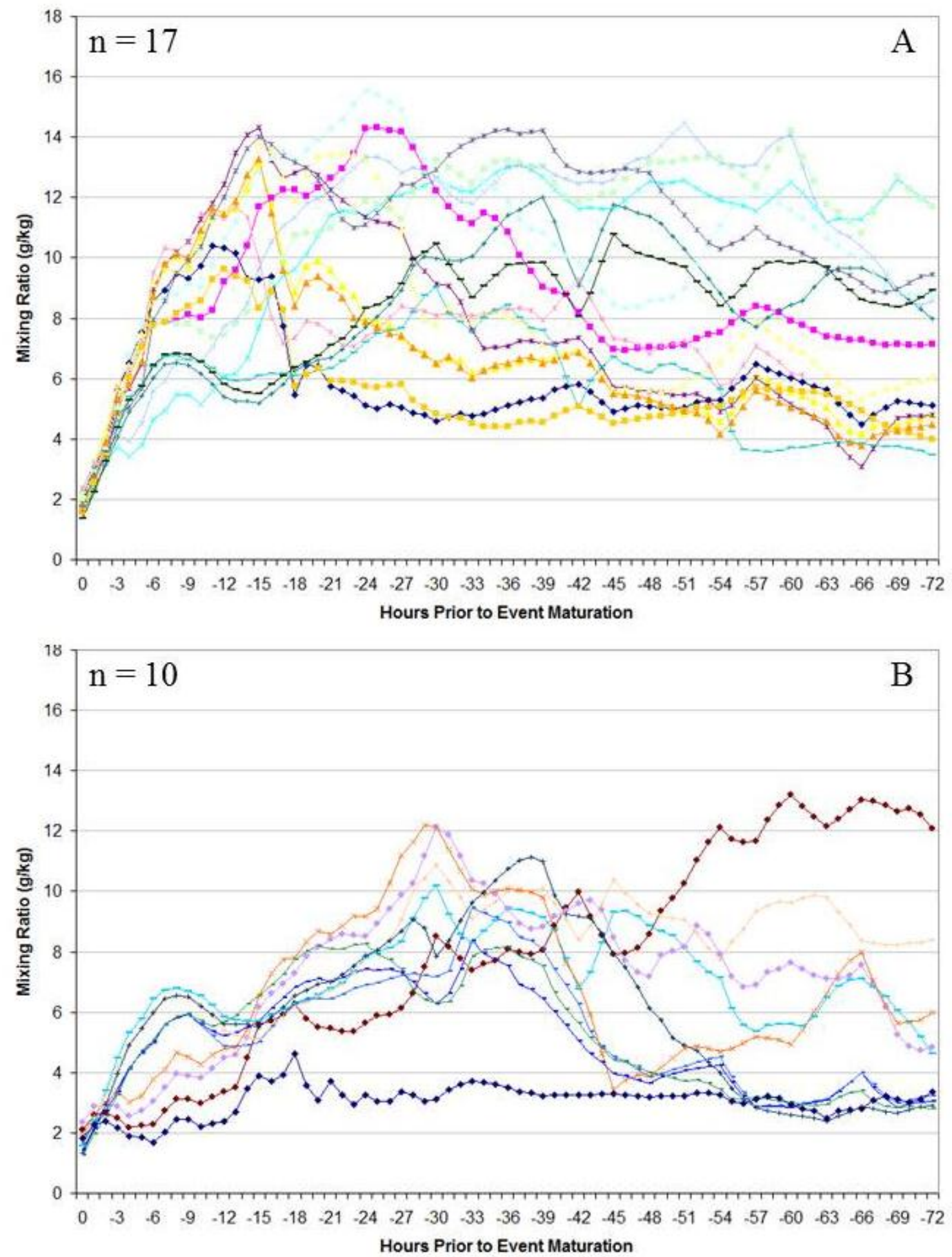

Figure 3.16. Backward ensemble trajectories of moisture content for 06 UTC 25 January 2000 calculated from RDU at the DGZ with origins over the (A) Atlantic Ocean and (B) Gulf of Mexico. 


\subsection{2b. Case II: $1-2$ March 2009}

The snowstorm of 1-2 March 2009 produced 5 to 6 in. (12.7 to $15.2 \mathrm{~cm})$ of snow across the northwestern portion of the Raleigh CWA (see appendix Figure A7). At Greensboro, NC (GSO) the precipitation began as rain on the afternoon of 1 March and then changed over to heavy snow later that evening (Table 3.5). Examination of the observed soundings at GSO revealed that the changeover from liquid to frozen precipitation was associated with a cooling of the WLZ to below freezing (Figure 3.17). This storm was chosen for further analysis to determine the processes that contributed to the cooling of the WLZ and the resulting precipitation changeover. Specifically, a set of back trajectories was calculated during the period of rain (18 UTC 1 March) and during the period of snow following the changeover (06 UTC 2 March).

The WLZ trajectories associated with these two periods of precipitation indicated vastly different source regions, vertical motions, and temperature profiles. During the period of rain, the source of the air was the Gulf of Mexico (Figure 3.18a), whereas the air associated with the period of snow originated near Hudson Bay (Figure 3.18b). Temperature trajectories for the WLZ during the period of rain revealed a gradual cooling of the air over time as it ascended over Mississippi and Alabama. In contrast, temperature trajectories for the period of snow revealed a strong descent and warming of the air over time, while remaining below freezing for the duration of the trajectory. Examination of the net diabatic contribution to the WLZ air parcel temperature revealed a net diabatic cooling of at least $13^{\circ} \mathrm{C}$ during the period of rain over the last 12 hours of the trajectory (Figure 3.19). In contrast, a net diabatic warming of at least $12^{\circ} \mathrm{C}$ was observed during the period of snow over the last 24 hours of the trajectory. The magnitude of these diabatic contributions 
appears to be significant, since the temperature of the air during both periods of precipitation was within just a few degrees of freezing towards the end of each trajectory. In particular, the net diabatic cooling during the period of rain suggests that the cooling of the WLZ and subsequent precipitation-type changeover was driven largely by latent heat absorption due to melting of falling snowflakes.

The ensemble trajectories for this storm were calculated from GSO at 06 UTC 2 March 2009 and correspond with the time and location of heaviest snowfall. The source region of the near-surface ensemble members was the southern tip of Hudson Bay (Figure 3.20). These trajectories exhibited a gradual descent over the first half of the trajectory period, after which they remained generally below $1 \mathrm{~km}$ in altitude. This initial descent was associated with adiabatic warming of air parcels as they approached the region of precipitation (Figure 3.21). Five of the 27 ensemble members exhibited above freezing temperatures at T-0 hours. Figure 3.22 reveals that over the last half of the trajectory, ensemble members with above freezing near-surface temperatures experienced a net diabatic warming, while members with below freezing temperatures exhibited a net diabatic cooling of more than $6^{\circ} \mathrm{C}$ over the last 30 hours of the trajectory. In this case, diabatic cooling due to melting snowflakes may have aided in lowering the freezing level to below $975 \mathrm{hPa}$, creating an environment conducive to snowfall accumulation.

The set of ensemble trajectories terminating in the WLZ is illustrated in Figure 3.23. As with the near-surface ensemble, the trajectories in the WLZ generally converged on a common source region between the Great Lakes and Hudson Bay. The vertical component over the first third of the trajectories was highly variable, which contributed to varying temperatures over this portion of the trajectory period (Figure 3.24). However, all the 
ensemble members terminated at the WLZ with temperatures between -7 and $-10^{\circ} \mathrm{C}$. In this case, the trajectory output for the WLZ was not particularly sensitive to variations in the input data.

Figure 3.25 illustrates the set of ensemble trajectories terminating in the DGZ. Twenty-two of the 27 ensemble members originated across the Great Plains and intermountain West and traveled southward near the Gulf of Mexico before turning cyclonically into the region of precipitation. The mean vertical velocity over the final 6 hours of the trajectory was a modest $-3.7 \mathrm{~cm} / \mathrm{s}$ (range of -2.5 to $-5.8 \mathrm{~cm} / \mathrm{s}$ ). In contrast, the remaining five ensemble members spent considerable time over the Atlantic Ocean and exhibited mean vertical velocities of $-10.4 \mathrm{~cm} / \mathrm{s}$ (range of -9.6 to $-11.4 \mathrm{~cm} / \mathrm{s}$ ). These ensemble members also exhibited much higher moisture contents throughout the trajectory period (Figure 3.26). As in Case I, the decrease in moisture content over the final 12 hours of the trajectory was much greater for the ensemble members over the Atlantic Ocean. These results suggest that the Atlantic Ocean is the largest contributor of moisture to heavy snowstorms in central North Carolina over the 72-hr antecedent trajectory period. 
Table 3.5. Hourly snowfall totals (in liquid equivalent) and present weather observations for the 1-2 March 2009 snowstorm at GSO. Back trajectories were computed from the times highlighted in boldface.

\begin{tabular}{|c|c|c|c|}
\hline Date & $\begin{array}{c}\text { Time } \\
\text { (UTC) }\end{array}$ & $\begin{array}{c}\text { Precipitation } \\
\text { (in Liquid } \\
\text { Equivalent) }\end{array}$ & $\begin{array}{c}\text { Present } \\
\text { Weather* }\end{array}$ \\
\hline $\mathbf{0 3 / 0 1 / 2 0 0 9}$ & $\mathbf{1 8}$ & $\mathbf{0 . 1 1}$ & RA BR \\
\hline $03 / 01 / 2009$ & 19 & 0.13 & RA BR \\
\hline $03 / 01 / 2009$ & 20 & 0.16 & RA BR \\
\hline $03 / 01 / 2009$ & 21 & 0.07 & -RA \\
\hline $03 / 01 / 2009$ & 22 & 0.01 & -RA \\
\hline $03 / 01 / 2009$ & 23 & Tr & -DZ \\
\hline $03 / 02 / 2009$ & 00 & 0.01 & -RA BR \\
\hline $03 / 02 / 2009$ & 01 & 0.03 & -SN \\
\hline $03 / 02 / 2009$ & 02 & 0.03 & -SN \\
\hline $03 / 02 / 2009$ & 03 & 0.04 & -SN \\
\hline $03 / 02 / 2009$ & 04 & 0.03 & -SN \\
\hline $03 / 02 / 2009$ & 05 & 0.05 & -SN \\
\hline $\mathbf{0 3 / 0 2 / 2 0 0 9}$ & $\mathbf{0 6}$ & $\mathbf{0 . 1 0}$ & -SN \\
\hline $03 / 02 / 2009$ & 07 & 0.05 & -SN \\
\hline $03 / 02 / 2009$ & 08 & 0.01 & -SN \\
\hline $03 / 02 / 2009$ & 09 & Tr & -SN BR \\
\hline $03 / 02 / 2009$ & 10 & Tr & -SN BR \\
\hline $03 / 02 / 2009$ & 11 & Tr & -SN BR \\
\hline
\end{tabular}

$* \mathrm{RA}=$ rain $; \mathrm{BR}=$ mist $; \mathrm{DZ}=$ drizzle $; \mathrm{SN}=$ snow 

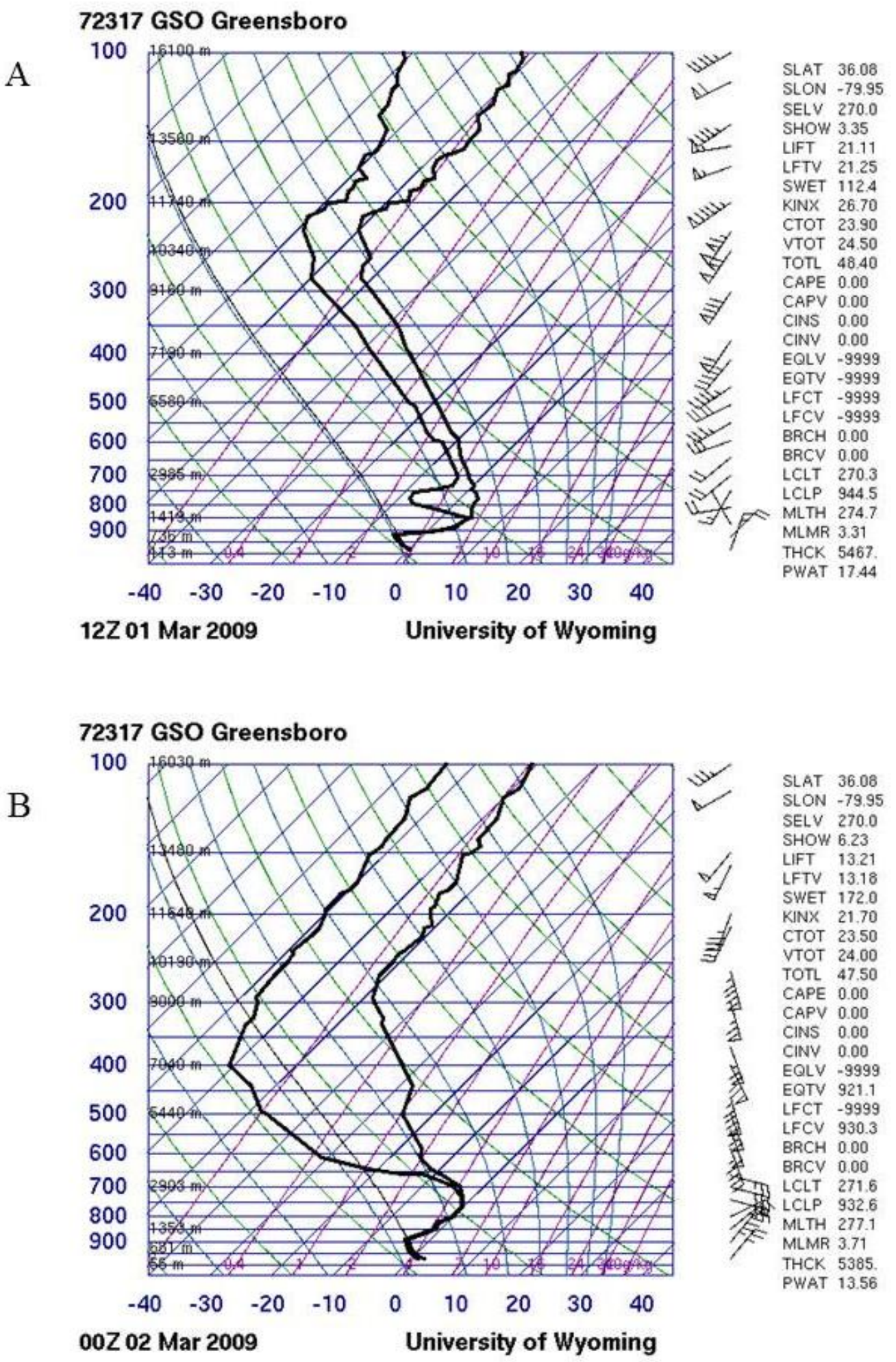

Figure 3.17. Observed soundings from Greensboro, NC (GSO) at (A) 12 UTC 1 March 2009 and (B) 00 UTC 2 March 2009. 

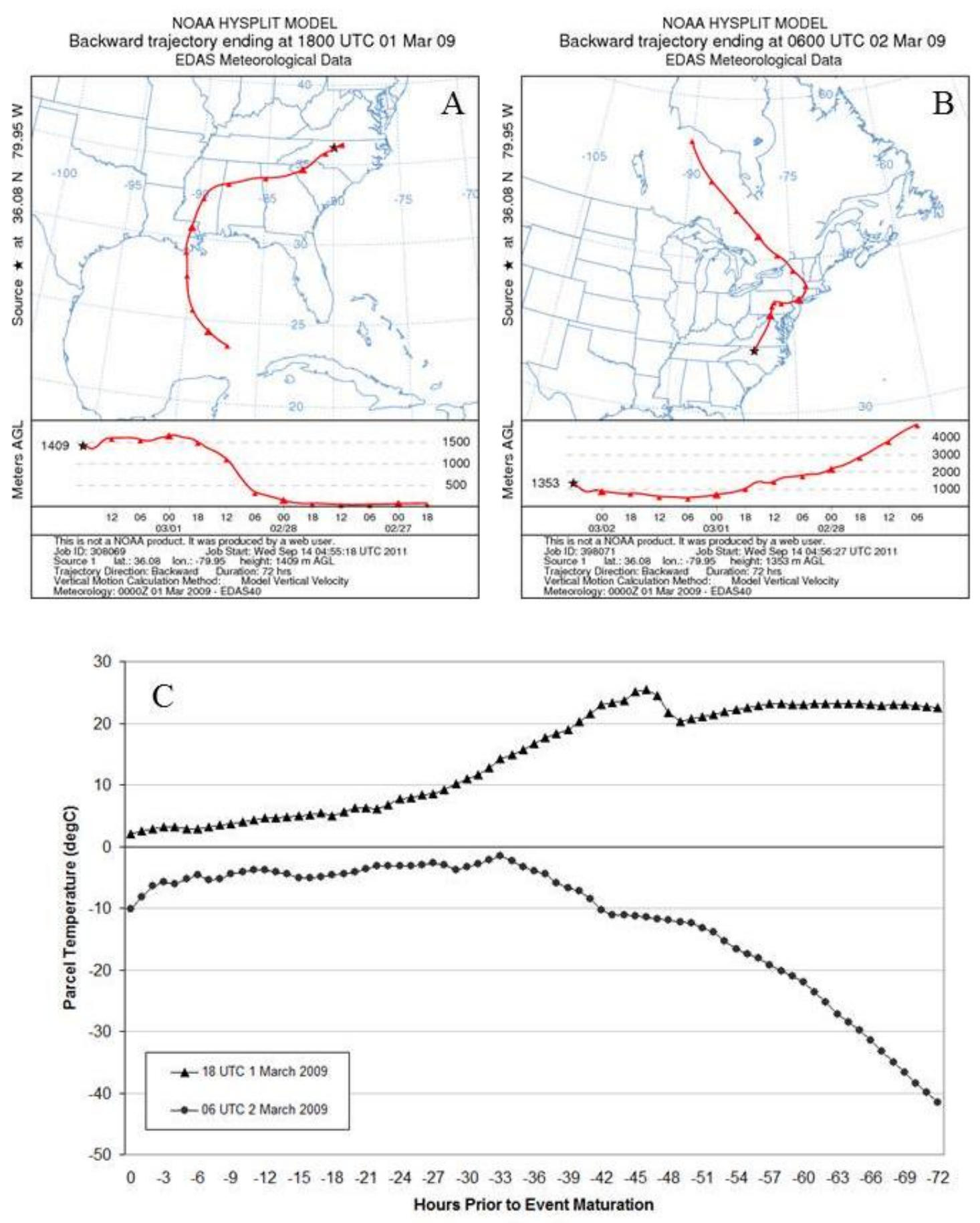

Figure 3.18. Horizontal and vertical trajectories for (A) 18 UTC 1 March 2009 and (B) 06 UTC 2 March 2009 terminating at the WLZ at GSO. (C) Corresponding air parcel temperature trajectories. 


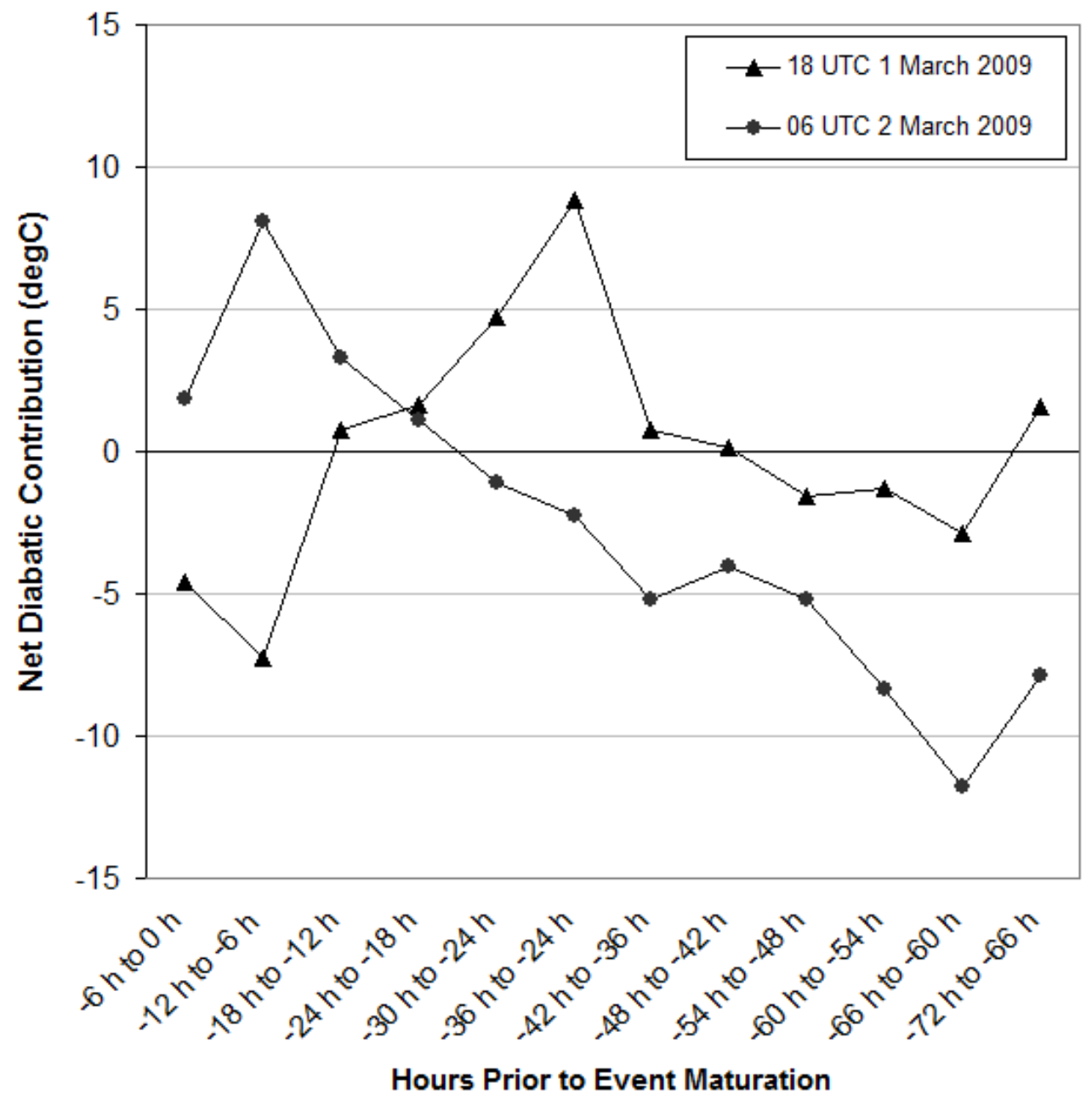

Figure 3.19. Net diabatic contribution to air parcel temperature trajectories at GSO for 18 UTC 1 March 2009 (rain) and 06 UTC 2 March 2009 (snow) at 6-hr trajectory segments. 


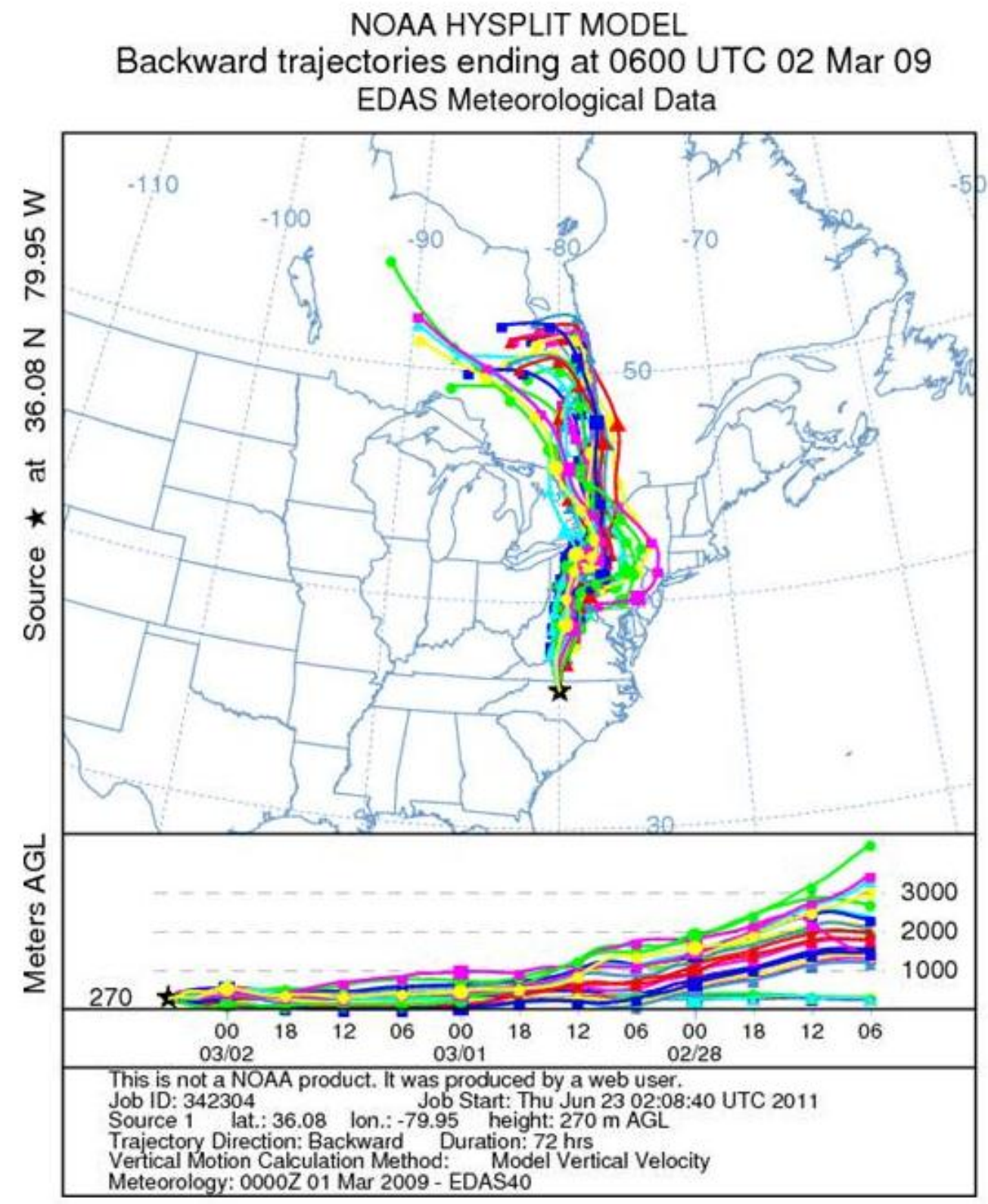

Figure 3.20. Backward ensemble trajectories for 06 UTC 2 March 2009 calculated from GSO at the near-surface level. Vertical trajectories are shown in the bottom panel. 


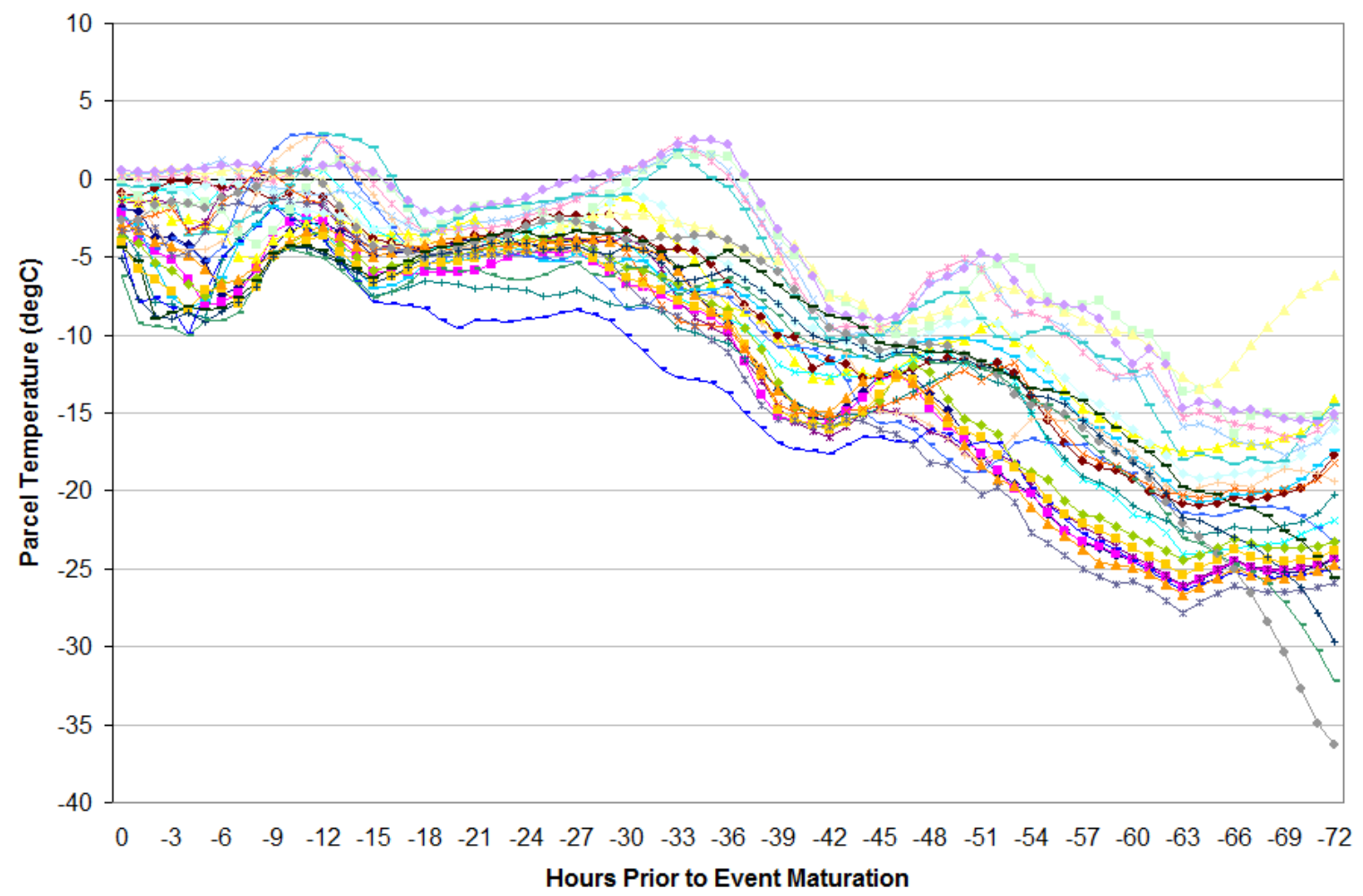

Figure 3.21. Backward ensemble temperature trajectories for 06 UTC 2 March 2009 calculated from GSO at the near-surface level. 


\section{(A) Members with $975 \mathrm{hPa} \mathbf{T}>\mathbf{0} \operatorname{degC}$}

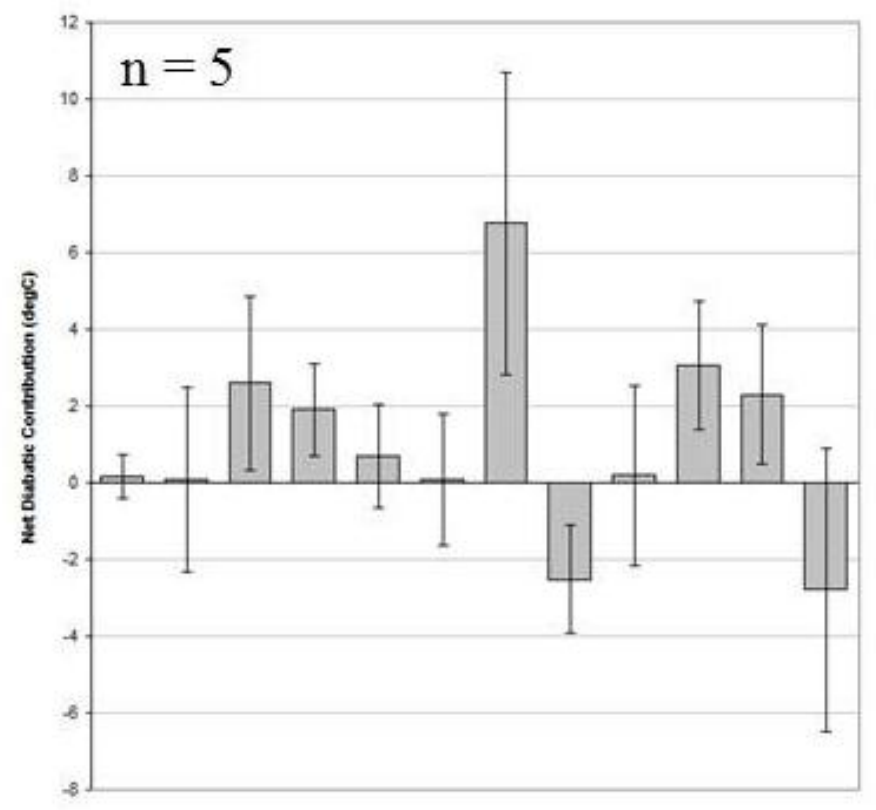

\section{(B) Members with $975 \mathrm{hPa} \mathbf{T}<\mathbf{0} \mathbf{d e g C}$}

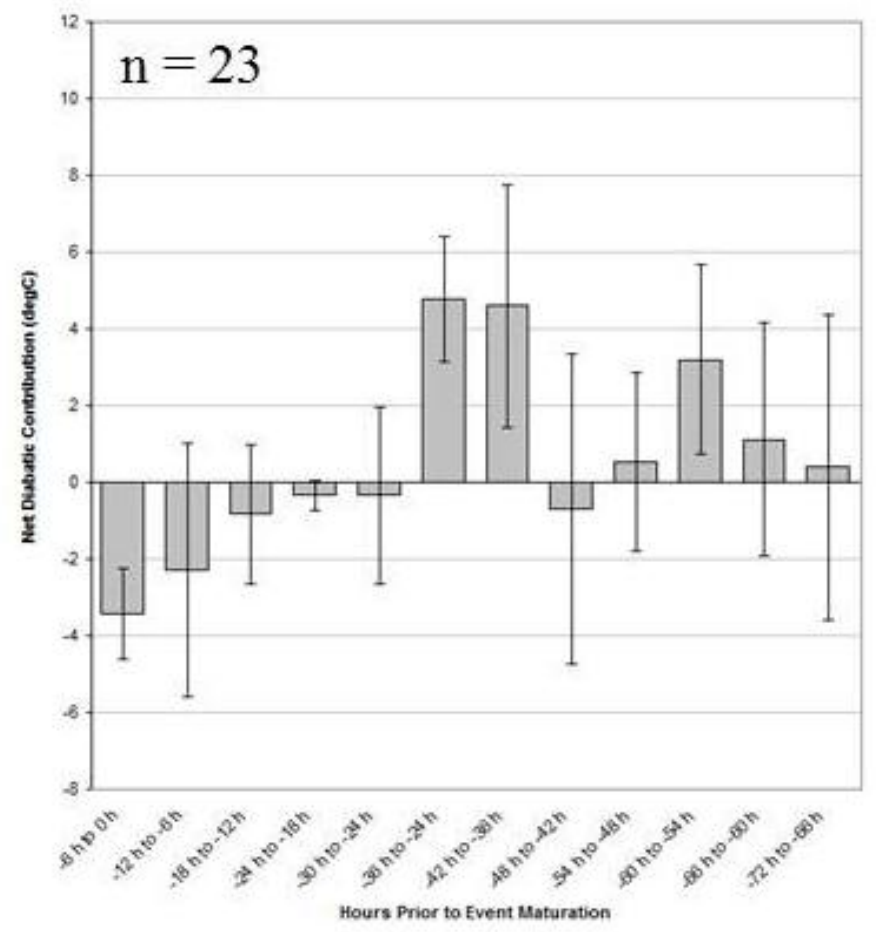

Figure 3.22. Net diabatic contribution to air parcel temperature trajectories at GSO with nearsurface temperatures (A) above freezing and (B) below freezing at 6-hr trajectory segments. Range bars indicate the maximum and minimum values among the ensemble members. 


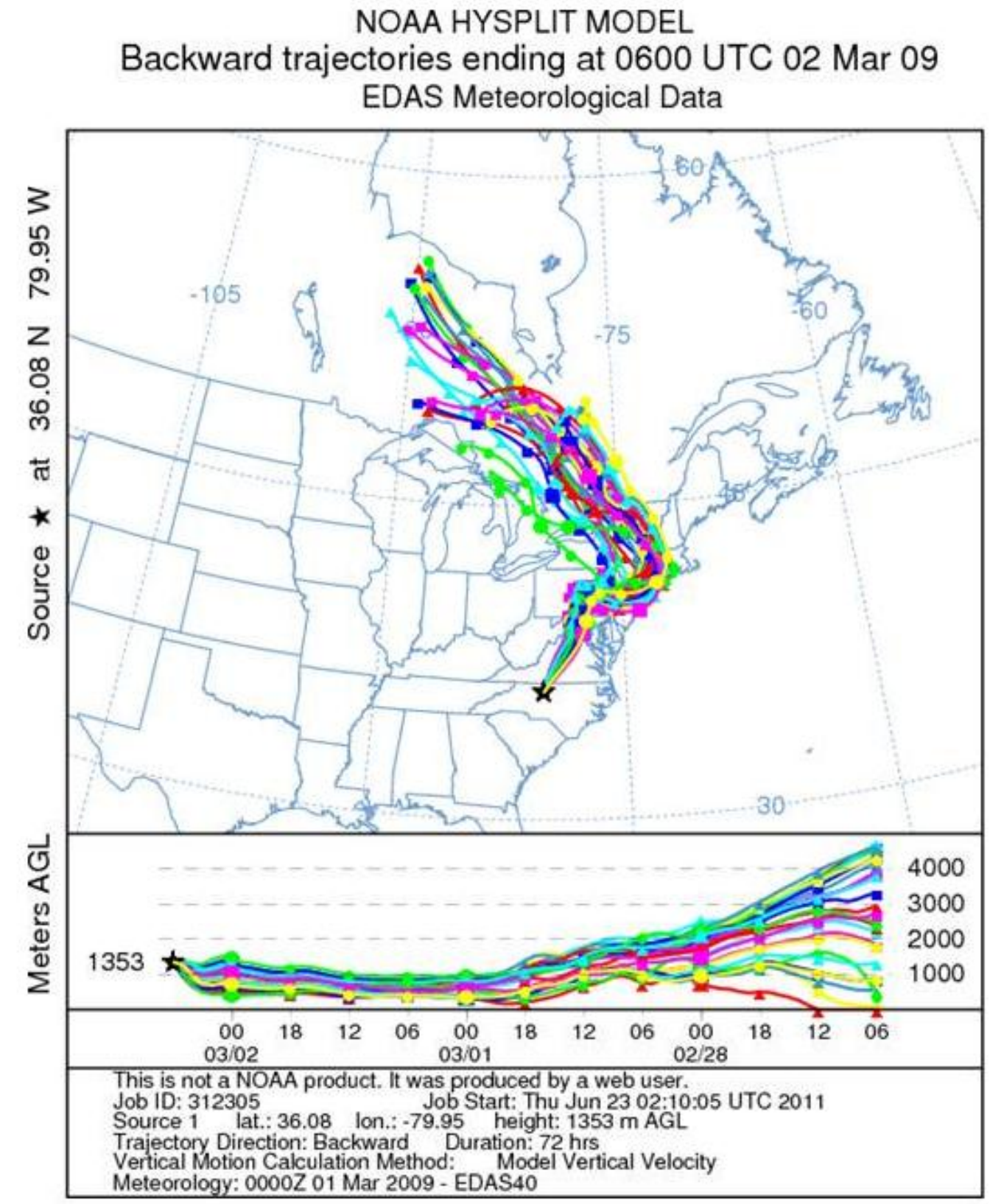

Figure 3.23. Same as Figure 3.20, except at the WLZ. 


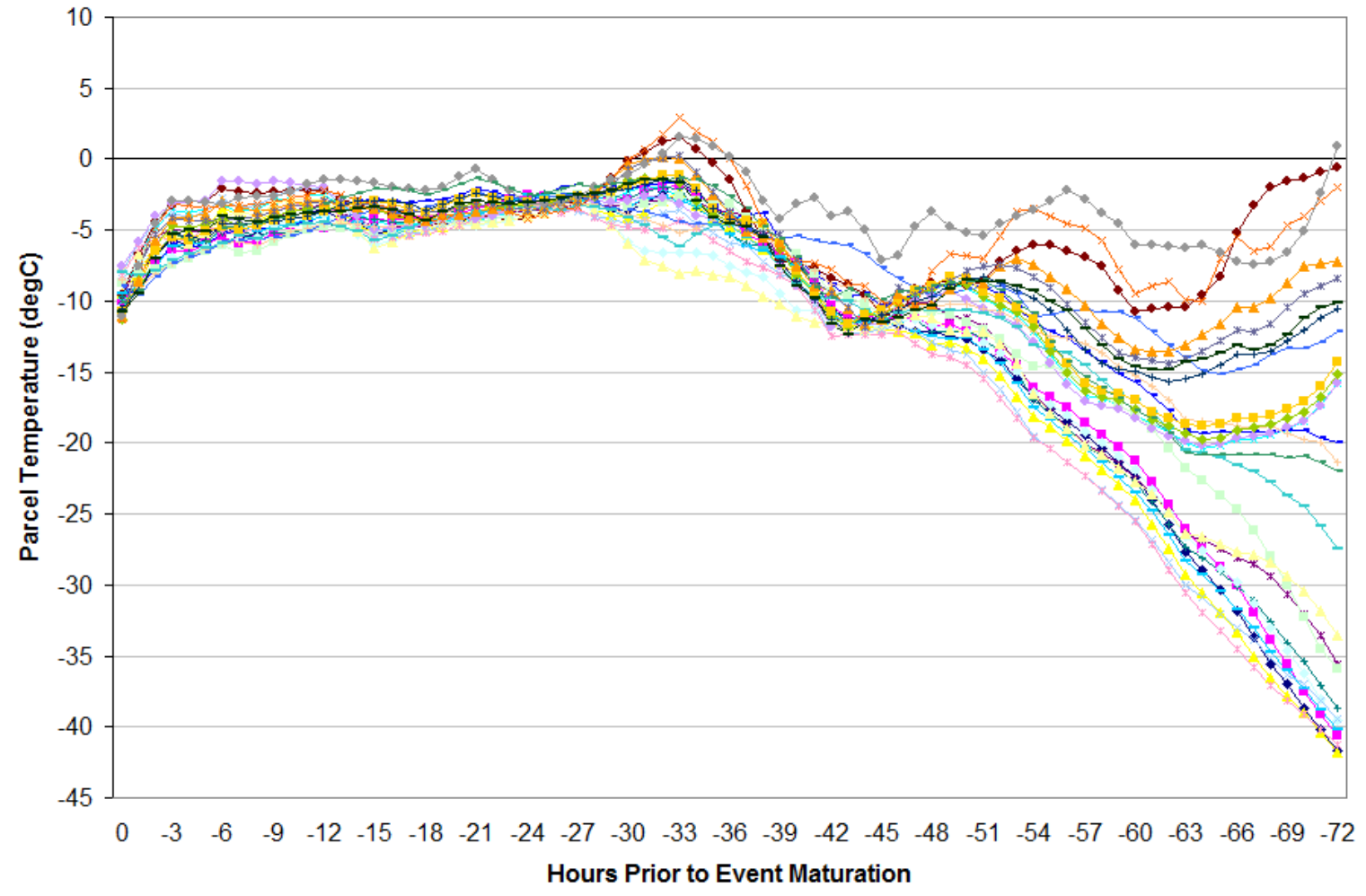

Figure 3.24. Same as Figure 3.21, except at the WLZ. 


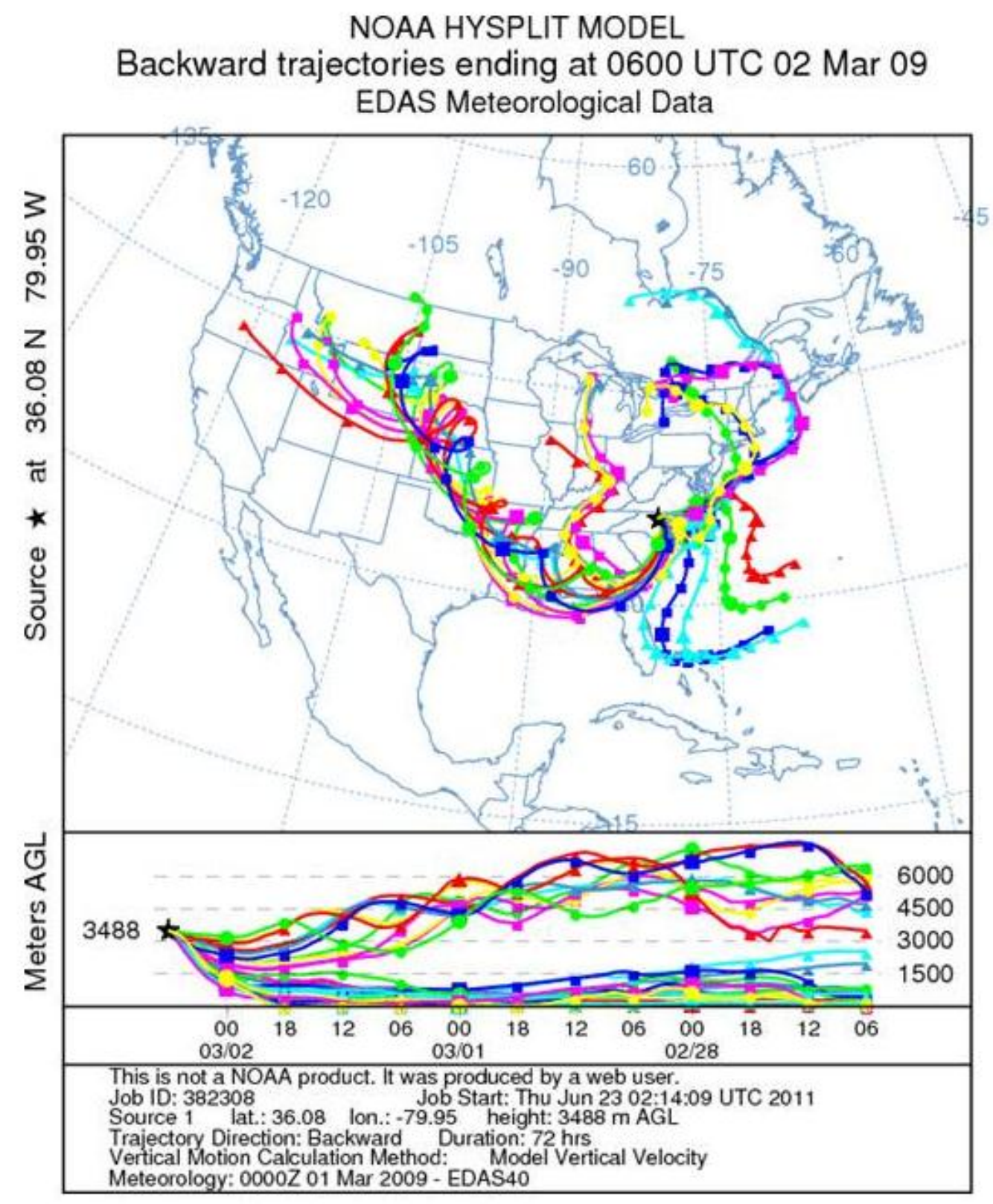

Figure 3.25. Same as Figure 3.20, except at the DGZ. 

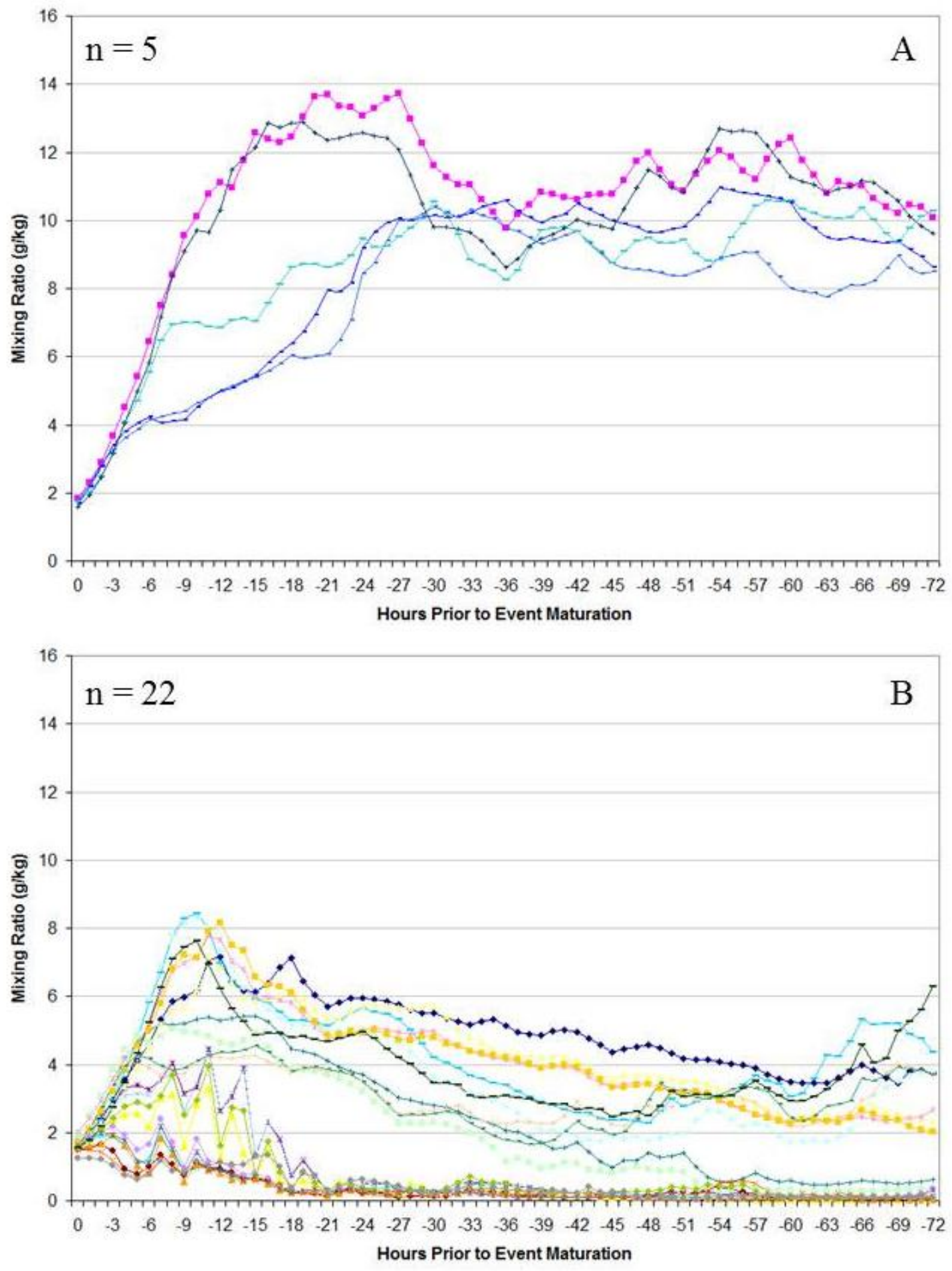

Figure 3.26. Backward ensemble moisture trajectories for 06 UTC 2 March 2009 calculated from GSO at the DGZ with origins over the (A) Atlantic Ocean and (B) central United States. 


\subsection{Synoptic Analyses}

This section examines the relationship between the antecedent synoptic-scale circulation and the space-time character of the composite back trajectories at 24-hour intervals. Analysis of the vertical component of the back trajectories revealed that each trajectory type remained below $2 \mathrm{~km}$ over the course of the 72-hr trajectory period (see Figure 3.2). Therefore, composites of low-level geopotential height fields (i.e. 1000 and 850 $\mathrm{hPa}$ ) were used to assess the aspects of the synoptic-scale circulation responsible for the back trajectory motions. The composite $1000 \mathrm{hPa}$ geopotential height field provides an approximation of the surface pressure pattern. Comparisons are also made between snowstorms characterized by DGZ-cyclonic and DGZ-anticyclonic trajectories.

\subsubsection{Near-Surface Trajectory}

As was previously illustrated, the near-surface trajectory exhibited anticyclonic curvature and gradual descent over the 72 -hr trajectory period. This trajectory was located along the eastern periphery of a surface anticyclone ridging southward out of Canada in the wake of a strong cyclone departing the Northeast U.S. (Figure 3.27). Between T-48 and T24 hours, the near-surface trajectory turned anticyclonically in response to $1000 \mathrm{hPa}$ geopotential height rises over the Great Lakes and the development of a weak area of low pressure over the eastern Gulf of Mexico. At T-24 hours, a well-defined cyclone was located off the Southeast coast with an anticyclone centered over the Great Plains. A tight gradient in the $1000 \mathrm{hPa}$ geopotential height field extended from northeast to southwest between these surface features. As a result, the near-surface trajectory turned to the southwest over the final 24 hours of the trajectory period. Because the near-surface trajectory was located along the 
periphery rather than within the core of the anticyclone, its vertical descent was relatively modest (see Figure 3.2). This limited the amount of adiabatic warming along the trajectory, therefore maintaining an air parcel temperature supportive of snowfall (i.e. $<0^{\circ} \mathrm{C}$ ).

\subsubsection{Warm Layer Zone Trajectory}

The composite $850 \mathrm{hPa}$ circulation from T-72 to T-48 hours exhibited northwesterly flow across the Great Lakes region in response to a departing surface cyclone, with a weak composite trough extending down the western portion of the Appalachians (Figure 3.28). This circulation pattern corresponded well with the southeastward-curving WLZ trajectory between T-72 and T-48 hours. However, between T-48 and T-24 hours, the WLZ trajectory abruptly turned southwestward and crossed the Delmarva Peninsula before entering central North Carolina. Interestingly, this shift in trajectory motion occurred as the $850 \mathrm{hPa}$ trough deepened across Tennessee and Alabama (Figure 3.28) and the intensifying surface cyclone moved northeastward out of the eastern Gulf of Mexico (Figure 3.27). This suggests that the WLZ trajectory followed the $850 \mathrm{hPa}$ flow early in the trajectory period, away from the influence of strong dynamics (e.g. troughs, closed lows, surface cyclones). However, as the WLZ trajectory descended offshore to an altitude below $850 \mathrm{hPa}$, it was entrained into the strengthening circulation connected with the intensifying surface cyclone. At the time of the trajectory shift, the horizontal distance between the WLZ air parcel and the surface cyclone was approximately $1500 \mathrm{~km}$. Between T-24 and T-0 hours, the $850 \mathrm{hPa}$ trough amplified into a closed low in the vicinity of the surface cyclone, while the WLZ trajectory continued to travel cyclonically in response to the deepening cyclone immediately downstream. 


\subsubsection{Dendritic Growth Zone Trajectories}

Variations in the 1000 and $850 \mathrm{hPa}$ geopotential height fields between snowstorms with DGZ-anticyclonic and DGZ-cyclonic trajectories were examined at T-24 and T-0 hours. These times were chosen because the most distinguishing aspects of the synoptic-scale circulation occurred over the final 24 hours of the trajectory period. As previously shown, the DGZ-anticyclonic trajectory originated near the Great Lakes and extended off the midAtlantic coast before re-curving into central North Carolina. In contrast, the DGZ-cyclonic trajectory originated over the Caribbean Sea and remained off the Southeast coast as it traveled northward. The most distinguishing characteristic of the low-level synoptic-scale circulation between the two DGZ trajectory types was the development and intensification of the surface cyclone. Snowstorms with a DGZ-anticyclonic trajectory underwent initial cyclogenesis over the eastern Gulf of Mexico at T-24 hours, followed by a gradual deepening and intensification of the cyclone off the Southeast coast (Figure 3.29). These processes coincided with a deepening $850 \mathrm{hPa}$ trough and eventual amplification into a closed low at T0 hours.

In contrast, snowstorms with a DGZ-cyclonic trajectory underwent rapid cyclogenesis between T-24 and T-0 hours (Figure 3.30). The composite $1000 \mathrm{hPa}$ geopotential height field revealed a closed circulation off the Southeast coast at T-0 hours with heights at least 50 m lower compared to the DGZ-anticyclonic composite. The stronger surface cyclone was associated with a deeper $850 \mathrm{hPa}$ trough and closed low at T-24 and T-0 hours, respectively (Figure 3.30). The rapid deepening and intensification of the surface cyclone associated with the DGZ-cyclonic trajectory was tied to the amplification of a $500 \mathrm{hPa}$ trough across the southern U.S. (not shown). Embedded within the trough was a jet streak with winds in 
excess of $35 \mathrm{~m} / \mathrm{s}$. A diffluent exit region associated with the jet streak was located over northern Florida where the surface cyclone developed between T-24 and T-0 hours.

These results suggest that strong cyclones characterized by rapid development and intensification along the Southeast coast are able to draw warm, moist air from tropical latitudes (i.e. south of $25^{\circ} \mathrm{N}$ ) into the DGZ across central North Carolina during at least some heavy snowstorms (i.e. DGZ-cyclonic trajectory). In contrast, snowstorms with weaker and early-developing cyclones are likely to draw air from more northern latitudes with as much as 50 percent less moisture (i.e. DGZ-anticyclonic trajectory; see Figure 3.4). As a result, snowstorms with DGZ-cyclonic trajectories exhibited higher maximum precipitation rates (0.05 to $0.12 \mathrm{in} / \mathrm{hr}$ ) than snowstorms with DGZ-anticyclonic trajectories (0.04 to $0.07 \mathrm{in} / \mathrm{hr})$. 

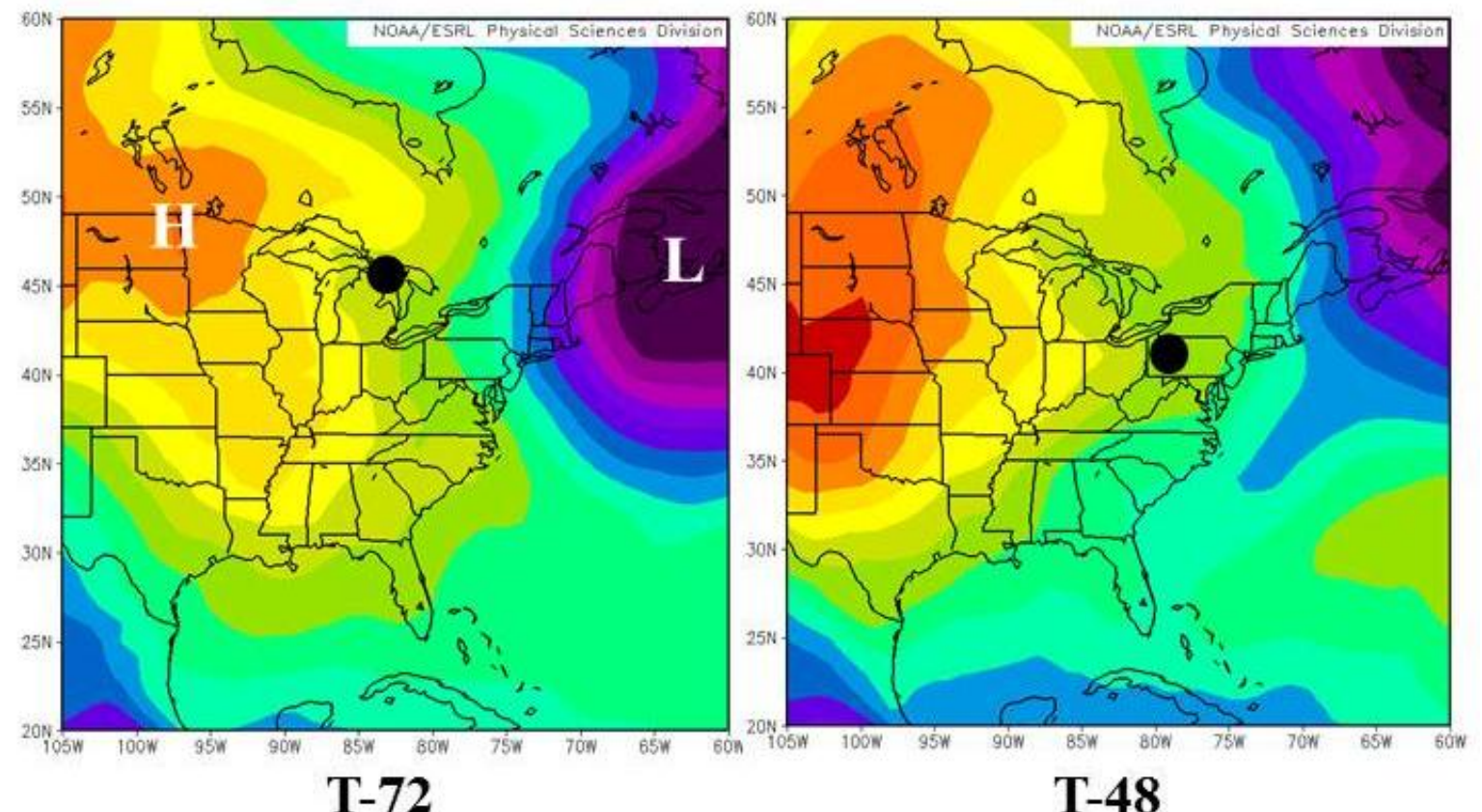

T-72

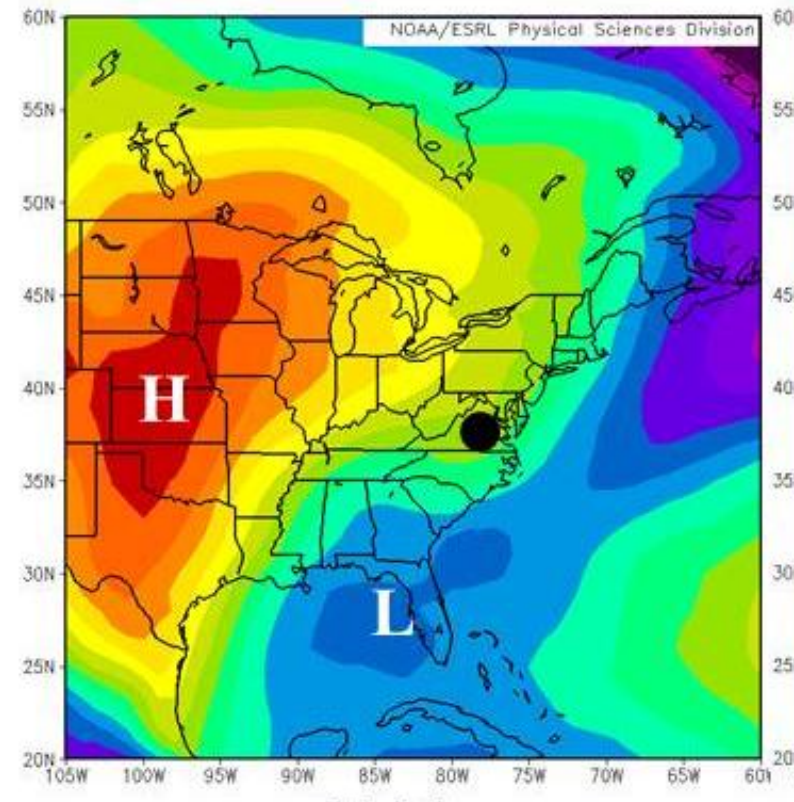

T-24

\section{T-48}

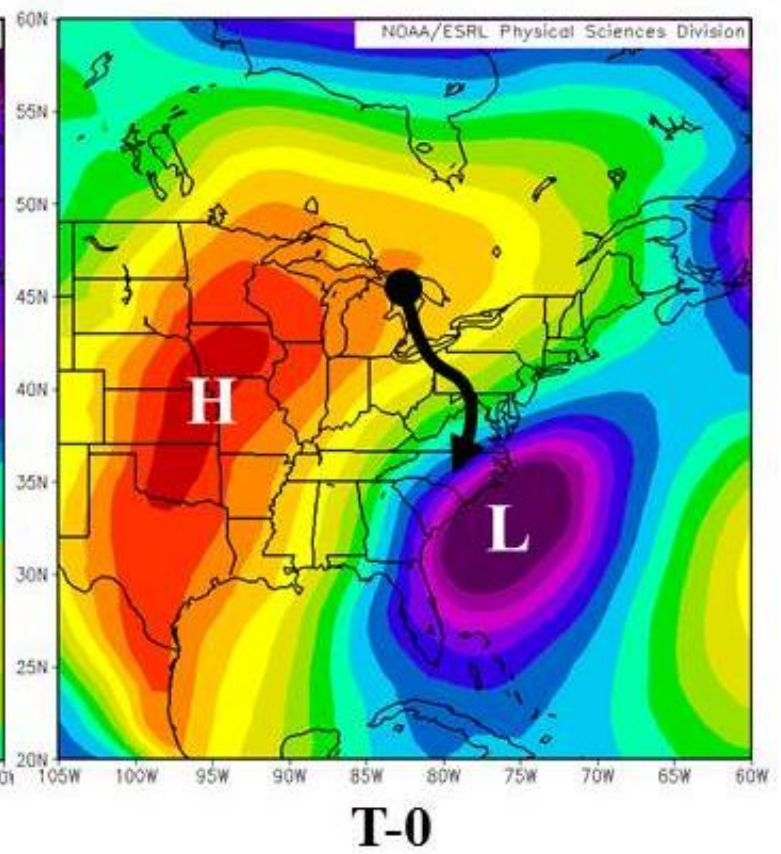

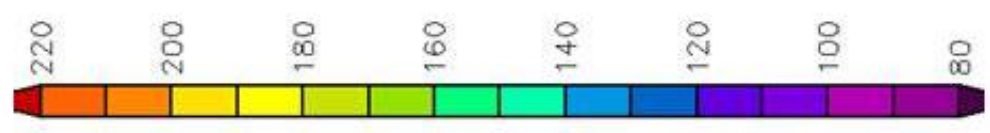

Figure 3.27. Composite analysis of $1000 \mathrm{hPa}$ geopotential heights (meters) for all eight snowstorms at 24-hr intervals from T-72 to T-0 hours. Areas of high pressure ("H") and low pressure ("L") are noted. The position of the air parcel at each time step is denoted by a black circle. A sketch of the 72-hr composite near-surface trajectory is denoted with a solid black line and arrow at T-0 hours. 


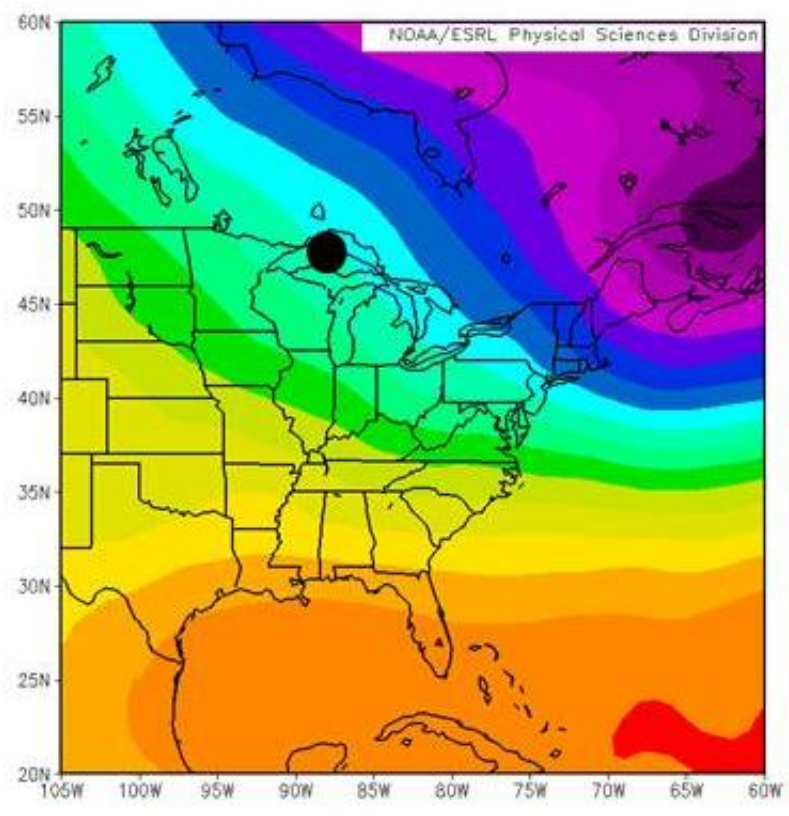

T-72

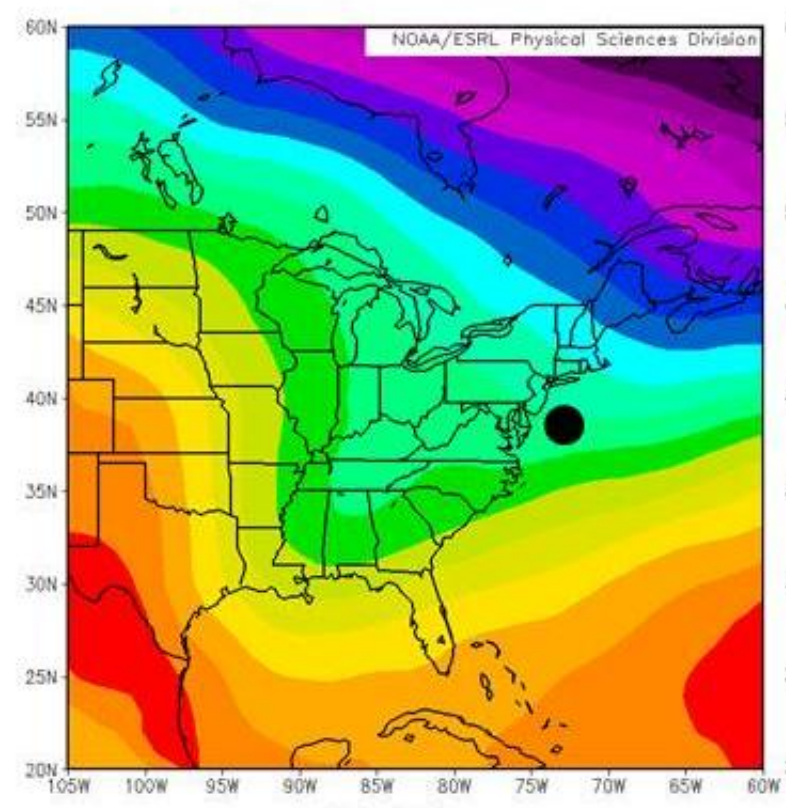

T-24

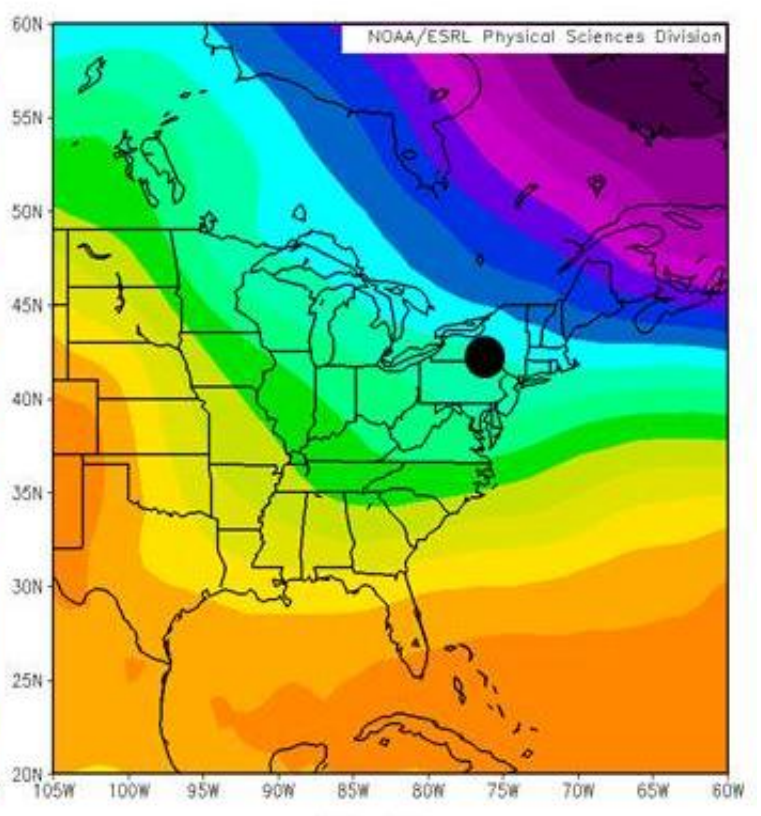

T-48

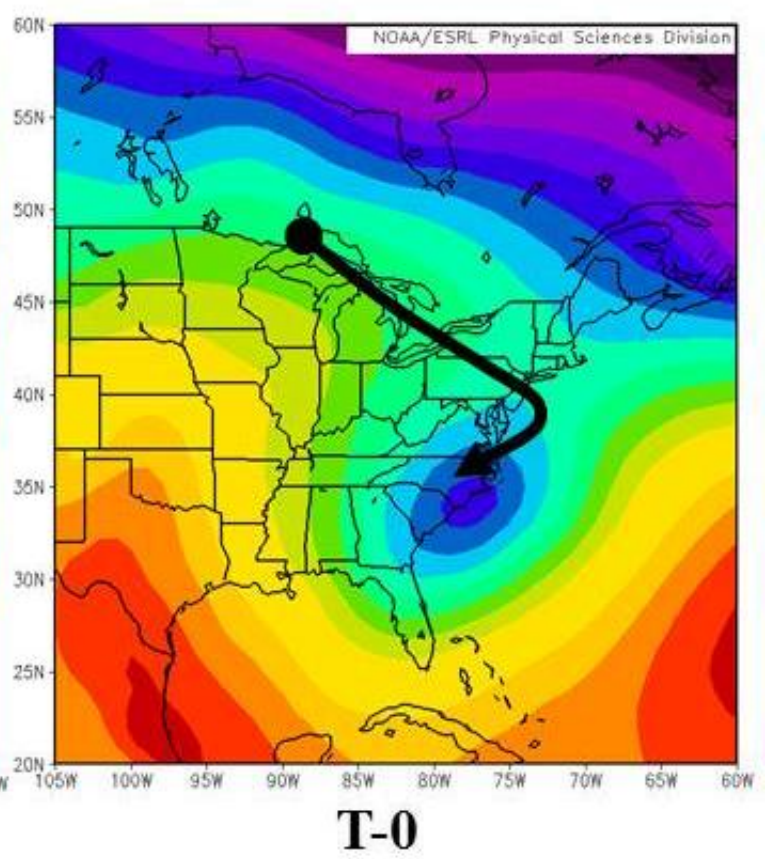

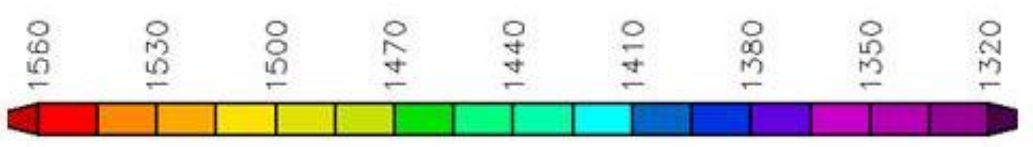

Figure 3.28. Same as Figure 3.27, except of $850 \mathrm{hPa}$ geopotential heights (meters). The position of the air parcel at each time step is denoted by a black circle. A sketch of the composite WLZ trajectory is denoted with a solid black line and arrow. 

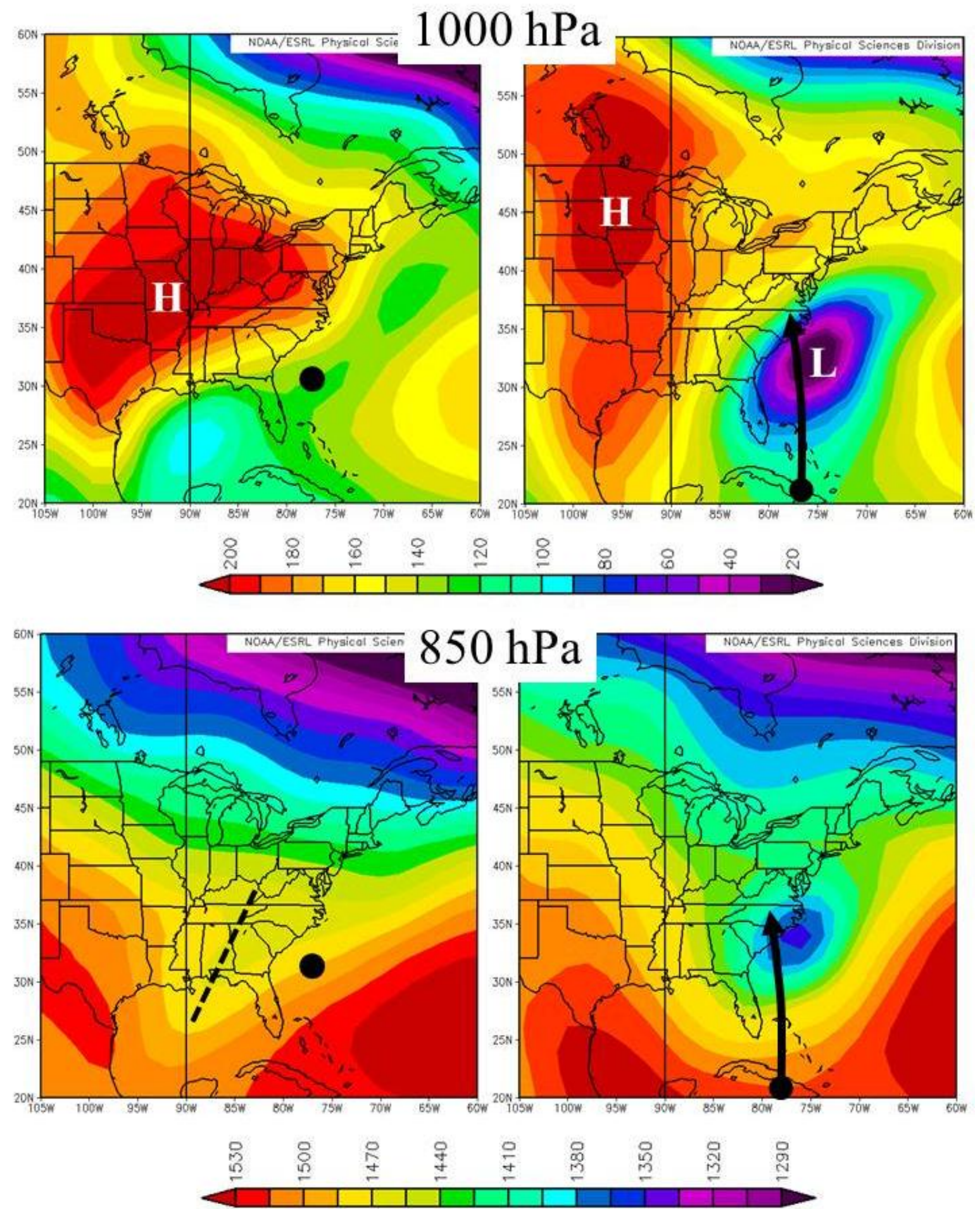

Figure 3.29. Composite analysis of 1000 and $850 \mathrm{hPa}$ geopotential heights (meters) for snowstorms with DGZ-anticyclonic trajectories at T-24 hours (left column) and T-0 hours (right column). The $850 \mathrm{hPa}$ trough axis at T-24 hours is denoted by the black dashed line. The position of the air parcel at each time step is denoted by a black circle. A sketch of the composite DGZ-anticyclonic trajectory is denoted with a solid black line and arrow. 

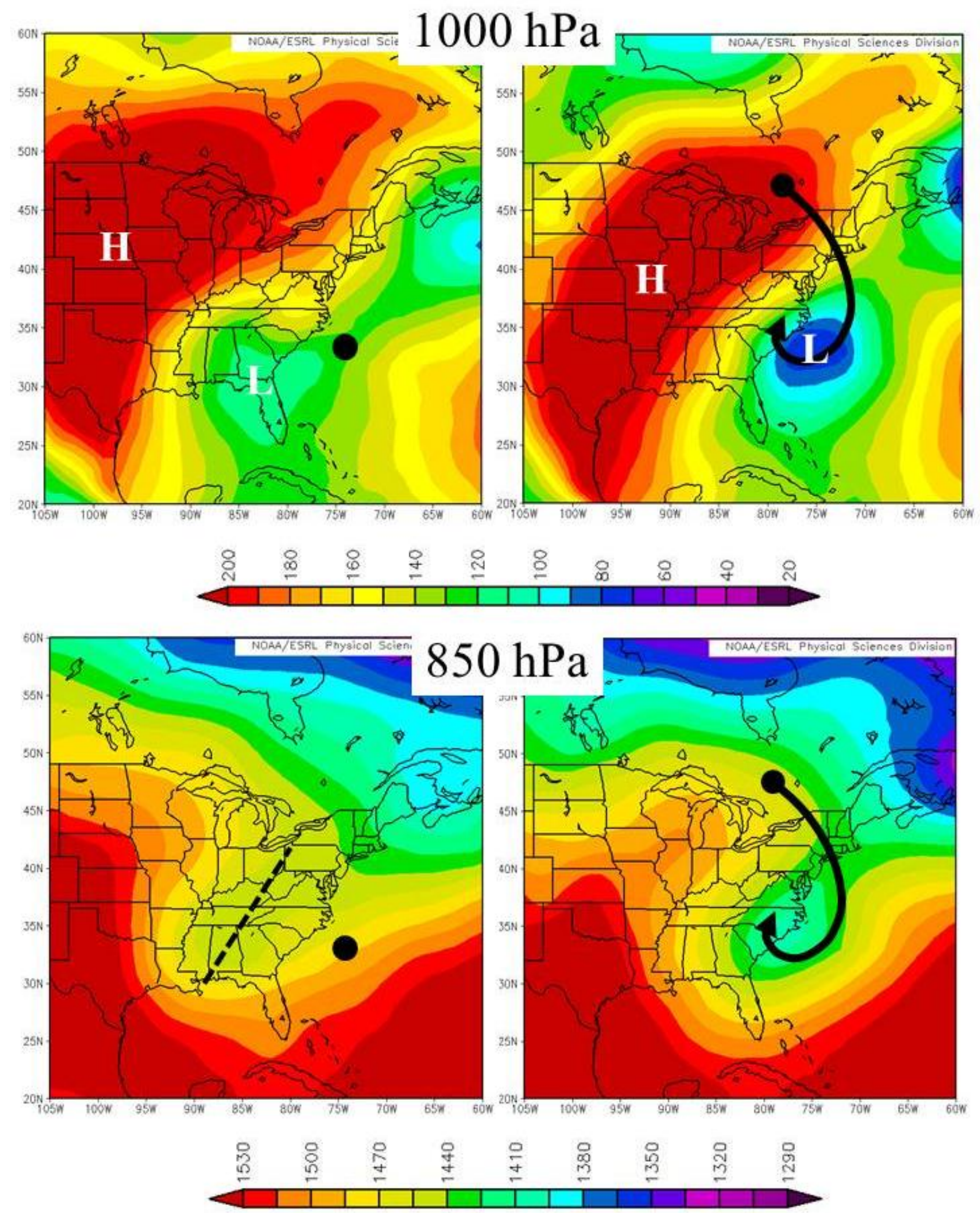

Figure 3.30. Same as Figure 3.29, except for snowstorms with DGZ-cyclonic trajectories. The position of the air parcel at each time step is denoted by a black circle. A sketch of the composite DGZ-cyclonic trajectory is denoted with a solid black line and arrow. 


\section{Chapter 4}

\section{ICE STORMS}

\subsection{Overview}

This chapter describes the results of the back-trajectory and synoptic analyses conducted for seven major ice storms that occurred in central North Carolina from 2000 to 2010. It is organized in a similar manner to Chapter 3, beginning with a discussion of the vertical profiles of temperature, moisture, and vertical velocity at the time and location of heaviest freezing precipitation. This is followed by discussions of the composite trajectories at each of the three critical vertical pressure levels (i.e. near-surface, warm layer zone, and dendritic growth zone) and a set of comparative and ensemble trajectories for a sample of ice storms. The chapter concludes with a discussion of the relationship between the antecedent synoptic-scale circulation and the space-time character of the composite back trajectories.

\subsection{Modeled Vertical Profile}

All of the modeled temperature profiles for ice storms (Table 4.1) revealed an elevated warm layer $\left(>0^{\circ} \mathrm{C}\right.$ ) of varying depth (between 125 and $225 \mathrm{hPa}$ ) and maximum temperature (between 3.0 and $5.9^{\circ} \mathrm{C}$ ). A shallow wedge of subfreezing air (depth between 25 and $100 \mathrm{hPa}$ ) was located beneath the warm layer with minimum temperatures ranging between -1.7 and $-8.0^{\circ} \mathrm{C}$. As discussed in section 2.5.1, modeled soundings during periods of freezing precipitation may overestimate temperatures in the bottom half of the elevated warm 
layer and underestimate temperatures in the surface-based cold wedge. Nevertheless, the temperature profiles for the seven ice storms used in this study revealed above-freezing air in the WLZ and subfreezing air at near-surface level (Table 4.1). Therefore, the trajectory analyses should capture the characteristics of the air associated with the elevated warm layer and surface-based cold wedge. The height of the DGZ ranged from 600 to $450 \mathrm{hPa}$, which was slightly higher than the heights observed in the snowstorm cases. In general, Table 4.1 revealed that there can be much variability in the depths and temperatures of the elevated warm layer and cold wedge during ice storms and that reliance on partial thicknesses (i.e. 1000-850 and 850-700 $\mathrm{hPa}$ ) may bias a precipitation-type forecast.

Analysis of modeled moisture profiles (Table 4.2) revealed high levels of water vapor (generally 3 to $6 \mathrm{~g} / \mathrm{kg}$ ) through the lower troposphere with maximum moisture contents (5.2 to $6.8 \mathrm{~g} / \mathrm{kg}$ ) in the elevated warm layer. These values are more than double the moisture contents observed in the snowstorm cases. The higher moisture contents in the ice storm cases may be tied to the stronger advection of warm, moist air and higher saturation mixing ratios connected with warmer WLZ temperatures. As in the snowstorm cases, a decrease in moisture content was observed below the elevated warm layer and was likely tied to the advection of dry air from an upstream anticyclone (i.e. cold-air damming). Moisture contents in the DGZ were between 1.8 and $2.1 \mathrm{~g} / \mathrm{kg}$, consistent with observations from the snowstorm cases.

Analyses of modeled mean instantaneous vertical velocity (Table 4.3) revealed moderate to strong lift in the DGZ (-4.9 to $-12.6 \mathrm{~cm} / \mathrm{s}$ ) with only two profiles exhibiting vertical velocities of at least $-10 \mathrm{~cm} / \mathrm{s}$ in the DGZ. These velocities were weaker compared to those observed in the snowstorm cases and are indicative of isentropic lifting that typically 
occurs over warm or quasi-stationary frontal boundaries. None of the seven profiles exhibited maximum lifting in the DGZ. In five of the seven profiles, the level of maximum lifting occurred above the DGZ. This type of profile has been tied to the formation of smaller hydrometeors (i.e. high snow-liquid ratio; J. Cortinas, personal communication, 2006). As these hydrometeors fall through the elevated warm layer and melt, they absorb less latent heat compared to larger hydrometeors, thereby helping to limit the erosion of the elevated warm layer through diabatic cooling and increase the duration of freezing precipitation. 
Table 4.1. Modeled vertical temperature $\left({ }^{\circ} \mathrm{C}\right)$ profile for each ice storm. Temperatures associated with the dendritic growth zone (warm layer zone $>0^{\circ} \mathrm{C}$ ) are highlighted in gray (red). The maximum temperature in the warm layer zone is underlined.

\begin{tabular}{|c|c|c|c|c|c|c|c|}
\hline $\begin{array}{l}\text { Pressure } \\
(\mathrm{hPa})\end{array}$ & 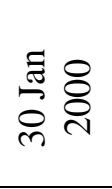 & 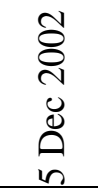 & 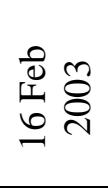 & 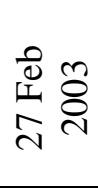 & 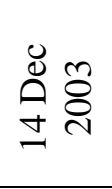 & 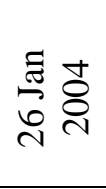 & 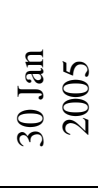 \\
\hline 300 & -49.7 & -41.5 & -42.0 & -44.5 & -42.1 & -41.7 & -50.6 \\
\hline 350 & -40.7 & -30.7 & -34.0 & -37.2 & -34.2 & -32.6 & -38.2 \\
\hline 400 & -32.7 & -24.6 & -26.0 & -30.0 & -27.2 & -25.6 & -36.2 \\
\hline 450 & -25.8 & -16.3 & -19.4 & -22.1 & -20.7 & -19.6 & -25.9 \\
\hline 500 & -19.7 & -13.7 & -14.8 & -18.9 & -16.1 & -14.5 & -22.4 \\
\hline 550 & -14.1 & -9.6 & -9.6 & -14.7 & -11.4 & -10.2 & -17.1 \\
\hline 600 & -9.3 & -6.4 & -6.2 & -11.4 & -8.5 & -7.5 & -13.0 \\
\hline 650 & -6.0 & -3.6 & -3.3 & -7.8 & -5.6 & -4.5 & -9.2 \\
\hline 700 & -2.5 & -0.5 & -0.9 & -3.9 & -2.4 & -2.4 & -5.4 \\
\hline 725 & -0.9 & 1.2 & -0.3 & -2.1 & -0.8 & -1.4 & -3.7 \\
\hline 750 & 0.6 & 2.6 & 1.2 & -0.6 & 0.5 & -0.4 & -2.1 \\
\hline 775 & 1.3 & 3.2 & 2.7 & 0.9 & 1.3 & 0.2 & -1.5 \\
\hline 800 & 2.4 & 4.1 & 3.1 & 2.4 & 2.6 & 1.1 & -0.6 \\
\hline 825 & $\underline{3.0}$ & 4.6 & 5.2 & 3.3 & 3.9 & 2.4 & 0.2 \\
\hline 850 & 2.6 & 4.9 & 5.9 & $\underline{4.1}$ & 4.6 & 3.8 & 1.0 \\
\hline 875 & 2.6 & 4.8 & 2.8 & 4.0 & 5.0 & 4.4 & 2.6 \\
\hline 900 & 1.2 & 3.8 & -1.2 & 2.5 & 3.9 & 2.8 & $\underline{3.7}$ \\
\hline 925 & -0.1 & 2.2 & -5.3 & 1.4 & 1.6 & -0.3 & 3.1 \\
\hline 950 & -1.0 & 0.4 & -8.0 & -0.1 & -0.8 & -4.6 & 0.1 \\
\hline 975 & -2.4 & -1.2 & -7.2 & -3.1 & -1.7 & -5.3 & -3.2 \\
\hline $1000 / \mathrm{Sfc}$ & -2.4 & -2.7 & -6.2 & -3.0 & -1.2 & -3.6 & -3.8 \\
\hline
\end{tabular}


Table 4.2. Same as Table 4.1, except for the modeled vertical moisture profile $(\mathrm{g} / \mathrm{kg})$.

\begin{tabular}{|c|c|c|c|c|c|c|c|}
\hline $\begin{array}{c}\text { Pressure } \\
(\mathrm{hPa})\end{array}$ & 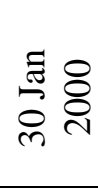 & 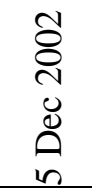 & 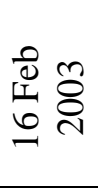 & 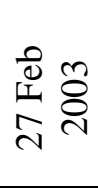 & 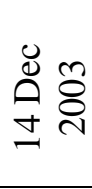 & 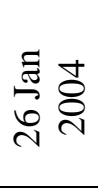 & 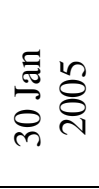 \\
\hline 300 & 0.10 & 0.23 & 0.22 & 0.16 & 0.22 & 0.22 & 0.06 \\
\hline 350 & 0.23 & 0.62 & 0.45 & 0.32 & 0.45 & 0.52 & 0.25 \\
\hline 400 & 0.46 & 1.02 & 0.87 & 0.62 & 0.82 & 0.92 & 0.30 \\
\hline 450 & 0.80 & 1.92 & 1.45 & 1.13 & 1.36 & 1.47 & 0.76 \\
\hline 500 & 1.28 & 2.31 & 2.01 & 1.44 & 1.91 & 2.13 & 1.02 \\
\hline 550 & 1.88 & 3.14 & 2.99 & 1.83 & 2.67 & 2.89 & 1.58 \\
\hline 600 & 2.52 & 3.82 & 3.68 & 2.34 & 3.21 & 3.41 & 2.14 \\
\hline 650 & 3.20 & 4.41 & 4.34 & 3.15 & 3.83 & 4.01 & 2.80 \\
\hline 700 & 4.13 & 5.21 & 4.87 & 4.12 & 4.61 & 4.43 & 3.62 \\
\hline 725 & 4.68 & 5.67 & 5.05 & 4.60 & 5.00 & 4.61 & 4.01 \\
\hline 750 & 5.24 & 6.04 & 5.50 & 4.96 & 5.24 & 4.77 & 4.39 \\
\hline 775 & 5.41 & 6.19 & 5.93 & 5.34 & 5.41 & 4.84 & 4.51 \\
\hline 800 & 5.63 & 6.24 & 6.06 & 5.76 & 5.61 & 4.91 & 4.68 \\
\hline 825 & $\underline{5.60}$ & 6.27 & 6.75 & 5.98 & 5.80 & 5.09 & 4.78 \\
\hline 850 & 5.27 & 6.27 & $\underline{6.80}$ & $\underline{6.15}$ & 5.91 & 5.32 & 4.94 \\
\hline 875 & 5.28 & 6.02 & 5.23 & 5.93 & $\underline{5.78}$ & $\underline{5.20}$ & 5.40 \\
\hline 900 & 4.32 & 5.53 & 3.73 & 5.03 & 5.16 & 4.48 & $\underline{5.64}$ \\
\hline 925 & 3.68 & 4.81 & 2.64 & 4.57 & 4.20 & 3.45 & 5.28 \\
\hline 950 & 3.34 & 4.11 & 2.09 & 4.00 & 3.45 & 2.44 & 4.14 \\
\hline 975 & 2.84 & 3.59 & 2.14 & 3.07 & 3.14 & 2.36 & 3.13 \\
\hline $1000 / \mathrm{Sfc}$ & 2.91 & 3.04 & 2.27 & 3.08 & 3.24 & 2.56 & 2.92 \\
\hline
\end{tabular}


Table 4.3. Same as Table 4.1, except for the modeled mean instantaneous vertical velocity $(\mathrm{cm} / \mathrm{s})$. Negative (positive) values indicate upward (downward) vertical motion.

\begin{tabular}{|c|c|c|c|c|c|c|c|}
\hline $\begin{array}{c}\text { Pressure } \\
(\mathrm{hPa})\end{array}$ & 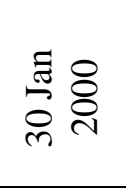 & 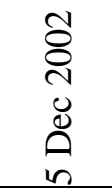 & 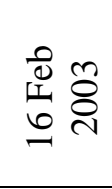 & $\begin{array}{l}\hat{0} \approx \\
\text { ते } \\
\text { ते }\end{array}$ & 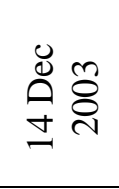 & 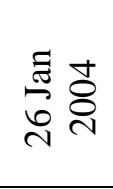 & 疍 \\
\hline 300 & -7.26 & -1.14 & -23.59 & -11.78 & -9.51 & -10.78 & -3.20 \\
\hline 350 & -7.87 & -5.83 & -20.11 & -17.67 & -8.48 & -9.65 & -3.62 \\
\hline 400 & -9.46 & -6.25 & -16.32 & -18.50 & -11.30 & -7.05 & -3.68 \\
\hline 450 & -11.23 & -8.01 & -11.40 & -13.17 & -13.40 & -6.74 & -5.42 \\
\hline 500 & -11.52 & -8.20 & -8.10 & -10.05 & -12.58 & -6.94 & -7.02 \\
\hline 550 & -10.76 & -8.44 & -5.55 & -4.97 & -11.06 & -7.44 & -7.92 \\
\hline 600 & -9.87 & -8.97 & -4.64 & -3.81 & -9.74 & -8.11 & -8.78 \\
\hline 650 & -8.97 & -8.49 & -2.75 & -4.56 & -7.41 & -8.14 & -9.71 \\
\hline 700 & -7.54 & -6.90 & -0.50 & -5.82 & -6.75 & -6.69 & -9.77 \\
\hline 725 & -6.70 & -5.72 & -0.49 & -6.44 & -7.33 & -6.03 & -9.20 \\
\hline 750 & -5.89 & -4.19 & -1.58 & -6.57 & -5.44 & -5.80 & -7.86 \\
\hline 775 & -5.55 & -6.03 & -3.42 & -6.21 & -4.31 & -4.37 & -6.79 \\
\hline 800 & -5.20 & -5.45 & -3.67 & -5.57 & -4.23 & -5.39 & -4.78 \\
\hline 825 & -4.97 & -5.81 & -3.45 & -4.93 & -4.92 & -4.56 & -3.56 \\
\hline 850 & -4.77 & $\underline{-5.10}$ & -3.76 & -4.01 & -4.81 & -3.65 & -3.34 \\
\hline 875 & -3.65 & -4.01 & -3.20 & -3.22 & $\underline{-3.64}$ & -2.63 & -3.71 \\
\hline 900 & -3.05 & -2.51 & -2.58 & -1.63 & -2.81 & -0.90 & -3.86 \\
\hline 925 & -2.29 & -0.68 & -1.96 & -1.69 & -2.56 & -0.22 & -3.39 \\
\hline 950 & -1.99 & -1.19 & -1.24 & -1.60 & -2.33 & 0.13 & -2.48 \\
\hline 975 & -1.39 & -1.11 & -1.03 & -1.10 & -1.69 & -0.21 & -1.52 \\
\hline $1000 / \mathrm{Sfc}$ & -0.13 & -0.58 & -0.63 & -0.69 & -0.81 & -0.48 & -0.69 \\
\hline
\end{tabular}




\subsection{Trajectory Analyses}

\subsubsection{Composite Trajectories}

Figure 4.1 illustrates the mean horizontal component of the 72-hr back trajectories associated with the seven ice storms. The near-surface trajectory originated over Lower Michigan and exhibited anticyclonic curvature as it approached central North Carolina. This trajectory is qualitatively similar to the near-surface trajectory observed in the snowstorm cases. Air parcels terminating in the WLZ also originated over the Great Lakes region, but continued traveling southeastward before turning anticyclonically over the Atlantic Ocean in the vicinity of the Gulf Stream and approaching central North Carolina from the southeast. This is in contrast to the WLZ trajectory observed in the snowstorm cases, which approached central North Carolina from the northeast and traveled over presumably cooler ocean waters. As in the snowstorm cases, the near-surface and WLZ trajectories were similar across all the ice storms, while the DGZ trajectories exhibited two distinct paths and source regions. In four of the seven ice storms, the trajectory terminating in the DGZ originated over the Gulf of Mexico and exhibited general anticyclonic curvature as it approached central North Carolina (hereafter referred to as the "DGZ-Gulf" trajectory; Figure 4.1). In the other three ice storms, the air associated with the DGZ was of eastern Pacific origin and exhibited subtle cyclonic curvature as it approached central North Carolina (hereafter referred to as the "DGZ-Pacific" trajectory; Figure 4.1).

The corresponding mean vertical component of the 72-hr back trajectories is illustrated in Figure 4.2. As in the snowstorm cases, the near-surface air parcel originated at approximately $1 \mathrm{~km}$ above ground level and gradually descended over the course of the trajectory. The WLZ trajectory originated at approximately $1.5 \mathrm{~km}$ and descended to below 
$0.2 \mathrm{~km}$ off of the North Carolina coast before rising again to $1.5 \mathrm{~km}$ over the last 15 hours of the trajectory. The DGZ-Gulf trajectory also originated at approximately $1.5 \mathrm{~km}$ and remained at the same altitude for the first three-quarters of the trajectory before rising to its terminating altitude near $5.5 \mathrm{~km}$. In contrast, the DGZ-Pacific trajectory originated near 5 $\mathrm{km}$ and descended to a minimum height of $3.5 \mathrm{~km}$ at T-30 hours. Beginning at T-6 hours, the DGZ-Pacific trajectory rose from $3.7 \mathrm{~km}$ to its terminating altitude of $5.5 \mathrm{~km}$. These DGZ trajectories reaffirmed the more gradual lift implied in the modeled vertical profile compared to the snowstorm cases.

Figure 4.3 illustrates the air temperature along each trajectory. As in the snowstorm cases, the near-surface air parcels exhibited a gradual warming over the course of the trajectory. However, the starting temperature was colder in the ice storm composite $\left(-18^{\circ} \mathrm{C}\right.$ compared to $-12^{\circ} \mathrm{C}$ ) and exhibited a cooling from -1 to $-4^{\circ} \mathrm{C}$ over the last 12 hours of the trajectory. The WLZ trajectory revealed a gradual warming of air parcels from -18 to $0^{\circ} \mathrm{C}$ over the first two-thirds of the trajectory. However, unlike the snowstorm composite, the WLZ trajectory in the ice storm composite continued to warm to a maximum temperature of $8^{\circ} \mathrm{C}$ at T-12 hours. This was followed by a slight cooling of air parcels to $4^{\circ} \mathrm{C}$ as they entered the elevated warm layer over central North Carolina. Temperatures associated with the DGZ-Pacific trajectory were highly variable over the first third of the trajectory before warming to $0^{\circ} \mathrm{C}$ at $\mathrm{T}-30$ hours. This was followed by a gradual cooling of air parcels as they entered the DGZ. In contrast, air parcels associated with the DGZ-Gulf trajectory remained remarkably warm (near $12^{\circ} \mathrm{C}$ ) for most of the trajectory before abruptly cooling as they entered the DGZ. This temperature trajectory is very similar to the trajectory observed in the DGZ-cyclonic snowstorm composite. 
Figure 4.4 illustrates the moisture composites along each trajectory. As in the snowstorm cases, the moisture content of the near-surface air parcel gradually increased throughout most the trajectory period ( 1 to $2 \mathrm{~g} / \mathrm{kg}$ ) and then increased from 2 to $3 \mathrm{~g} / \mathrm{kg}$ over the last 10 hours of the trajectory. The WLZ trajectory exhibited a small increase in moisture from 0.5 to $1.0 \mathrm{~g} / \mathrm{kg}$ over the first half of the trajectory, but then exhibited an abrupt increase in moisture (1.0 to $6.5 \mathrm{~g} / \mathrm{kg}$ ) from T-32 to T-4 hours. This was followed by a small decrease in moisture content over the last 4 hours of the trajectory. The abrupt increase in moisture corresponded to the position of the WLZ trajectory off of the North Carolina coast at an altitude of less than $0.5 \mathrm{~km}$. This suggests that large evaporative fluxes from the ocean surface contributed to the moisture composite along the WLZ trajectory as it approached the region of heavy freezing precipitation.

In contrast, the DGZ-Pacific trajectory exhibited a relatively weak evaporationprecipitation cycle as moisture contents gradually increased from 0.5 to $3.6 \mathrm{~g} / \mathrm{kg}$ over most of the trajectory period. The decrease in moisture over the last 6 hours of the trajectory was markedly lower in comparison to the DGZ trajectories in the snowstorm composites (i.e. lower rates of condensation and deposition). In contrast, the DGZ-Gulf trajectory exhibited much larger overall moisture contents (4.1 to $6.2 \mathrm{~g} / \mathrm{kg}$ ) than the DGZ-Pacific trajectory. Moreover, the rate decrease in moisture content was much greater with the DGZ-Gulf trajectory, implying higher rates of condensation and deposition. Indeed, ice storms with DGZ-Gulf trajectories exhibited higher maximum precipitation rates $(0.11$ to $0.22 \mathrm{in} / \mathrm{hr})$ than ice storms with DGZ-Pacific trajectories (0.03 to $0.09 \mathrm{in} / \mathrm{hr}$ ).

Composite potential temperature $(\theta)$ values along each trajectory are illustrated in Figure 4.5. As in the snowstorm cases, the near-surface trajectory exhibited very little 
change in $\theta$ over the trajectory period. In contrast, the WLZ trajectory exhibited an increase in $\theta$ over the final 30 hours of the trajectory from 276 to $292 \mathrm{~K}$. The increase in $\theta$ corresponded with a large increase in moisture content along the trajectory (Figure 4.4). In this case, as relatively cold, dry air initially associated with the WLZ trajectory flows out over the warmer Gulf Stream waters, it induces high fluxes of sensible and latent heat from the ocean to the atmosphere (Bane and Osgood 1989). The increase in sensible and latent heat fluxes are therefore reflected in the increase in air parcel temperature and $\theta$ along the WLZ trajectories. The $\theta$ values along the DGZ-Gulf trajectory were nearly constant over the 72-hr trajectory period, while the DGZ-Pacific trajectory exhibited a decrease in $\theta$ during the middle portion of the trajectory. Both DGZ trajectories exhibited increases in $\theta$ over the last 6 hours of the trajectory in response to diabatic heating from condensation and deposition.

Figure 4.6 illustrates the net diabatic contribution to air parcel temperatures along each trajectory. Unlike the snowstorm cases, diabatic processes contributed most significantly to the warming of the WLZ trajectory temperature. Over the 72-hr trajectory period, diabatic processes contributed between 18 and $22^{\circ} \mathrm{C}$ of warming to the WLZ trajectory (i.e. in the absence of diabatic processes, the air parcel temperature by the end of the trajectory would have been 18 to $22^{\circ} \mathrm{C}$ cooler than observed at the beginning of the trajectory). By comparison, diabatic processes contributed approximately $10^{\circ} \mathrm{C}$ of warming to the near-surface trajectory and $12^{\circ} \mathrm{C}$ of warming to the DGZ-Gulf trajectory. In contrast, the DGZ-Pacific trajectory exhibited net diabatic cooling $\left(-5\right.$ to $\left.-11^{\circ} \mathrm{C}\right)$ over the 72 -hr trajectory period. Examination of the observed air parcel temperature and adiabatic air parcel temperature along the WLZ trajectory indicated negligible contributions from diabatic processes over the first half of the trajectory, with significant diabatic warming over the latter 
half of the trajectory (Figure 4.7). In particular, while the observed temperature over the last 6 hours of the trajectory was $4^{\circ} \mathrm{C}$, the adiabatic temperature was $-2^{\circ} \mathrm{C}$. This indicates that the observed temperature would have been below freezing at the time of precipitation in the absence of diabatic warming. With a WLZ below freezing, it is likely that snow would have been observed instead of freezing rain (assuming the near-surface temperature profile was also supportive of snow). 


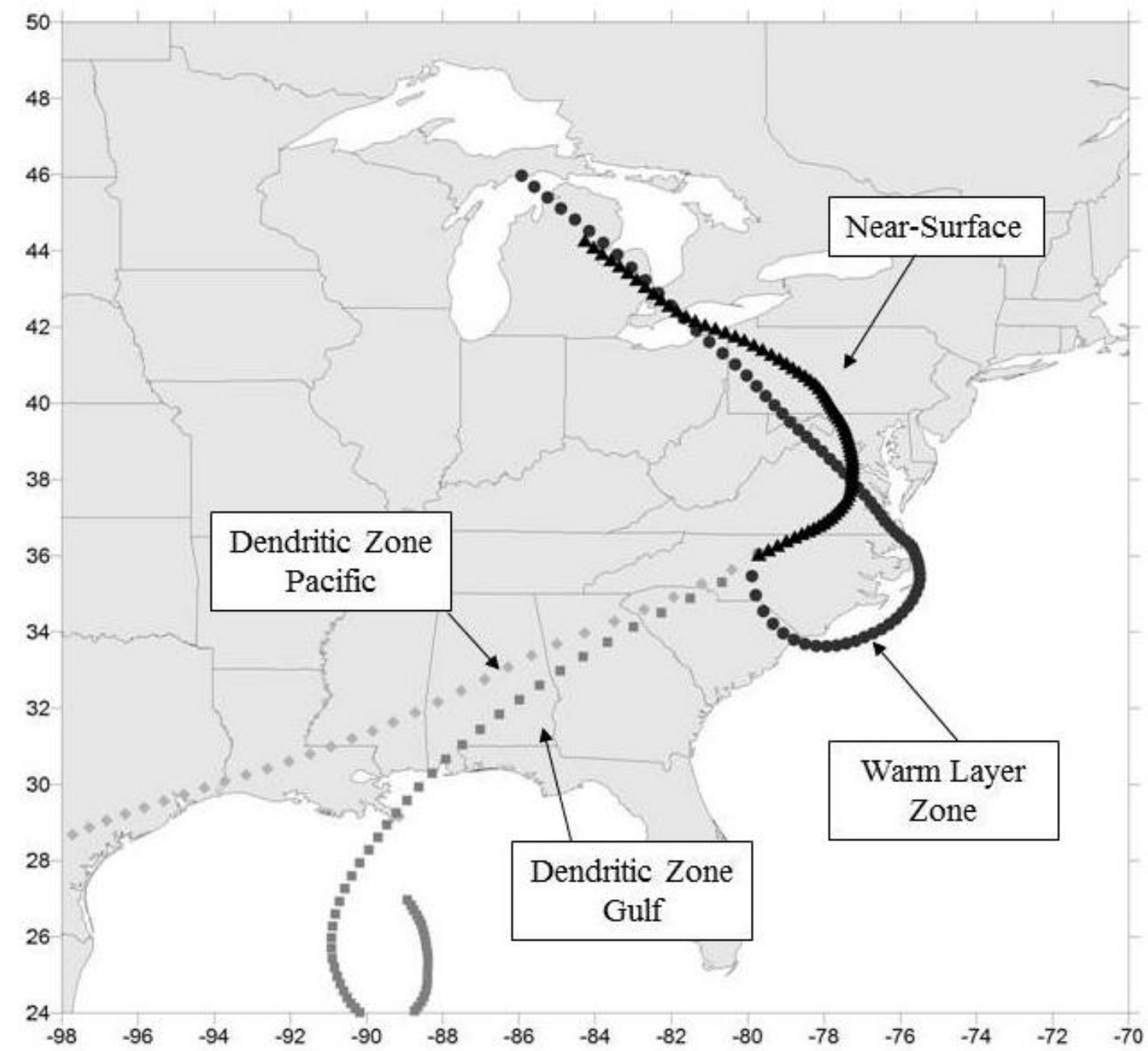

Figure 4.1. Horizontal trajectory composites of air parcels terminating at the near-surface, warm layer zone, and dendritic growth zone in the region of heavy freezing precipitation. 


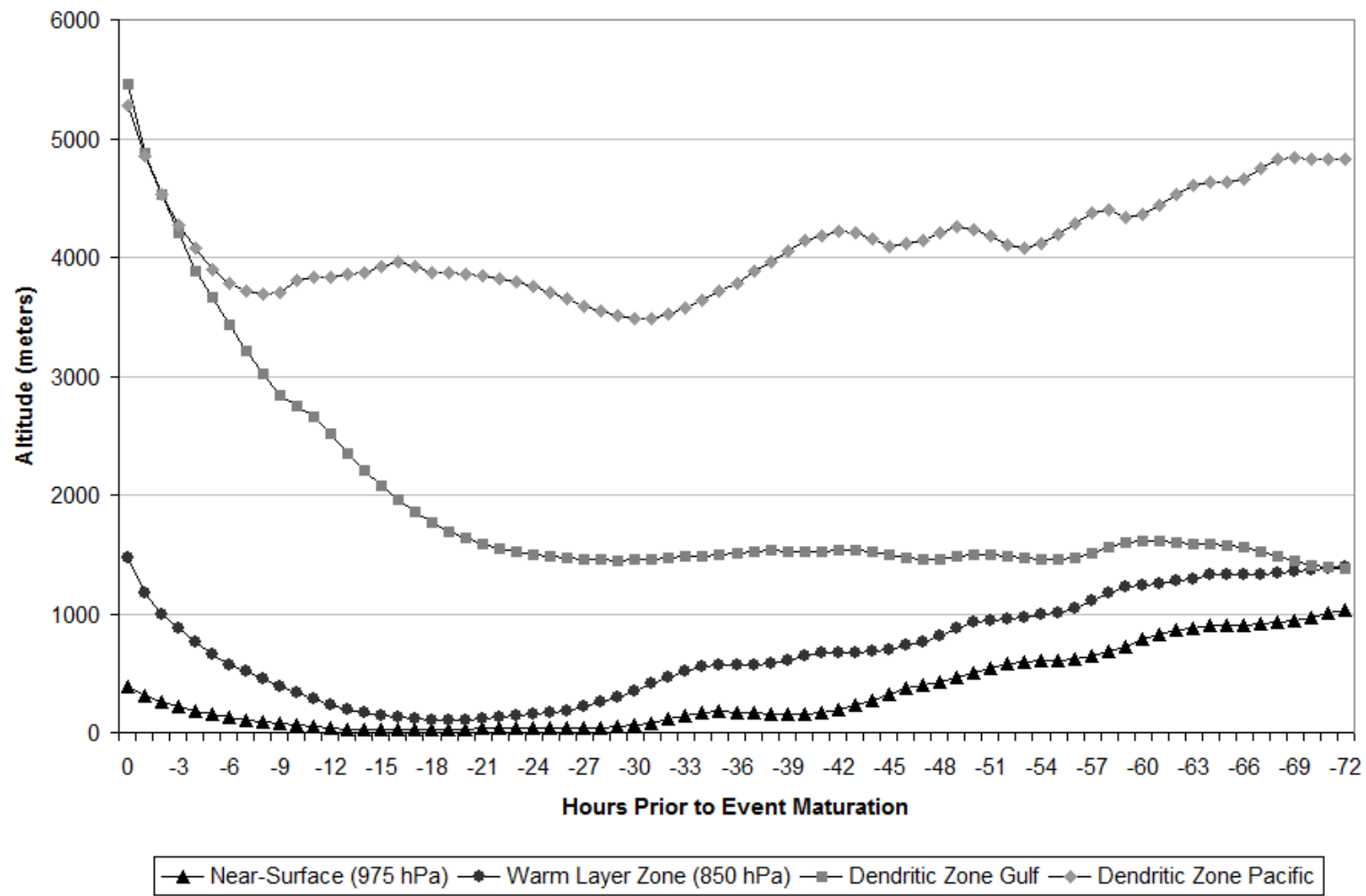

Figure 4.2. Vertical trajectory composites of air parcels terminating at the near-surface, warm layer zone, and dendritic growth zone in the region of heavy freezing precipitation. Composites with range bars can be found in the appendix. 


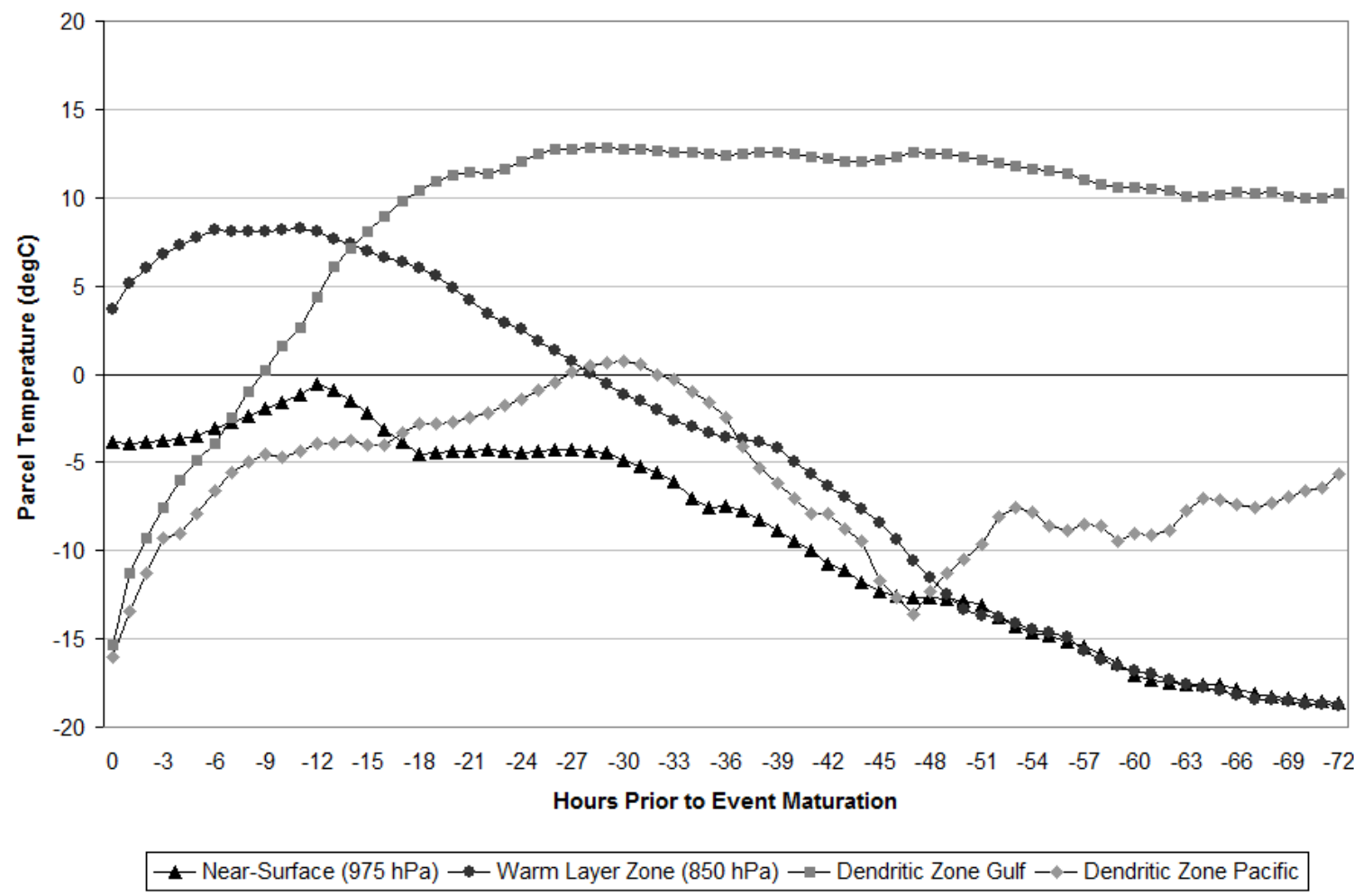

Figure 4.3. Same as Figure 4.2, except for composite trajectories of air parcel temperature. 


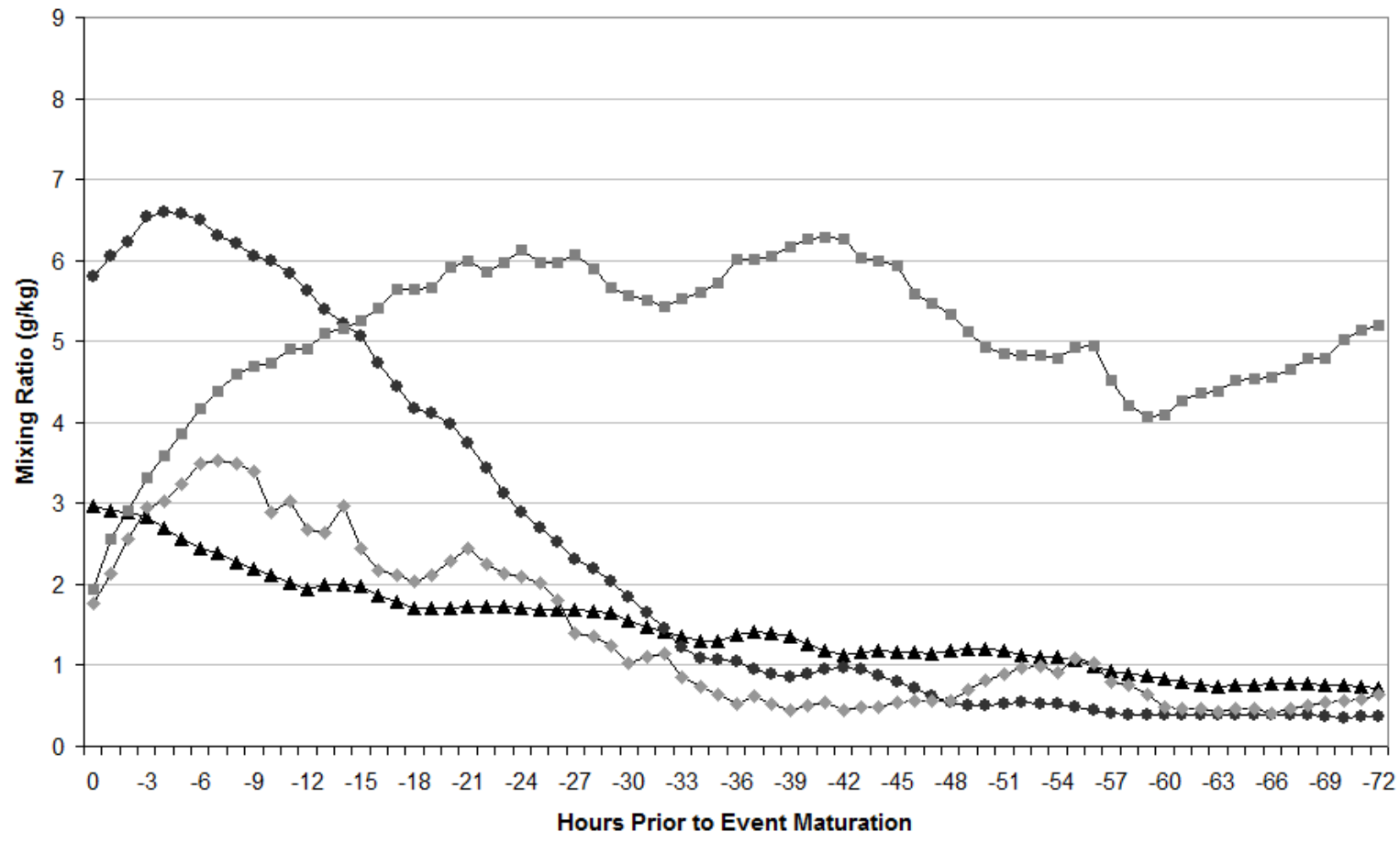

— Near-Surface $(975 \mathrm{hPa})$ - - Warm Layer Zone $(850 \mathrm{hPa})$ - - Dendritic Zone Gulf —४-Dendritic Zone Pacific

Figure 4.4. Same as Figure 4.2, except for moisture composites along each trajectory. 


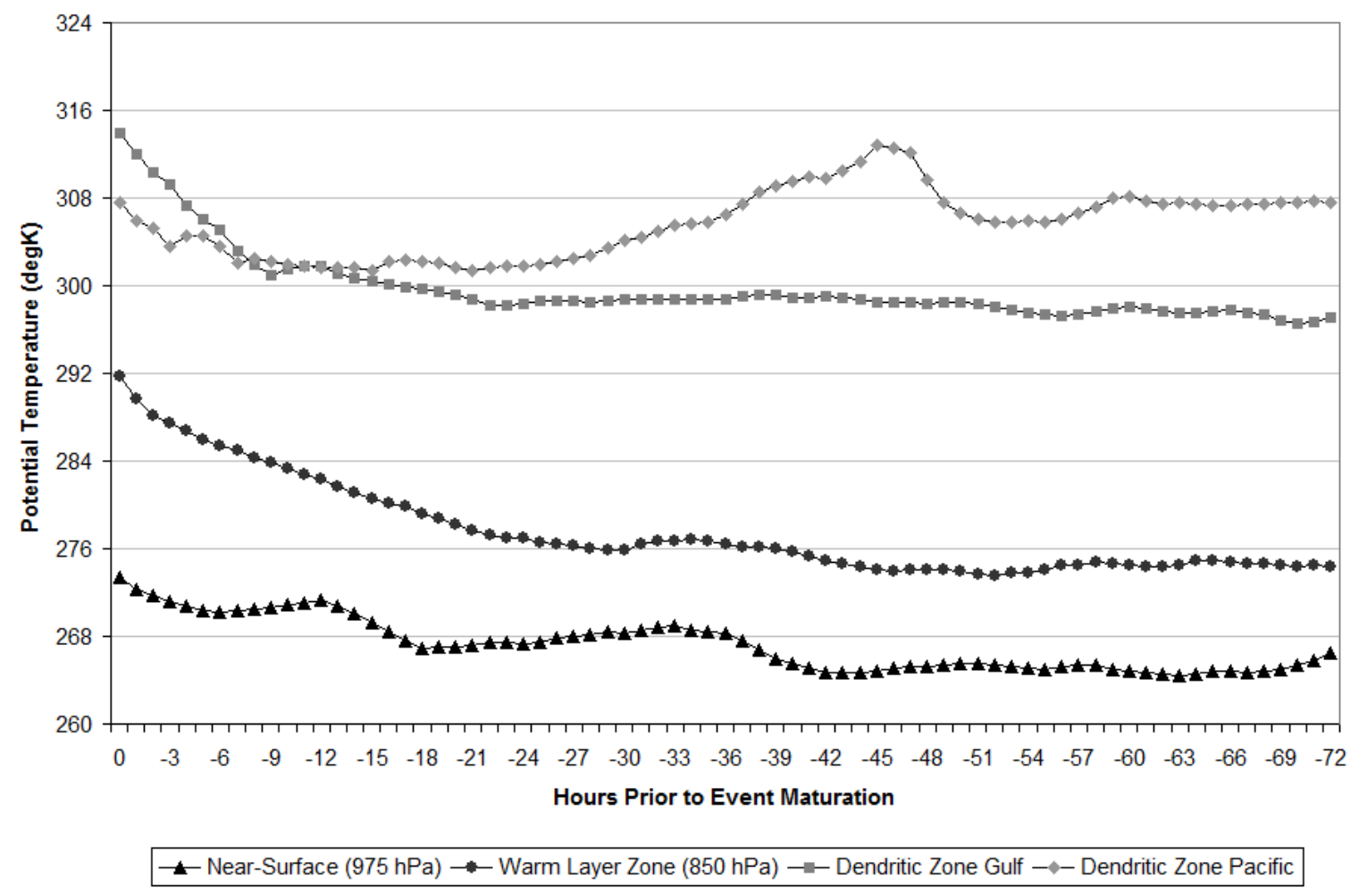

Figure 4.5. Same as Figure 4.2, except for composite trajectories of air parcel potential temperature. 


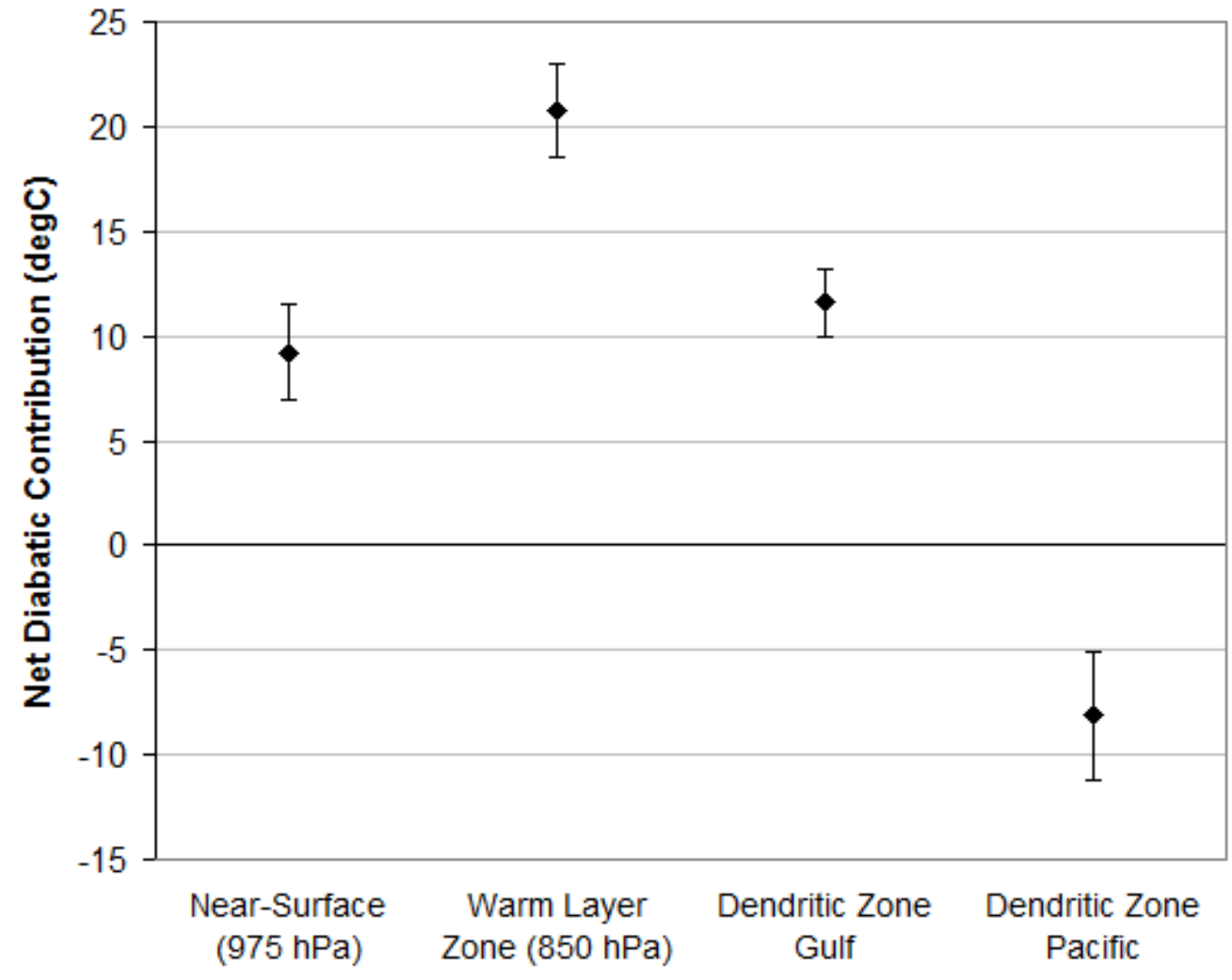

Figure 4.6. Net diabatic contribution to air parcel temperature at each critical level in the region of heavy freezing precipitation. Range bars indicate the maximum and minimum values among all seven ice storms. 


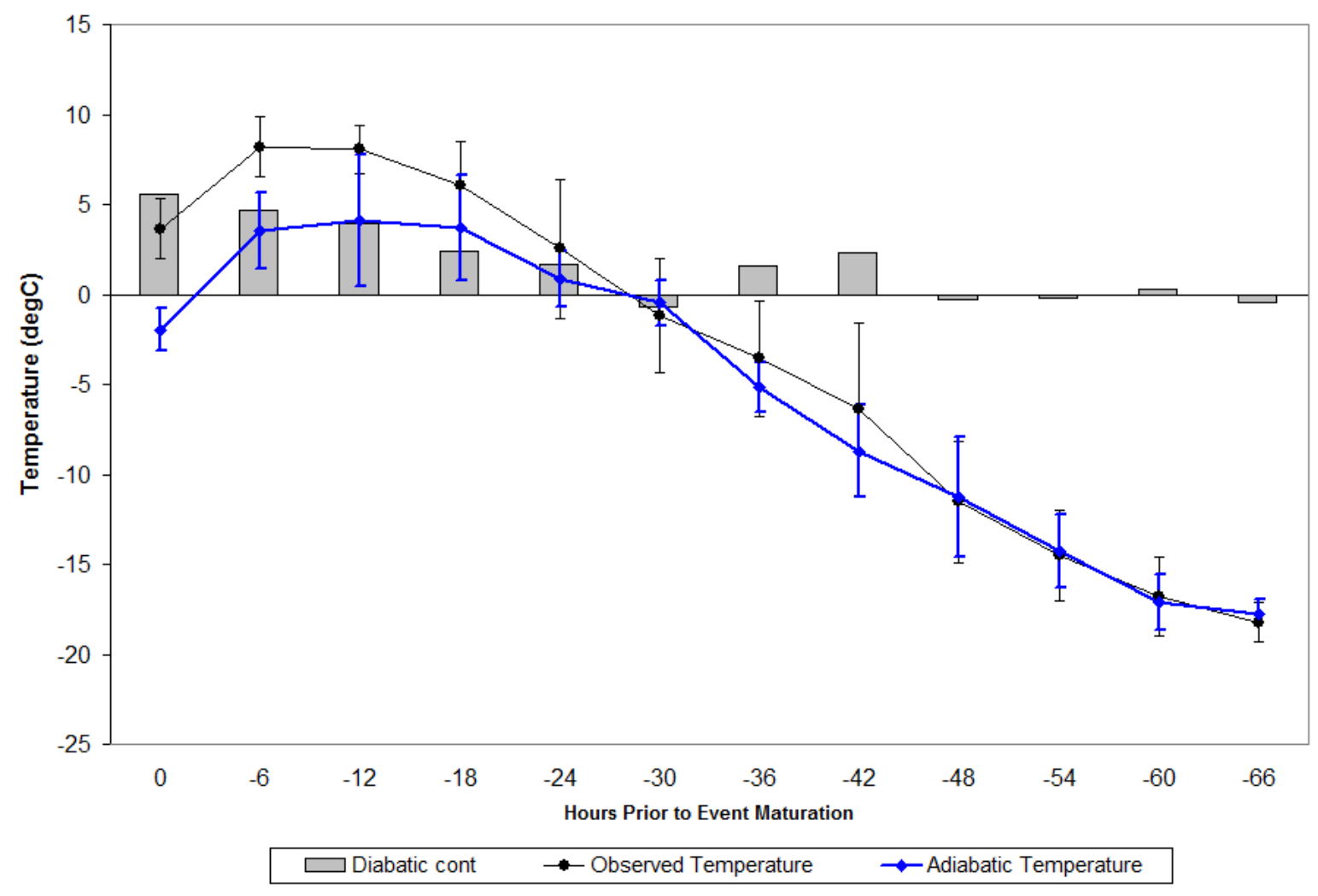

Figure 4.7. Warm layer zone trajectories of observed air parcel temperature, (black line) adiabatic temperature (thick blue line), and diabatic contribution (gray bar) in the region of heavy freezing precipitation at 6-hr trajectory segments. Range bars indicate the maximum and minimum values among all seven ice storms. 


\subsubsection{Comparative and Ensemble Trajectories}

As in Chapter 3, a set of comparative and ensemble trajectories were calculated for two of the seven ice storms to distinguish trajectories at locations with different precipitation types and intensities in a given storm, and to assess how variations in the space-time character of the trajectories, resulting from uncertainties in the model calculations, affect their meteorological properties.

\subsection{2a. Case I: 4-5 December 2002}

The ice storm of 4-5 December 2002 was a major event that initially struck the Southern Plains before moving through the Southeast and mid-Atlantic regions. In North Carolina, the damage was so severe that comparisons were made to Hurricanes Hugo (1989) and Fran (1996) (Jones et al. 2004). Duke Energy referred to the storm as "the worst" in their 100-yr history, as 1.5 million customers lost power across North and South Carolina (Fuhrmann et al. 2009). Freezing rain totals across the Raleigh CWA ranged from 0.12 in $(0.3 \mathrm{~cm})$ in the eastern-most areas of the CWA to over 1.5 in $(3.8 \mathrm{~cm})$ across the central portion of the CWA (see appendix Figure A10). A broad swath of at least 0.5 in $(1.3 \mathrm{~cm})$ of freezing rain extended from southwest to northeast across central North Carolina and included the cities of Charlotte, Greensboro (GSO), and Raleigh-Durham (RDU). However, there was much variation in freezing totals within this area. For example, RDU recorded 1.16 in $(2.9 \mathrm{~cm})$ of freezing rain from the event, with nearly half occurring in a $3-\mathrm{hr}$ period from 04 to 06 UTC 5 December (Table 4.4). In contrast, GSO, located just $100 \mathrm{~km}$ to the west, recorded $0.5 \mathrm{in}(1.3 \mathrm{~cm})$ of freezing rain from the event. Moreover, during the $3-\mathrm{hr}$ period of heavy freezing rain at RDU, only 0.06 in $(0.15 \mathrm{~cm})$ was recorded at GSO. This 
storm was selected for further analysis because of the significant variability in freezing rain totals and precipitation rates across the study area.

Examination of the corresponding back trajectories provides insight into the disparity in precipitation totals between RDU and GSO. Of particular importance are the differences in the space-time character and meteorological properties of the WLZ and DGZ trajectories. At RDU, the DGZ trajectory originated over the western Gulf of Mexico at an altitude of 3.7 $\mathrm{km}$ and traveled northeast (i.e. cyclonically) towards central North Carolina (Figure 4.8a). Air parcels remained above $3.5 \mathrm{~km}$ for most of the trajectory period before ascending into the DGZ at RDU. By comparison, the DGZ trajectory at GSO originated over the eastern Pacific Ocean near the coast of Mexico and took a similar path to the one observed at RDU (Figure 4.8b). Both DGZ trajectories exhibited similar vertical velocities over the last 6 hours of the trajectory $(-7.8 \mathrm{~cm} / \mathrm{s})$, while the DGZ trajectory at GSO remained at a slightly higher altitude $(4 \mathrm{~km})$ for the majority of the trajectory period. Interestingly, despite the small difference in trajectory heights, the maximum moisture content of the DGZ trajectory at GSO was markedly lower $(4.5 \mathrm{~g} / \mathrm{kg})$ than that observed in the DGZ trajectory at RDU $(7.2 \mathrm{~g} / \mathrm{kg})$ (Figure 4.9a). The difference in maximum moisture contents over the evaporationprecipitation cycle likely contributed to the higher rate of freezing rain at RDU.

Inspection of the moisture contents along the WLZ trajectories revealed another possible contributor to the higher freezing rain rate at RDU. Both WLZ trajectories exhibited a rapid rise in moisture content beginning at T-24 hours as they traveled over the Atlantic Ocean (Figure 4.9b). Additionally, air parcels in both WLZ trajectories ascended at a rate of $-4.8 \mathrm{~cm} / \mathrm{s}$ over the last 6 hours of the trajectory. However, the RDU trajectory revealed a decrease in moisture content of approximately $2 \mathrm{~g} / \mathrm{kg}$ over the last 4 hours of the trajectory 
while moisture contents continued to rise along the WLZ trajectory at GSO (Figure 4.9b). The decrease in moisture content in the presence of upward vertical motion indicates that some of the moisture in the WLZ was converted into precipitation at RDU. These results suggest that the WLZ trajectory was an important contributor to the enhanced precipitation rates at RDU.

A set of ensemble trajectories was calculated from RDU for the 4-5 December 2002 ice storm at the time of heaviest freezing rain to test the sensitivity of the back trajectories to changes in the horizontal and vertical offset of the meteorological input data. Figure 4.10 illustrates the space-time character of the near-surface trajectory ensemble members. Nearly all the trajectories originated south of Hudson Bay and exhibited gradual descent over the trajectory period. This sinking motion contributed to adiabatic warming of the air parcels, with nine of the 27 ensemble members exhibiting above-freezing temperatures at T-0 hours (Figure 4.11). Several of these trajectories appeared to travel off the mid-Atlantic coast and therefore may have induced both sensible and latent fluxes. Figure 4.12 illustrates the $\theta$ values along the near-surface trajectory ensembles. Ensemble members with near-surface temperatures above freezing at T-0 hours exhibited a mean increase in $\theta$ over the final 36 hours of the trajectory from approximately $268 \mathrm{~K}$ to $282 \mathrm{~K}$. In contrast, ensemble members with near-surface temperatures below freezing exhibited no net change in $\theta$ over the final 36 hours of the trajectory. This suggests that air parcels that travel over the Atlantic Ocean are diabatically warmed as a result of latent fluxes from the ocean to the atmosphere. The magnitude of these fluxes can have a significant effect on a precipitation-type forecast when the air parcel temperature is near freezing. 
The set of ensemble trajectories terminating in the WLZ is illustrated in Figure 4.13. Nearly all of the trajectories originated over southeast Canada and generally corresponded to the composite WLZ trajectory in Figure 4.1. Several of the ensemble trajectories descended over time and warmed significantly over the trajectory period, in some cases by as much as $50^{\circ} \mathrm{C}$ (Figure 4.14). Interestingly, all 27 ensemble members exhibited above-freezing temperatures in the WLZ at T-0 hours with a range of 1 to $8^{\circ} \mathrm{C}$. The range in WLZ temperatures suggests that in some cases only partial melting of hydrometeors will take place, which could result in mixed precipitation types (Bernstein 2000). As in the composite analysis, the net diabatic contribution to air parcel temperature was calculated for all WLZ ensemble members with comparisons between parcels that were 1 to $4^{\circ} \mathrm{C}$ (i.e. only partial melting likely) and 5 to $8^{\circ} \mathrm{C}$ (i.e. complete melting likely). Figure 4.15 reveals that over the 72-hr trajectory period, ensemble members with WLZ temperatures between 1 and $4^{\circ} \mathrm{C}$ exhibited on average as much as $14^{\circ} \mathrm{C}$ of warming due to diabatic processes (rate of $0.2^{\circ} \mathrm{C} / \mathrm{hr}$ ). In contrast, ensemble members with WLZ temperatures between 5 and $8^{\circ} \mathrm{C}$ exhibited $21^{\circ} \mathrm{C}$ of warming due to diabatic processes (rate of $0.3^{\circ} \mathrm{C} / \mathrm{hr}$ ). Thus, warmer WLZ temperatures were associated with greater rates of diabatic warming over the course of the trajectory period compared to lower WLZ temperatures.

Figure 4.16 illustrates the set of ensemble trajectories terminating in the DGZ. Nine of the 27 ensemble members resembled the composite DGZ-Gulf trajectory and remained below $3 \mathrm{~km}$ for most of the trajectory period (see Figure 4.1). In contrast, the remaining ensemble members more closely resembled the DGZ-Pacific trajectory and generally remained above $3 \mathrm{~km}$ for most of the trajectory period. Differences in the space-time character of these DGZ trajectory types were also reflected in their meteorological properties. 
In particular, maximum moisture contents for ensemble members below $2 \mathrm{~km}$ (i.e. DGZ-Gulf type trajectories) were approximately double the moisture contents observed for ensemble members above $2 \mathrm{~km}$ (i.e. DGZ-Pacific type trajectories), resulting in more moisture available for precipitation. Moreover, the ensemble members with higher moisture contents exhibited well-defined evaporation-precipitation cycles and greater decreases in moisture content over the final 12 hours of the trajectories (i.e. greater rates of condensation and deposition). 
Table 4.4. Hourly precipitation totals (in. liquid equivalent) and present weather for the 4-5 December 2002 ice storm at RDU and GSO. Back trajectories were computed from the time highlighted in boldface.

\begin{tabular}{|c|c|c|c|c|c|}
\hline \multirow{2}{*}{ Date } & \multirow{2}{*}{$\begin{array}{c}\text { Time } \\
\text { (UTC) }\end{array}$} & \multicolumn{2}{|c|}{ RDU } & \multicolumn{2}{|c|}{ GSO } \\
\hline & & $\begin{array}{c}\text { Precip } \\
\text { (Liq. Equiv.) }\end{array}$ & $\begin{array}{c}\text { Present } \\
\text { Weather* }\end{array}$ & $\begin{array}{c}\text { Precip } \\
\text { (Liq. Equiv.) }\end{array}$ & $\begin{array}{c}\text { Present } \\
\text { Weather* }\end{array}$ \\
\hline $12 / 04 / 2002$ & 18 & & & $\operatorname{Tr}$ & -SN BR \\
\hline $12 / 04 / 2002$ & 19 & & & 0.04 & -SN BR \\
\hline $12 / 04 / 2002$ & 20 & 0.03 & $-\mathrm{SN}$ & 0.04 & SN FZFG \\
\hline $12 / 04 / 2002$ & 21 & 0.10 & -SN BR & 0.04 & -SN BR \\
\hline $12 / 04 / 2002$ & 22 & 0.04 & & 0.04 & $-\mathrm{SN}$ \\
\hline $12 / 04 / 2002$ & 23 & 0.10 & FZRA & 0.02 & -SN BR \\
\hline $12 / 05 / 2002$ & 00 & 0.08 & FZRA BR & 0.03 & -SN BR \\
\hline $12 / 05 / 2002$ & 01 & 0.06 & -FZRA BR & 0.03 & $-\mathrm{SN}$ \\
\hline $12 / 05 / 2002$ & 02 & 0.02 & -FZRA BR & 0.03 & -FZRA \\
\hline $12 / 05 / 2002$ & 03 & 0.05 & FZRA BR & 0.03 & -FZRA \\
\hline $12 / 05 / 2002$ & 04 & 0.13 & FZRA BR & 0.03 & -FZRA \\
\hline $12 / 05 / 2002$ & 05 & 0.16 & FZRA BR & $\operatorname{Tr}$ & -FZRA \\
\hline $12 / 05 / 2002$ & 06 & 0.21 & FZRA BR & 0.03 & -FZRA \\
\hline $12 / 05 / 2002$ & 07 & 0.08 & -FZRA BR & 0.05 & FZRA \\
\hline $12 / 05 / 2002$ & 08 & 0.06 & -FZRA BR & 0.06 & FZRA BR \\
\hline $12 / 05 / 2002$ & 09 & 0.05 & -FZRA BR & 0.08 & -FZRA \\
\hline $12 / 05 / 2002$ & 10 & 0.02 & -FZRA BR & 0.07 & -FZRA \\
\hline $12 / 05 / 2002$ & 11 & 0.09 & FZRA BR & 0.04 & FZRA BR \\
\hline $12 / 05 / 2002$ & 12 & 0.03 & -FZRA BR & 0.05 & FZRA BR \\
\hline $12 / 05 / 2002$ & 13 & 0.02 & -FZRA BR & & \\
\hline
\end{tabular}

$* \mathrm{RA}=$ rain $; \mathrm{BR}=$ mist $; \mathrm{SN}=$ snow $; \mathrm{FZRA}=$ freezing rain $; \mathrm{FZFG}=$ freezing fog 

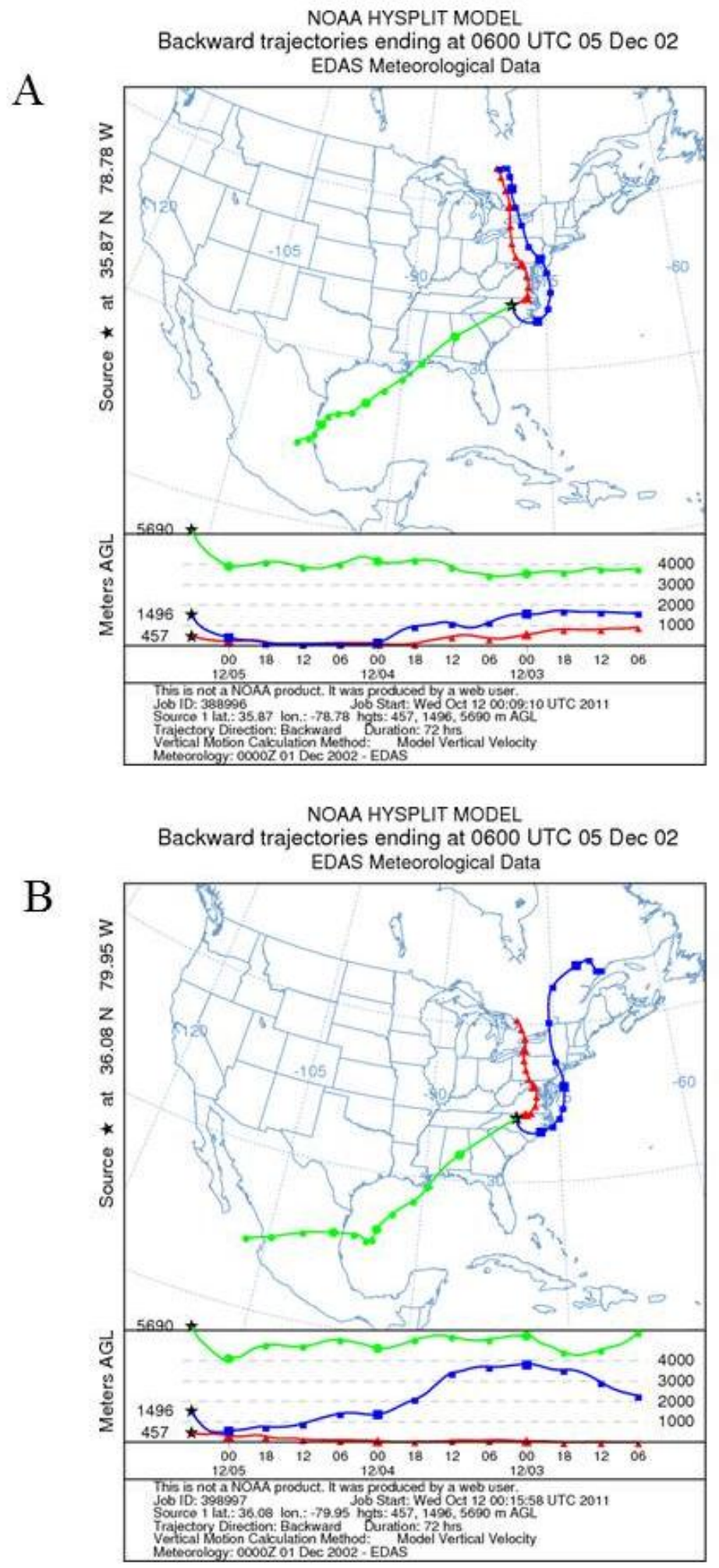

Figure 4.8. Horizontal and vertical trajectories for 06 UTC 5 December 2002 terminating at the near-surface level (red trajectories), WLZ (blue trajectories), and DGZ (green trajectories) at (A) Raleigh-Durham (RDU) and (B) Greensboro (GSO). 
(A) Dendritic Growth Zone

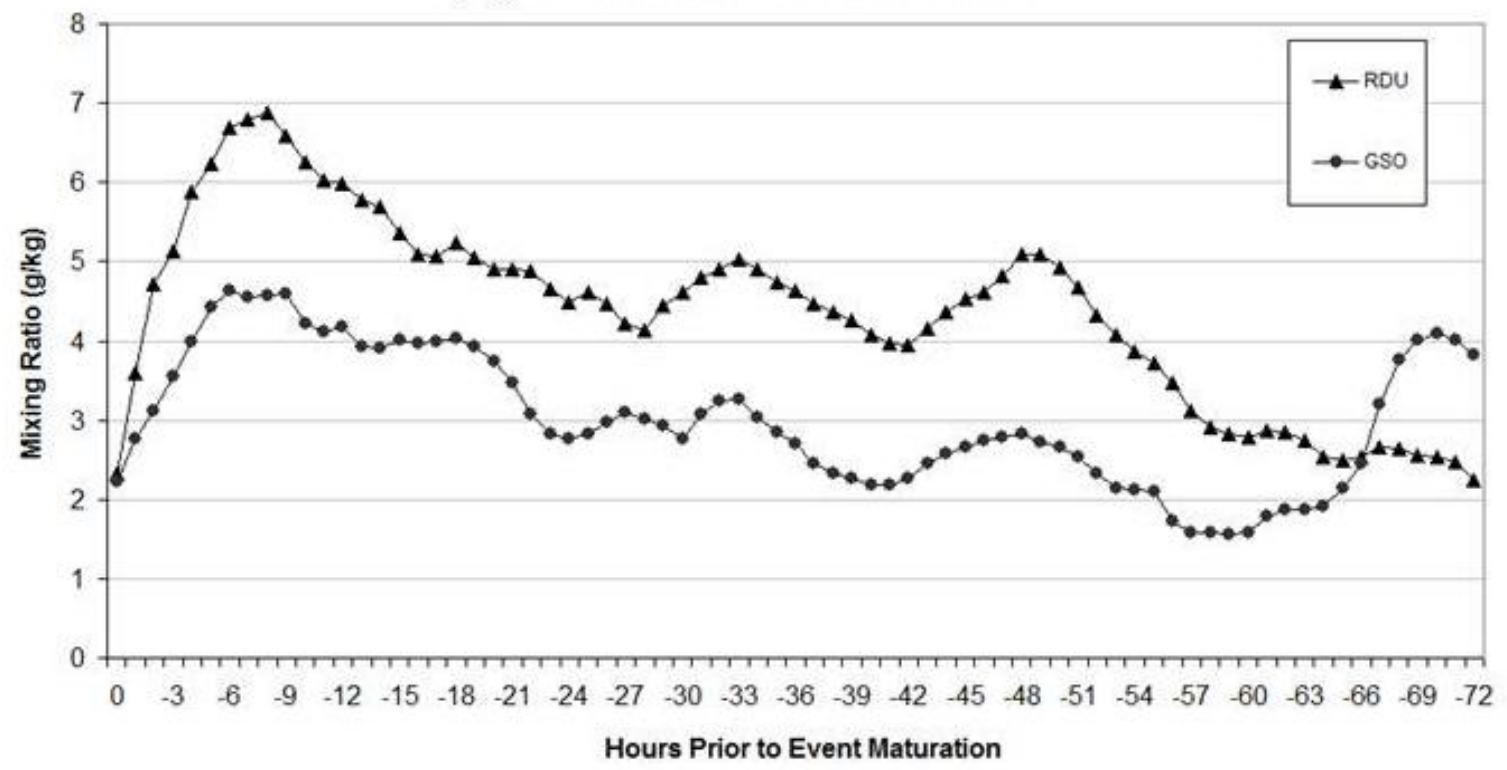

(B) Warm Layer Zone

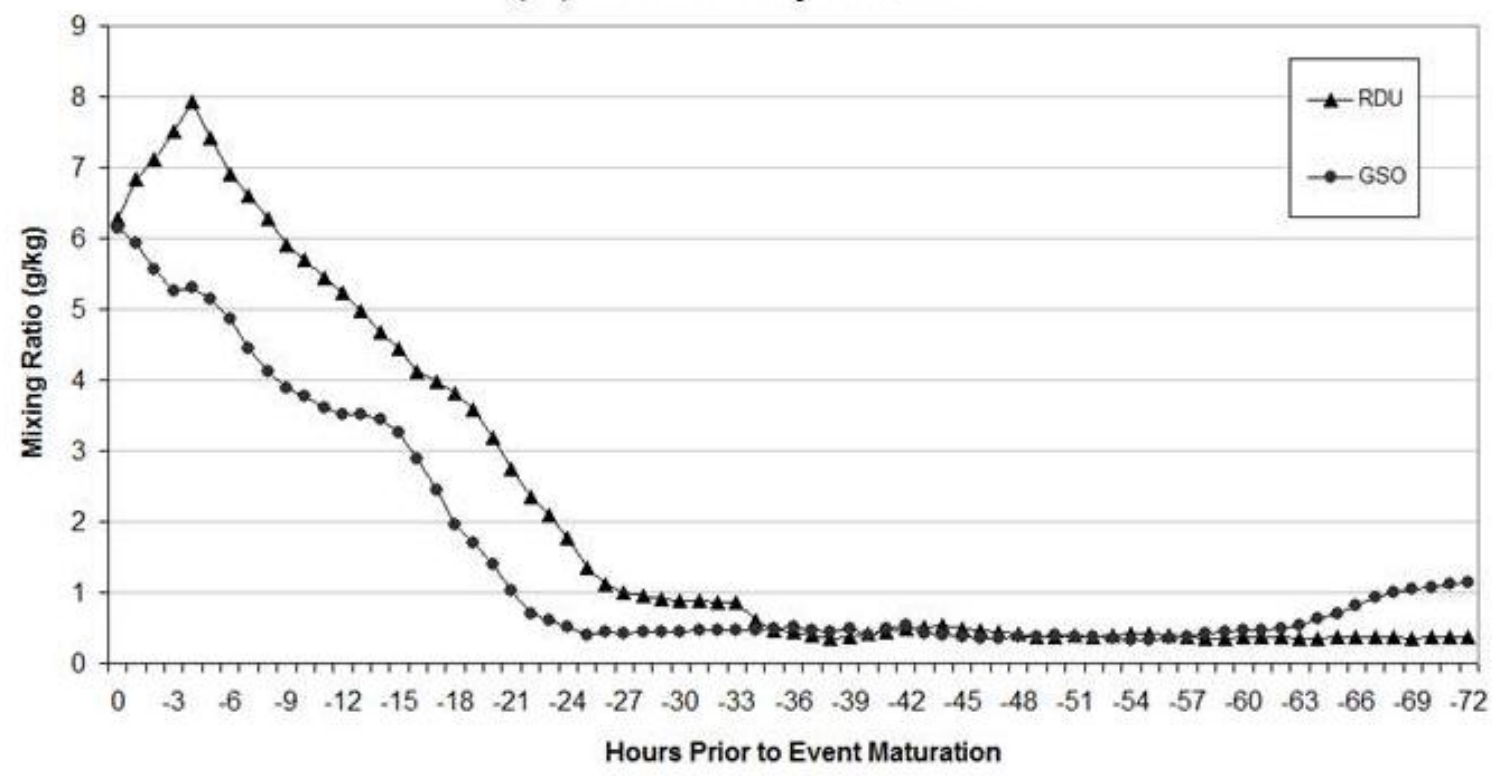

Figure 4.9. Air parcel trajectories of moisture content for 06 UTC 5 December 2002 terminating in the (A) dendritic growth zone and (B) warm layer zone at RDU and GSO. 


\section{NOAA HYSPLIT MODEL Backward trajectories ending at 0600 UTC 05 Dec 02 EDAS Meteorological Data}

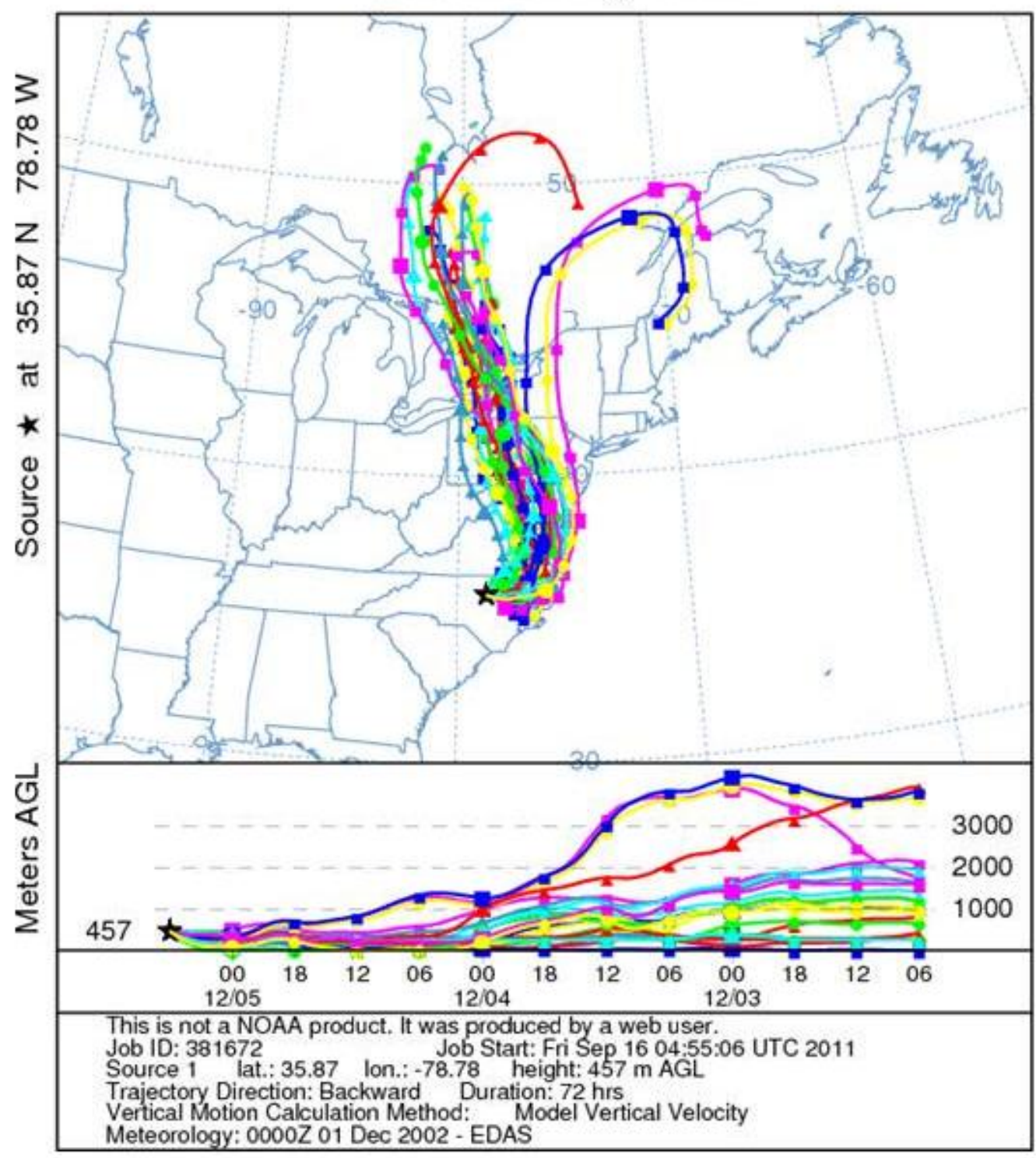

Figure 4.10. Backward ensemble trajectories for 06 UTC 5 December 2002 calculated from RDU at the near-surface level. Vertical trajectories are shown in the bottom panel. 


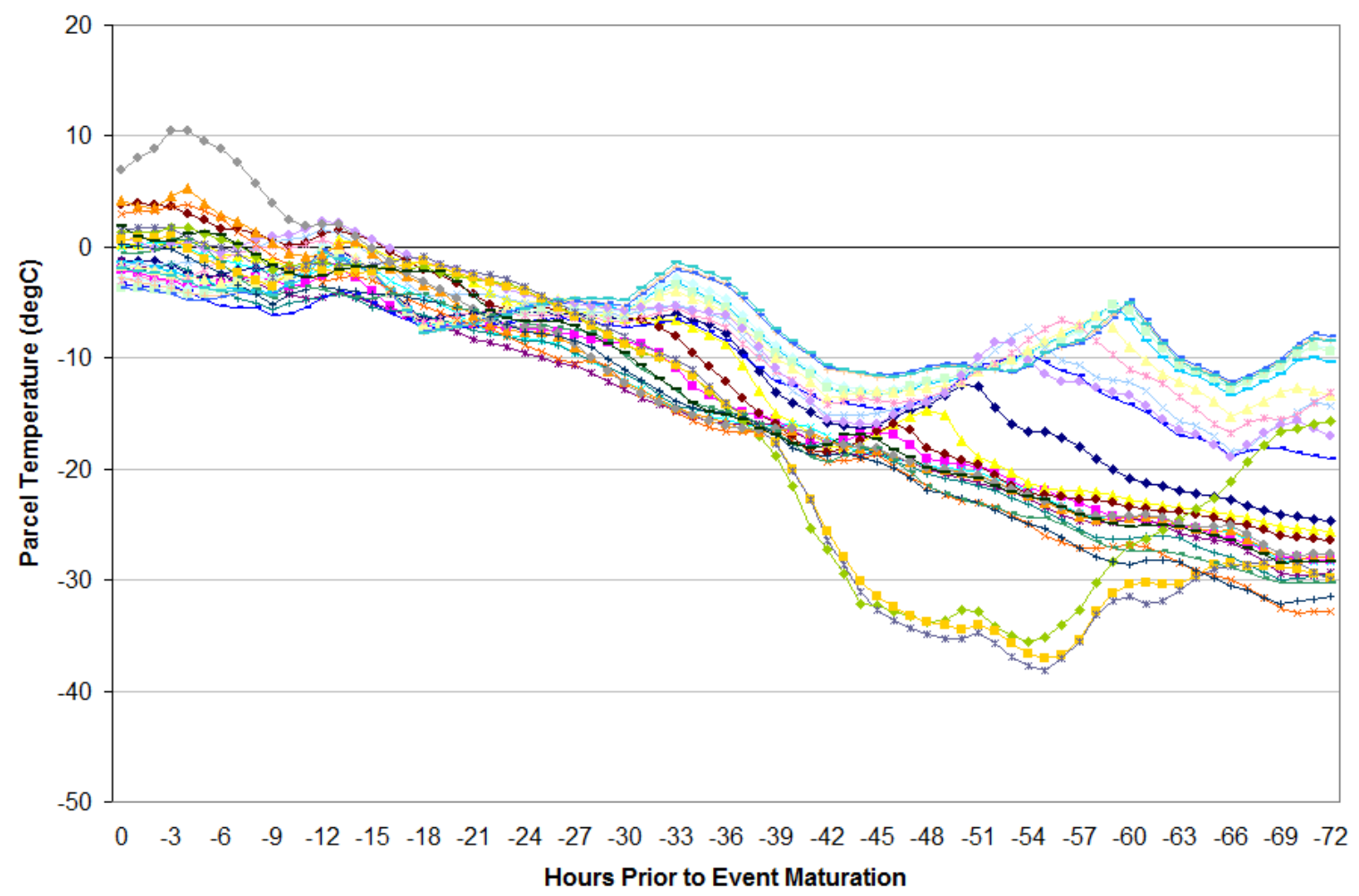

Figure 4.11. Backward ensemble temperature trajectories for 06 UTC 5 December 2002 calculated from RDU at the near-surface level. 


\section{(A) Members with $975 \mathrm{hPa} \mathbf{T}>\mathbf{0}$ degC}

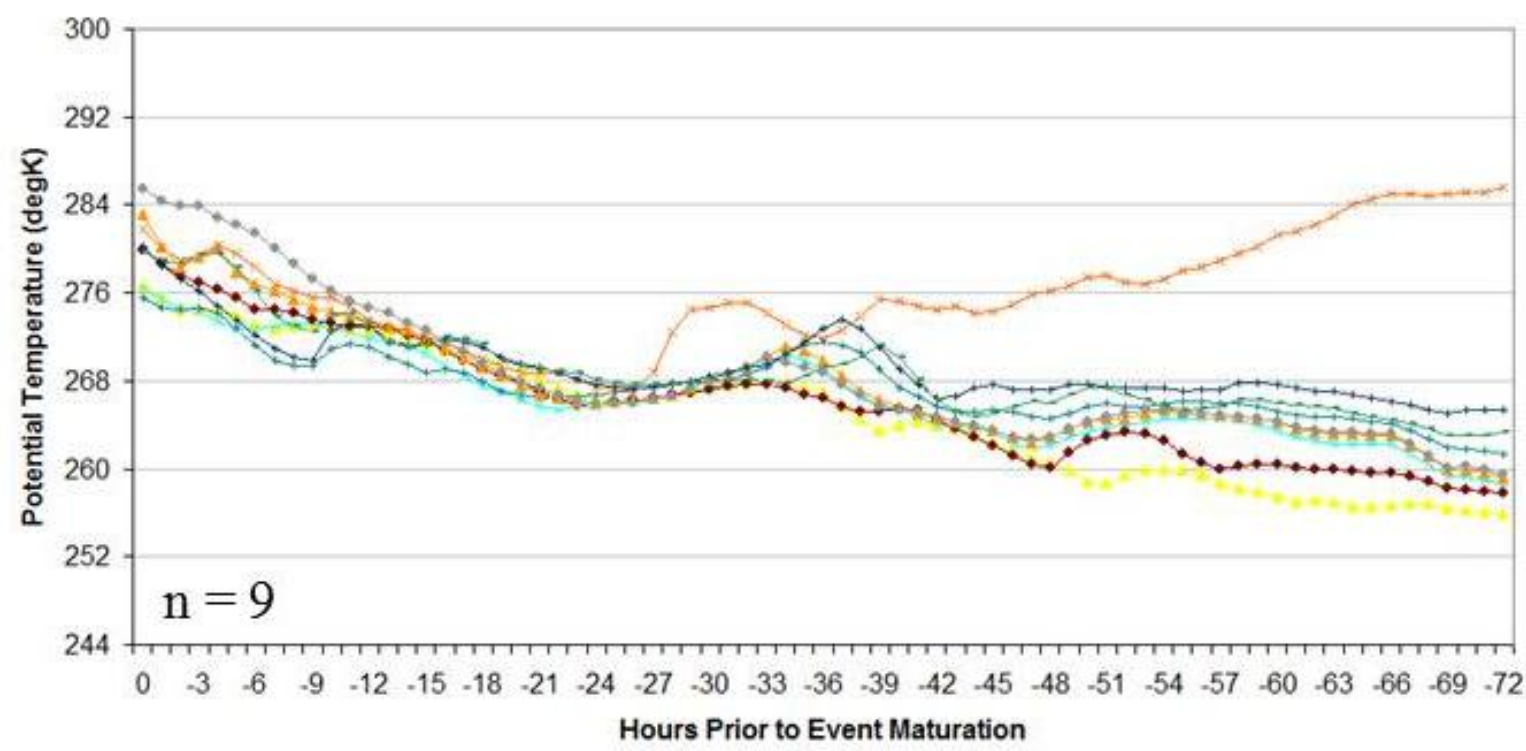

(B) Members with $975 \mathrm{hPa} \mathbf{T}<\mathbf{0}$ degC

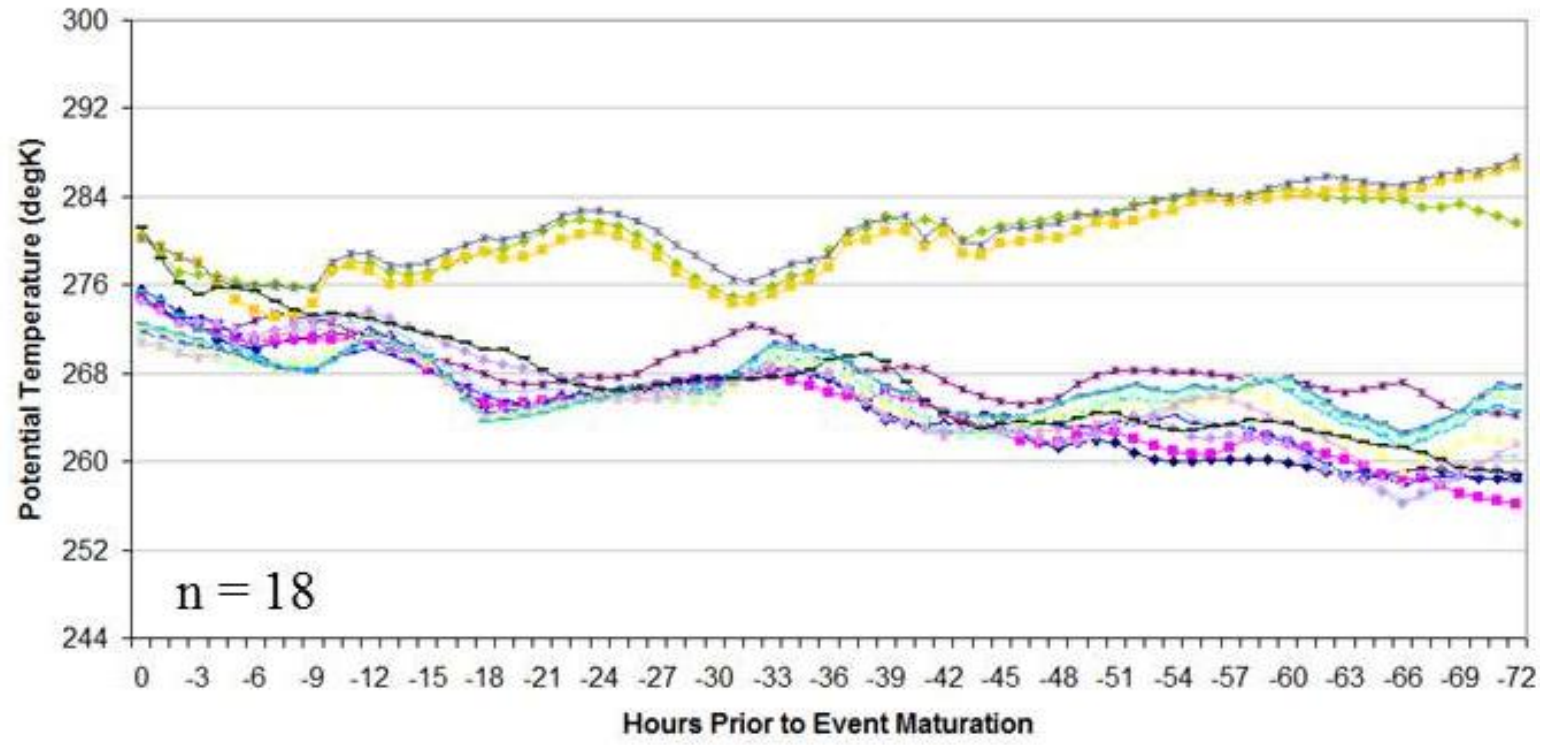

Figure 4.12. Backward ensemble trajectories of potential temperature for 06 UTC 5 December 2002 calculated from RDU with near-surface trajectories (A) above freezing and (B) below freezing. 


\section{NOAA HYSPLIT MODEL \\ Backward trajectories ending at 0600 UTC 05 Dec 02 \\ EDAS Meteorological Data}

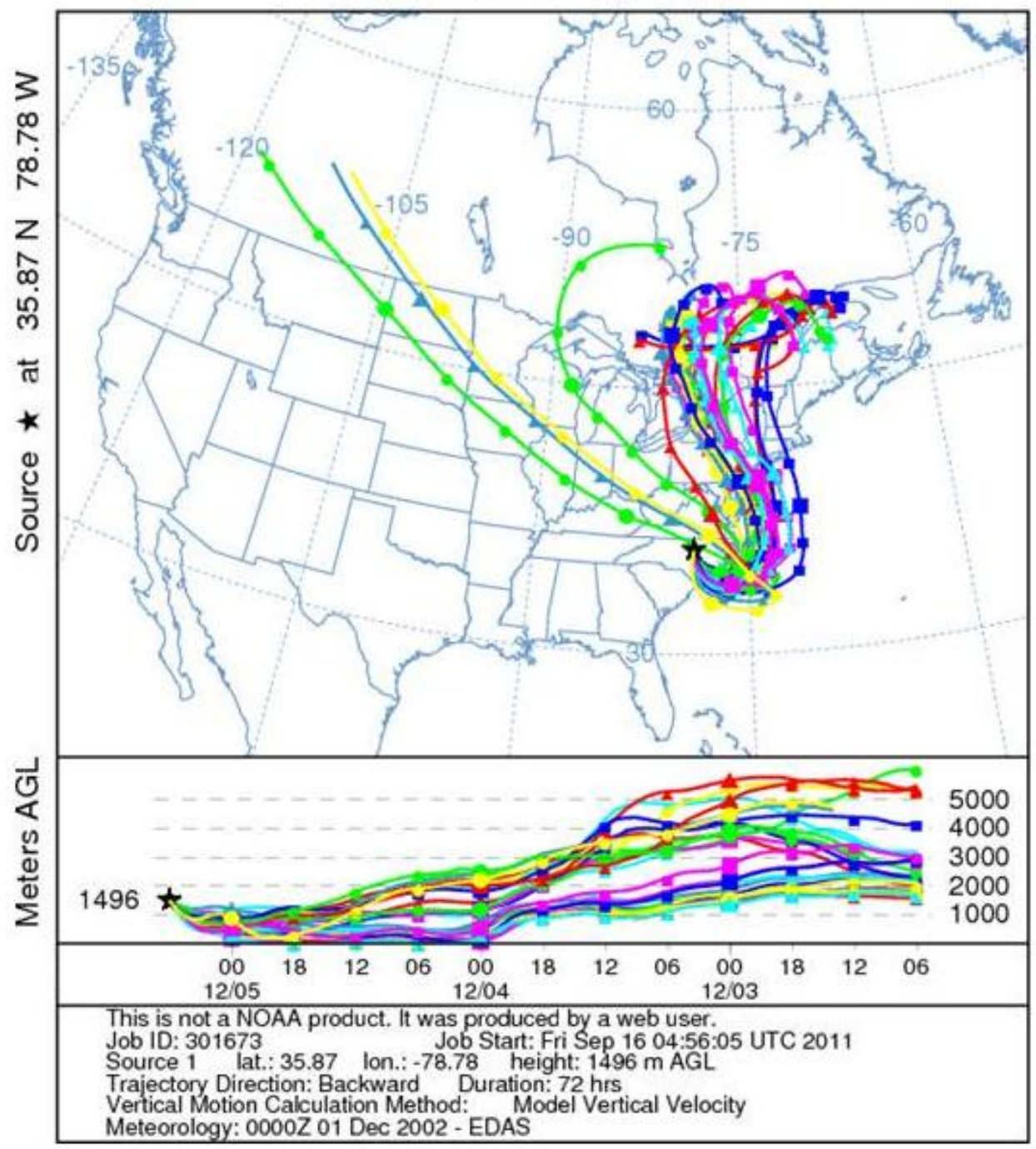

Figure 4.13. Same as Figure 4.10, except at the WLZ. 


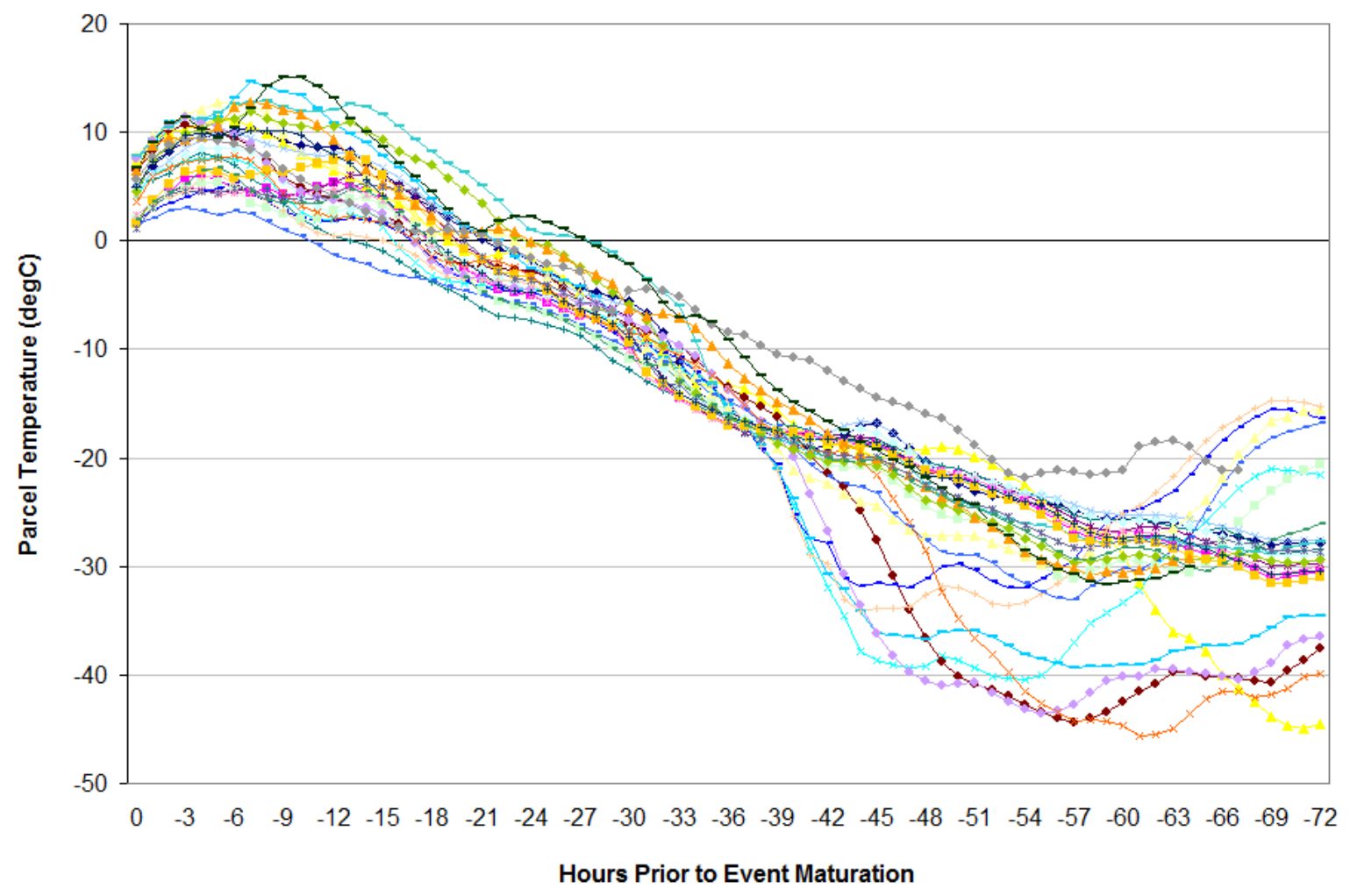

Figure 4.14. Same as Figure 4.11, except at the WLZ. 
(A) Members with $850 \mathrm{hPa}$ T 5-8 degC

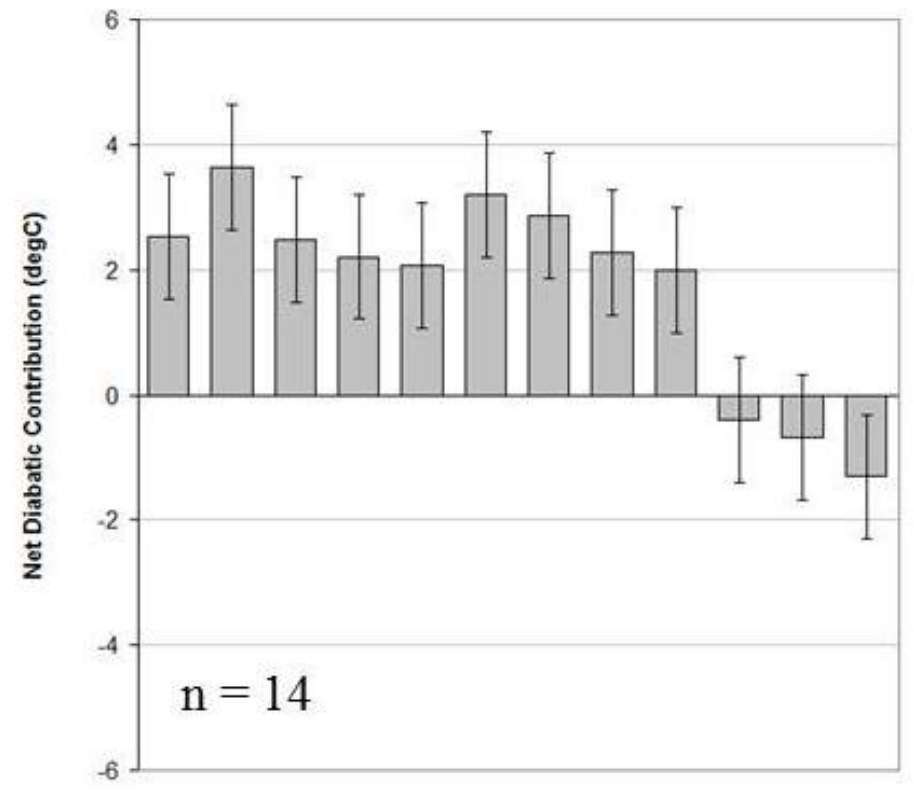

(B) Members with $850 \mathrm{hPa}$ T 1-4 degC

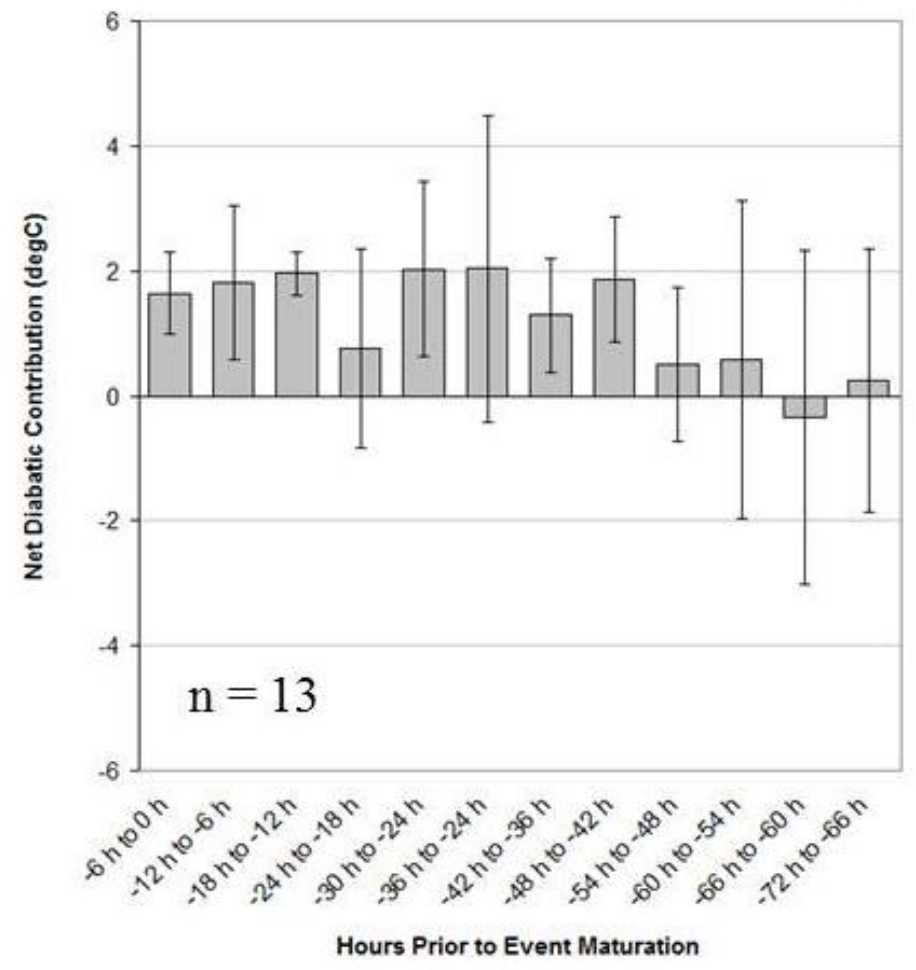

Figure 4.15. Net diabatic contribution to air parcel temperature trajectories at RDU with WLZ temperatures (A) between 5 and $8^{\circ} \mathrm{C}$ and (B) between 1 and $4^{\circ} \mathrm{C}$ at 6 -hr trajectory segments. Range bars indicate the maximum and minimum values among the ensemble members. 


\section{NOAA HYSPLIT MODEL Backward trajectories ending at 0600 UTC 05 Dec 02 EDAS Meteorological Data}

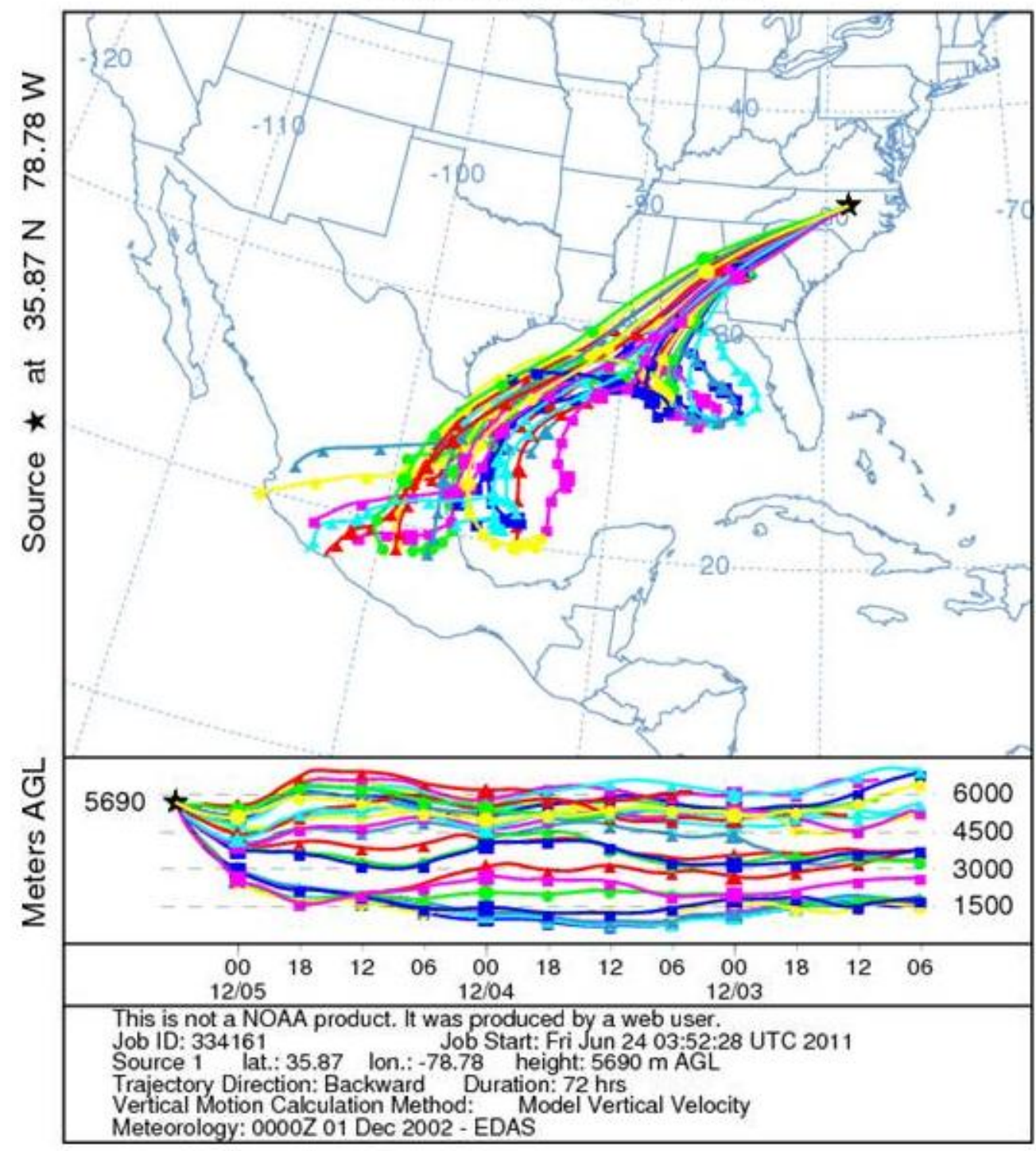

Figure 4.16. Same as Figure 4.10, except at the DGZ. 


\section{(A) Members $<\mathbf{2}$ km AGL}

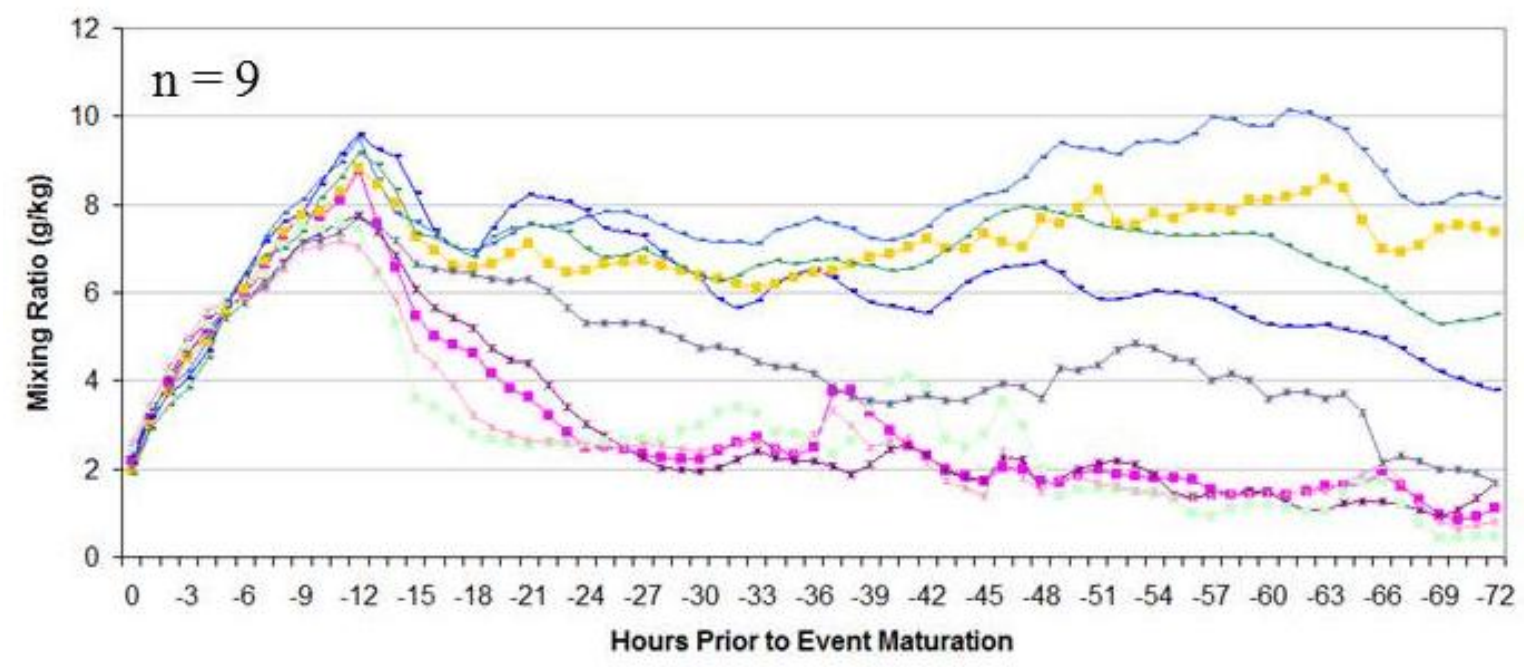

(B) Members $>\mathbf{2}$ km AGL

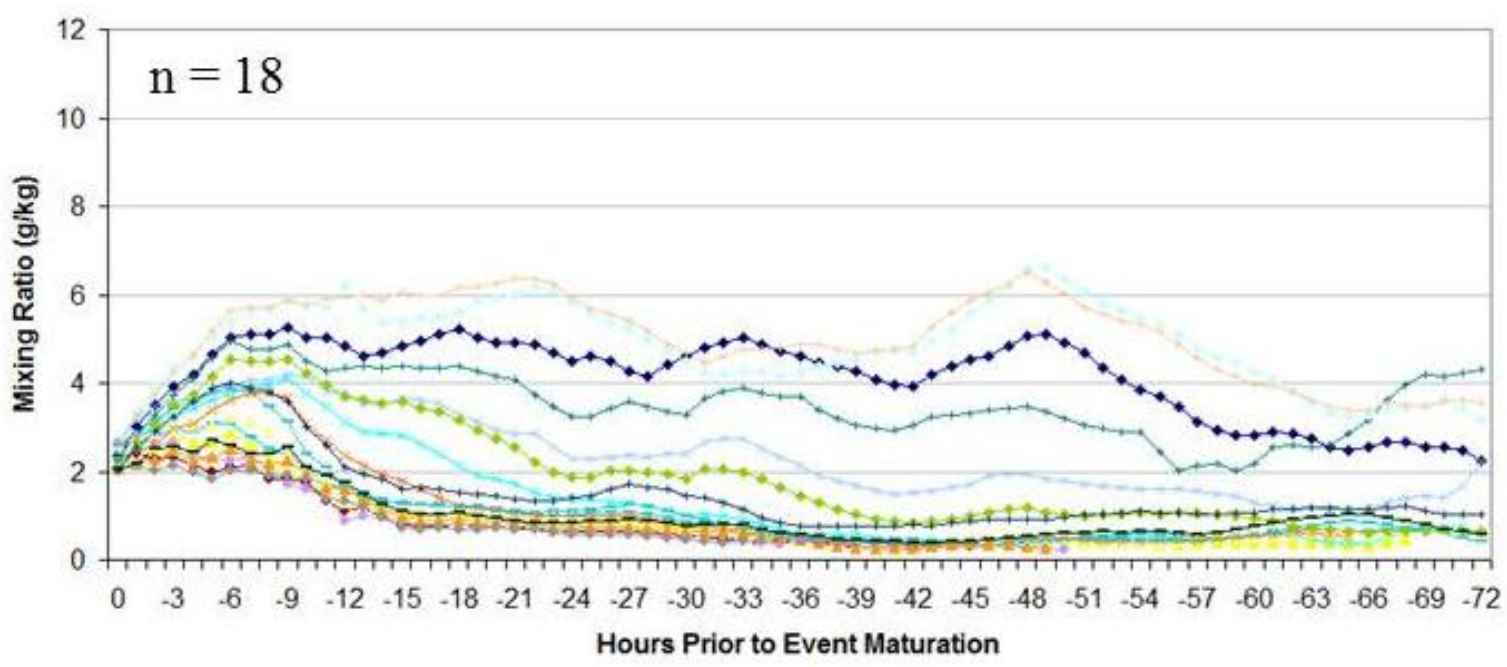

Figure 4.17. Backward ensemble trajectories of moisture content for 06 UTC 5 December 2002 calculated from RDU at the DGZ with altitudes (A) below $2 \mathrm{~km}$ and (B) above $2 \mathrm{~km}$. 


\subsection{2b. Case II: 14 December 2003}

A narrow region of freezing precipitation was observed across several of the western counties in the Raleigh CWA on 14 December 2003. Storm totals ranged from 0.10 in $(0.25$ $\mathrm{cm})$ across the central part of the CWA to $0.50 \mathrm{in}(1.3 \mathrm{~cm})$ in the western part of the CWA (see appendix Figure A13). The distribution of precipitation revealed a narrow transition zone with a region of snow to the north and west and rain across the eastern half of the CWA. In particular, while GSO recorded 0.48 in of freezing rain from this storm, only rain was observed at RDU (Table 4.5). This storm was chosen for further analysis to determine the processes that contributed to variations in precipitation type across the study area.

Examination of back trajectories at GSO and RDU provides insight into the variability in precipitation type across the CWA (Figure 4.18). Of particular importance are the differences in the space-time character and meteorological properties of the near-surface trajectories, as the temperature at this level typically distinguishes between freezing and liquid precipitation. There was little difference in the space-time character and meteorological properties of the WLZ and DGZ trajectories between GSO and RDU. The trend in temperature along the trajectories (Figure 4.19a) revealed that the near-surface air parcels at both GSO and RDU reached a maximum temperature of $4^{\circ} \mathrm{C}$ at $\mathrm{T}-10$ hours and gradually cooled over the remainder of the trajectories. However, the air parcels at GSO continued to cool to a near-surface temperature of $-1.7^{\circ} \mathrm{C}$ while the air parcels at RDU remained just above freezing $\left(0.6^{\circ} \mathrm{C}\right)$.

Examination of $\theta$ values during this time revealed a decrease of $4 \mathrm{~K}$ along the nearsurface GSO trajectory (i.e. diabatic cooling) followed by an increase of $3 \mathrm{~K}$ over the final 3 hours, while $\theta$ increased by $6 \mathrm{~K}$ along the near-surface RDU trajectory (i.e. diabatic 
warming) (Figure 4.19b). Because the near-surface temperatures at GSO and RDU were within just a few degrees of freezing, these relatively small changes in diabatic contributions were significant contributors to the observed differences in precipitation type. The source of diabatic warming of the near-surface RDU trajectory may have been high fluxes of sensible and latent heat from the ocean to the atmosphere, as air parcels traveled closer to the coast during the final 24 hours of the trajectory than those connected with the near-surface GSO trajectory (Figure 4.18).

Figure 4.20 illustrates the space-time character of the near-surface trajectory ensemble members calculated from GSO at the time of heaviest precipitation (06 UTC 14 December 2003). Fourteen of the 27 trajectories originated over the Upper Midwest at an altitude of between 0.4 and $1.8 \mathrm{~km}$ and exhibited below-freezing temperatures at T-0 hours (between -1.0 and $-1.9^{\circ} \mathrm{C}$ ). This group of trajectories closely resembled the composite nearsurface trajectory in Figure 4.1. In contrast, the remaining 13 trajectories originated over south-central Canada and descended nearly $3 \mathrm{~km}$ over the 72 -hr trajectory period. Interestingly, all of these trajectories exhibited above-freezing temperatures at T-0 hours (between 0.2 and $2.1^{\circ} \mathrm{C}$ ). Examination of $\theta$ values revealed a mean increase of $4 \mathrm{~K}$ over the final 12 hours of the trajectories (Figure 4.21a), while $\theta$ values were nearly constant for ensemble members with below-freezing near-surface temperatures (Figure 4.21b). This suggests that the air parcels that warmed to temperatures above freezing encountered a source of diabatic heating over the final 12 hours of the trajectory. The space-time character of the ensemble trajectories (Figure 4.20) suggests that several ensemble members crossed the Atlantic coast during this time, thereby inducing sensible and latent heat fluxes that resulted in a warming of air parcels to above freezing. 
The set of ensemble trajectories terminating in the WLZ is illustrated in Figure 4.22. The general space-time character of these trajectories closely resembled the composite WLZ trajectory in Figure 4.1, except that the ensemble trajectories originated further west and at generally higher altitudes. Similar to the WLZ composite, the ensemble trajectories descended to below $1 \mathrm{~km}$ as they traveled over the Atlantic Ocean for the final 24 hours of the trajectory period. As in the 4-5 December 2002 ice storm (Figure 4.15), the net diabatic contribution to air parcel temperature was calculated for all WLZ ensemble members with comparisons between parcels that were 1 to $4^{\circ} \mathrm{C}$ and 5 to $8^{\circ} \mathrm{C}$. Figure 4.23 reveals that over the 72-hr trajectory period, ensemble members with WLZ temperatures between 1 and $4^{\circ} \mathrm{C}$ exhibited on average as much as $21^{\circ} \mathrm{C}$ of warming due to diabatic processes (rate of $0.3^{\circ} \mathrm{C} / \mathrm{hr}$ ). In contrast, ensemble members with WLZ temperatures between 5 and $8^{\circ} \mathrm{C}$ exhibited $25^{\circ} \mathrm{C}$ of warming due to diabatic processes (rate of $0.4^{\circ} \mathrm{C} / \mathrm{hr}$ ). This further supports the notion that warmer WLZ temperatures are associated with greater rates of diabatic warming over the course of the trajectory period. Figure 4.24 illustrates the set of ensemble trajectories terminating at the DGZ. Fourteen of the 27 ensemble members generally resembled the composite DGZ-Pacific trajectory and remained above $3.5 \mathrm{~km}$ over the entire trajectory period. In contrast, the remaining 13 ensemble members more closely resembled the DGZ-Gulf trajectory and remained below $2 \mathrm{~km}$ for most of the trajectory period. As in the 4-5 December 2002 ice storm (Figure 4.17), maximum moisture contents for ensemble members below $2 \mathrm{~km}$ were greater than the moisture contents observed for ensemble members above $2 \mathrm{~km}(6.5 \mathrm{~g} / \mathrm{kg}$ compared to $4.0 \mathrm{~g} / \mathrm{kg}$; Figure 4.25$)$. Ensemble members with higher moisture contents exhibited well-defined evaporation-precipitation cycles and greater rates of condensation and deposition over the final 12 hours of the trajectories. 
Table 4.5. Hourly precipitation totals (in. liquid equivalent) and present weather for the 14 December 2003 ice storm at RDU and GSO. Back trajectories were computed from the time highlighted in boldface.

\begin{tabular}{|c|c|c|c|c|c|}
\hline \multirow{2}{*}{ Date } & \multirow{2}{*}{$\begin{array}{c}\text { Time } \\
\text { (UTC) }\end{array}$} & \multicolumn{2}{|c|}{ RDU } & \multicolumn{2}{|c|}{ GSO } \\
\hline & & $\begin{array}{c}\text { Precip } \\
\text { (Liq. Equiv.) }\end{array}$ & $\begin{array}{l}\text { Present } \\
\text { Weather }\end{array}$ & $\begin{array}{c}\text { Precip } \\
\text { (Liq. Equiv.) }\end{array}$ & $\begin{array}{l}\text { Present } \\
\text { Weather }\end{array}$ \\
\hline $12 / 14 / 2003$ & 01 & $\operatorname{Tr}$ & -RA & 0.01 & -FZRA \\
\hline $12 / 14 / 2003$ & 02 & $\operatorname{Tr}$ & RA & 0.03 & -FZRA BR \\
\hline $12 / 14 / 2003$ & 03 & 0.04 & RA & 0.05 & FZRA BR \\
\hline $12 / 14 / 2003$ & 04 & 0.04 & RA BR & 0.08 & FZRA BR \\
\hline $12 / 14 / 2003$ & 05 & 0.03 & RA BR & 0.10 & FZRA \\
\hline $12 / 14 / 2003$ & 06 & 0.13 & RA BR & 0.08 & FZRA \\
\hline $12 / 14 / 2003$ & 07 & 0.25 & RA BR & & -FZRA BR \\
\hline $12 / 14 / 2003$ & 08 & 0.10 & RA BR & 0.03 & -FZRA \\
\hline $12 / 14 / 2003$ & 09 & 0.06 & -RA & 0.06 & -FZRA \\
\hline $12 / 14 / 2003$ & 10 & 0.11 & RA BR & 0.03 & -FZRA \\
\hline $12 / 14 / 2003$ & 11 & 0.09 & -RA BR & 0.01 & -FZRA \\
\hline $12 / 14 / 2003$ & 12 & 0.01 & -RA & $\operatorname{Tr}$ & \\
\hline $12 / 14 / 2003$ & 13 & 0.01 & -RA & $\operatorname{Tr}$ & $-\mathrm{RA}$ \\
\hline $12 / 14 / 2003$ & 14 & 0.01 & -RA & $\operatorname{Tr}$ & $\mathrm{BR}$ \\
\hline $12 / 14 / 2003$ & 15 & 0.01 & & $\operatorname{Tr}$ & -FZDZ BR \\
\hline $12 / 14 / 2003$ & 16 & $\operatorname{Tr}$ & -RA & $\operatorname{Tr}$ & -DZ BR \\
\hline $12 / 14 / 2003$ & 17 & 0.01 & BR & $\operatorname{Tr}$ & -DZ BR \\
\hline $12 / 14 / 2003$ & 18 & 0.01 & RA FG & 0.02 & -DZ BR \\
\hline $12 / 14 / 2003$ & 19 & 0.05 & -RA BR & 0.02 & -DZ BR \\
\hline $12 / 14 / 2003$ & 20 & $\operatorname{Tr}$ & & $\operatorname{Tr}$ & \\
\hline $12 / 14 / 2003$ & 21 & 0.01 & -RA BR & & \\
\hline $12 / 14 / 2003$ & 22 & $\operatorname{Tr}$ & -RA BR & & \\
\hline
\end{tabular}

$* \mathrm{RA}=$ rain $; \mathrm{BR}=$ mist $\mathrm{DZ}=$ drizzle $; \mathrm{SN}=$ snow $; \mathrm{FZRA}=$ freezing rain; $\mathrm{FZDZ}=$ freezing drizzle 

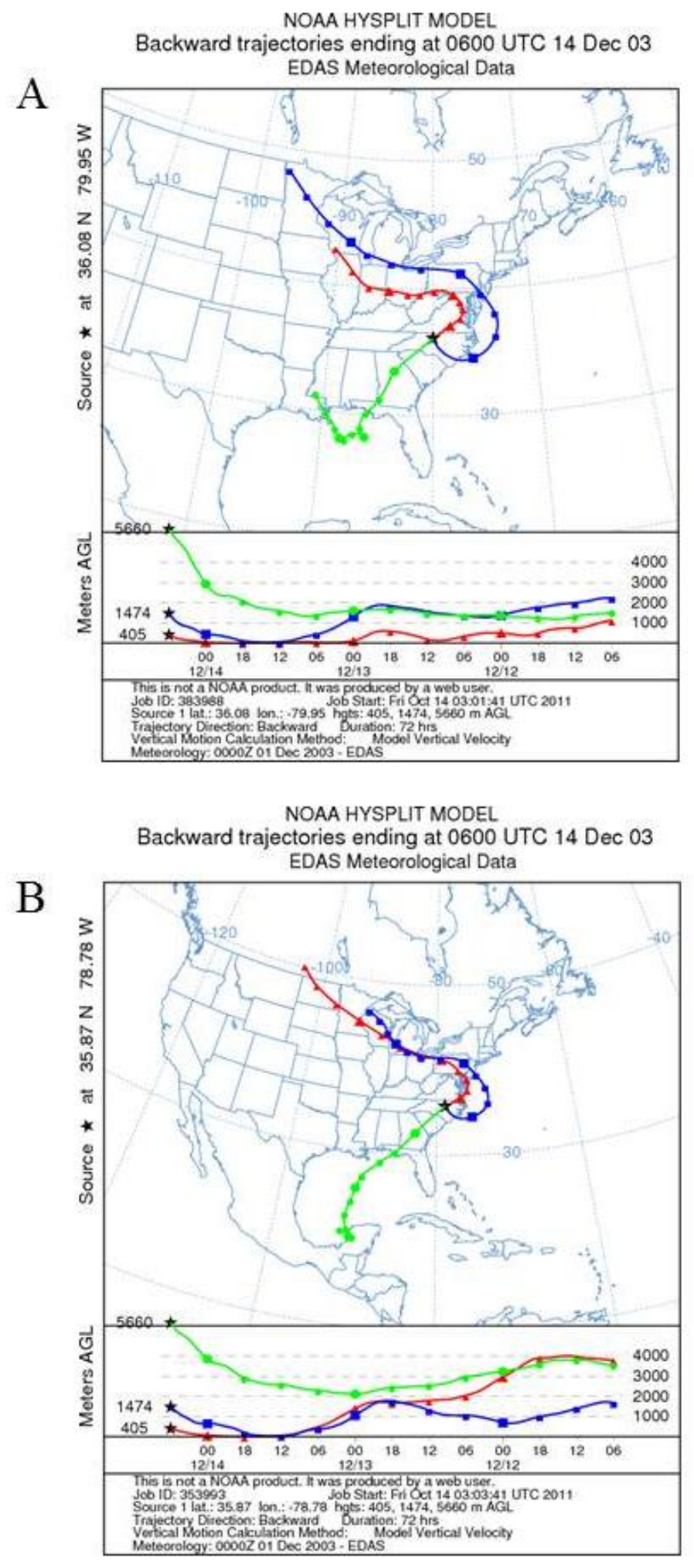

Figure 4.18. Horizontal and vertical trajectories for 06 UTC 14 December 2003 terminating at the near-surface level (red trajectories), WLZ (blue trajectories), and DGZ (green trajectories) at (A) GSO and (B) RDU. 

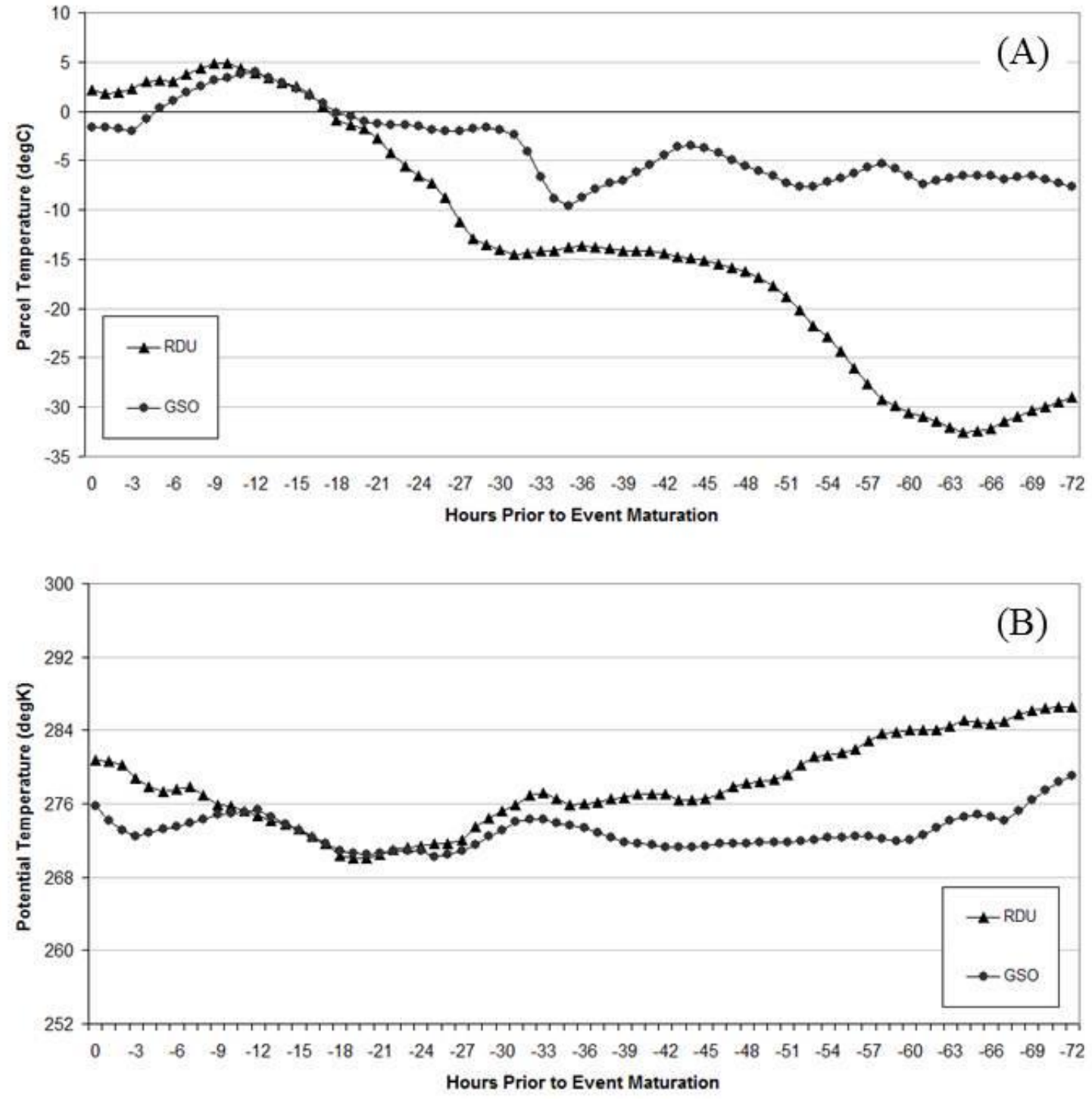

Figure 4.19. Air parcel trajectories of (A) temperature and (B) potential temperature for 06 UTC 14 December 2003 terminating at the near-surface layer at RDU and GSO. 


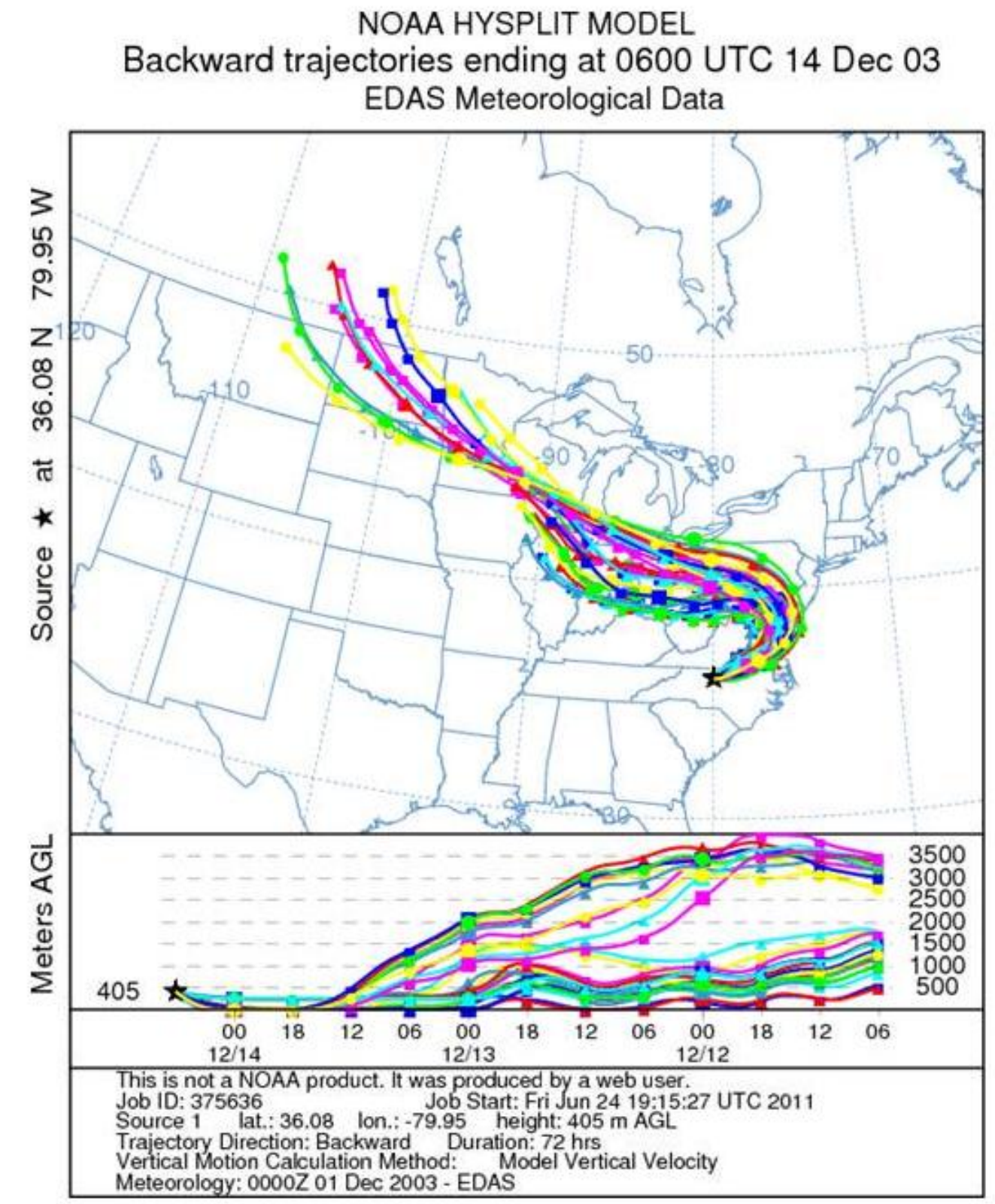

Figure 4.20. Backward ensemble trajectories for 06 UTC 14 December 2003 calculated from GSO at the near-surface level. Vertical trajectories are shown in the bottom panel. 


\section{(A) Members with $975 \mathrm{hPa} \mathbf{T}>\mathbf{0}$ degC}

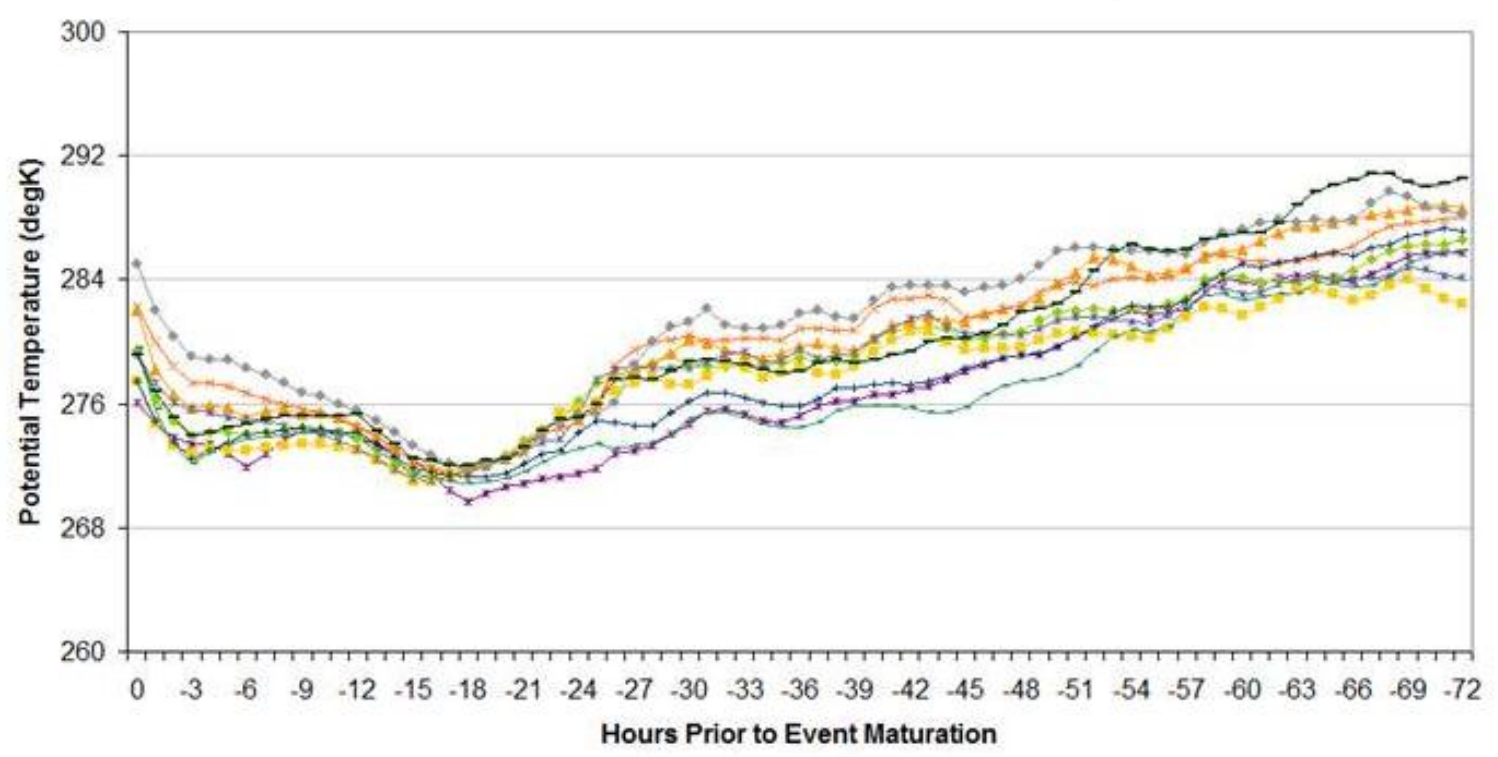

(B) Members with $975 \mathrm{hPa} \mathbf{T}<\mathbf{0} \operatorname{degC}$

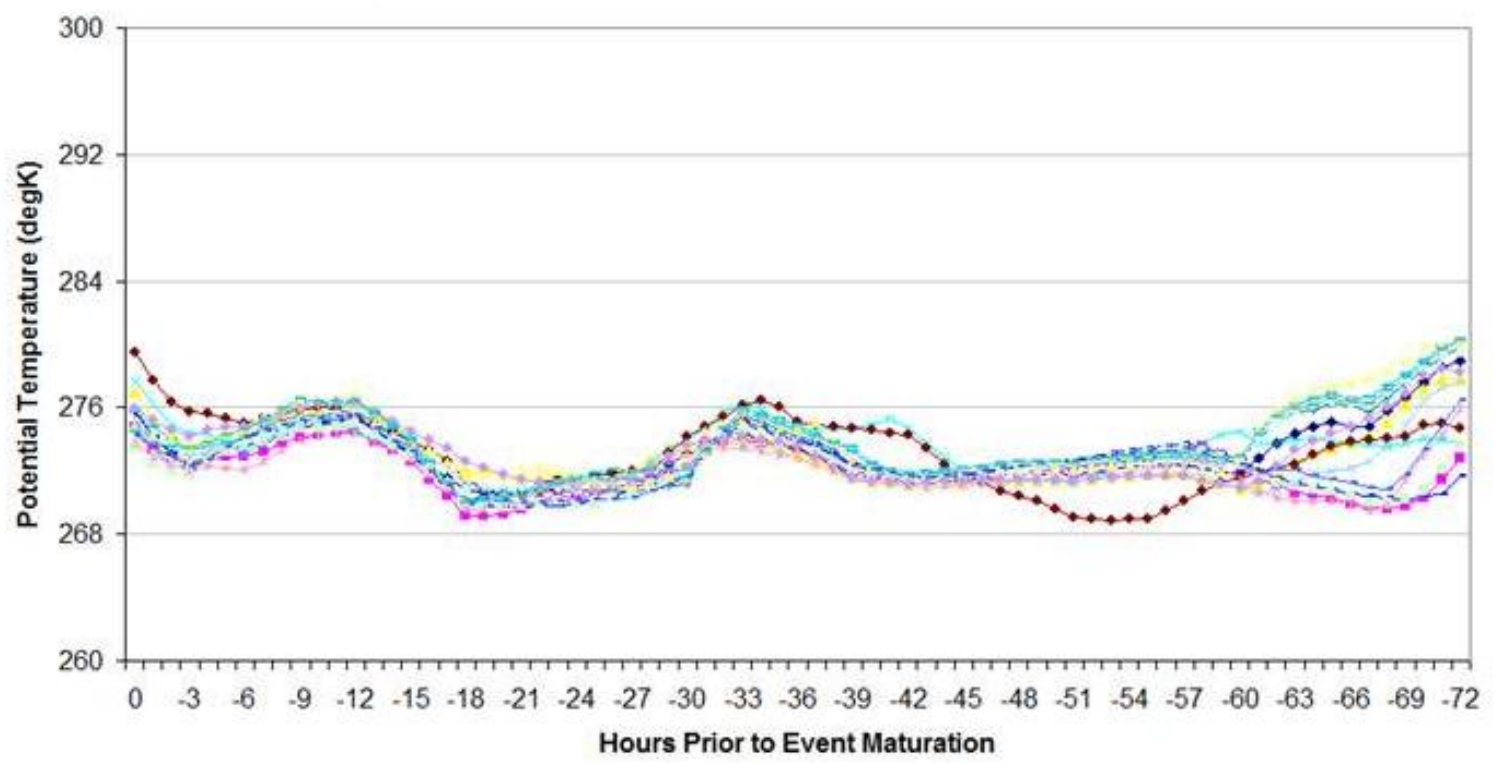

Figure 4.21. Backward ensemble trajectories of moisture content for 06 UTC 14 December 2002 calculated from RDU with near-surface temperatures (A) above freezing and (B) below freezing. 


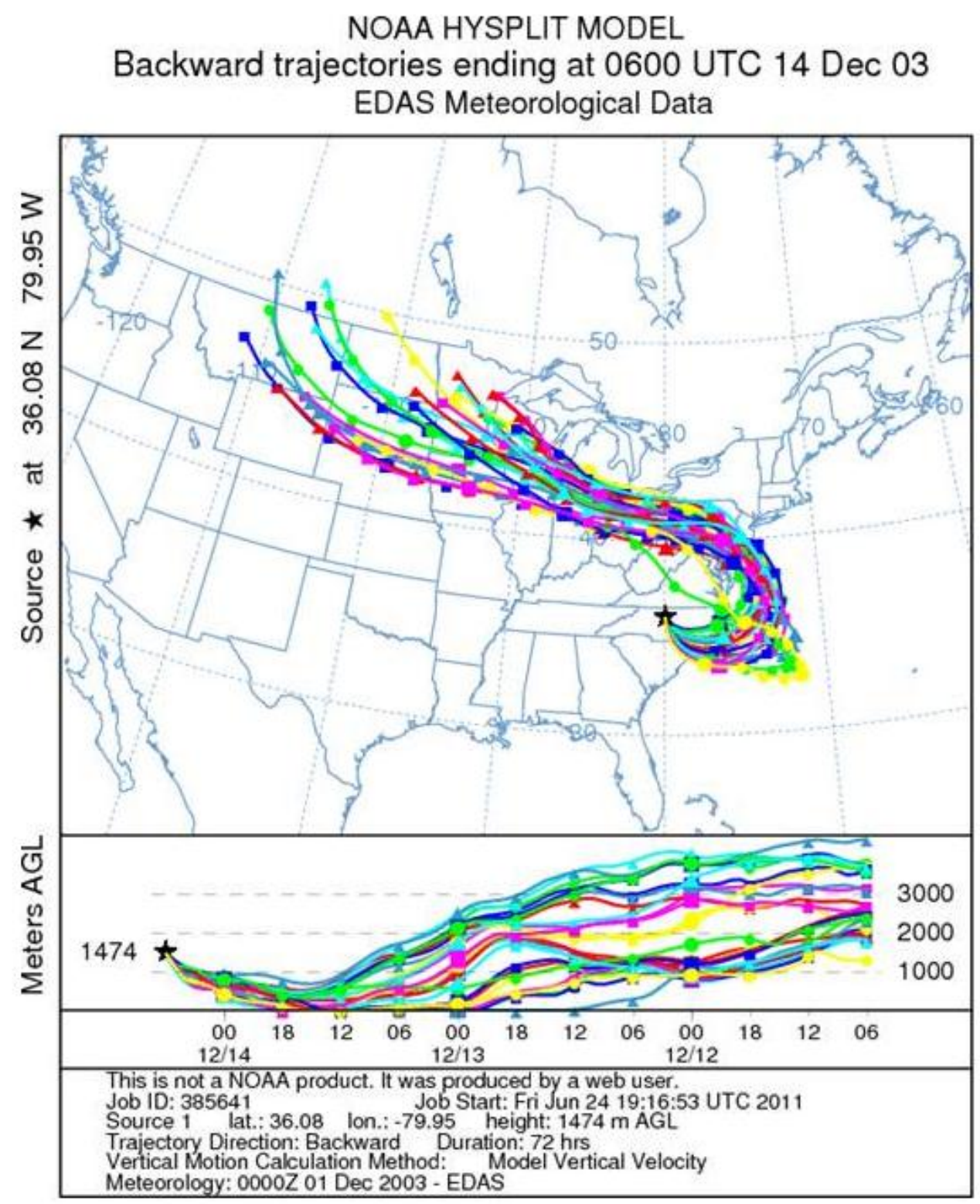

Figure 4.22. Same as Figure 4.20, except at the WLZ. 


\section{(A) Members with $850 \mathrm{hPa}$ T 5-8 degC}

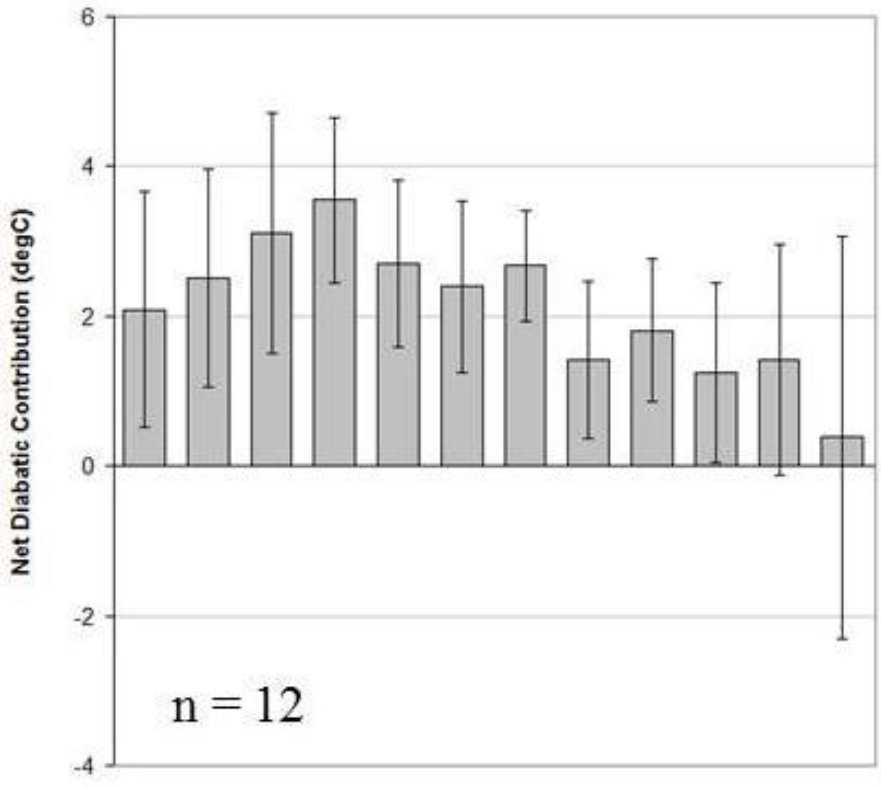

(B) Members with $850 \mathrm{hPa}$ T 1-4 degC

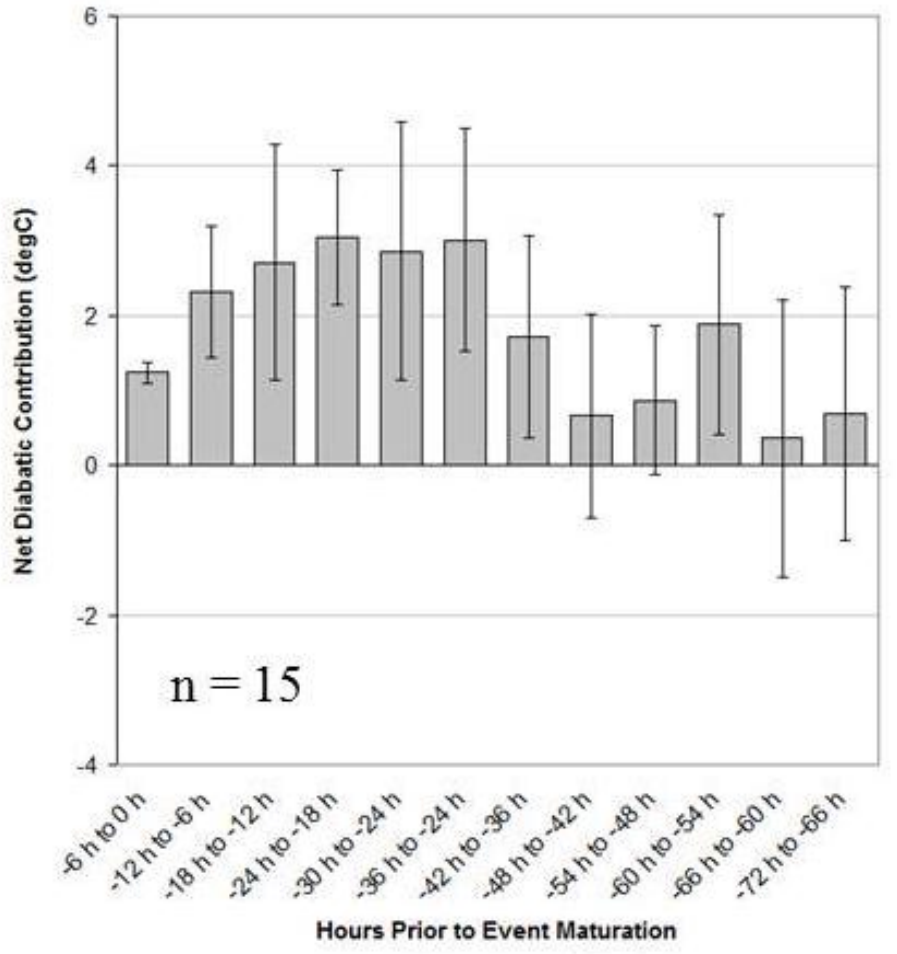

Figure 4.23. Net diabatic contribution to air parcel temperature trajectories at RDU with WLZ temperatures (A) between 5 and $8^{\circ} \mathrm{C}$ and (B) between 1 and $4^{\circ} \mathrm{C}$ at 6 -hr trajectory segments. Range bars indicate the maximum and minimum values among the ensemble members. 


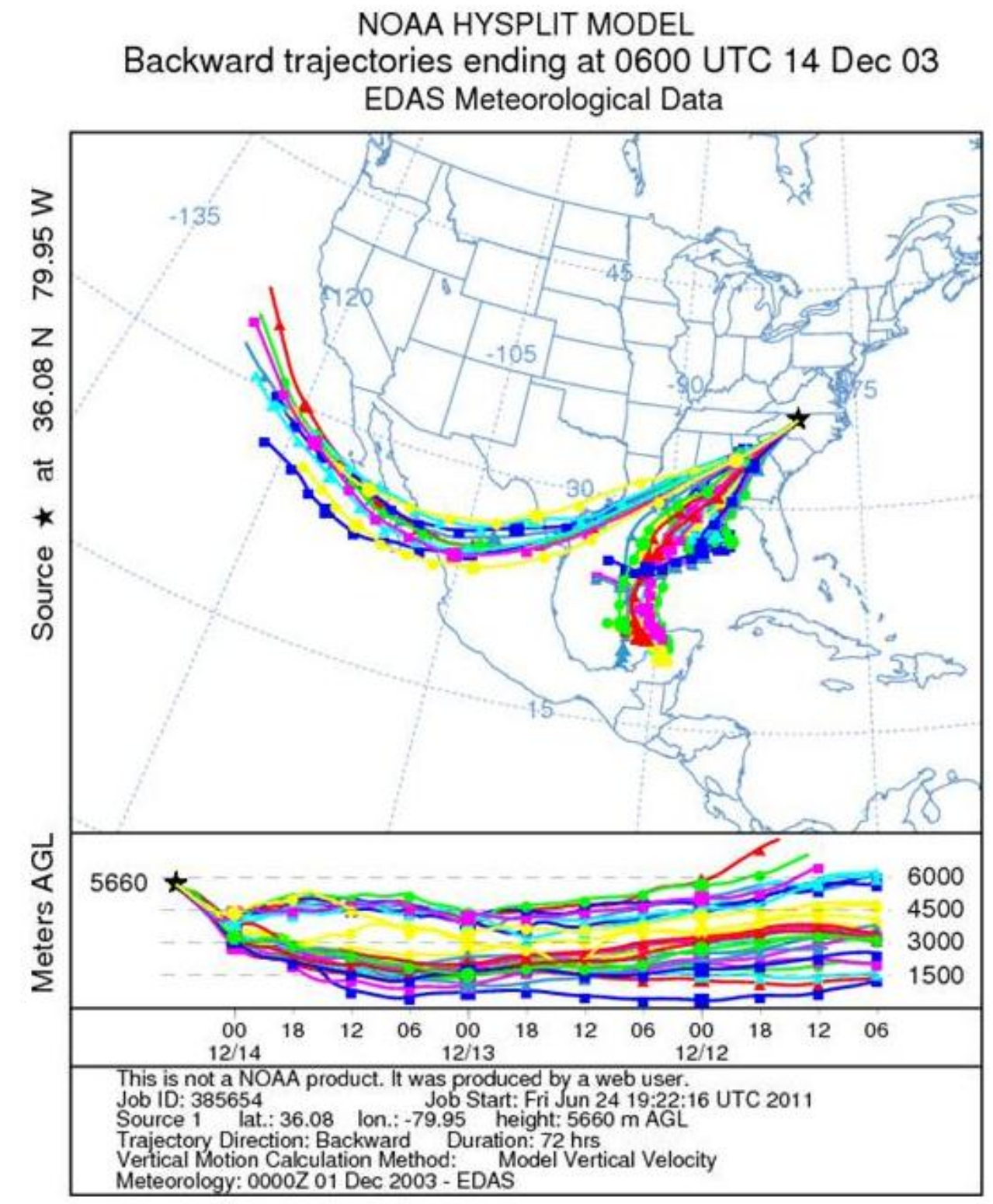

Figure 4.24. Same as Figure 4.20, except at the DGZ. 
(A) Members $<\mathbf{2} \mathbf{~ k m ~ A G L ~}$

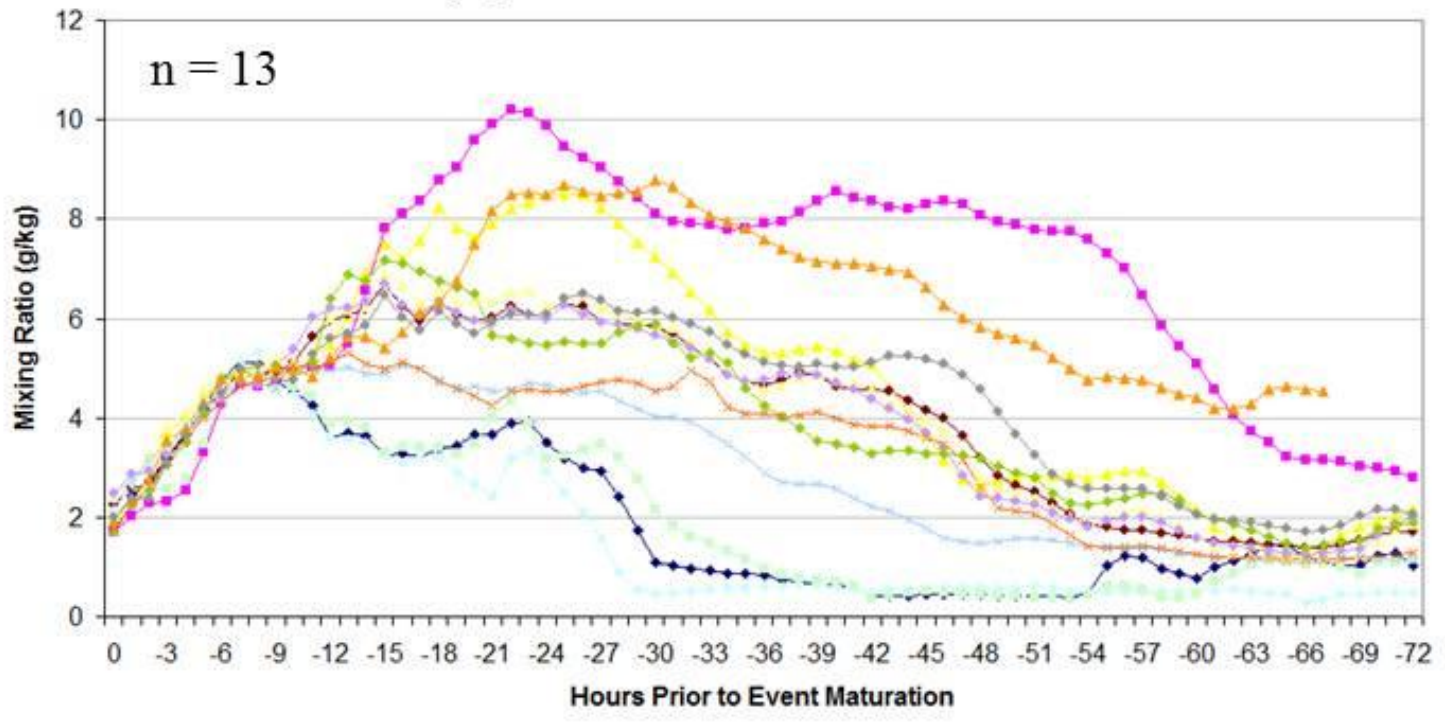

(B) Members $>\mathbf{2} \mathbf{~ k m ~ A G L ~}$

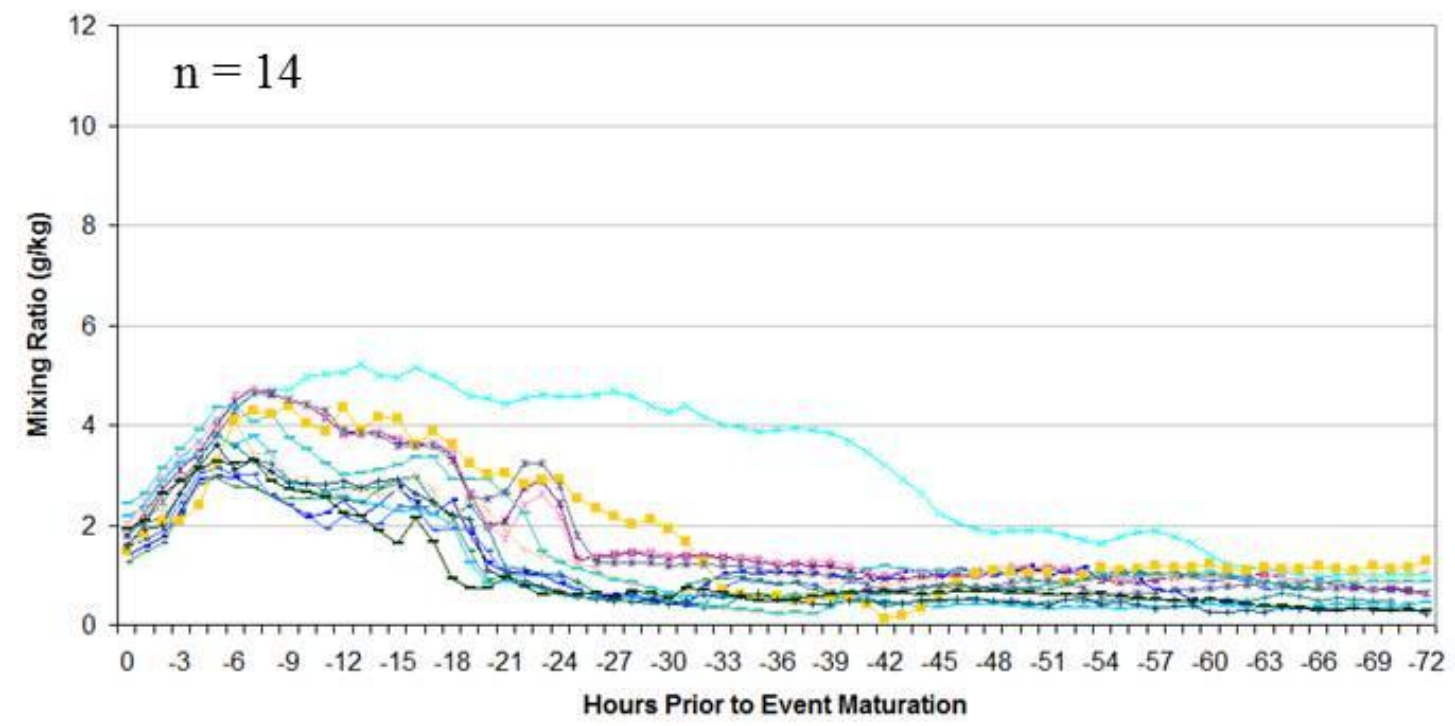

Figure 4.25. Backward ensemble moisture trajectories for 06 UTC 14 December 2003 calculated from GSO at the DGZ with altitudes (A) below $2 \mathrm{~km}$ and (B) above $2 \mathrm{~km}$. 


\subsection{Synoptic Analysis}

As in Chapter 3, the synoptic-scale circulation responsible for the back trajectory motions in the heavy ice storms was explored using composites of low-level geopotential height fields (i.e. 1000 and $850 \mathrm{hPa}$ ). Because of the greater altitude exhibited by the DGZ trajectories (see Figure 4.2) composites of $500 \mathrm{hPa}$ geopotential heights were also examined. Comparisons were made between ice storms characterized by DGZ-Gulf and DGZ-Pacific trajectories.

\subsubsection{Near-Surface Trajectory}

As previously illustrated, the near-surface trajectory exhibited similar space-time characteristics to its snowstorm counterpart, with anticyclonic curvature and gradual descent over the 72-hr trajectory period. The near-surface ice storm trajectory also originated along the eastern periphery of a surface anticyclone, except in this case the anticyclone continued to build eastward over the Great Lakes between T-48 and T-24 hours (Figure 4.26). Cold-air damming (CAD) was evident in the $1000 \mathrm{hPa}$ composite at $\mathrm{T}-24$ hours, as indicated by an inverted geopotential height ridge in the lee of Appalachian Mountains. This resulted in northeasterly flow near the surface, as indicated by the pronounced anticyclonic curvature of the near-surface trajectory over the last 24 hours of the trajectory period. By T-0 hours the anticyclone was located over the Northeast U.S. with a weak area of low pressure across the Southeast region. The composite map revealed two inverted geopotential height troughs, one located over Tennessee and Kentucky (associated with the primary cyclone) and a more subtle one located along the Georgia and South Carolina coast (associated with the secondary cyclone). The CAD signature was less evident in the composite at T-0 hours due to the 
weakening of the anticyclone, resulting in a decrease in cold-air advection into central North Carolina. However, because the source region of the near-surface trajectory was characterized by an exceptionally cold air mass (Figure 4.3), the near-surface air parcel temperature was able to maintain a temperature supportive of freezing precipitation by the end of the trajectory.

\subsubsection{Warm Layer Zone Trajectory}

As in the snowstorm composite, the $850 \mathrm{hPa}$ circulation exhibited northwesterly flow across the Great Lakes region from T-72 to T-48 hours, which corresponded well with the WLZ trajectory (Figure 4.27). However, in this case an $850 \mathrm{hPa}$ ridge was located across the southern U.S. with a deepening trough located across the Great Plains. Between T-48 and T24 hours the ridge over the southern U.S. shifted to the east while the trough amplified into a closed low over the Midwest. This coincided with an increase in anticyclonic curvature along the WLZ trajectory as it traveled over the Atlantic Ocean off the North Carolina coast. As the $850 \mathrm{hPa}$ trough deepened into a closed low between T-24 and T-0 hours, the WLZ trajectory was entrained into a low-level circulation and ascended approximately $1 \mathrm{~km}$ as it entered the WLZ from the southeast. This is in contrast to the snowstorm composite, which revealed a strong low off the Southeast coast and a cyclonically-curving WLZ trajectory with little vertical motion. In that case, an abrupt shift in the WLZ trajectory coincided with rapidly-evolving synoptic-scale circulation features, including the development and intensification of a surface cyclone off the Southeast coast. 


\subsubsection{Dendritic Growth Zone Trajectories}

Variations in the 1000, 850, and $500 \mathrm{hPa}$ geopotential height fields between ice storms with DGZ-Pacific and DGZ-Gulf trajectories were examined at T-24 and T-0 hours. In addition to their source region, these DGZ trajectories were distinguished by their altitudes; DGZ-Gulf trajectories resided below $2 \mathrm{~km}$ most of the time, while DGZ-Pacific trajectories remained above $3 \mathrm{~km}$. As a result, DGZ-Gulf trajectories exhibited much higher moisture contents and greater lift compared to DGZ-Pacific trajectories. The evolving synoptic-scale circulation associated with DGZ-Pacific trajectories revealed a quasistationary anticyclone over the Northeast U.S. with an area of low pressure located across the Southeast U.S. (Figure 4.28). The corresponding 850 and $500 \mathrm{hPa}$ composites indicated weak shortwave troughs moving east across the Great Plains and Midwest from T-24 to T-0 hours.

In contrast, ice storms with a DGZ-Gulf trajectory exhibited a weakening of the surface anticyclone over the Northeast U.S. but a stronger area of low pressure located across the Southeast region (Figure 4.29). In this case, the $1000 \mathrm{hPa}$ composite revealed an inverted geopotential height trough over the Southeast coast, indicative of Miller-B type cyclogenesis. Indeed, a manual examination of surface weather maps for ice storms associated with DGZGulf trajectories (not shown) revealed secondary cyclogenesis off of the Southeast coast in all four cases. Additionally, the $850 \mathrm{hPa}$ composite indicated a closed low over the Great Plains at T-24 hours, which propagated eastward into the Midwest by T-0 hours (Figure 4.29). At $500 \mathrm{hPa}$, a pronounced shortwave trough over the Great Plains deepened as it moved into the Midwest between T-24 and T-0 hours. This suggests that ice storms with a DGZ-Gulf trajectory are associated with stronger low and mid-level southwesterly flow 
compared to storms with a DGZ-Pacific trajectory. This stronger southwesterly flow advected warm, moist air within the marine atmospheric boundary layer over the Gulf of Mexico, and this moisture contributed to higher maximum precipitation rates over central North Carolina. 


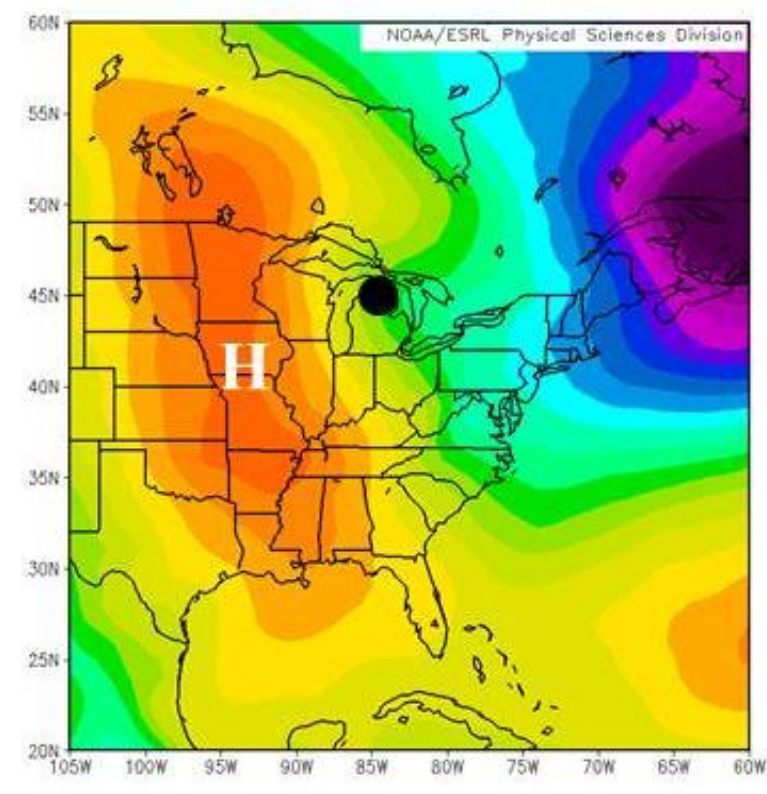

T-72

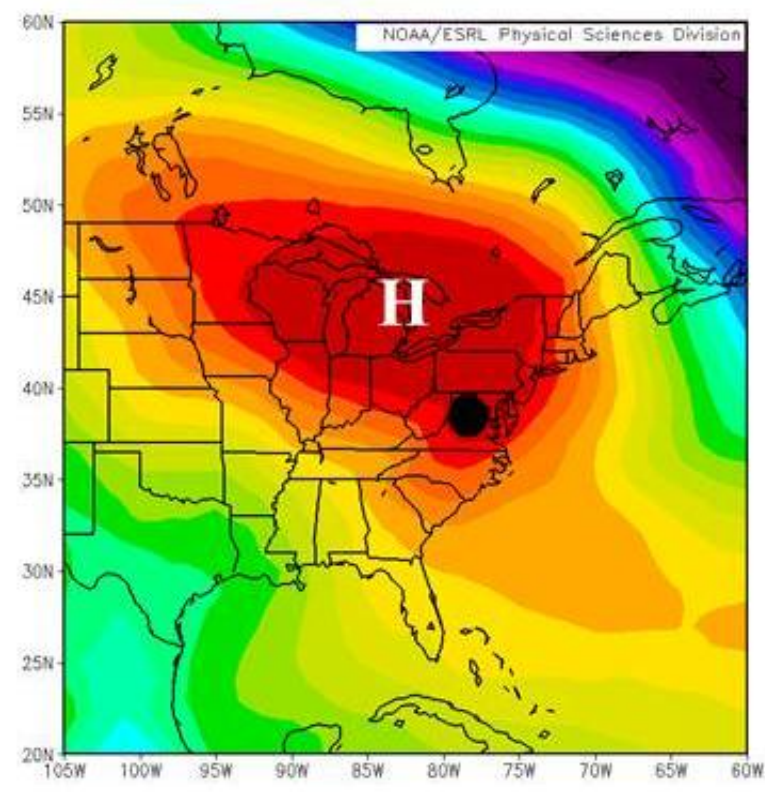

T-24

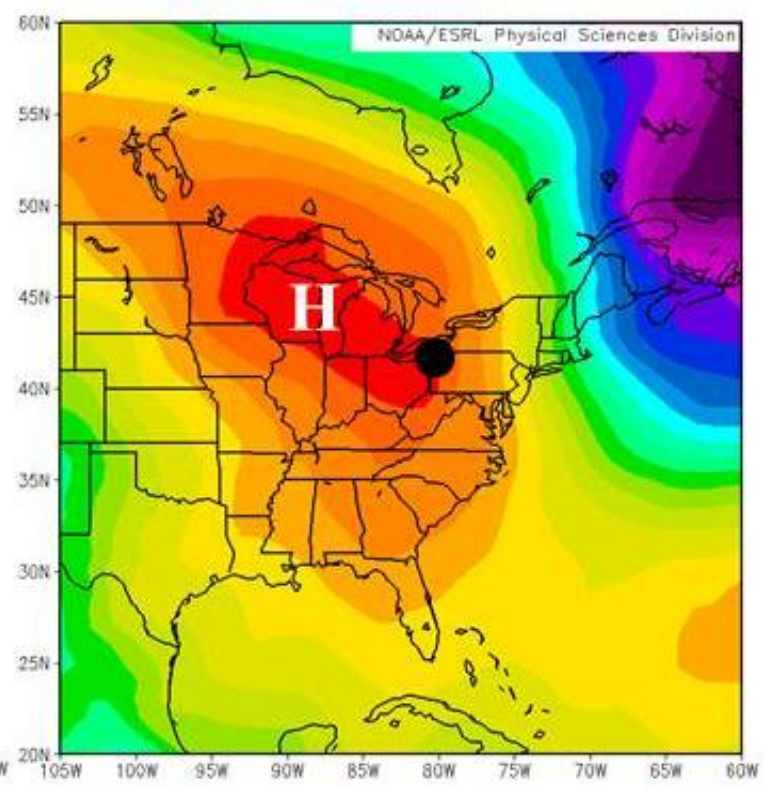

T-48
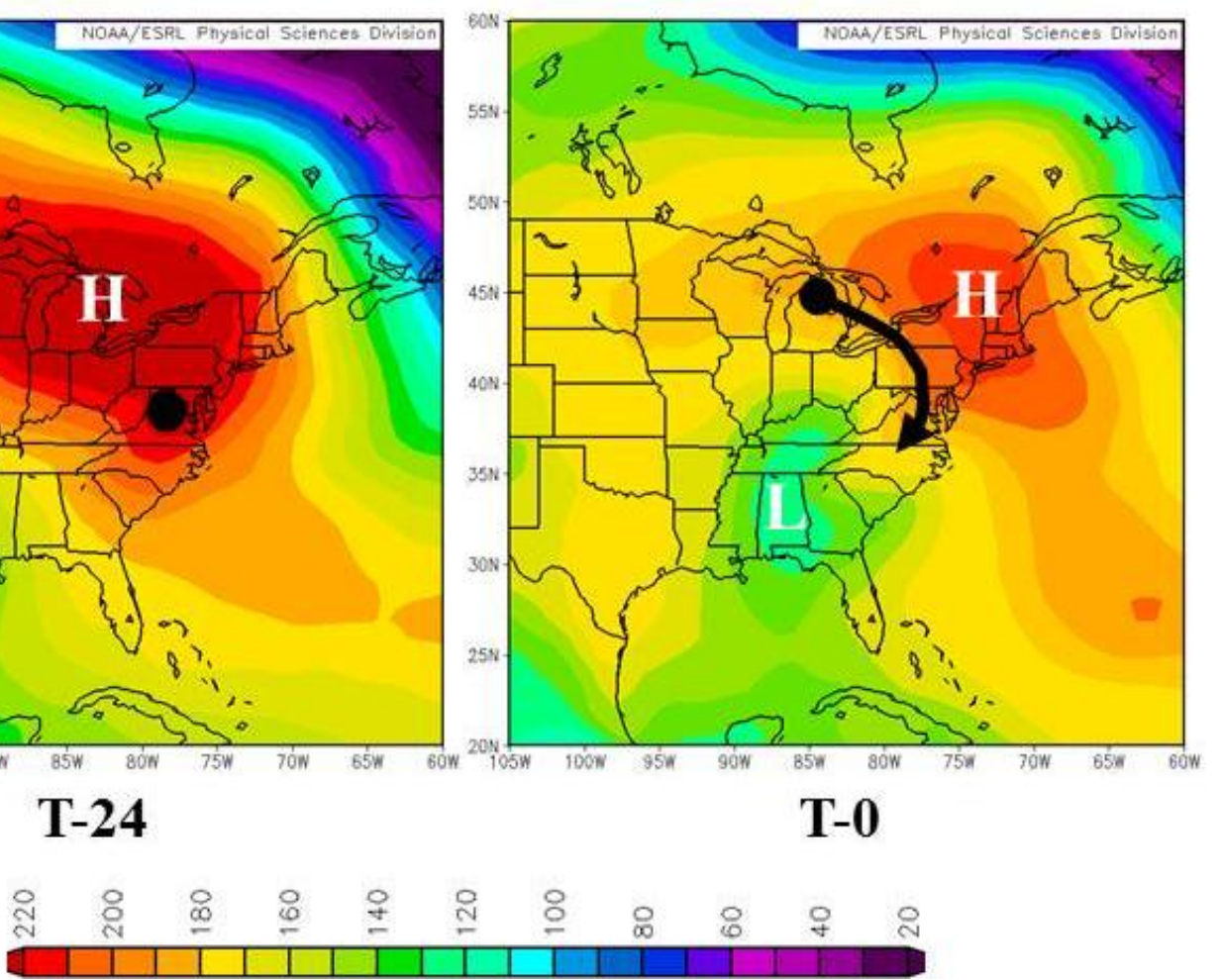

Figure 4.26. Composite analysis of $1000 \mathrm{hPa}$ geopotential heights (meters) for all seven ice storms at 24-hr intervals from T-72 to T-0 hours. Areas of high pressure (" $\mathrm{H}$ ") and low pressure ("L") are noted. The position of the air parcel at each time step is denoted by a black circle. A sketch of the composite near-surface trajectory is denoted with a solid black line and arrow. 


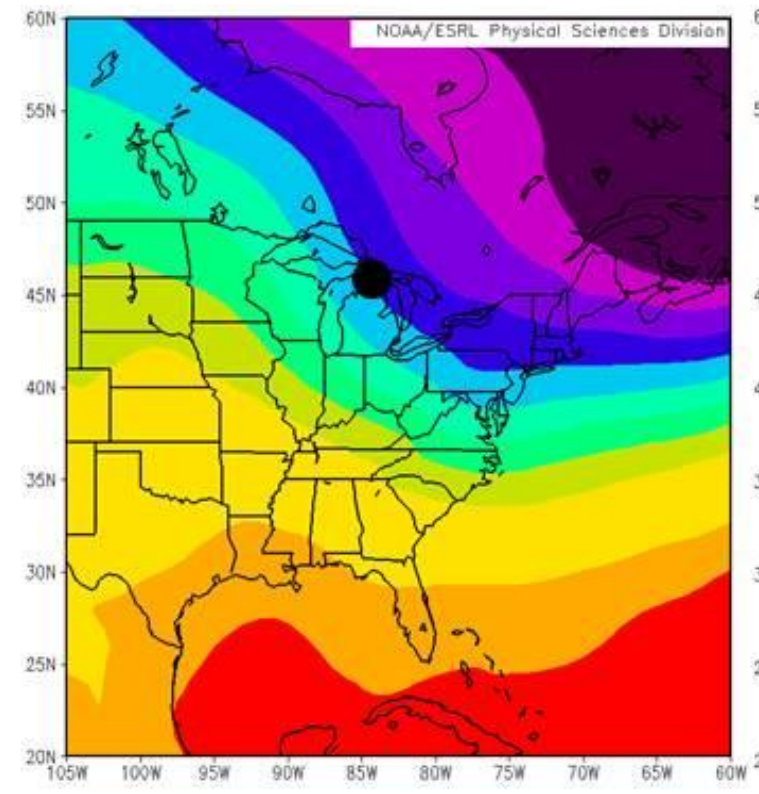

T-72

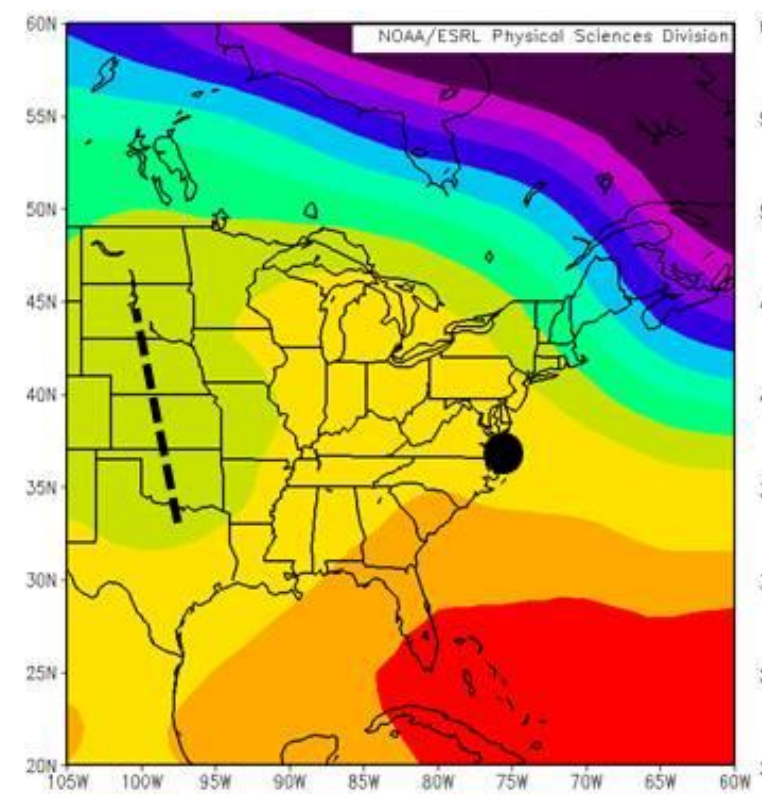

T-24

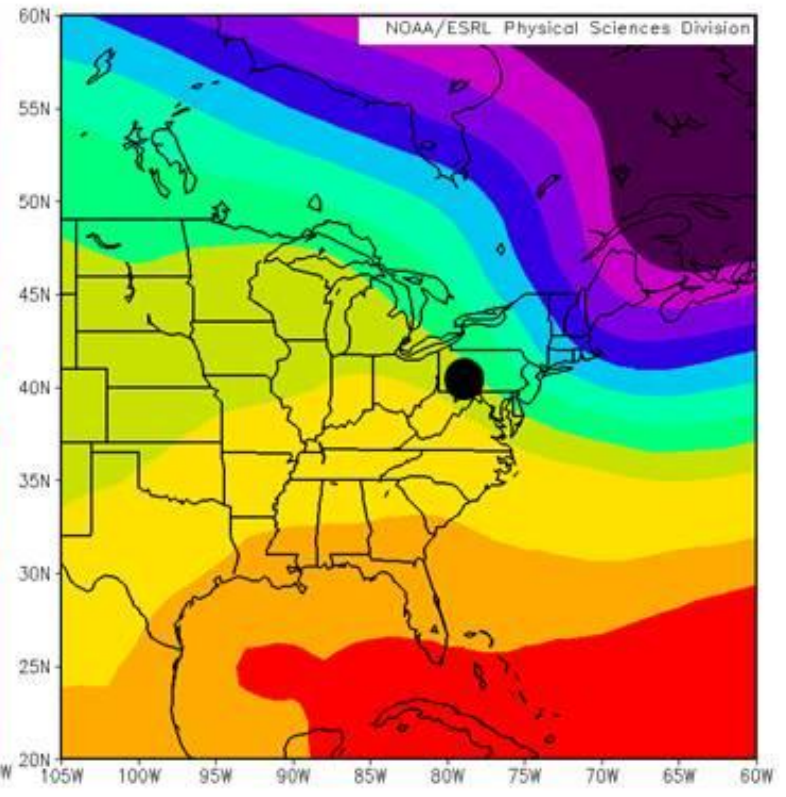

T-48

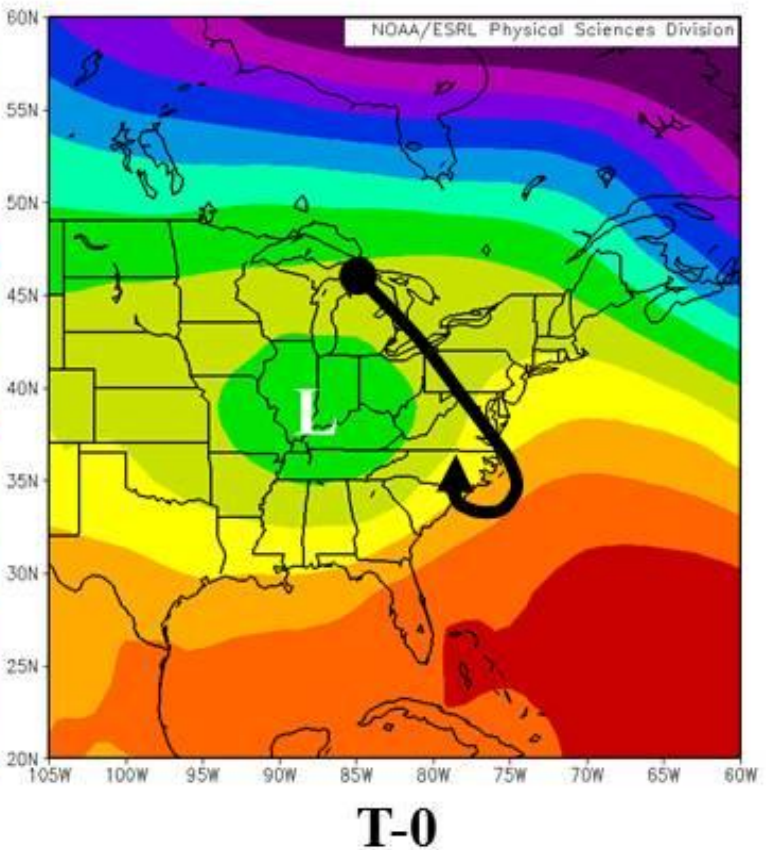

T-0

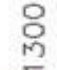

Figure 4.27. Same as Figure 3.27, except of $850 \mathrm{hPa}$ geopotential heights (meters). The position of the air parcel at each time step is denoted by a black circle. A sketch of the composite WLZ trajectory is denoted with a solid black line and arrow. The $850 \mathrm{hPa}$ trough axis at T-24 hours is denoted with a black dashed line. A composite closed low ("L") is noted at T-0 hours. 

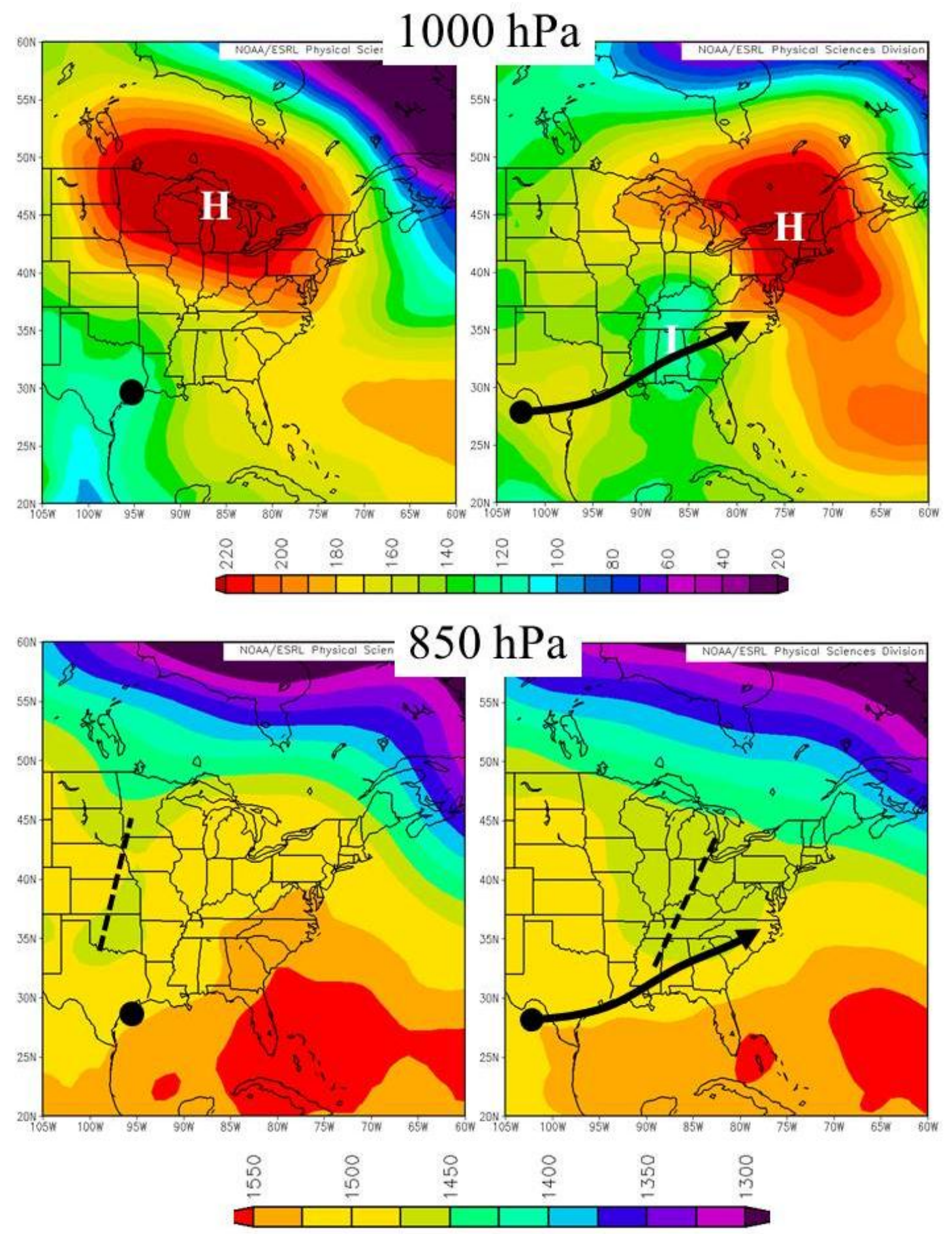

Figure 4.28. Composite analysis of 1000,850 , and $500 \mathrm{hPa}$ geopotential heights (meters) for ice storms with DGZ-Pacific trajectories at T-24 hours (left column) and T-0 hours (right column). The position of the air parcel at each time step is denoted by a black circle. A sketch of the composite DGZ-Pacfic trajectory is denoted with a solid black line and arrow. The $850 \mathrm{hPa}$ trough axis is denoted by the black dashed line. 


\section{$500 \mathrm{hPa}$}
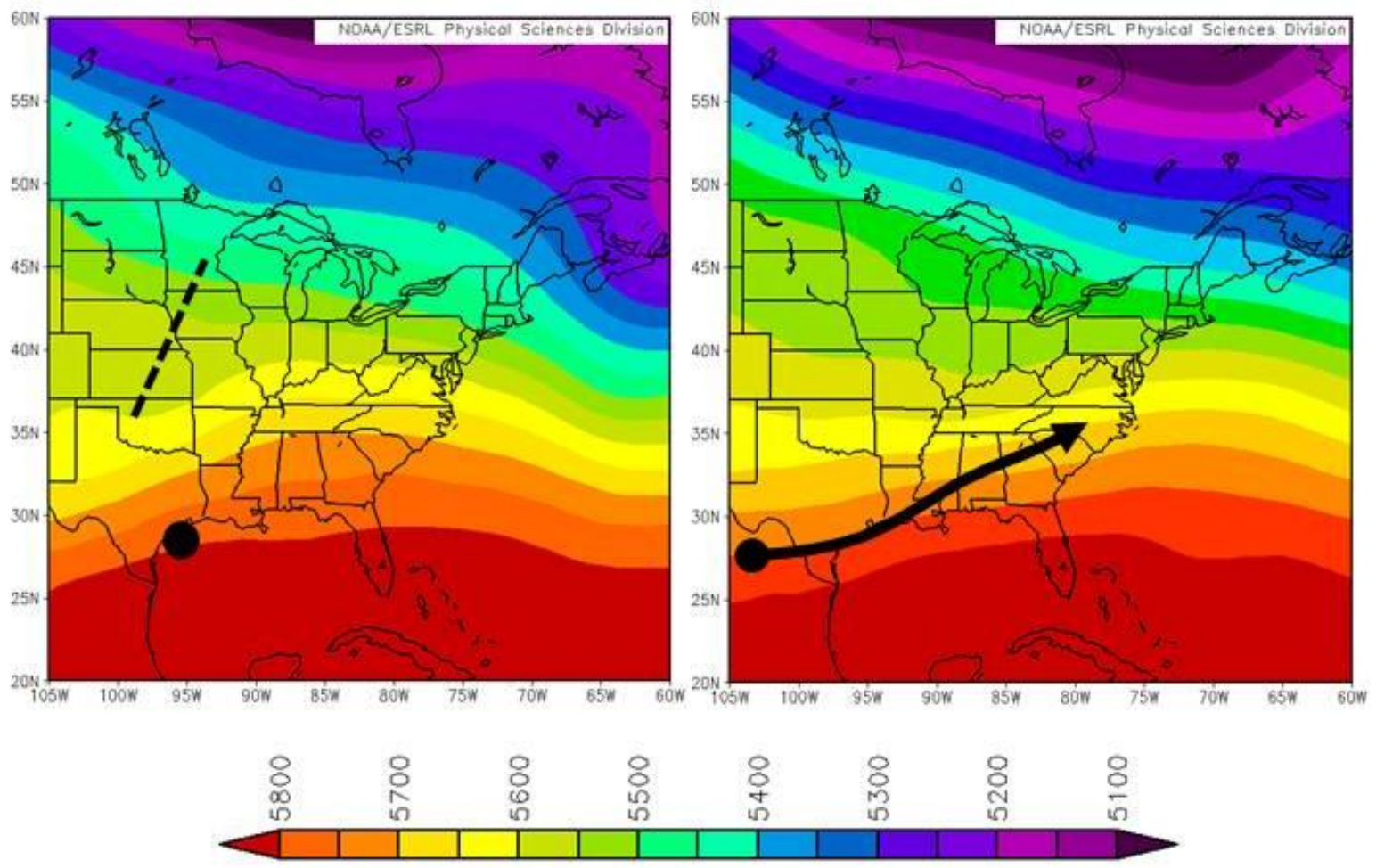

Figure 4.28. Continued 

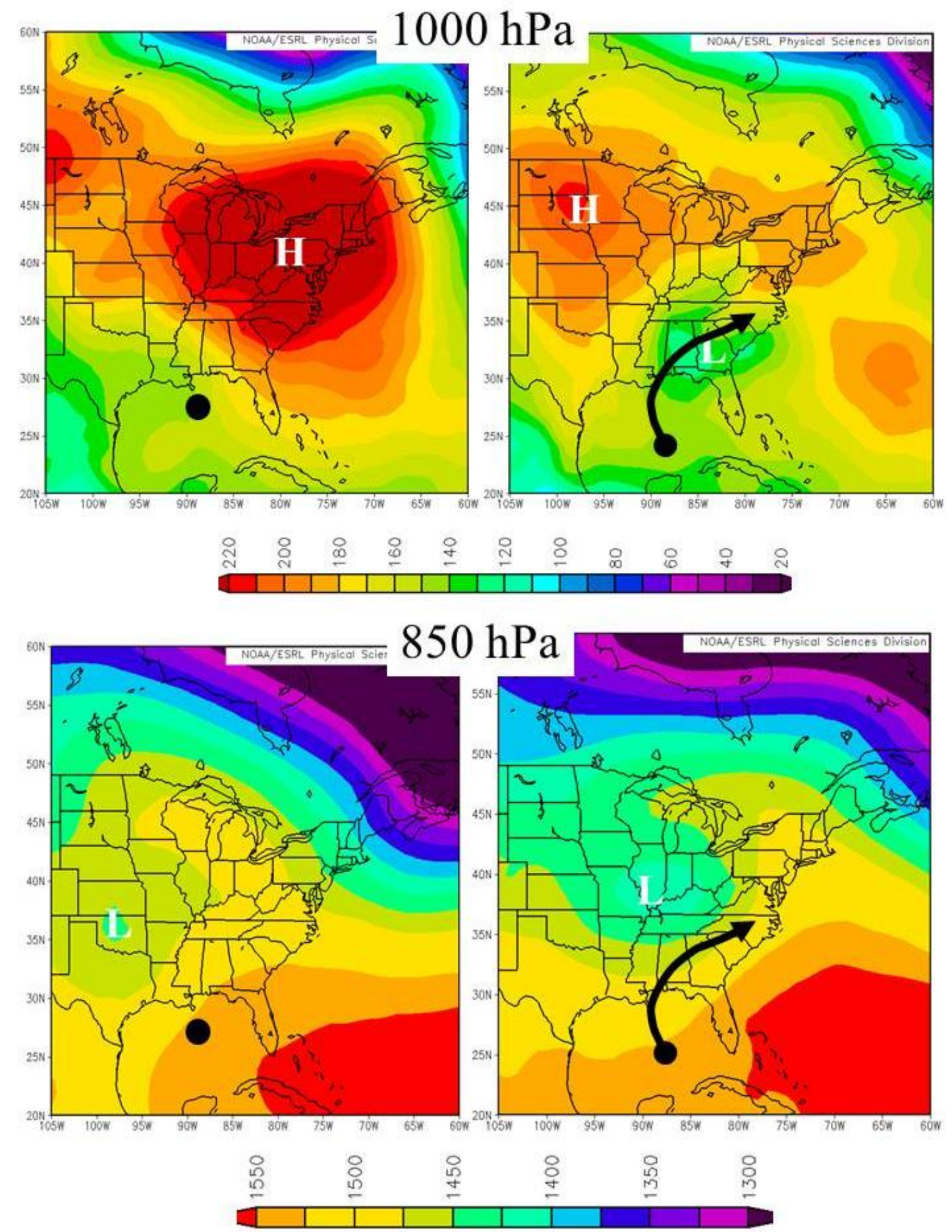

Figure 4.29. Same as Figure 4.28, except for ice storms with DGZ-Gulf trajectories. The position of the air parcel at each time step is denoted by a black circle. A sketch of the composite DGZ-Gulf trajectory is denoted with a solid black line and arrow. 


\section{$500 \mathrm{hPa}$}
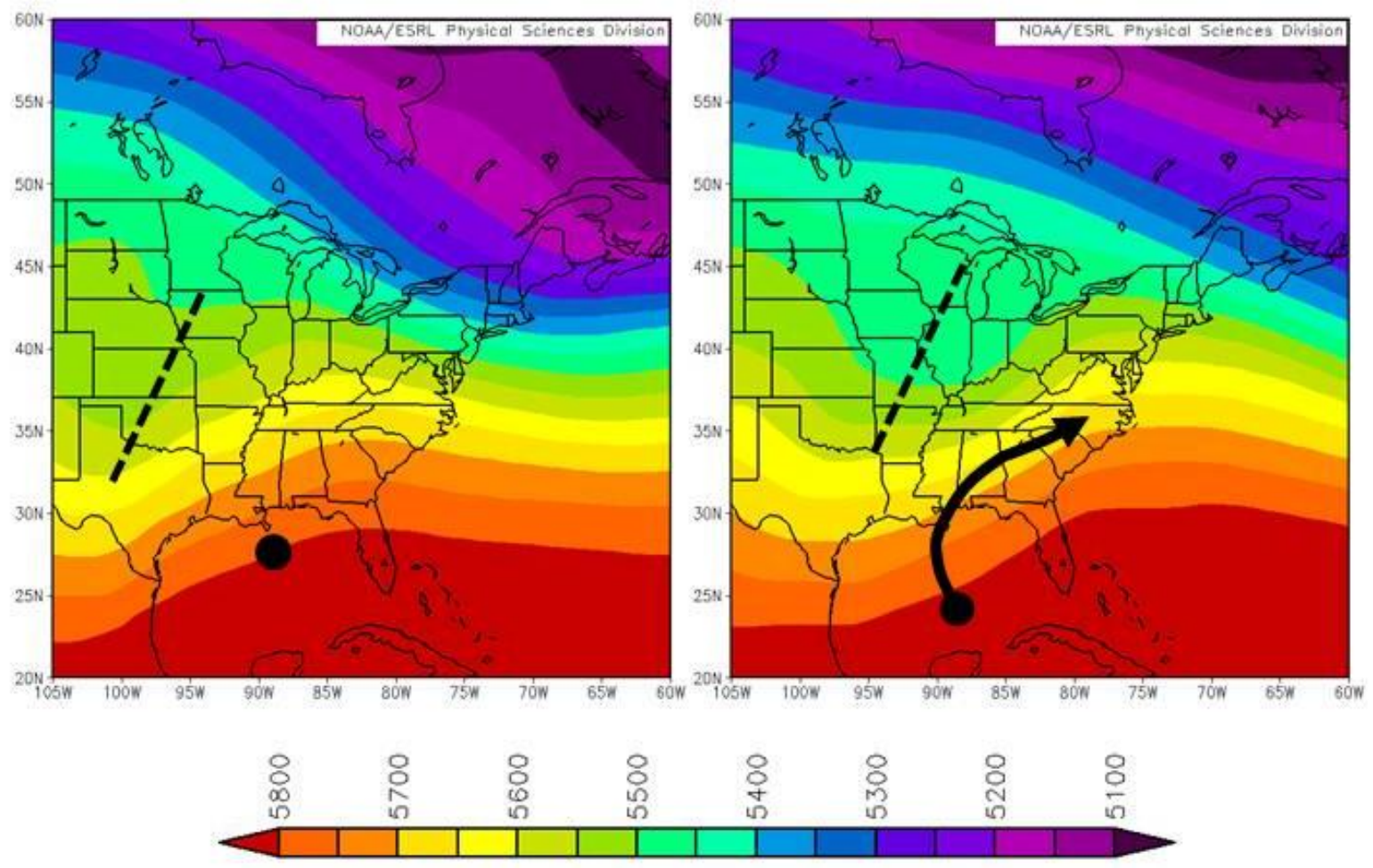

Figure 4.29. Continued 


\section{Chapter 5}

\section{DISCUSSION AND CONCLUSIONS}

\subsection{Overview}

This dissertation employed a trajectory approach to analyze the ingredients (i.e. temperature, moisture, and lift) associated with heavy snowstorms and ice storms in central North Carolina from 2000 to 2010. NOAA's Air Resource Laboratory's HYSPLIT tool was used to calculate 72-hr backward (i.e. upstream) air parcel trajectories from three critical vertical pressure levels at the time and location of heaviest precipitation during each winter storm. Analysis of composite trajectories from each vertical level revealed the source regions of various air masses as well as the contributions of adiabatic and diabatic processes to air parcel characteristics. A set of comparative and ensemble trajectories was calculated for a sample of snowstorms and ice storms to determine the trajectory characteristics and physical processes that discriminate between different precipitation types and intensities and assess the sensitivity of the trajectory calculations to the meteorological input data. Lastly, a synoptic analysis of geopotential height fields was undertaken to determine those aspects of the large-scale circulation responsible for the back-trajectory motions. In this chapter, the major findings of the dissertation are summarized and discussed. Comparisons are made between the composite trajectories revealed in this work and the traditional conveyor belt model commonly used to explain the airflow configuration of mid-latitude precipitation systems. Possible avenues for future research are also presented. 


\subsection{Major Findings}

This study demonstrates that back trajectories are useful in assessing the evolution of the ingredients and physical processes that come together to produce a winter storm. Through an analysis of the space-time character of the back trajectories, this study ties together: 1) the vertical profiles of temperature, moisture, and vertical velocity that produce winter precipitation; 2) the physical processes associated with winter precipitation; and 3) the antecedent synoptic-scale circulation responsible for the back trajectories. This study reveals that the details of the back trajectory motions and air parcel characteristics often have a significant influence on the intensity of the precipitation as well as the precipitation type (e.g. snow vs. freezing rain). Also, these details in many cases can be tied to subtle differences in the evolution of the antecedent synoptic-scale circulation.

\subsubsection{Vertical Profile}

A comparison of snowstorms and ice storms on the basis of the modeled vertical profiles of temperature, moisture, and vertical velocity revealed the following:

- Temperatures in the WLZ were above freezing in each of the ice storm profiles, with maximum temperatures warm enough for complete melting of hydrometeors (e.g. Bernstein 2000; Robbins and Cortinas 2002). A more modest temperature inversion was present in the WLZ in each of the heavy snowstorms (average temperature change of 0.5 to $\left.2.0^{\circ} \mathrm{C}\right)$.

- Moisture contents in the WLZ of ice storms were nearly double the amounts observed in the snowstorm cases. This may be tied to the presence of warmer temperatures (i.e. greater saturation mixing ratio) and greater moisture advection. Moreover, all of 
the ice storm cases displayed greater vertical velocities in the WLZ (-3.3 and -5.1 $\mathrm{cm} / \mathrm{s})$ as compared to the snowstorm cases $(-1.6$ to $-3.2 \mathrm{~cm} / \mathrm{s})$. This suggests that additional moisture was converted to precipitation in the WLZ in the ice storm cases and contributed to locally heavier precipitation rates (maximum rate of $0.22 \mathrm{in} / \mathrm{hr}$ compared to $0.12 \mathrm{in} / \mathrm{hr})$.

- Vertical velocities in the DGZ were stronger in the snowstorm cases compared to those observed in the ice storm cases (average of $-11.5 \mathrm{~cm} / \mathrm{s}$ compared to $-8.6 \mathrm{~cm} / \mathrm{s}$ ). The strong dynamical lifting observed in the snowstorm cases was tied to strong cyclogenesis off of the Southeast coast, while the ice storm cases were tied to a less dynamic synoptic regime with more gradual isentropic lifting (e.g. lower-tropospheric moisture and warm-air advection over a wedge of cold air).

\subsubsection{Trajectories}

\subsection{2a. Near-Surface Trajectory}

Trajectories associated with the near-surface level revealed the circulation within the low-level cold wedge that is typically present during winter precipitation in the region. The snowstorms and ice storms examined in this study showed qualitatively similar near-surface trajectories characterized by anticyclonic curvature and modest descent with origins over the Great Lakes region. Results of the ensemble analyses revealed that the warmer near-surface parcels (e.g. $>0^{\circ} \mathrm{C}$ ) traveled in the vicinity of the Atlantic Ocean and therefore may have been warmed diabatically as a result of latent heat fluxes from the ocean to the atmosphere (i.e. increase in potential temperature). 


\subsection{2b. Warm Layer Zone Trajectory}

One of the major goals of this research was to determine the processes and upstream features that contribute to WLZ temperatures. Typically, above freezing temperatures in the WLZ are associated with freezing rain, while below freezing temperature are associated with snow. Therefore, winter precipitation forecasts involving frozen or freezing precipitation require an accurate diagnosis of temperatures in the WLZ. A comparison of heavy snowstorms and ice storms on the basis of the WLZ trajectory revealed the following:

- In the snowstorm cases, the WLZ trajectory approached central North Carolina from the northeast where it traveled over cool waters off of the mid-Atlantic coast at a minimum altitude of approximately $1 \mathrm{~km}$. In contrast, the WLZ trajectory in the ice storm cases approached central North Carolina from the southeast where it traveled over relatively warm waters off of the Carolina coast (e.g. Figure 5.1) at a minimum altitude of $0.2 \mathrm{~km}$.

- The depth of the marine atmospheric boundary layer (MABL) depends on the seasurface temperature and the vertical thermal gradient between the ocean and the atmosphere (Raman et al. 1986). Over the cooler waters off of the mid-Atlantic coast, the average wintertime depth of the MABL ranges between 0.2 and $0.5 \mathrm{~km}$ (Raman et al. 1986). In contrast, the average wintertime depth of the MABL over the Gulf Stream ranges from $0.8 \mathrm{~km}$ along the continental shelf to $2.3 \mathrm{~km}$ along the eastern edge of the Gulf Stream (Bane and Osgood 1989). Air parcels within the MABL can induce high fluxes of sensible and latent heat from the ocean to the atmosphere, which lead to a warming and moistening of the parcels as they enter the region of precipitation. As revealed in this study, the temperature of the WLZ is 
related to the amount of diabatic warming resulting from sensible and latent heat fluxes over the course of the trajectory period.

- In the snowstorm cases, the WLZ trajectory likely remained above the MABL (> $1 \mathrm{~km})$ and therefore induced little if any sensible and latent heat fluxes. Indeed, examination of air parcel temperature, moisture content, and potential temperature along the WLZ trajectory revealed little influence from the MABL. Examination of the observed temperature and adiabatic temperature along the WLZ trajectory showed a net diabatic cooling over the final 6 hours of the trajectory. In some cases, the resulting WLZ temperature would have been too warm for snow in the absence of diabatic cooling. The exact process responsible for this cooling was not determined. One possibility is latent heat absorption due to evaporation and/or melting of falling snowflakes, which can counteract the warming due to adiabatic processes and aid in lowering the freezing level so that snow can reach the surface (Bell and Bosart 1988).

- In the ice storm cases, the WLZ trajectory descended well within the MABL $(<0.2$ $\mathrm{km}$ ) and therefore induced high fluxes of sensible and latent heat from the ocean to the atmosphere. Examination of WLZ trajectory characteristics revealed an abrupt increase in moisture, an increase in potential temperature, and a warming of air parcels up to $8^{\circ} \mathrm{C}$. Unlike the snowstorm cases, diabatic processes contributed most significantly to the warming of the WLZ over the course of the trajectory period (Figure 5.2). Examination of the observed temperature and adiabatic temperature along the WLZ trajectory revealed that air in the WLZ would have been below freezing in the absence of diabatic warming. Therefore, sensible and latent heat fluxes within the MABL off of the Carolina coast may be direct contributors to the 
occurrence of ice storms in central North Carolina. The basis for this conclusion is provided by Gyakum and Roebber (2001). Their study of the infamous ice storm of 1998 over southeast Canada revealed that air parcels connected with the heavy freezing rain spent considerable time in the Atlantic MABL where sensible and latent heat fluxes contributed to warmer parcel temperatures and higher moisture contents compared to the closest historical circulation analogs.

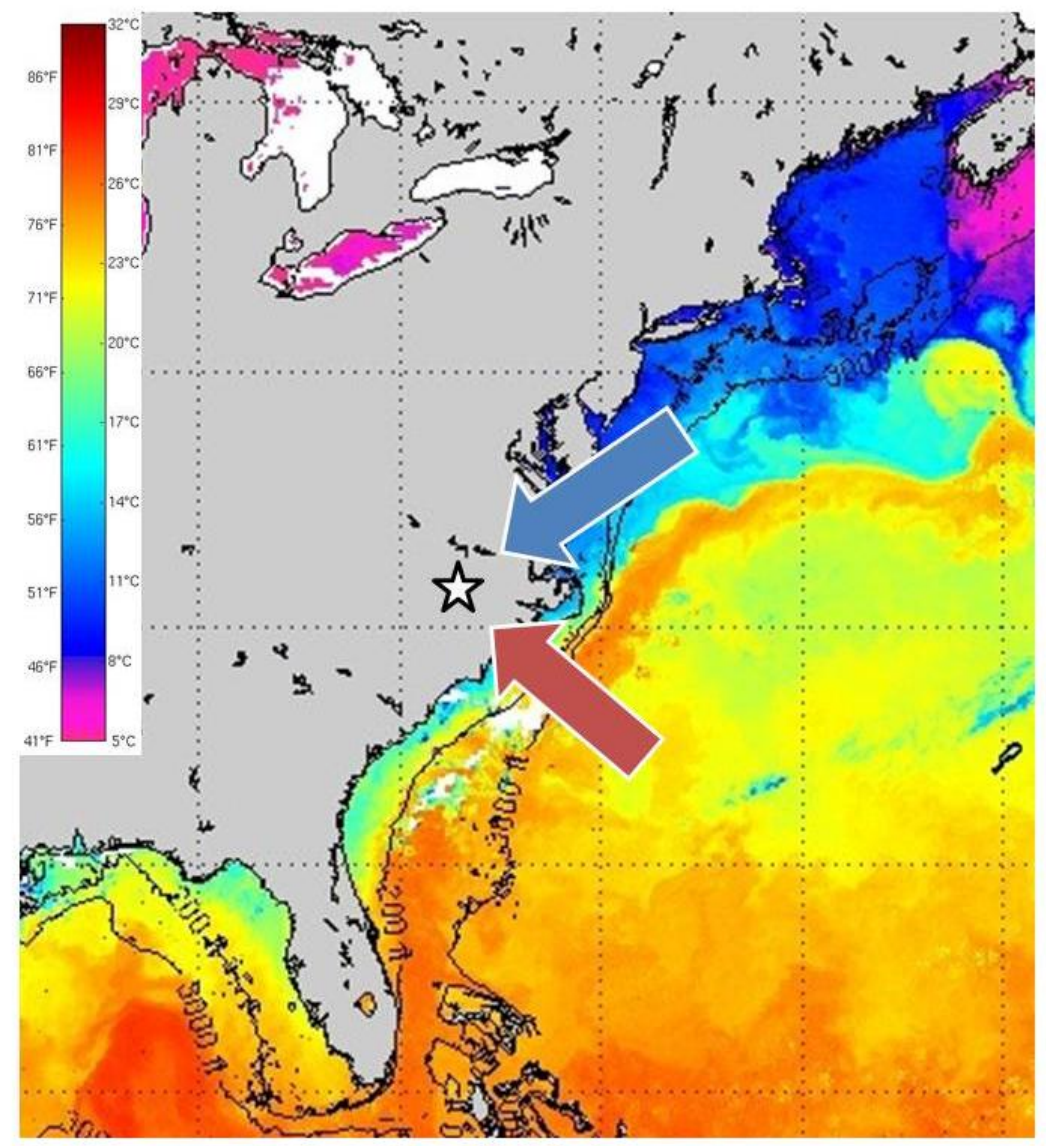

Figure 5.1. Daily sea-surface temperature composite for 7 January 2007 as measured from the NOAA-16 satellite. This image is qualitatively similar to the climatological East Coast sea-surface temperature pattern for the winter season. Arrows indicate the composite trajectory paths of air parcels terminating in the WLZ of heavy snowstorms (blue arrow) and ice storms (red arrow). Satellite imagery available from the Rutgers University Coastal Ocean Observation Lab: http://marine.rutgers.edu/mrs/sat_data/?product=sst\&nothumbs=1 


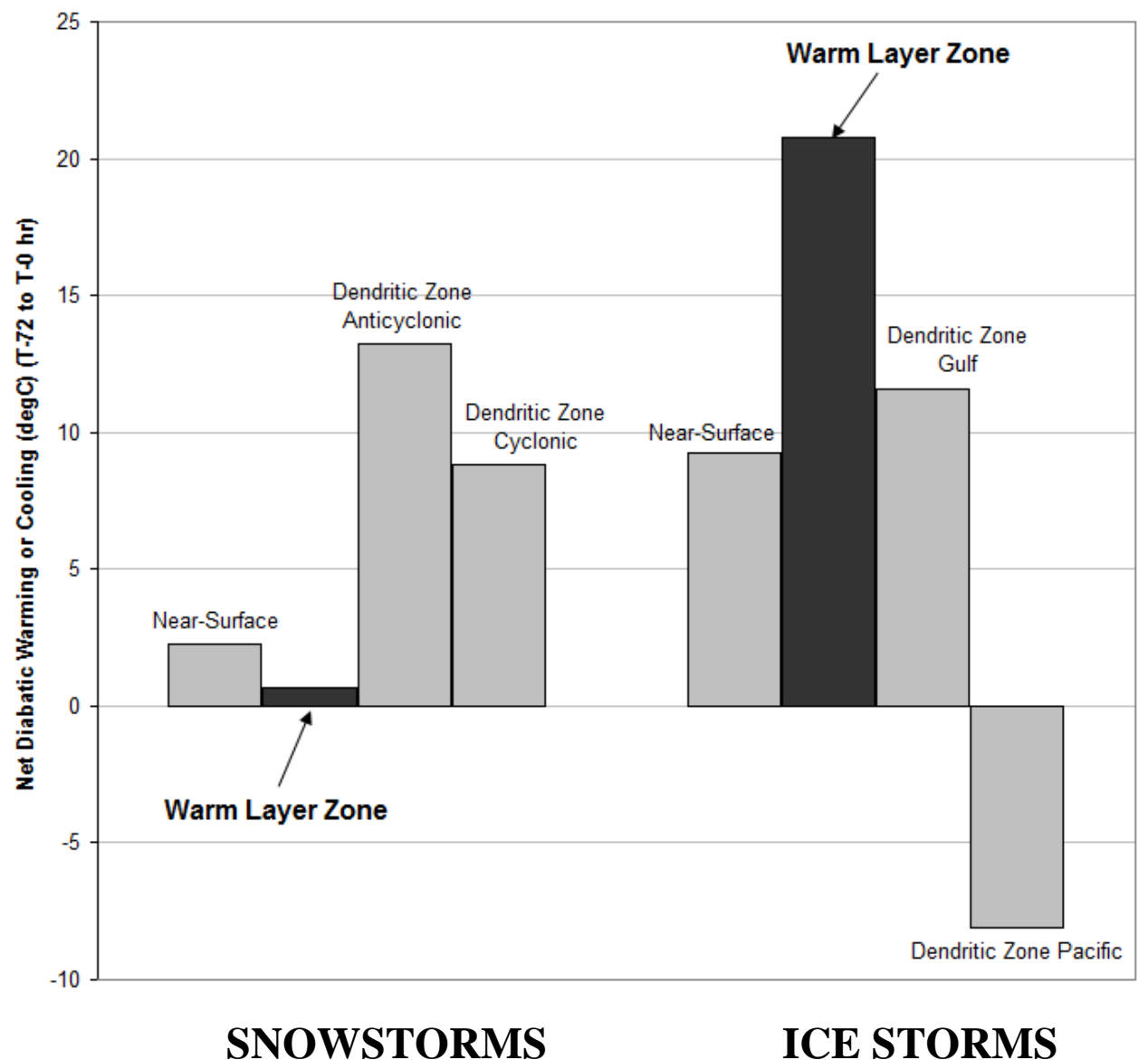

Figure 5.2. Net contributions of diabatic processes to air parcel temperature at each critical vertical pressure level over the course of the 72 -hr trajectory period. Positive (negative) values indicate a net diabatic warming (cooling).

\subsection{2c. Dendritic Growth Zone Trajectory}

Trajectories associated with the DGZ revealed the properties of the air at the level in which cold cloud precipitation production is maximized (i.e. ice crystal formation and growth is greatest). In the snowstorm cases, the DGZ trajectory exhibited two distinct paths: an anticyclonic path originating near Lake Huron and a cyclonic path originating over the Caribbean Sea. In the ice storm cases, the DGZ trajectory also exhibited two distinct paths: 
one originating over the Pacific Ocean at high altitude $(>3 \mathrm{~km})$ and one originating in the MABL over the Gulf of Mexico. These diverging paths did not show any relationship with the height of the DGZ.

The variability in the space-time character of the DGZ trajectory resulted in varying meteorological properties and, therefore, varying precipitation rates. A comparison of snowstorms on the basis of the DGZ trajectory revealed the following:

- Snowstorms with a DGZ-cyclonic trajectory originated within the MABL $(<1 \mathrm{~km})$ over the warm waters of the Caribbean Sea. These trajectories exhibited large overall moisture contents, strong vertical velocities, and well-defined evaporationprecipitation cycles. This implies high rates of condensation and deposition (i.e. precipitation formation and growth).

- Conversely, snowstorms with a DGZ-anticyclonic trajectory originated near Lake Huron and traveled southward across the Northeast U.S. before re-curving over the Atlantic Ocean. These trajectories also exhibited strong vertical velocities and welldefined evaporation-precipitation cycles, but transported significantly less moisture into central North Carolina. As a result, the maximum precipitation rates were generally lower.

- Results of the comparative trajectory and ensemble analyses revealed that snowfall intensity across central North Carolina may be tied to the characteristics of the DGZ trajectory. Air parcels within the MABL for a longer period of time (i.e. DGZcyclonic trajectories) exhibited higher moisture contents. Moreover, air parcels originating in tropical regions (below $25^{\circ} \mathrm{N}$ latitude) were able to transport nearly twice as much moisture into central North Carolina than those originating in the Gulf 
of Mexico, and more than four times as much moisture than those traveling off of the Northeast and mid-Atlantic coasts. In addition, locations experiencing the heaviest snowfall typically exhibited stronger vertical velocities in the DGZ than locations with lighter snowfall totals.

A comparison of ice storms on the basis of the DGZ trajectory revealed the following:

- The DGZ-Gulf trajectory originated within the MABL over the Gulf of Mexico. These trajectories exhibited large overall moisture contents, modest vertical velocities associated with isentropic lifting, and well-defined evaporation-precipitation cycles.

- Conversely, the DGZ-Pacific trajectory originated above the MABL (> $3 \mathrm{~km}$ ) over the eastern Pacific Ocean near the west coast of Mexico. As a result, these trajectories transported less moisture into central North Carolina. Maximum precipitation rates were, therefore, significantly lower.

- Results of the comparative trajectory and ensemble analyses revealed that freezing rain intensity across central North Carolina was higher when DGZ trajectories moved through the MABL. Specifically, air parcels that descended below $2 \mathrm{~km}$ over the Gulf of Mexico exhibited more than double the amount of moisture observed in air parcels that remained above $2 \mathrm{~km}$.

- Compared to the snowstorm cases, ice storms exhibited lower maximum moisture contents and vertical velocities in the DGZ but higher overall maximum precipitation rates. This may be explained through examination of the WLZ trajectories. Ice storms exhibited a decrease in moisture content and increase in upward vertical velocity over the final 6 hours of the trajectory. This suggests that some of moisture 
in the WLZ was converted to rain droplets (i.e. warm cloud precipitation formation) and seeded by falling hydrometeors from the DGZ above (i.e. cold cloud precipitation formation). The combined influence of warm cloud and cold cloud precipitation formation processes could explain the higher maximum precipitation rates in these cases (Stewart 1992).

\subsubsection{Synoptic Circulation}

The results of the synoptic analysis revealed that subtle differences in the large-scale circulation fields and trajectory levels can lead to significant changes in trajectory motion and air parcel characteristics. An examination of synoptic-scale circulation patterns and back trajectories associated with the snowstorm cases revealed the following:

- The near-surface trajectory originated along the eastern periphery of a surface anticyclone. The modest descent along the periphery of the anticyclone limited the amount of adiabatic warming, resulting in air parcel temperatures sufficiently cold for winter precipitation.

- The WLZ trajectory exhibited a sharp southwestward turn that corresponded to a deepening $850 \mathrm{hPa}$ trough and intensifying surface cyclone moving out of the Gulf of Mexico and up the East Coast. The WLZ trajectory continued turning cyclonically towards central North Carolina in response to the amplification and development of the upstream trough and cyclone.

- The most distinguishing characteristic of the synoptic-scale circulation with respect to the DGZ trajectory was the development and intensification of the surface cyclone. Strong cyclones characterized by rapid development and intensification were able to 
draw warm, moist air from tropical latitudes into the DGZ (i.e. DGZ-cyclonic trajectories), while weaker, early-developing cyclones advected air from northern latitudes with significantly less moisture (i.e. DGZ-anticyclonic trajectories).

An examination of the ice storm cases revealed the following:

- As in the snowstorm cases, the near-surface trajectory originated along the eastern periphery of a surface anticyclone with a well-defined CAD signature. The anticyclone in the ice storm composite was stronger than in the snowstorm composite, which supports the findings of Cuviello (2007) and likely explains why the initial temperature of the near-surface trajectory was colder in the ice storm composite.

- The anticyclonic curvature of the WLZ trajectory coincided with the amplification of an $850 \mathrm{hPa}$ trough into a closed low over the Midwest. The modest ascent profile of the WLZ trajectory over the last 24 hours is indicative of isentropic lifting associated with a less dynamic synoptic regime.

- The most distinguishing characteristics of the synoptic-scale circulation with respect to the DGZ trajectory were the pattern of cyclogenesis and amplitude of upstream short-wave toughs. DGZ-Gulf trajectories were associated with higher-amplitude short-wave troughs at 850 and $500 \mathrm{hPa}$ (i.e. increased southerly component to the large-scale flow) and secondary cyclogenesis along the Southeast coast. In contrast, DGZ-Pacific trajectories were associated with relatively weaker troughs over the Midwest (i.e. more westerly component to the large-scale flow) with a primary cyclone located southwest of the study area. 


\subsection{Comparisons to the Conveyor Belt Model}

The conveyor belt model (section 1.2.5) offers a three-dimensional conceptual perspective on the airflow characteristics of mid-latitude weather systems. In recent years, refinements to this model have been made by Bierly (1999), Bierly and Winkler (2001), Schultz (2001), Ng (2005), along with several others, in an attempt to clarify the connections between the storm-relative airflow of mid-latitude cyclones and the details of the resulting precipitation patterns. In a similar vein, comparisons are made here between the composite trajectories presented in this study and the main belts of air described in the traditional conveyor belt model (Figures 1.9 and 1.10).

The conveyer belt model represents storm-relative airflow that remains above 950 $\mathrm{hPa}(\mathrm{Ng} 2005)$. The near-surface trajectories occur immediately below this airflow in an anticyclonic path from north to south into the region of cold-air damming (CAD). The cold conveyer belt (CCB), an easterly flow ahead of and beneath the warm front (i.e. WCB), would be positioned immediately above the near surface air trajectory and the CAD. Previous studies have shown the CCB to be an important contributor of Atlantic moisture to heavy precipitation events in the eastern and Midwest U.S. (e.g. Liu 1997; Nicosia and Grumm 1999; Moore et al. 2005).

The WLZ and DGZ trajectories identified in this study bear close resemblance to various characteristics of the CCB and WCB. For instance, the WLZ trajectory in the snowstorm shares some similarities to the $\mathrm{CCB}$, which travels beneath the $\mathrm{WCB}$, exhibits minimal vertical motion, and transports greater amounts of moisture into the region of precipitation (Schultz 2001; Moore et al. 2005). Additionally, $\mathrm{Ng}$ (2005) found that the CCB associated with two Midwestern cyclones extended up to $838 \mathrm{hPa}$, which is near the level of 
the WLZ (850 hPa). However, the lack of reduction in moisture over the final segments of the WLZ trajectory implies that this air does not contribute directly to the production of precipitation (i.e. water vapor is not converted to precipitation). Instead, a unique aspect of the WLZ trajectory is the occurrence of diabatic cooling associated with evaporation and sublimation of precipitation over the final segment of the trajectory. This suggests that the primary role of the WLZ trajectory is to maintain a temperature in the lower troposphere (i.e. below $850 \mathrm{hPa}$ ) supportive of snowfall.

By comparison, the WLZ trajectory in the ice storm cases bears a close resemblance to a form of the CCB described by Bierly (1999) and Bierly and Winkler (2001) as the cyclonically-turning moist airstream, or CMA (Figure 5.3). During the developing stages of a sample of central U.S. cyclones, the CMA was shown to originate over the Gulf of Mexico in air that was as warm and moist as the air associated with the WCB (Bierly 1999; Bierly and Winkler 2001). However, during the mature stage, the CMA was shown to originate north of the cyclone above a cold air mass and follow a path similar to the final segments of the WLZ trajectory. Since the trajectory composites in the present study were calculated at the time and location of heaviest precipitation (i.e. event maturation), it is possible that the WLZ trajectory in the ice storm cases is better understood conceptually as a CMA rather than a WCB or CCB. A unique aspect of the WLZ trajectory in the ice storm cases is that during event maturation the air descends into the MABL over the Gulf Stream waters before ascending above the near-surface trajectory.

All of the DGZ trajectories exhibit characteristics of a WCB. For example, the DGZcyclonic trajectory, which was tied to four of the eight snowstorms, originates within the MABL over the warm waters of the Caribbean Sea, advects significant amounts of heat and 
moisture into the region of precipitation, and exhibits strong vertical motions in the vicinity of a mature cyclone (Wernli 1997; Eckhardt et al. 2004; Moore et al. 2005; Ng 2005). The origin of the DGZ-cyclonic trajectory also coincides with the global maximum in starting location of WCBs, which later terminate over northern Europe (Eckhardt et al. 2004). Because composite trajectories were only examined at one point in time (i.e. event maturation), it is unknown whether the airflow associated with the DGZ-cyclonic trajectory is maintained into the high latitudes. The extension of the DGZ-cyclonic trajectory along the northwest quadrant of the surface cyclone (in the region of heavy snowfall across central North Carolina) suggests the presence of a TROWAL airstream (i.e. trough of warm air aloft; Martin 1998). In contrast, the DGZ-anticyclonic trajectory, which was tied to the remaining four snowstorms, originates in a cold air mass over the Great Lakes and descends anticyclonically into the MABL in the vicinity of the Gulf Stream. While the magnitude of vertical lift observed in the DGZ-anticyclonic trajectory is comparable to that observed in the $\mathrm{WCB}$, the space-time character and thermodynamic characteristics of the trajectory bear a stronger resemblance to the CMA described earlier.

The DGZ-Gulf trajectory, which was tied to four of the seven ice storms, originates within the MABL over the Gulf of Mexico, but unlike a traditional WCB, exhibits gradual anticyclonic ascent into the DGZ over central North Carolina. This ascent profile resembles a form of the WCB described by Bierly (1999) and Bierly and Winkler (2001) as the anticyclonically-turning moist airstream, or AMA (Figure 5.3). While the thermodynamic characteristics of the AMA are similar to the traditional $\mathrm{WCB}$, the ascent profile encompasses a broad region of isentropic lifting over the CCB/CMA, which typifies the DGZ-Gulf trajectory. In contrast, the DGZ-Pacific trajectory exhibits generally cyclonic 
curvature as it enters central North Carolina, which is chracteristic of a traditional WCB. However, the DGZ-Pacific trajectory originates well above the MABL, advects relatively little moisture, and exhibits less vertical motion than a typical WCB (Wernli 1997; Eckhardt et al. 2004; Moore et al. 2005; Ng 2005). In this case, the DGZ-Pacific trajectory may correspond to the upper boundary of a relatively deep $(\sim 3 \mathrm{~km})$ WCB.

The results of this dissertation reveal that the details of the back trajectory motions and air parcel characteristics often have a significant influence on the intensity of the precipitation as well as the precipitation type (e.g. snow vs. freezing rain). Also, these details in many cases can be tied to key differences in the evolution of the antecedent synoptic-scale circulation. As illustrated in Roebber and Bosart (1998) and Homan and Uccellini (1987), the sensible weather that results from a particular circulation pattern is highly dependent on the details of the airflow. Consequently, variations in the large-scale circulation can result in trajectories with significantly different source regions and meteorological properties and, therefore, different precipitation patterns. For example, variations in the maximum precipitation rate among the snowstorm cases were tied to the path of the DGZ trajectory and the development and intensification of an upstream surface cyclone. Other variations may be more subtle and therefore require particular attention to the details of the synoptic-scale circulation. For example, the path of the DGZ trajectory in the ice storm cases was tied to the amplitude of upstream short-wave troughs. Ice storms with a DGZ-Pacific trajectory exhibited relatively weak short-wave troughs over the central U.S., resulting in a more zonal circulation pattern. In contrast, ice storms with a DGZ-Gulf trajectory exhibited a more amplified short-wave trough pattern over the central U.S., resulting in a more southerly component to the lower and middle tropospheric flow. Additionally, ice storms with a DGZ- 
Gulf trajectory exhibited secondary cyclogenesis along the Southeast coast. These subtle differences in the synoptic regime led to deviations in the DGZ trajectories that resulted in significantly different maximum precipitation rates among the ice storm cases.

As previously mentioned, this project builds upon the methods and conceptual models for forecasting winter precipitation in central North Carolina that have been developed through a collaborative research program involving the Raleigh, NC NWS and North Carolina State University. The most recent project involving winter precipitation, which ended in 2006, focused on improving quantitative precipitation forecasts (QPF). Conceptual models were developed to illustrate the relationships between QPF, coastal cyclogenesis, upstream convection, cold-air damming, and lower-tropospheric potential vorticity (Lackmann 2006). The present study builds upon these methods by using a trajectory approach to identify the upstream features and physical processes that contribute to different precipitation types and intensities in the region.

Currently, the Air Resource Laboratory produces 36-hr forecast trajectories at 6-hr intervals using the North American Mesoscale Model. Forecasters could calculate individual meteorological parameters, including adiabatic and diabatic temperature changes, along each forecast trajectory using a gridded forecast product of their choice to determine the temperature of the near-surface and WLZ, as well as calculate rates of condensation and deposition in the DGZ. Additionally, as an event unfolds, forecasters can run multiple trajectories with up-to-date forecast model output to determine the likely precipitation type at different locations and at different stages of the event. This type of framework would require forecast models capable of adequately resolving ocean-atmosphere energy exchanges and heat fluxes within the MABL, as these were found to be important contributors to lower- 
tropospheric temperatures and moisture profiles during heavy winter storms in central North Carolina.

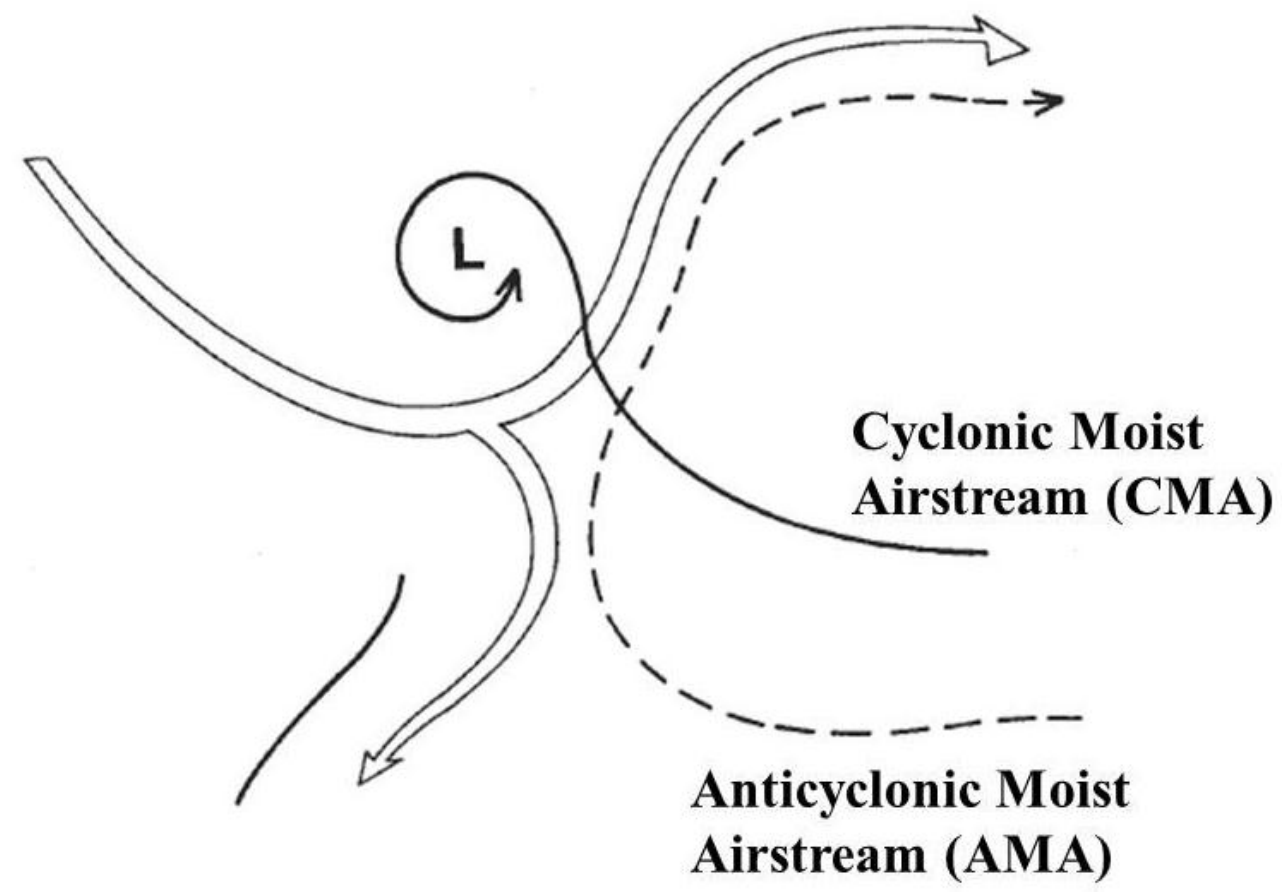

Figure 5.3. A relative-wind airflow (i.e. conveyor belt) diagram based on analysis of cold season central U.S. cyclones. The solid black line with arrow is the cyclonic moist airstream of the cold conveyor belt. The dashed line with arrow is the ascending anticyclonic moist airstream of the warm conveyor belt. From Bierly (1999), his Figure 1.

\subsection{Future Research}

Several avenues of future research are identified that build off of the results of this study or address limitations in the data and research methods.

- This study examined a small sample of events from the most recent 10 -yr period. A larger sample of events, including those with lighter precipitation amounts, could reveal the climatological significance of the trajectory characteristics identified in this 
work. Additionally, expanding the study area to include other parts of the Southeast U.S. would reveal how changes in topography and proximity to large water bodies affect the space-time character and meteorological properties of air parcels connected with winter precipitation across the region. For example, how might the trajectory characteristics differ between snowstorms in the Shenandoah Valley of Virginia and snowstorms in the inner coastal plain of North Carolina? By examining a broader range of storm types and intensities, a conceptual model could be developed that integrates the back trajectories, ingredients, and physical processes associated with winter precipitation within a synoptic-climatological framework. These conceptual models may be directly applicable to forecasters in the region as well as provide insight for studies of future winter precipitation scenarios in the region based on downscaled climate and coupled ocean-atmosphere models.

- This study used back trajectories to examine the three fundamental ingredients associated with winter precipitation: temperature, moisture, and vertical velocity (i.e. lift) (Wetzel and Martin 2001). While the rates of condensation, deposition, and evaporation were estimated through changes in the moisture content along each trajectory, the precipitation efficiency (i.e. the ratio of the amount of precipitation to the amount of water vapor in the atmospheric column), termed a secondary ingredient, was not directly measured. Moreover, the presence of instability was not assessed in the trajectory analyses. Future research should examine whether these ingredients can be directly measured using a trajectory approach.

- Additional research is needed to determine if the traditional conveyor belt model is applicable to winter precipitation events in the region. Analysis of trajectories from 
all available vertical pressures and at different times and locations over the course of an event should be undertaken. Since these atmospheric conveyor belts can extended over several degrees of latitude (Eckhardt et al. 2004), the use of global reanalysis datasets would allow the trajectories to be extended beyond $72-\mathrm{hr}$ so that remote sources of moisture, lift, and energy exchanges can be identified. A recent study by Gustafsson et al. (2010) suggests that moisture source regions over the mid-latitudes can be traced back as many as eight days.

- Future research will benefit from the use of higher resolution datasets (e.g. the $12 \mathrm{~km}$ North American Mesoscale Model) and modeling capabilities (e.g. the Weather Research and Forecasting model). In particular, higher resolution datasets and models may aid in determining the horizontal and vertical extent of the primary conveyor belts and airflow trajectories, as well as capture the influence of upstream convection on downstream winter precipitation (Mahoney and Lackmann 2007). For example, how are the space-time characteristics and meteorological properties of the air advected into central North Carolina affected by upstream convection? Does the presence of convection in the Gulf of Mexico enhance or inhibit winter precipitation over central North Carolina?

- One of the main results of this work was an assessment of the difference in vertical extent of the WLZ trajectory over the Atlantic Ocean between snowstorms and ice storms, which appeared to play a critical role in the resulting temperature profile along the trajectories. Future research is needed to determine the physical mechanism(s) that cause the WLZ trajectory in the ice storm composite to descend deeper into the MABL. Future research is also needed to determine the relationship 
between sea-surface temperature anomalies and the resulting temperature profile along the trajectories. For example, are warmer WLZ trajectories (i.e. freezing rain) associated with warmer Atlantic sea-surface temperatures (Ramos da Silva et al. 2006)? Global reanalysis datasets could also be used to directly calculate the magnitude of the sensible and latent heat fluxes that are exchanged between the ocean and the air parcels within the MABL. More generally, additional work is needed to understand and parameterize the interactions between the ocean and the air within the MABL.

- Atmospheric trajectories have been shown to be good indicators of large-scale flow regimes (Roberge et al. 2009). Therefore, it is likely that large-scale circulation indices and hemispheric teleconnections, such as the El Nino-Southern Oscillation (ENSO), the Pacific-North American (PNA) pattern, and the North Atlantic Oscillation (NAO), may be useful in predicting trajectory motions. Future research should examine the relationship between these indices and the space-time character of back trajectories associated with winter precipitation. For example, are DGZcyclonic and DGZ-Gulf trajectories, which were associated with the highest precipitation rates in this study, more common during El Nino winters? These relationships can provide a better assessment of possible precipitation patterns for medium and long-range forecasts.

- The results of this research may have important implications for studies of global climate change and its influence on winter precipitation in central North Carolina. It is possible that warmer temperatures over the source regions of the near-surface and WLZ trajectories may result in fewer snowstorms and ice storms. However, higher 
sea-surface temperatures may result in more ice storms at the expense of snowstorms (due to warmer WLZ temperatures) assuming that near-surface conditions are sufficiently cold. Future research is needed to test these hypotheses. 


\section{APPENDIX I}

Precipitation Maps

Snow Storms

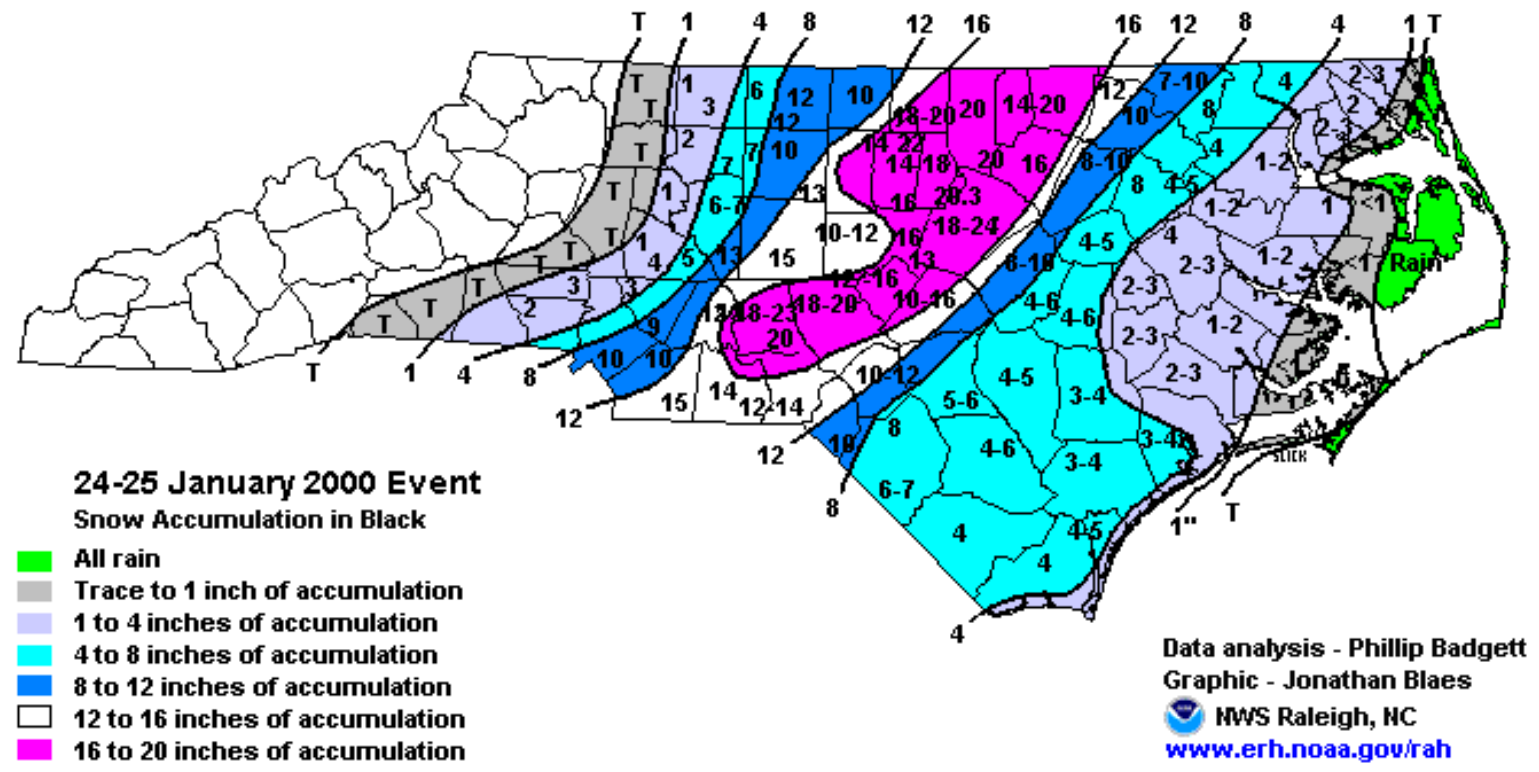

Figure A1. 24-25 January 2000

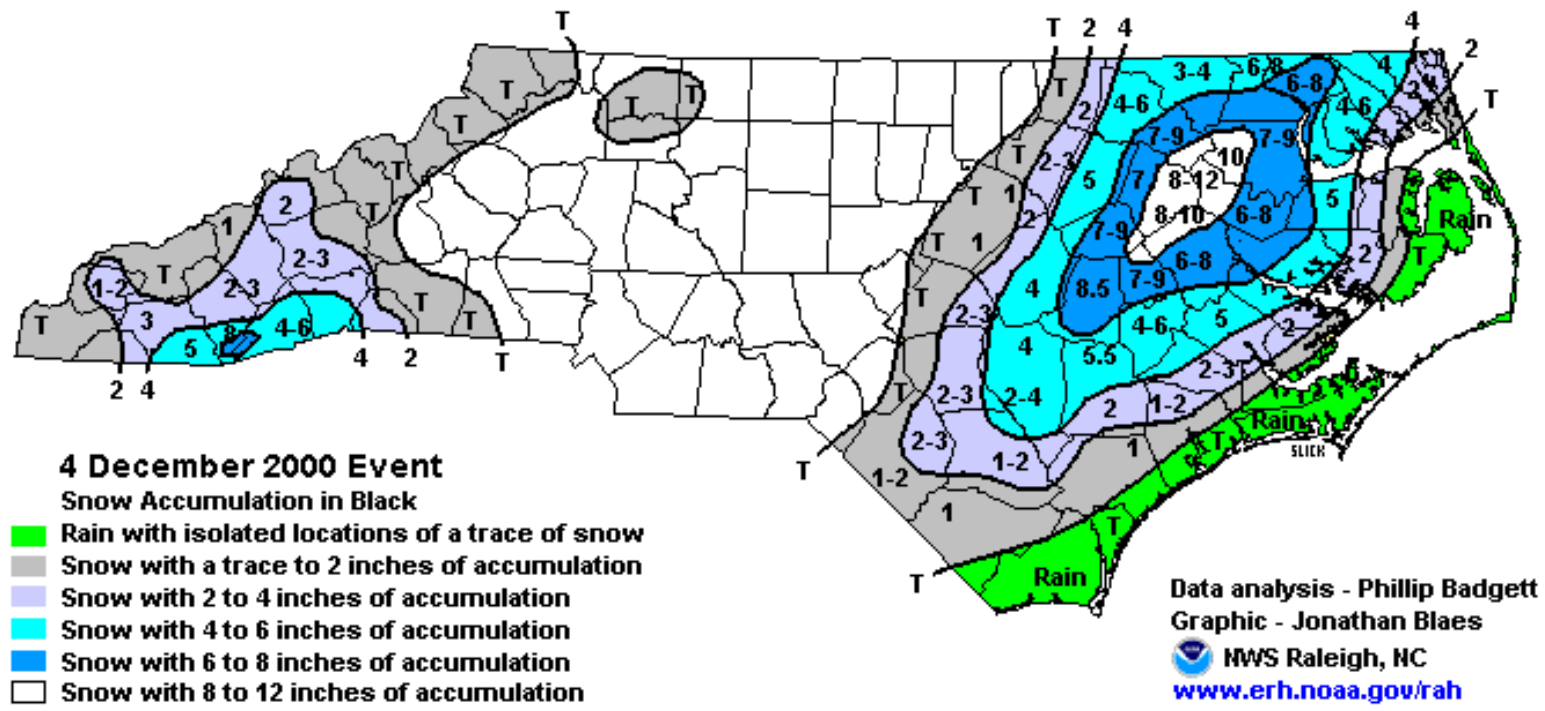

Figure A2. 3-4 December 2000 


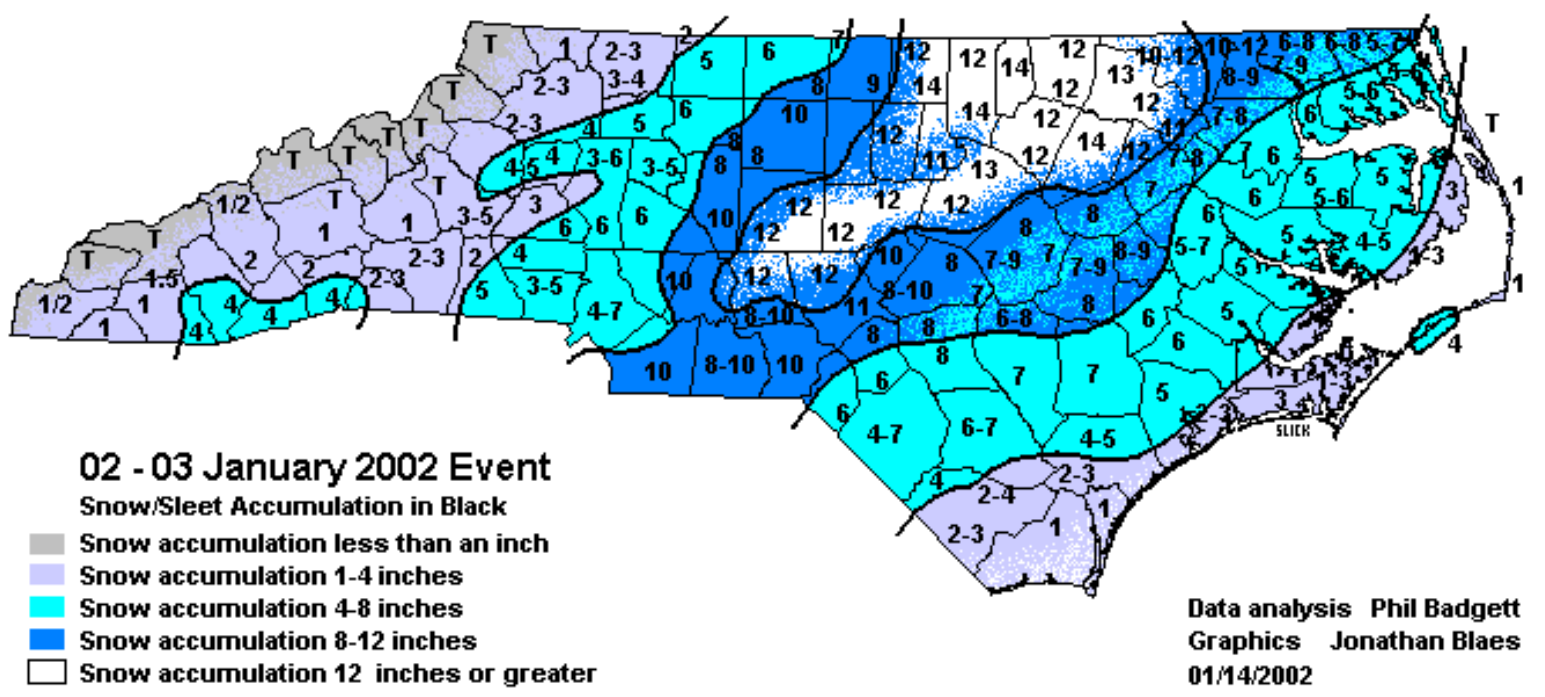

Figure A3. 3-4 January 2002

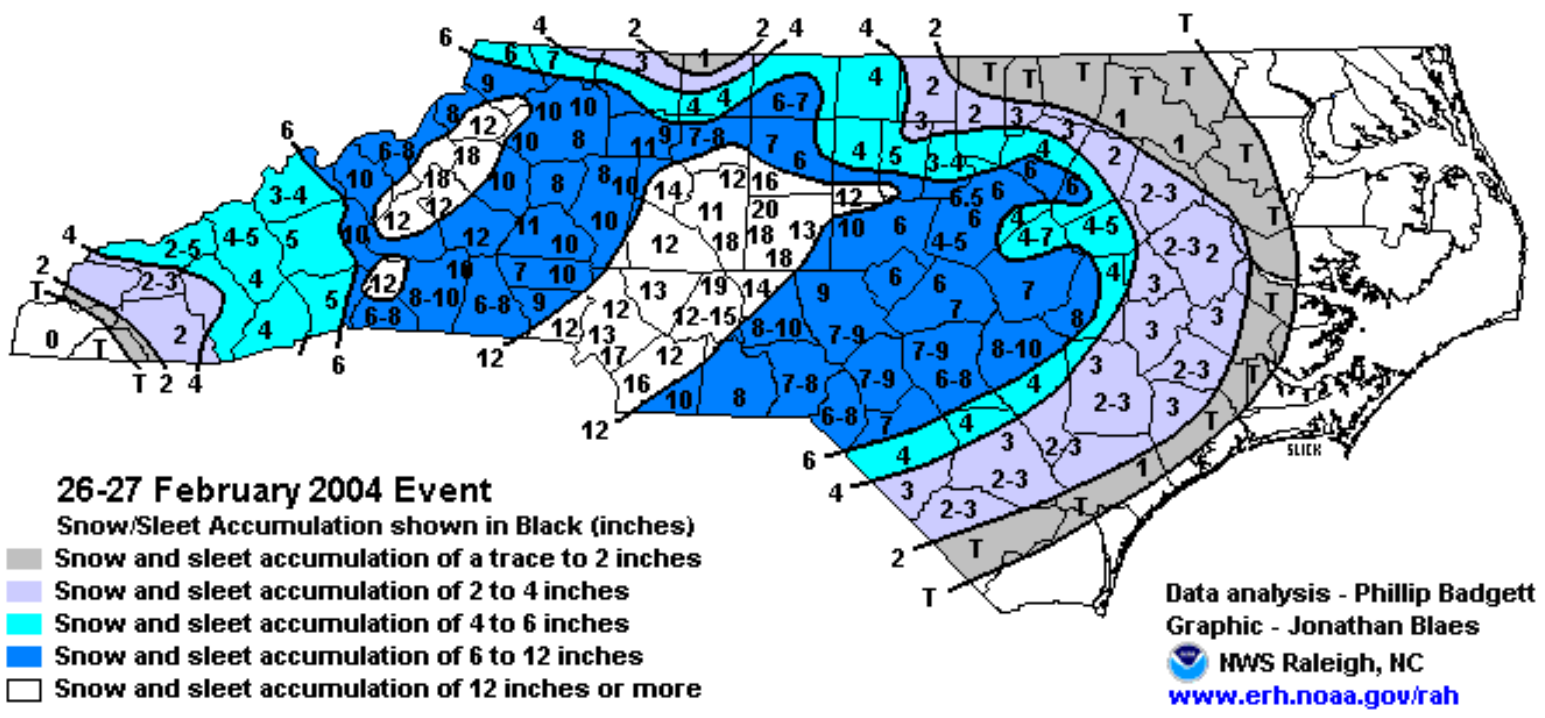

Figure A4. 26-27 February 2004 


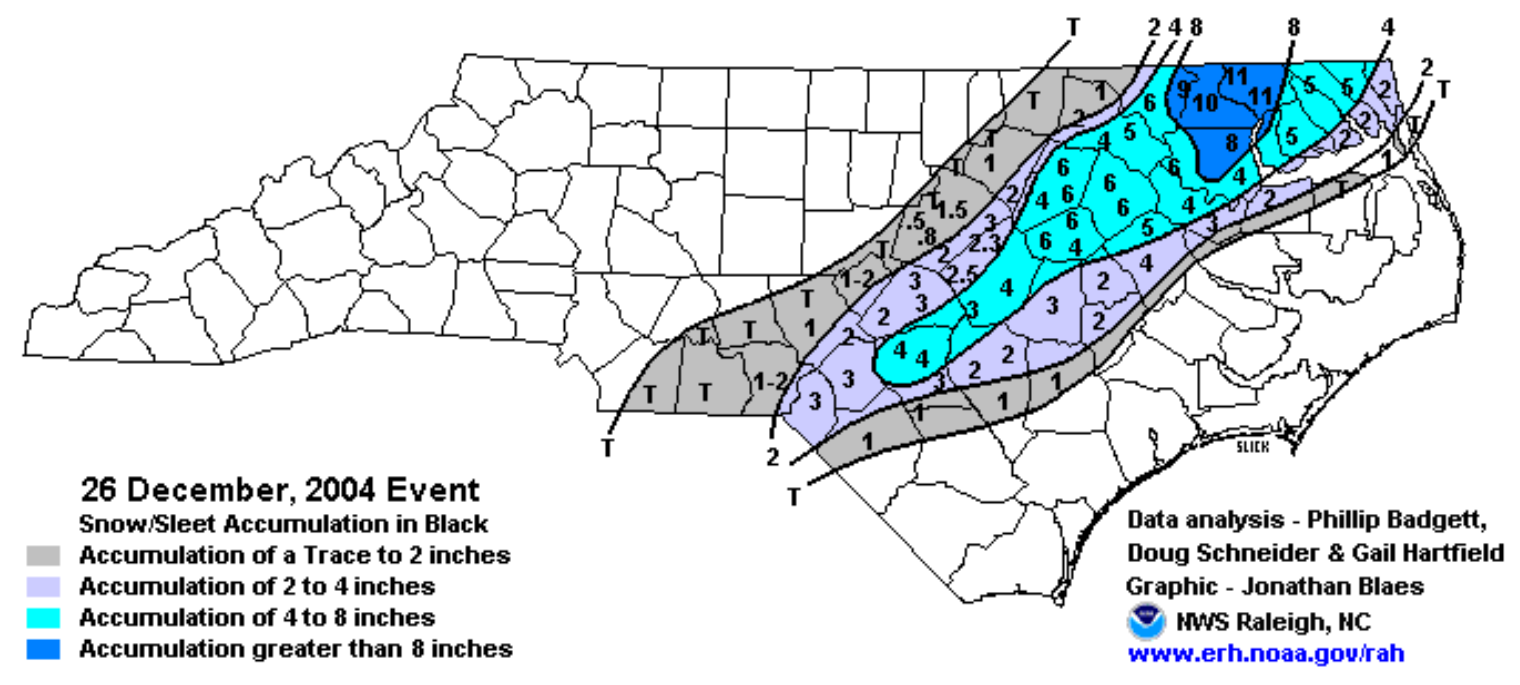

Figure A5. 26 December 2004

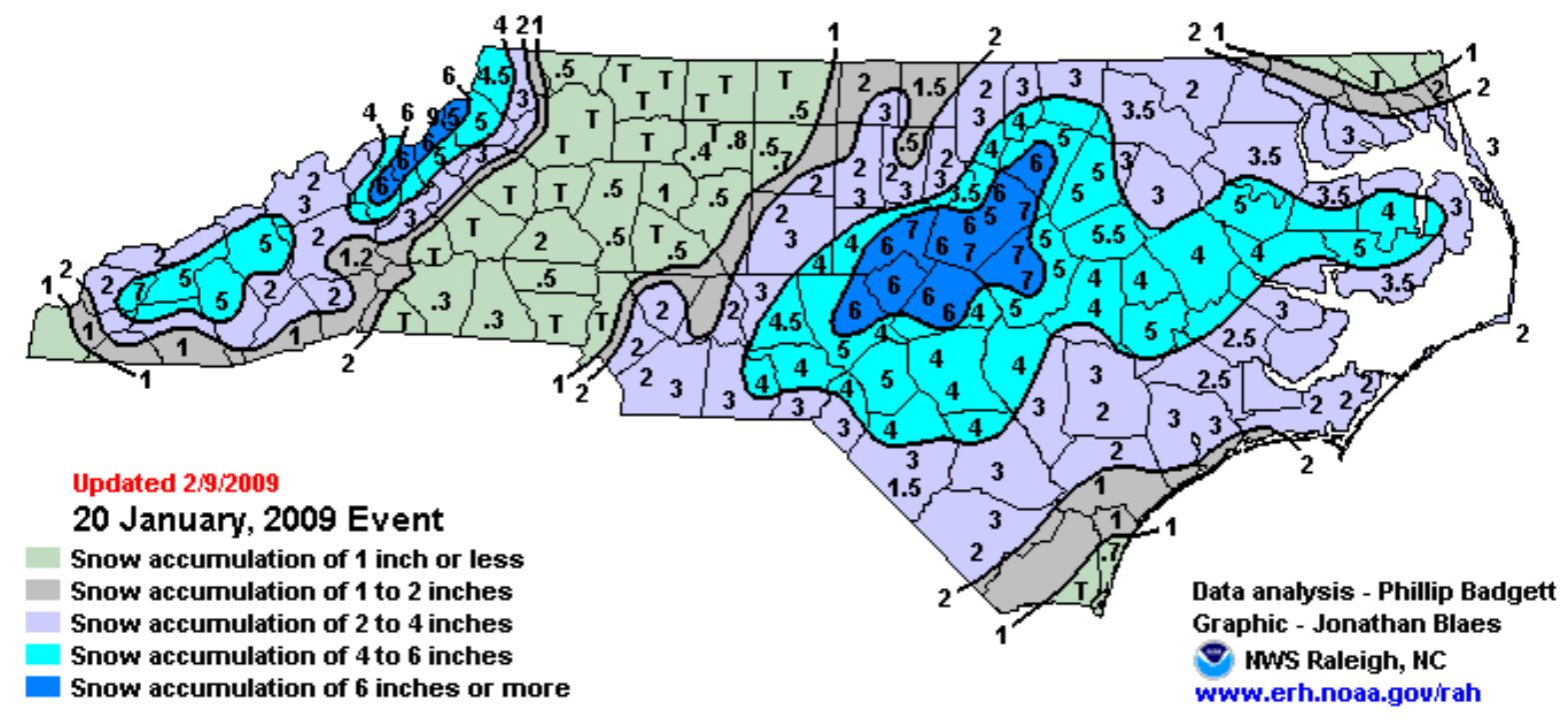

Figure A6. 20 January 2009 


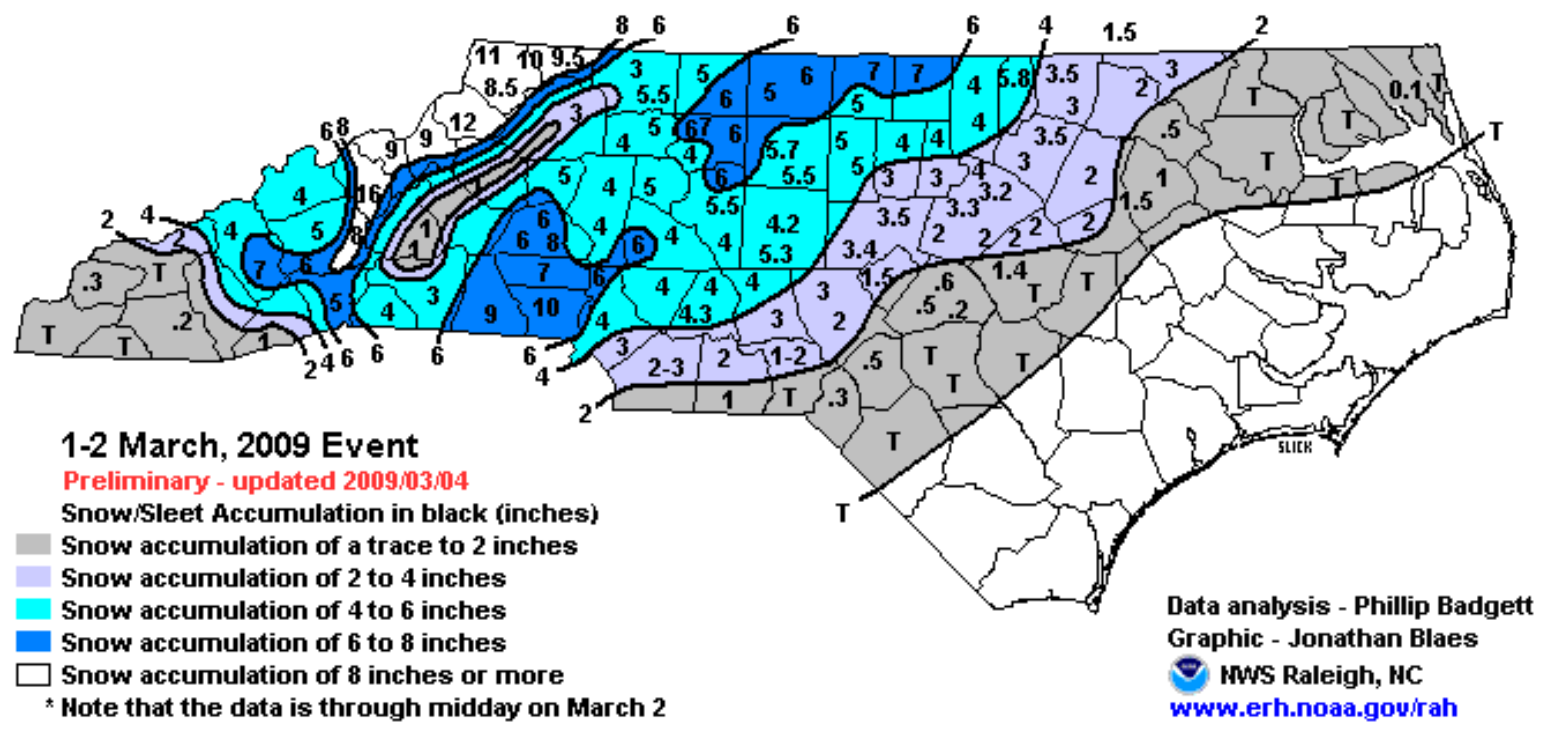

Figure A7. 1-2 March 2009

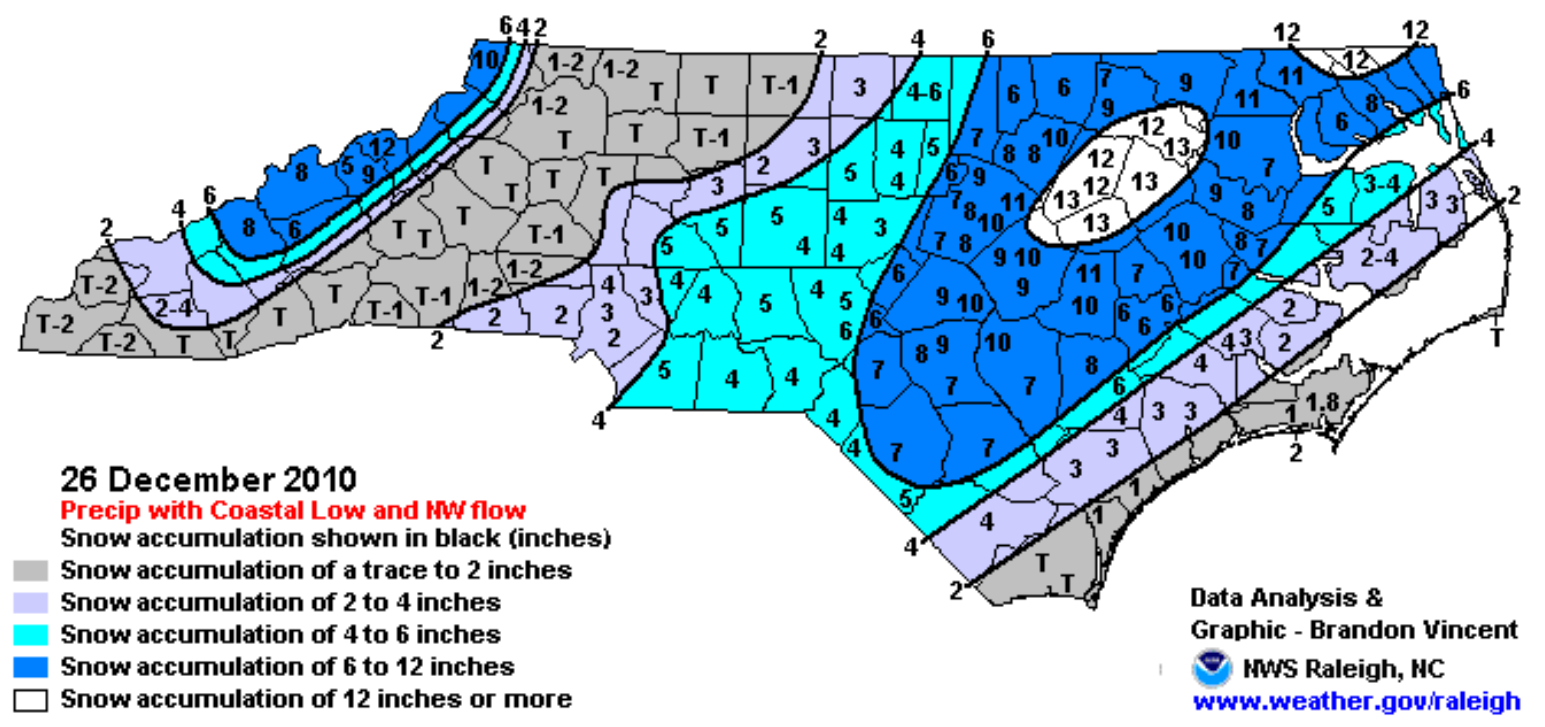

Figure A8. 26 December 2010 
Ice Storms

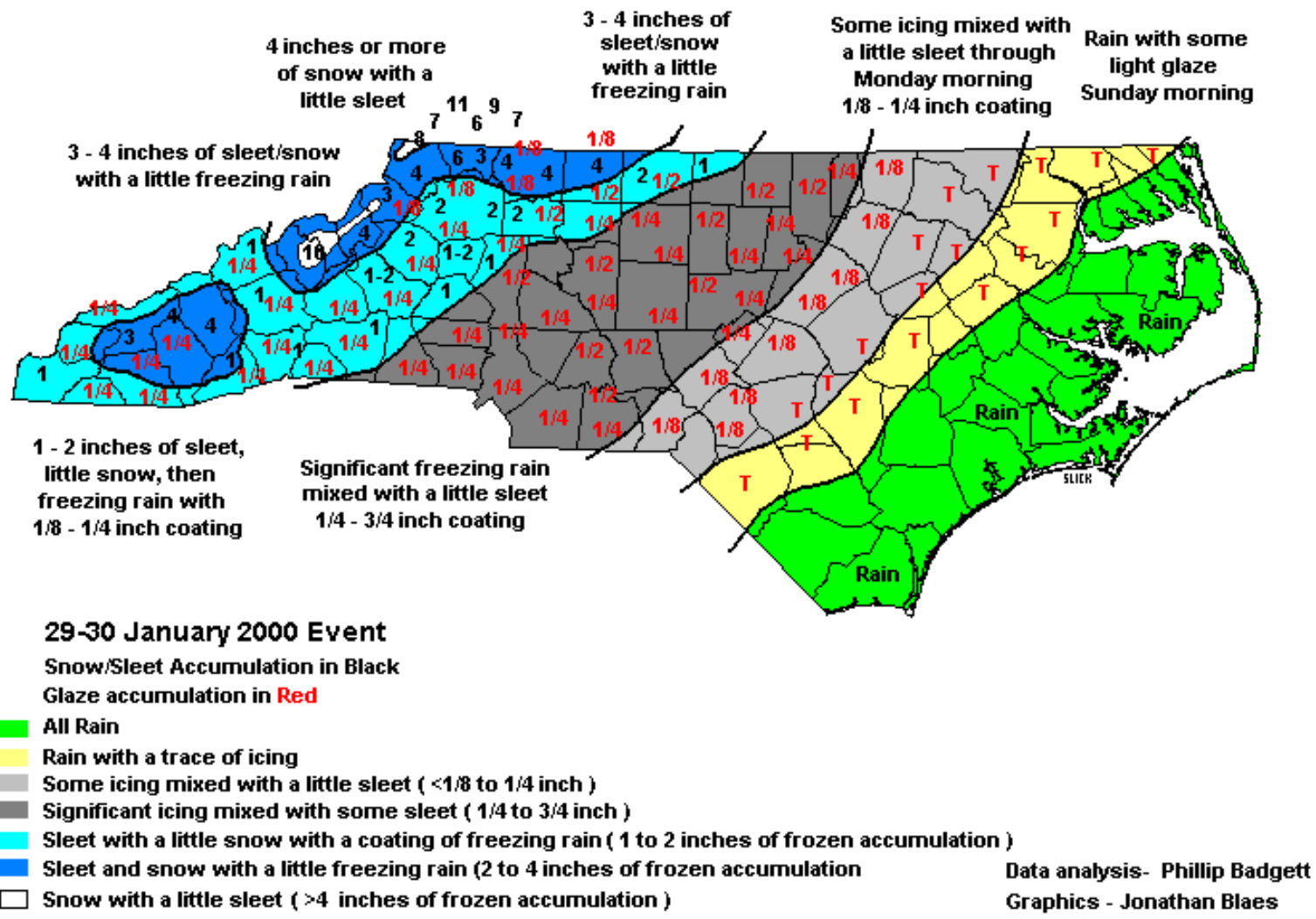

Figure A9. 30 January 2000 


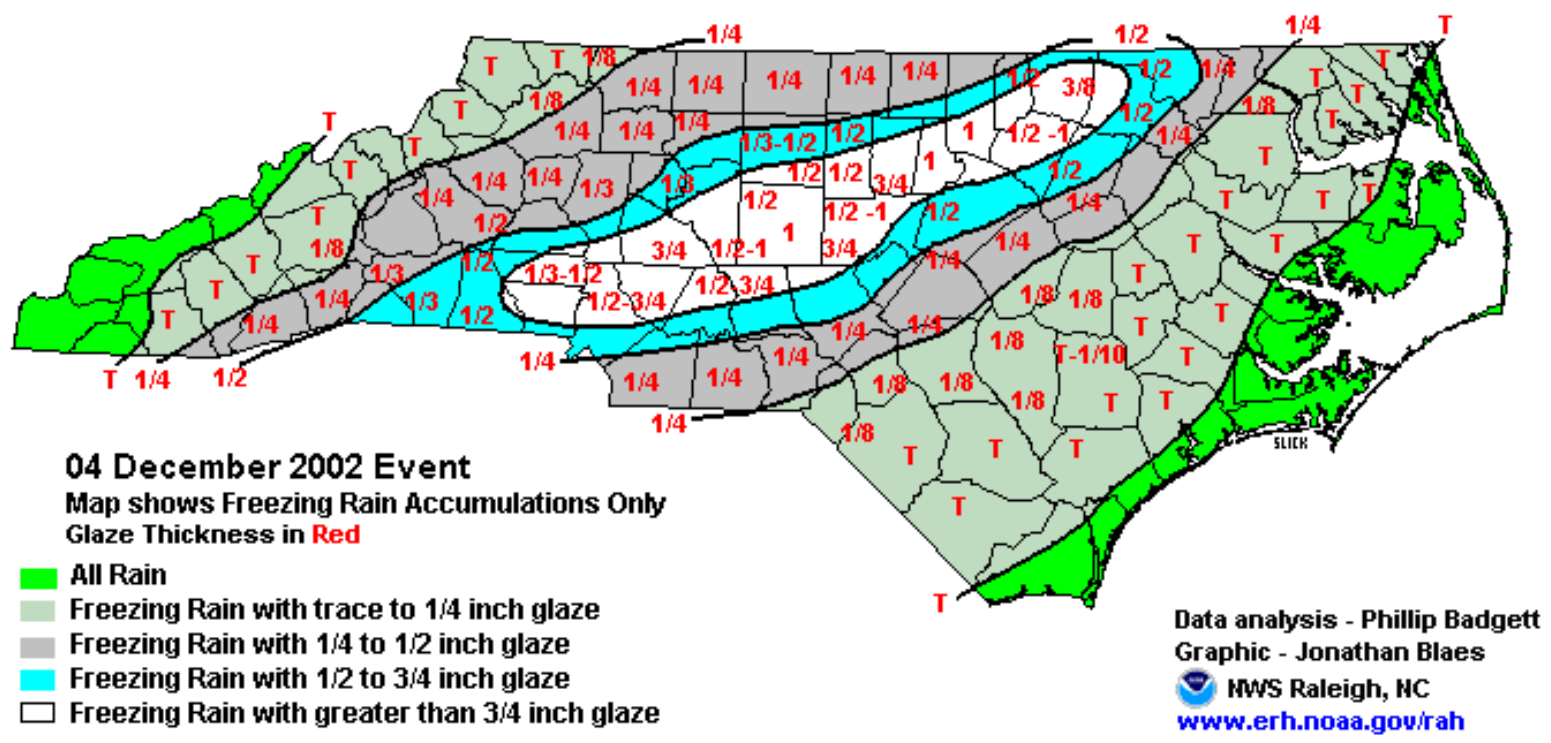

Figure A10. 4-5 December 2002

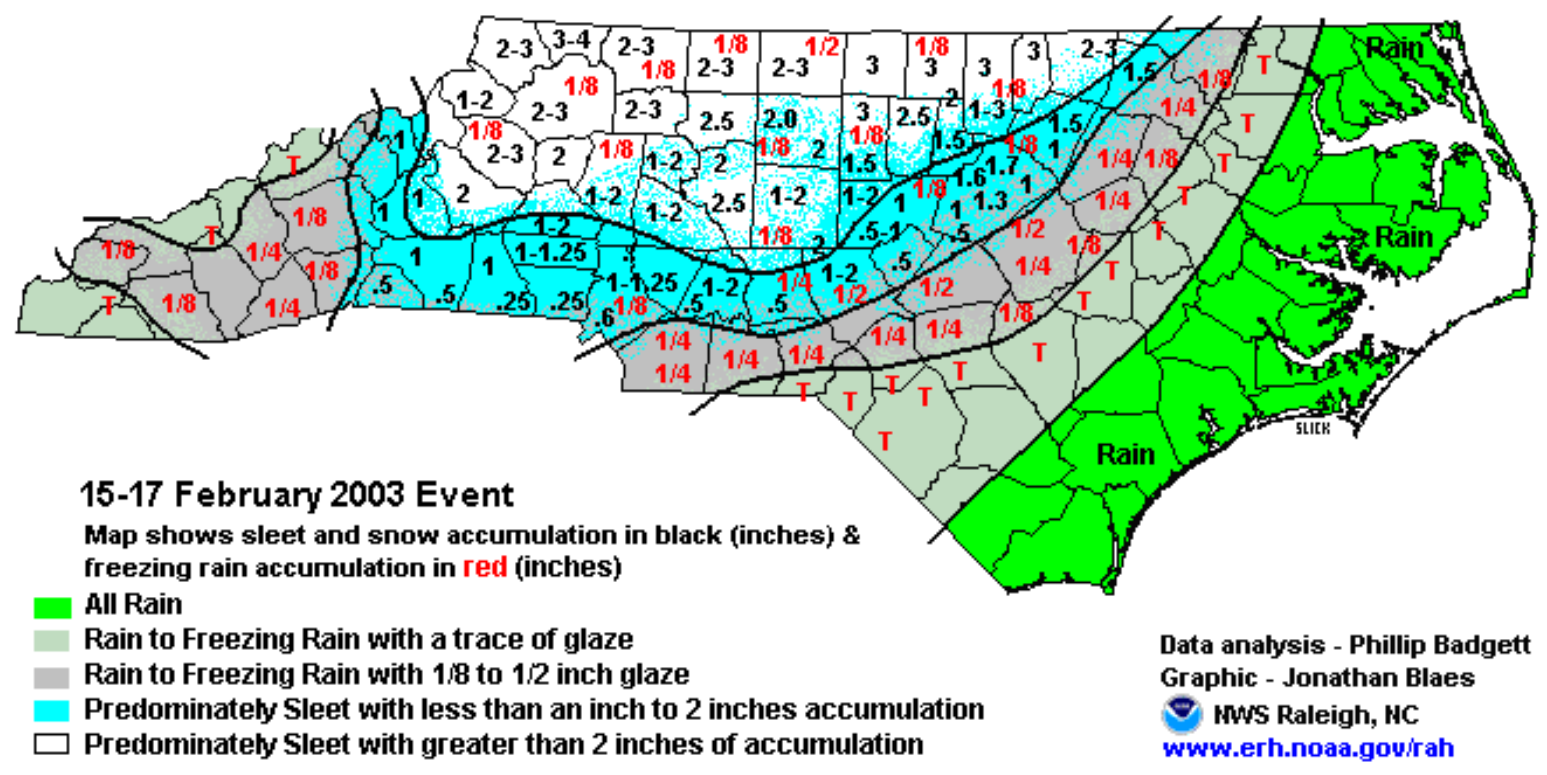

Figure A11. 16-17 February 2003 


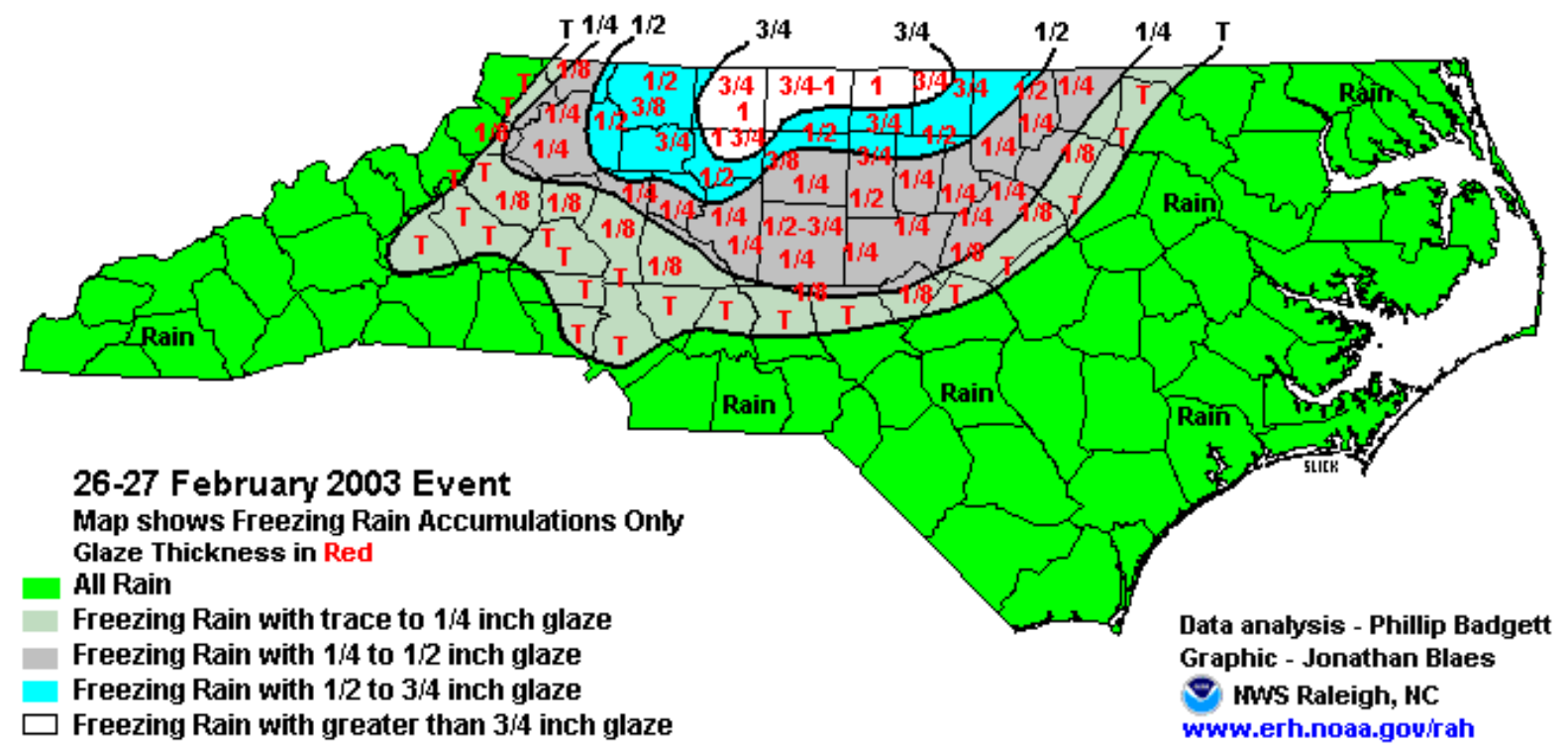

Figure A12. 26-27 February 2003

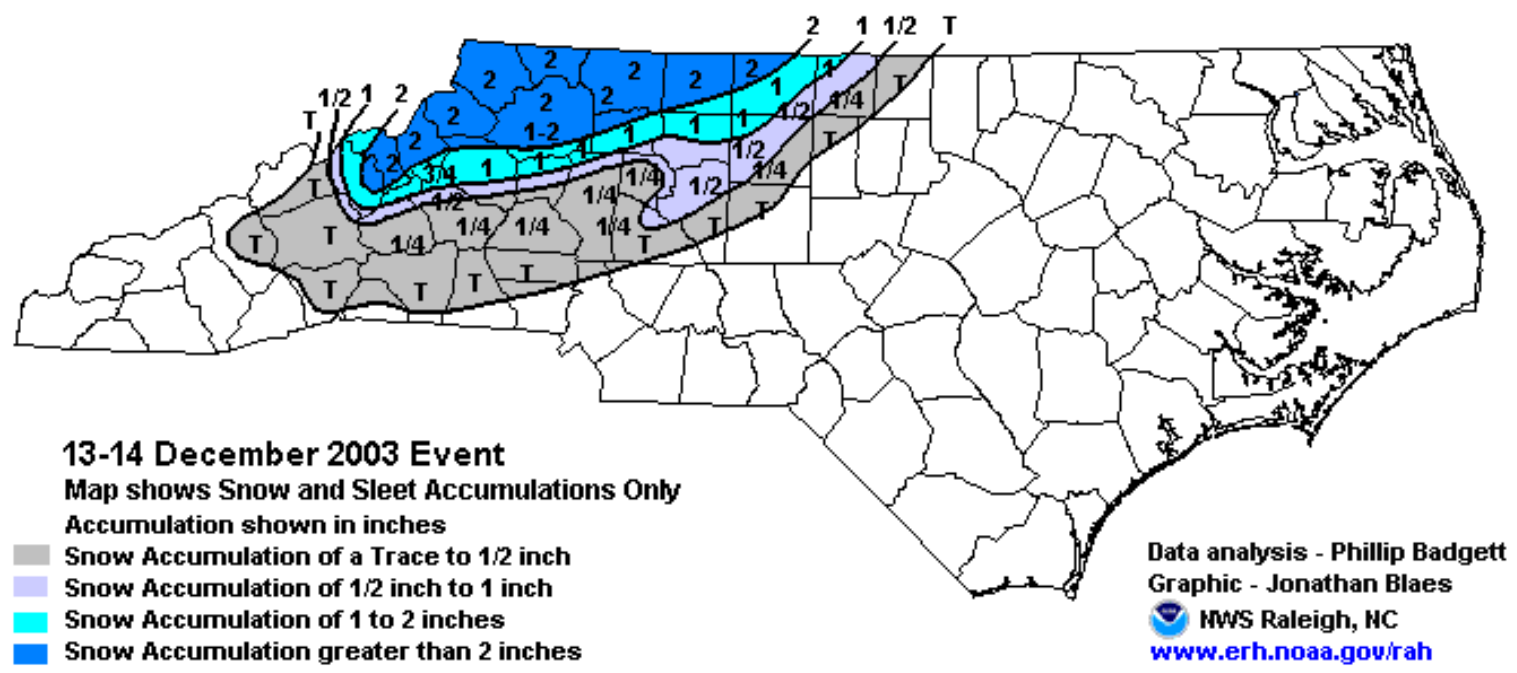

Figure A13. 13-14 December 2003 


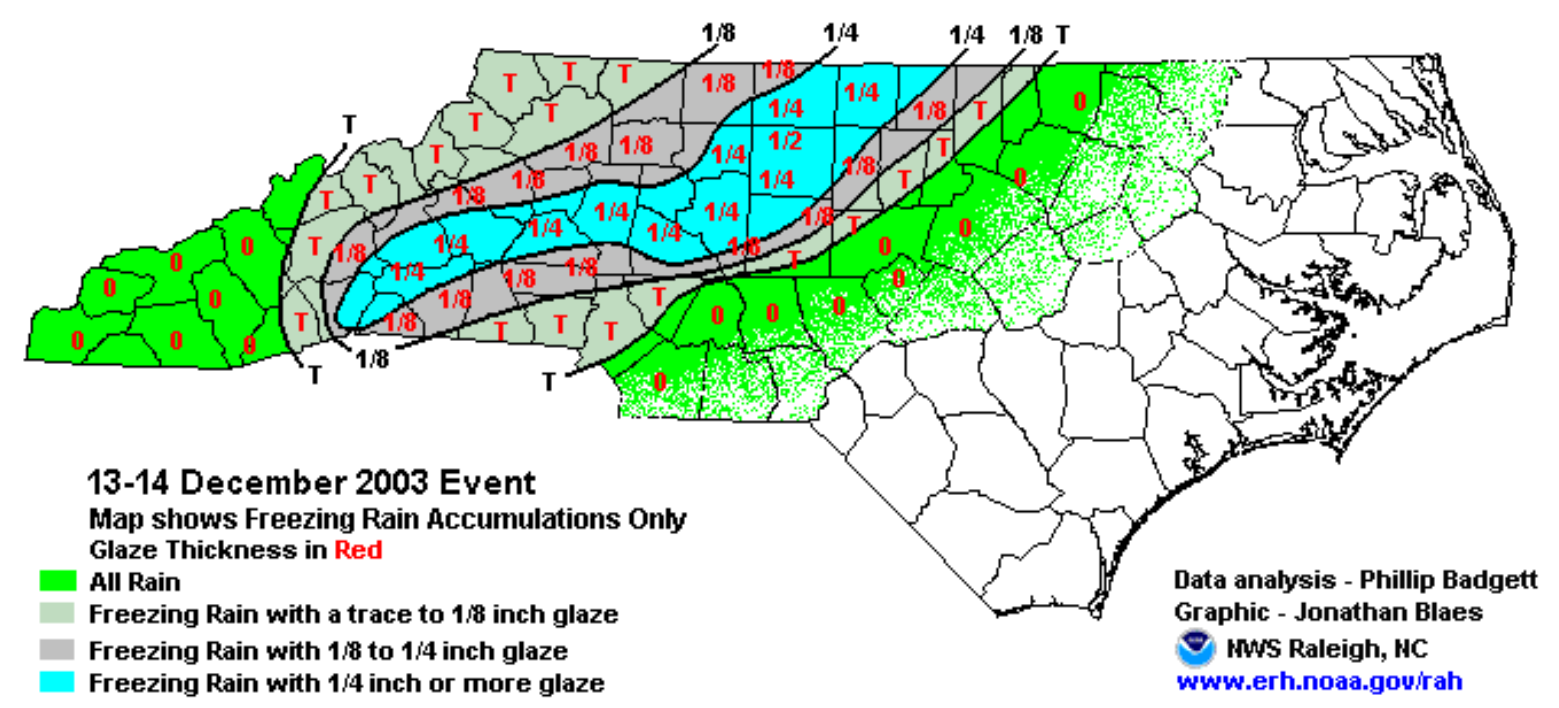

Figure A13. Continued

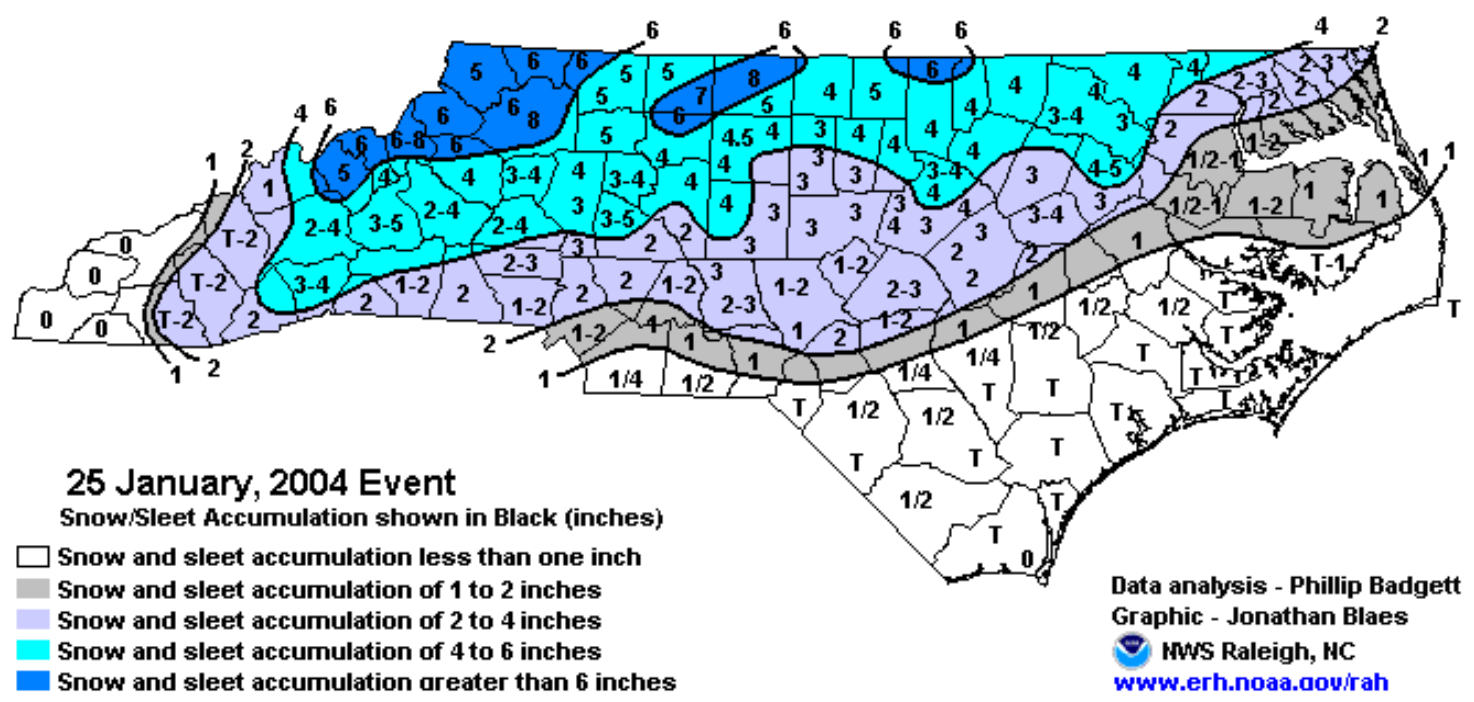

Figure A14. 25-26 January 2004 

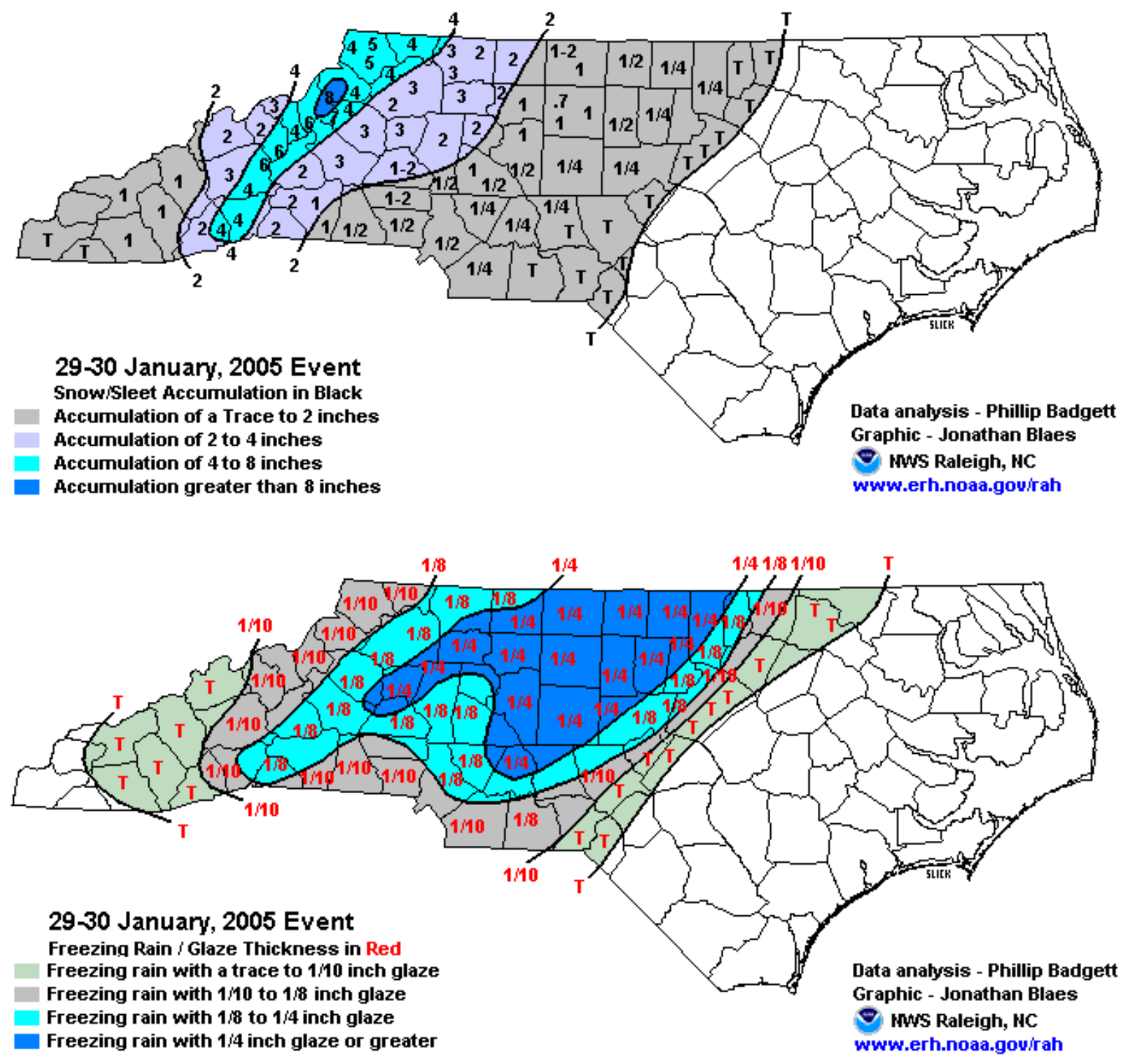

Figure A15. 29-30 January 2005 


\section{APPENDIX II}

Comparison of Observed and Modeled (HYSPLIT) Soundings

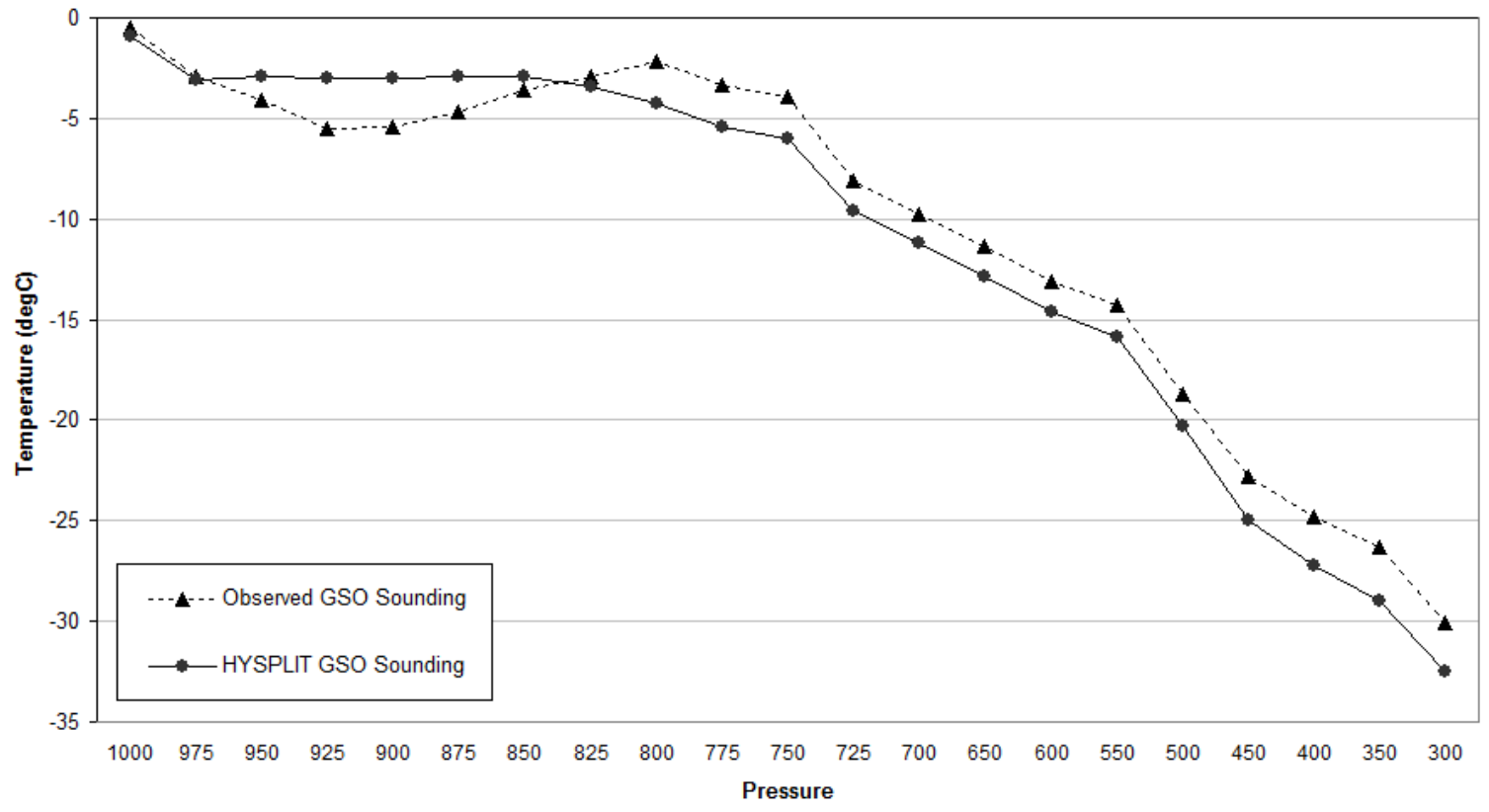

Figure A16. 06 UTC 25 January 2000

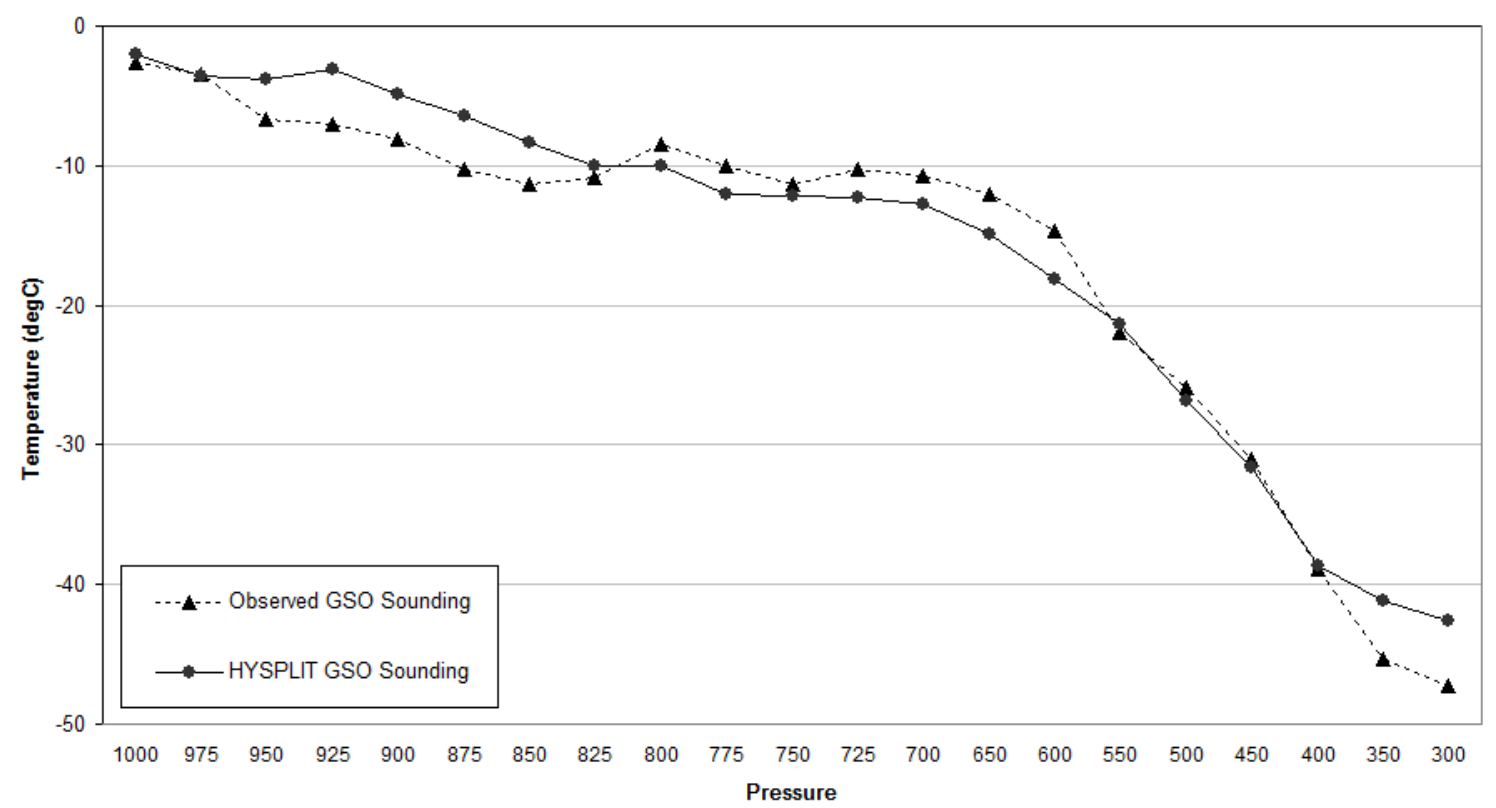

Figure A17. 12 UTC 2 March 2009 


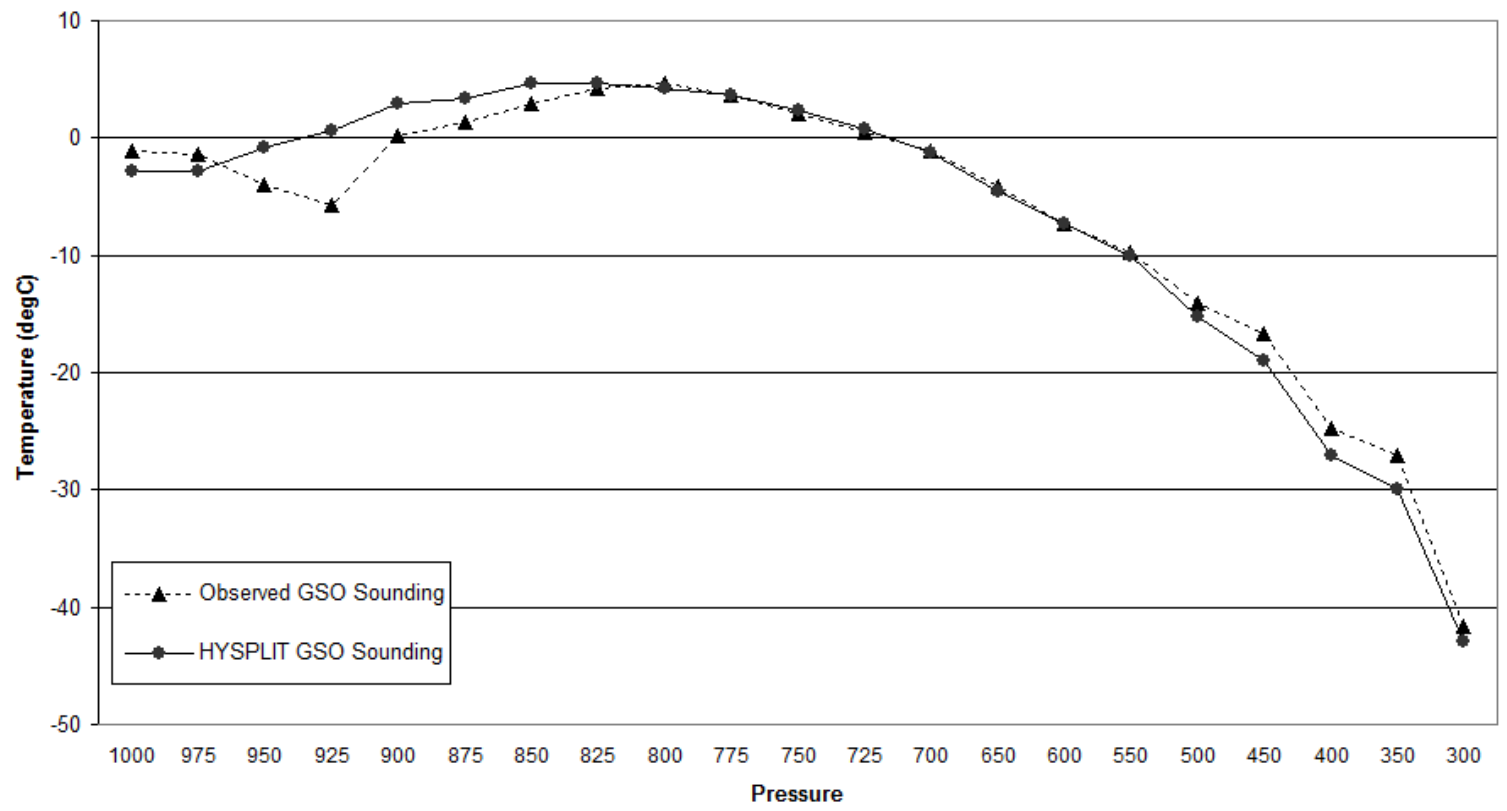

Figure A18. 06 UTC 5 December 2002

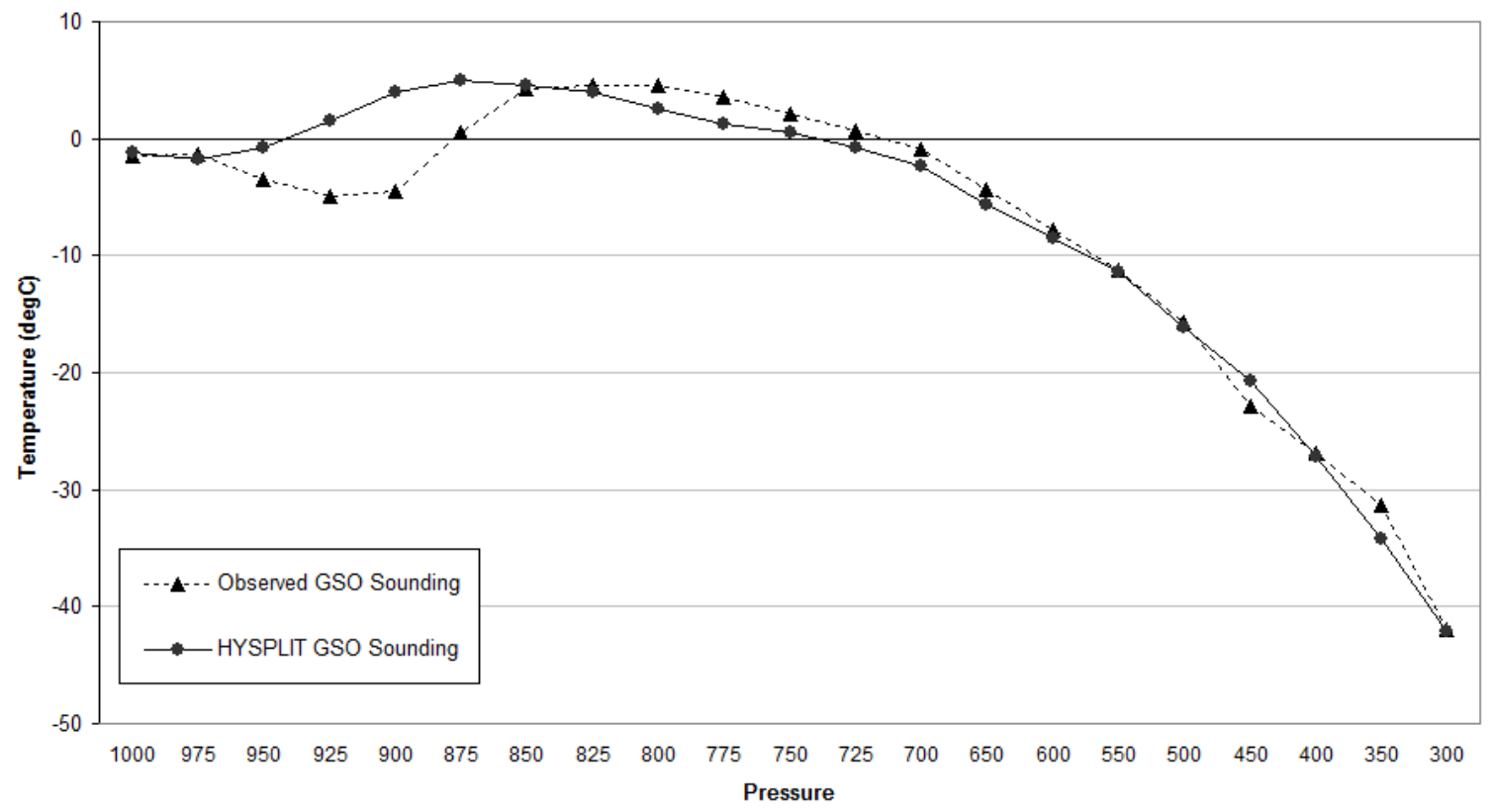

Figure A19. 06 UTC 14 December 2003 


\section{APPENDIX III}

Composite Trajectories with Range Bars

Chapter 3: Snowstorms

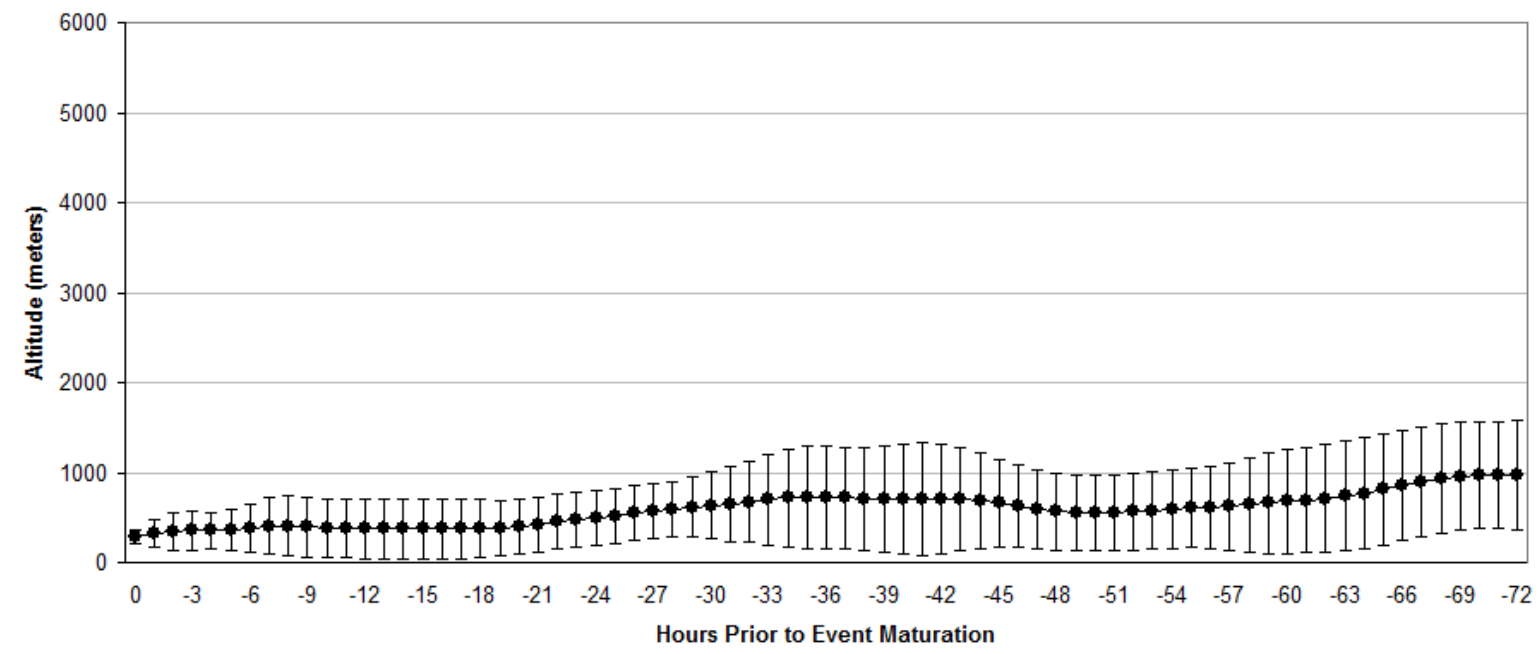

Figure A20. Vertical trajectory composite with range bars of air parcels terminating at the near-surface level in the region of heavy snowfall.

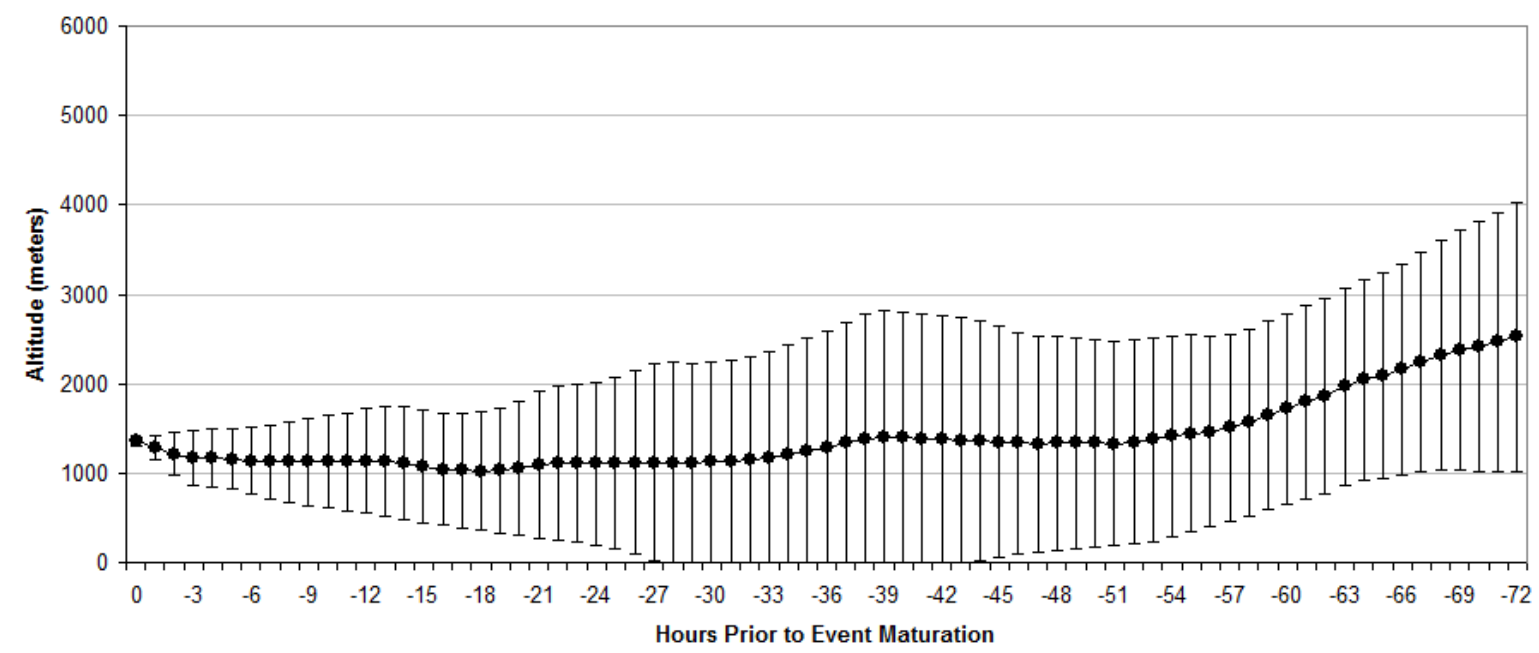

Figure A21. Vertical trajectory composite with range bars of air parcels terminating in the warm layer zone. 


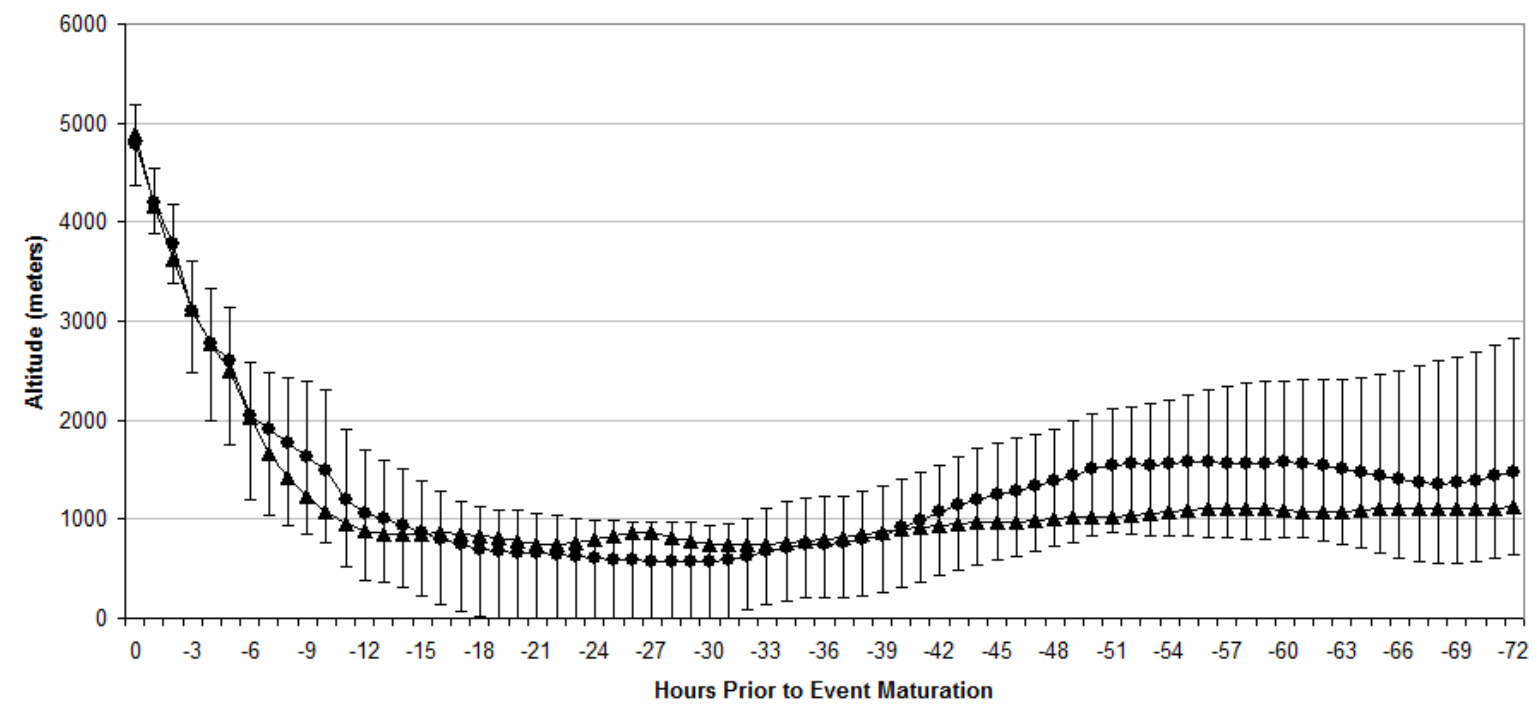

Figure A22. Vertical trajectory composite with range bars of air parcels terminating in the dendritic growth zone (DGZ). Circles represent the DGZ-anticyclonic trajectory and triangles represent the DGZ-cyclonic trajectory.

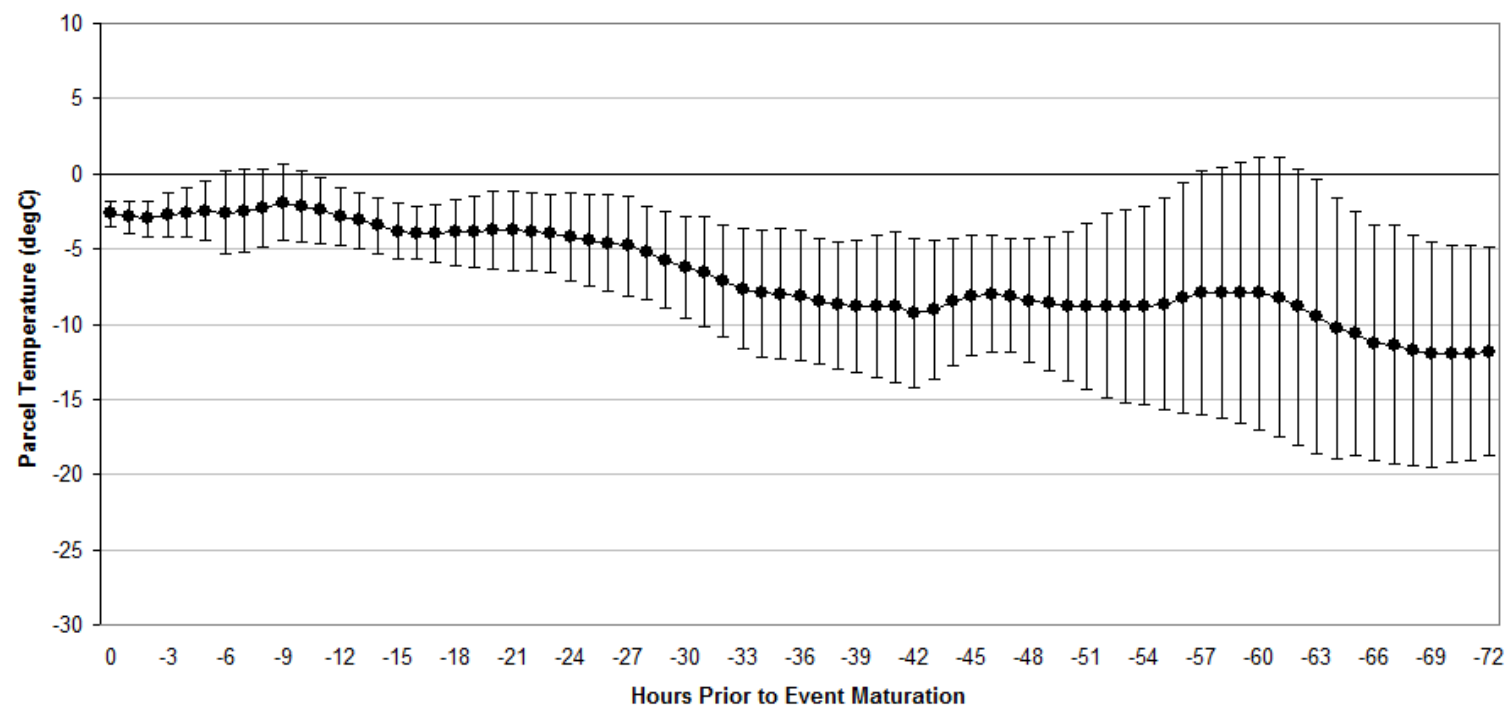

Figure A23. Composite near-surface temperature trajectory with range bars. 


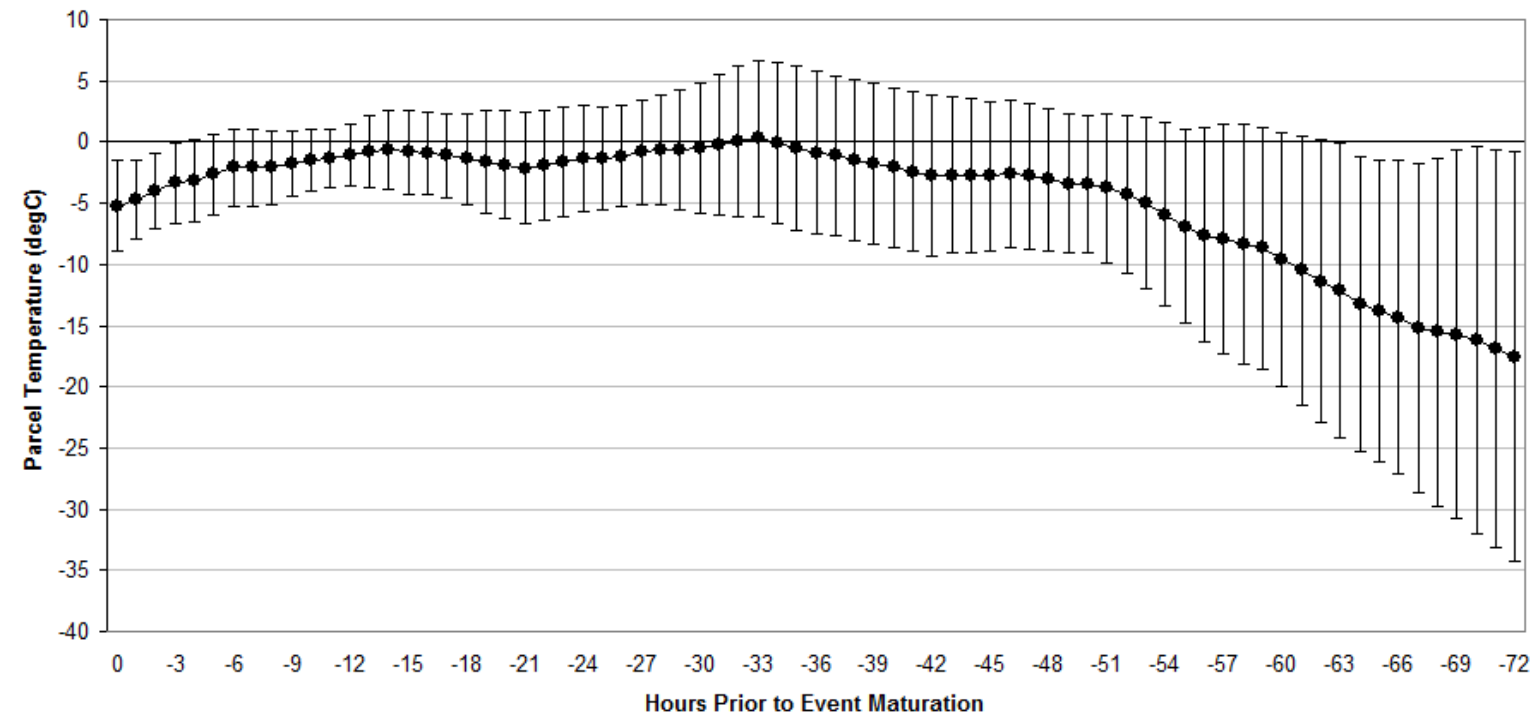

Figure A24. Composite warm layer zone temperature trajectory with range bars.

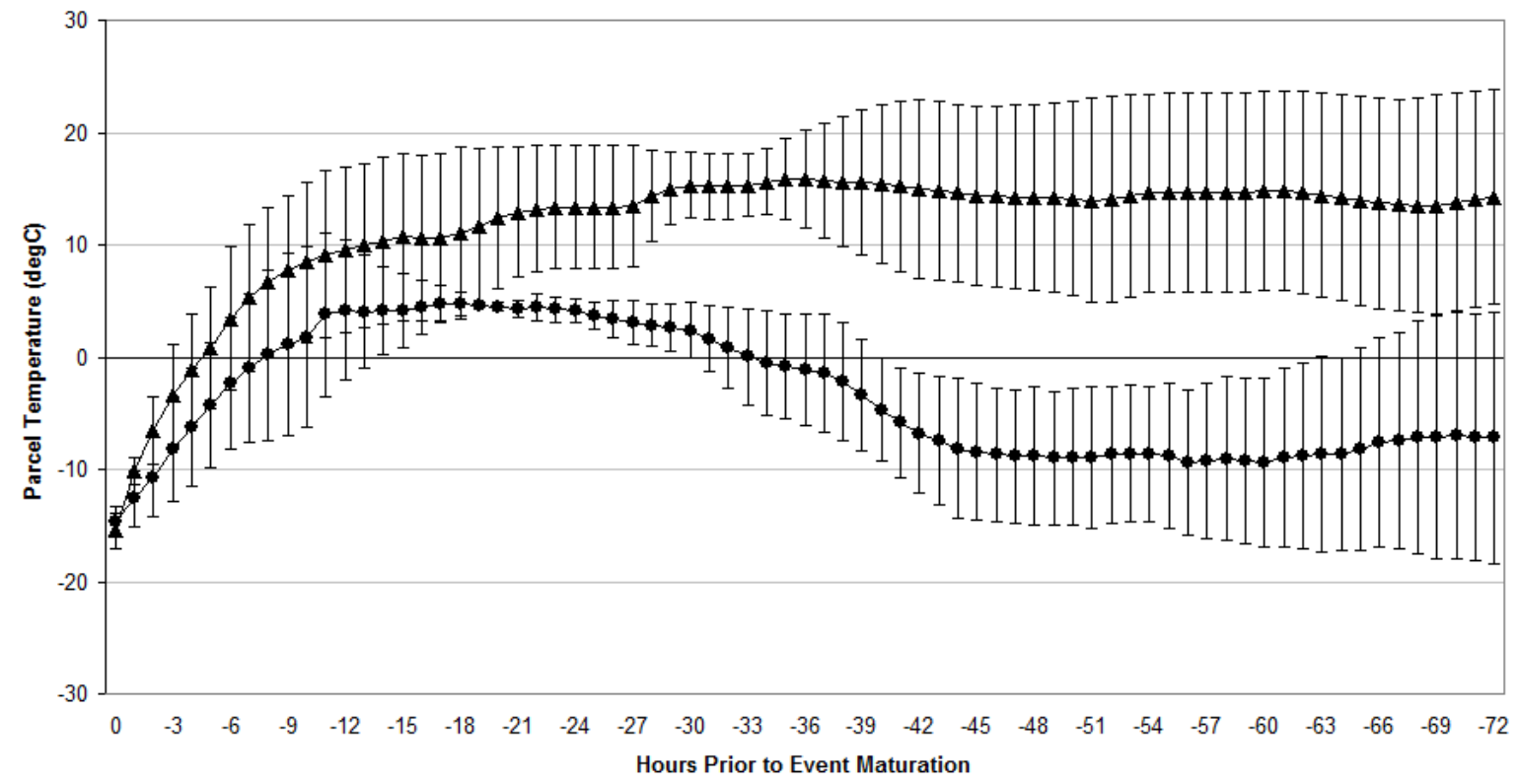

Figure A25. Composite DGZ temperature trajectories with range bars. Circles represent the DGZ-anticyclonic trajectory and triangles represent the DGZ-cyclonic trajectory. 


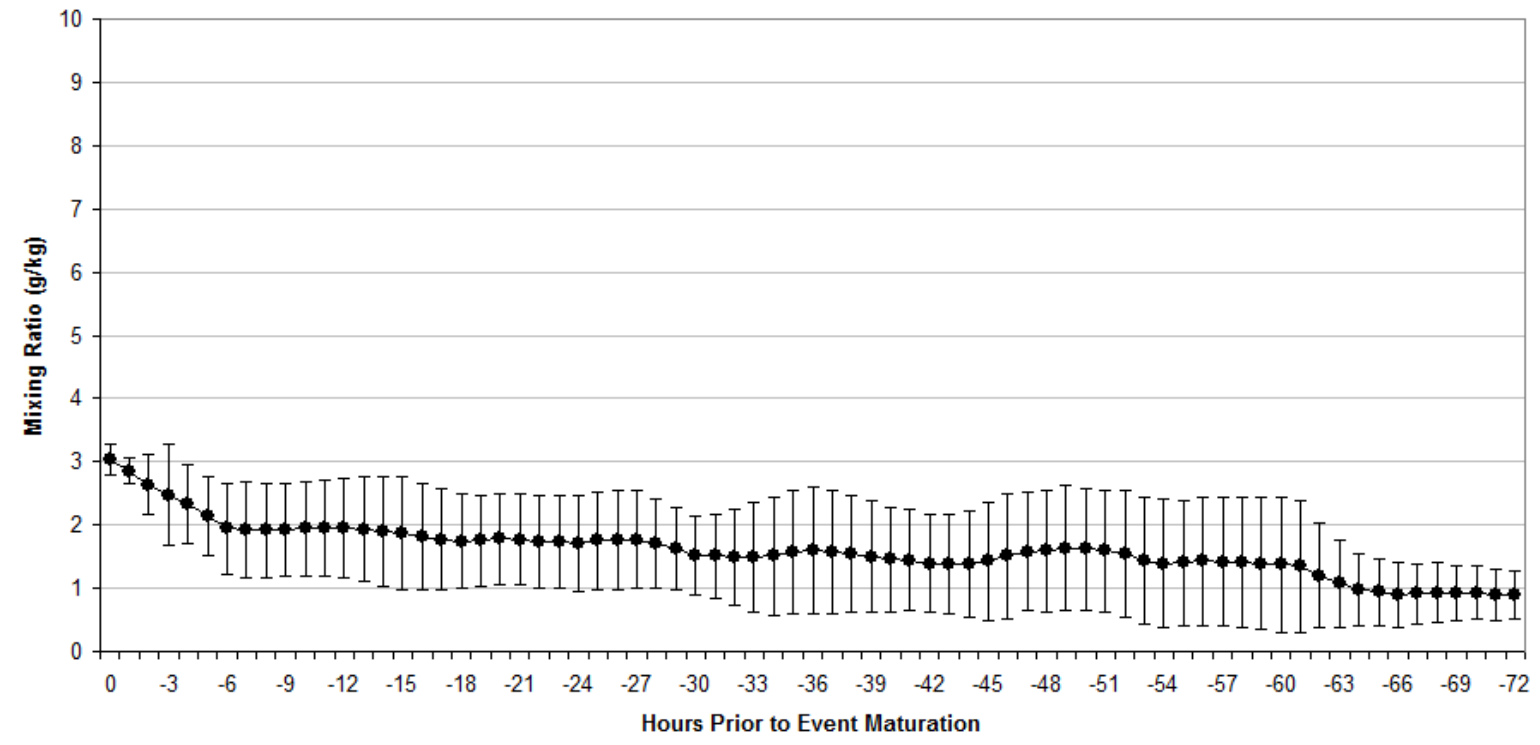

Figure A26. Moisture composite with range bars along the near-surface trajectory.

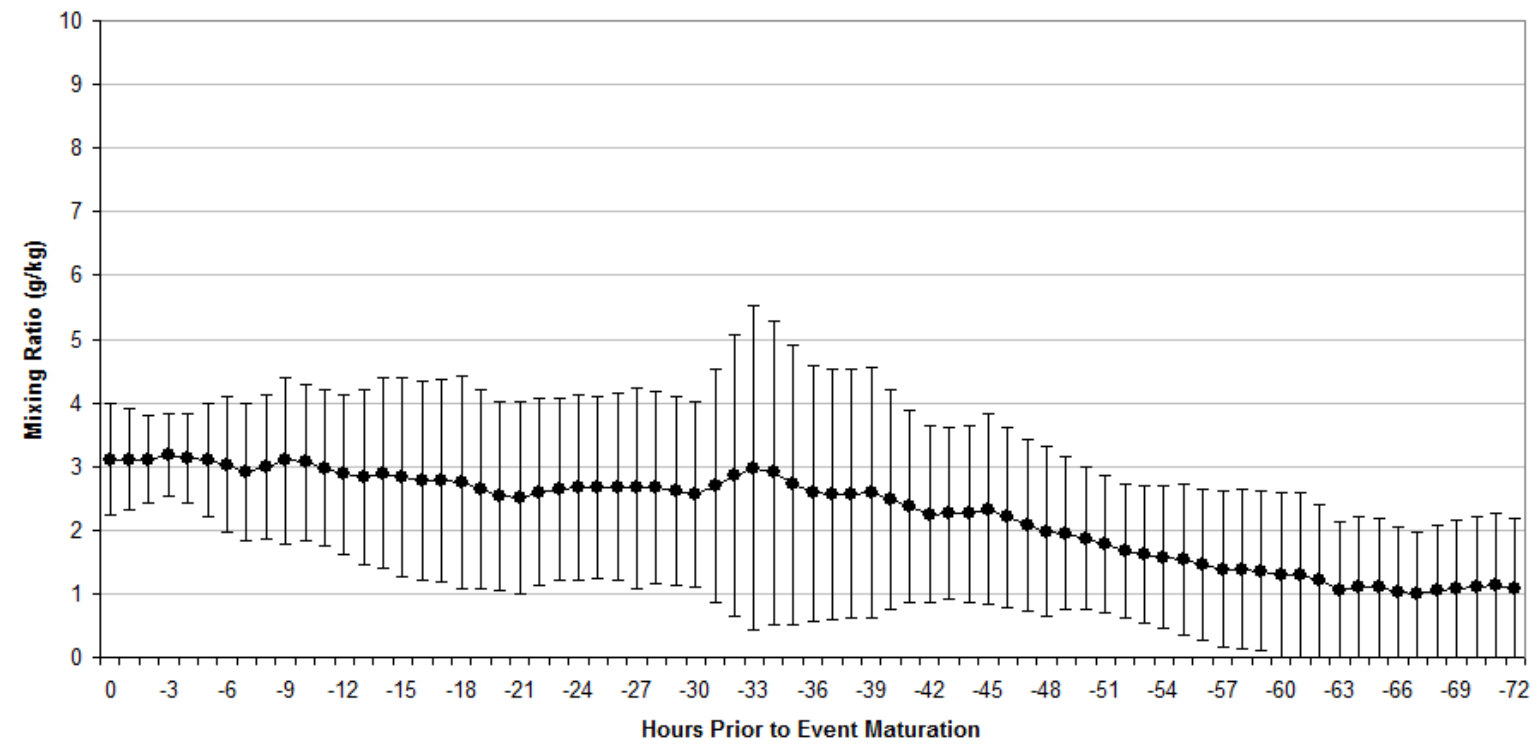

Figure A27. Moisture composite with range bars along the warm layer zone trajectory. 


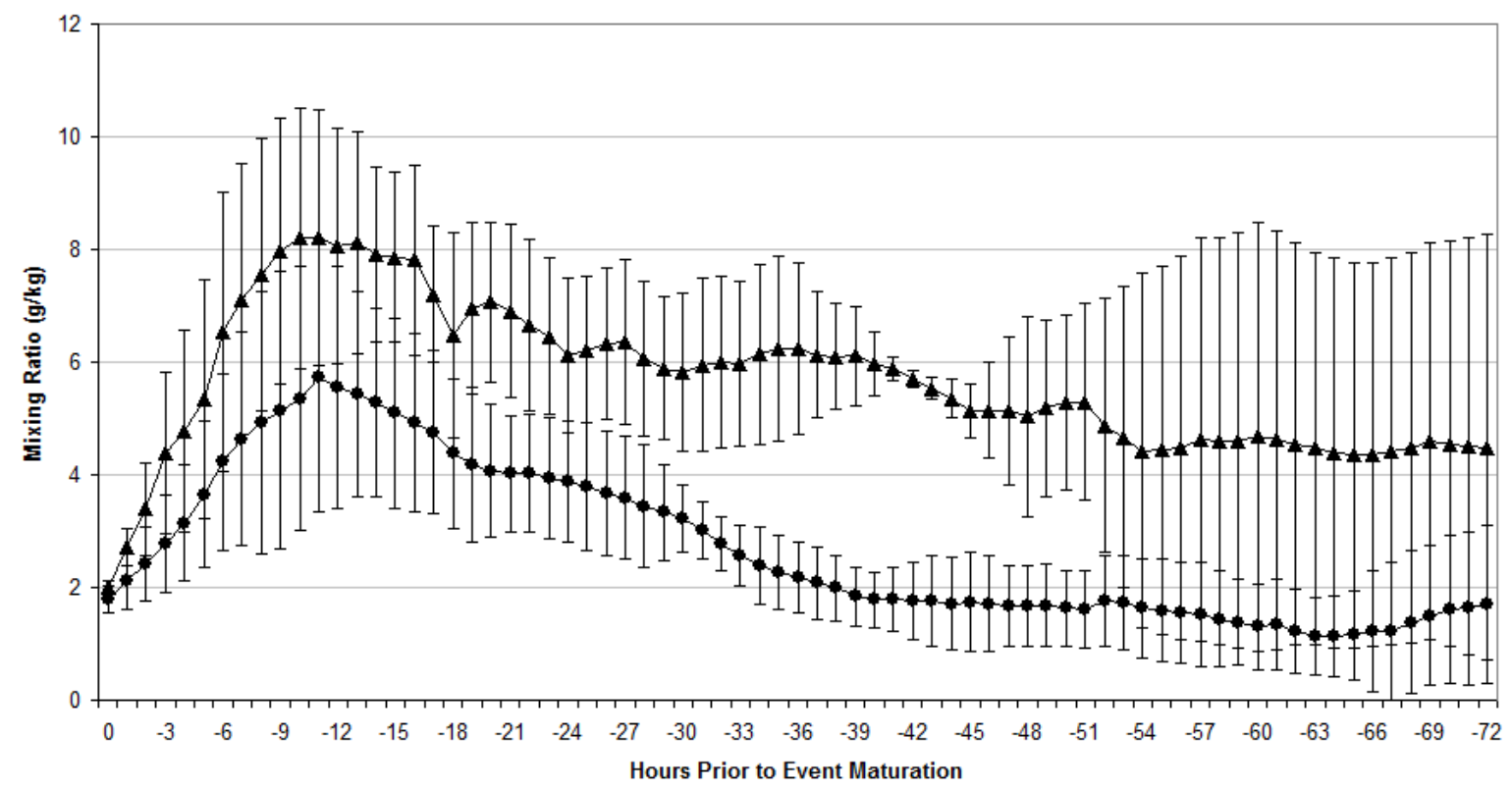

Figure A28. Moisture composites with range bars along the DGZ trajectories. Circles represent the DGZ-anticyclonic trajectory and triangles represent the DGZ-cyclonic trajectory.

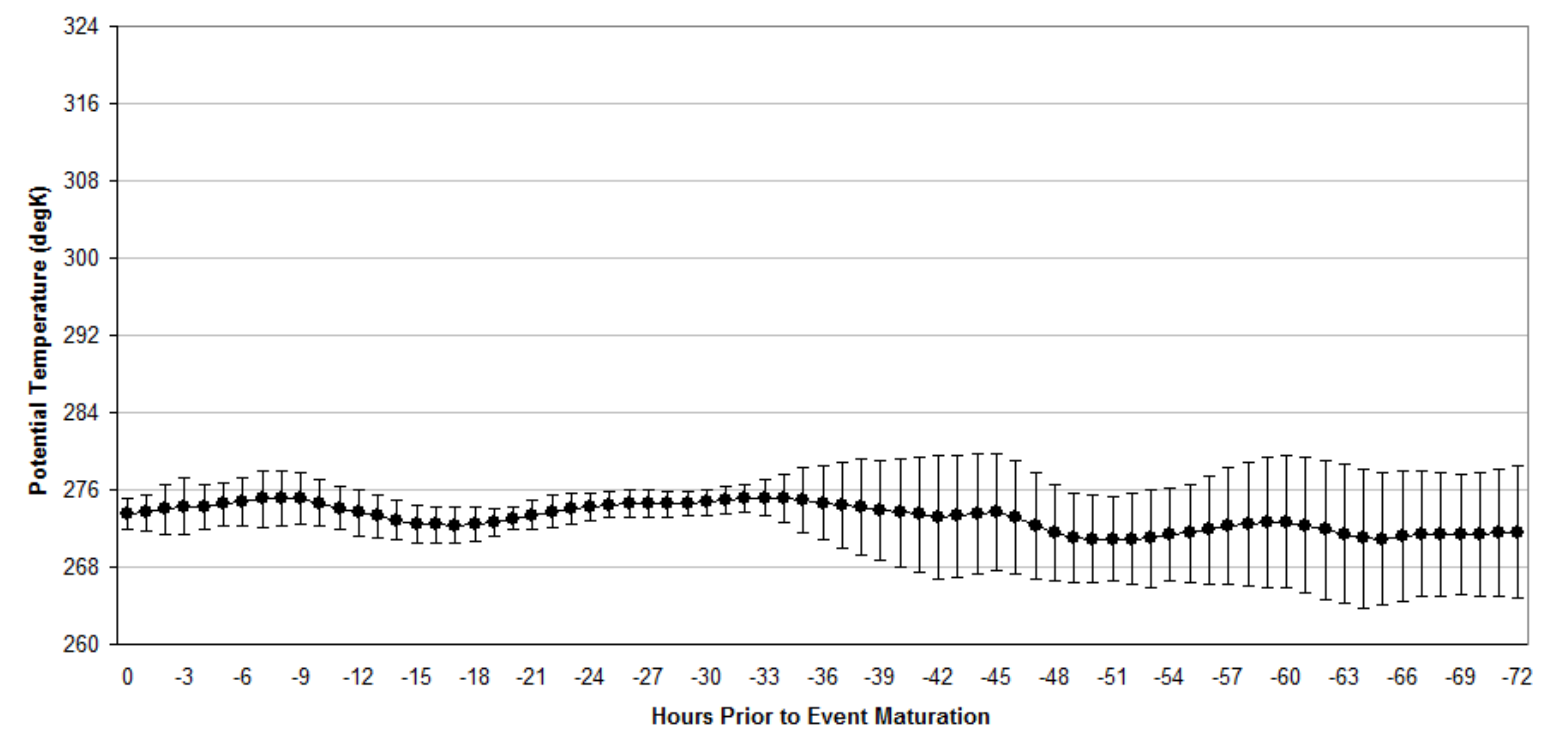

Figure A29. Composite near-surface trajectory of potential temperature with range bars. 


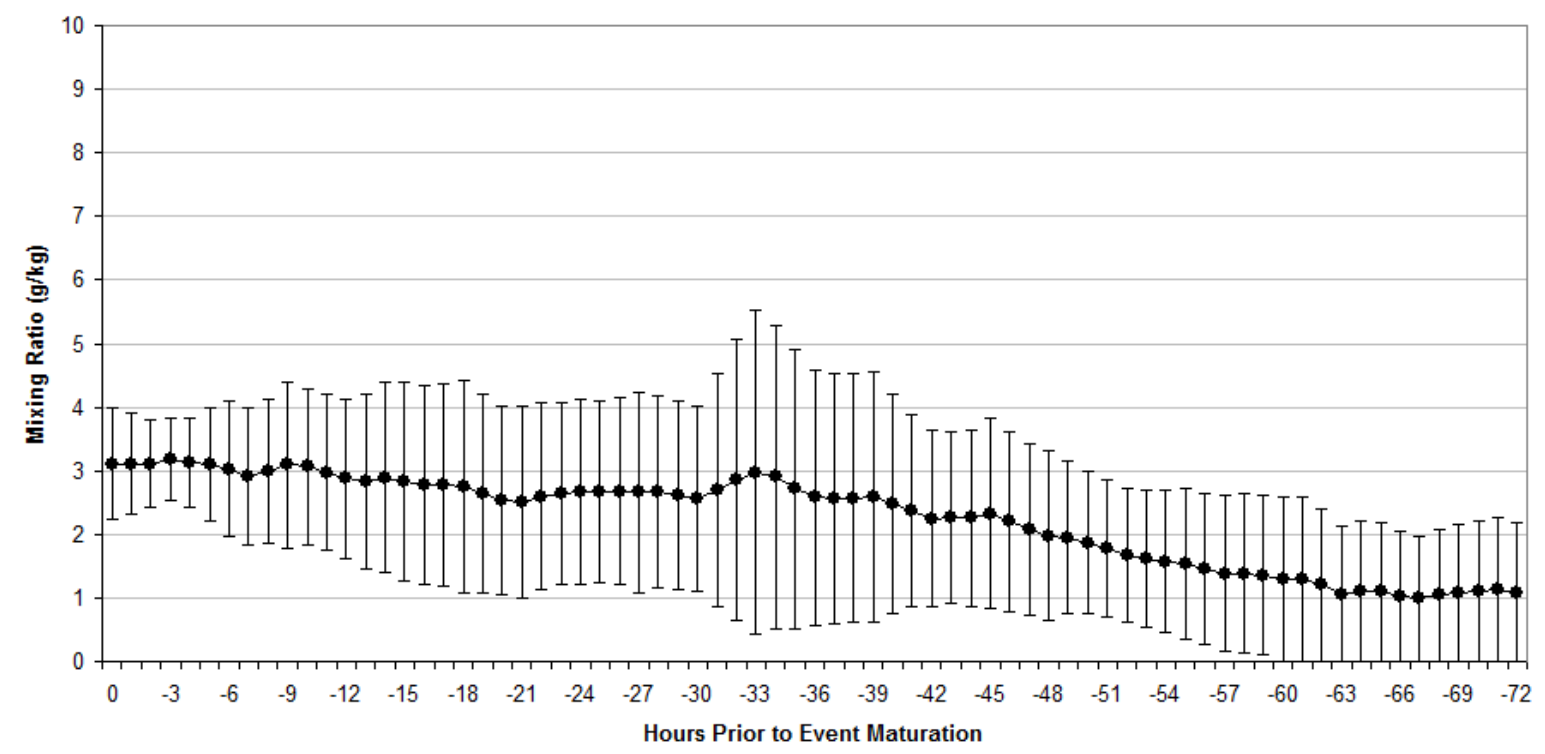

Figure A30. Composite warm layer zone trajectory of potential temperature with range bars.

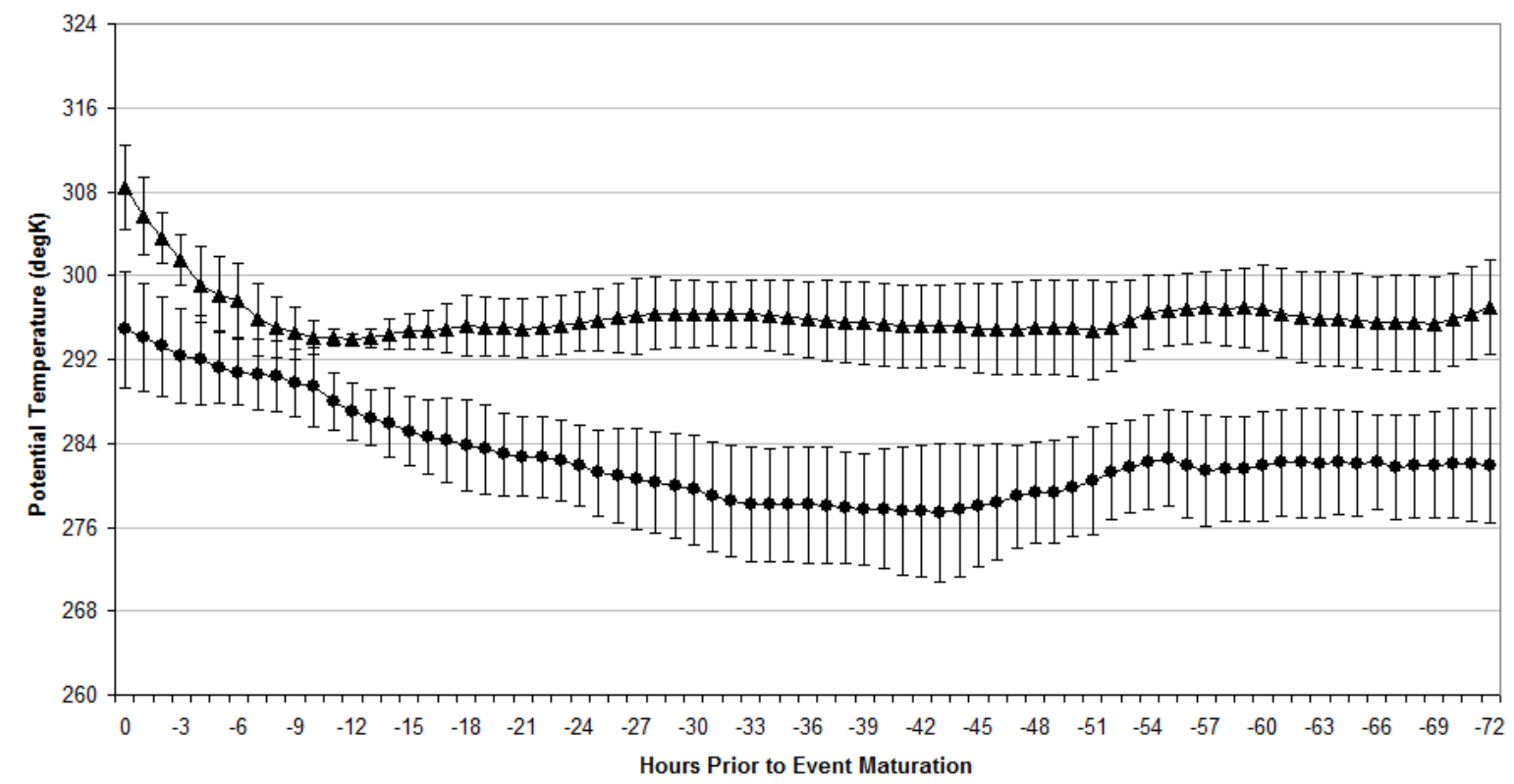

Figure A31. Composite DGZ trajectories of potential temperature with range bars. Circles represent the DGZ-anticyclonic trajectory and triangles represent the DGZ-cyclonic trajectory. 


\section{Chapter 4: Ice Storms}

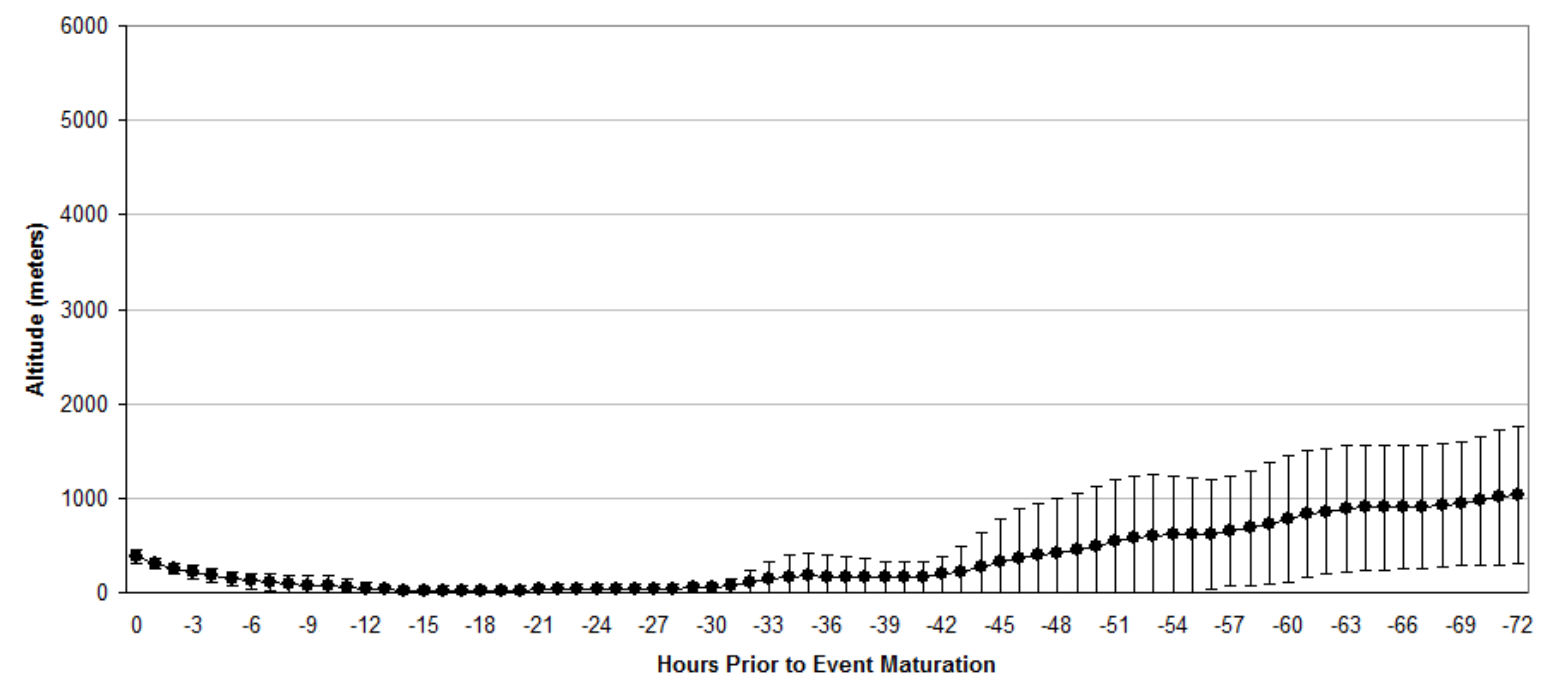

Figure A32. Vertical trajectory composite with range bars of air parcels terminating at the near-surface level in the region of heavy freezing precipitation.

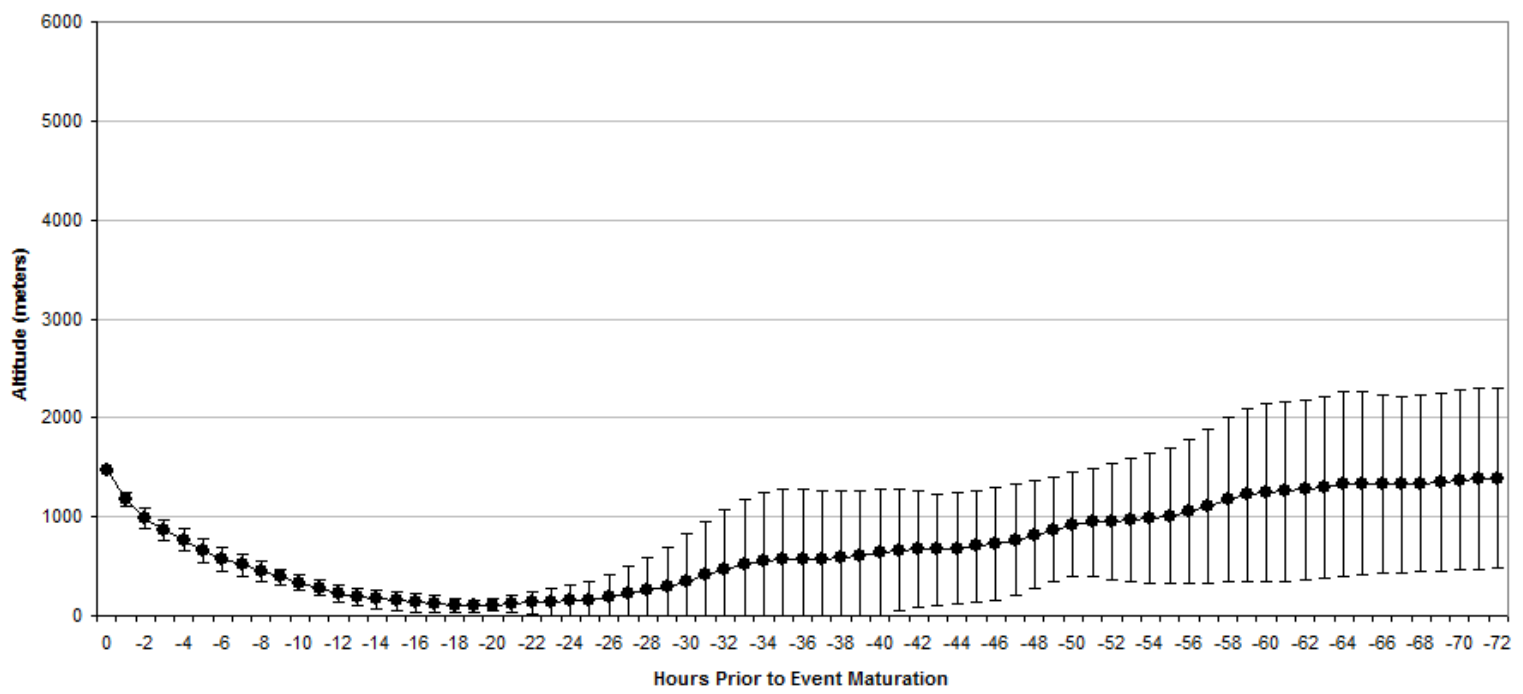

Figure A33. Vertical trajectory composite with range bars of air parcels terminating in the warm layer zone. 


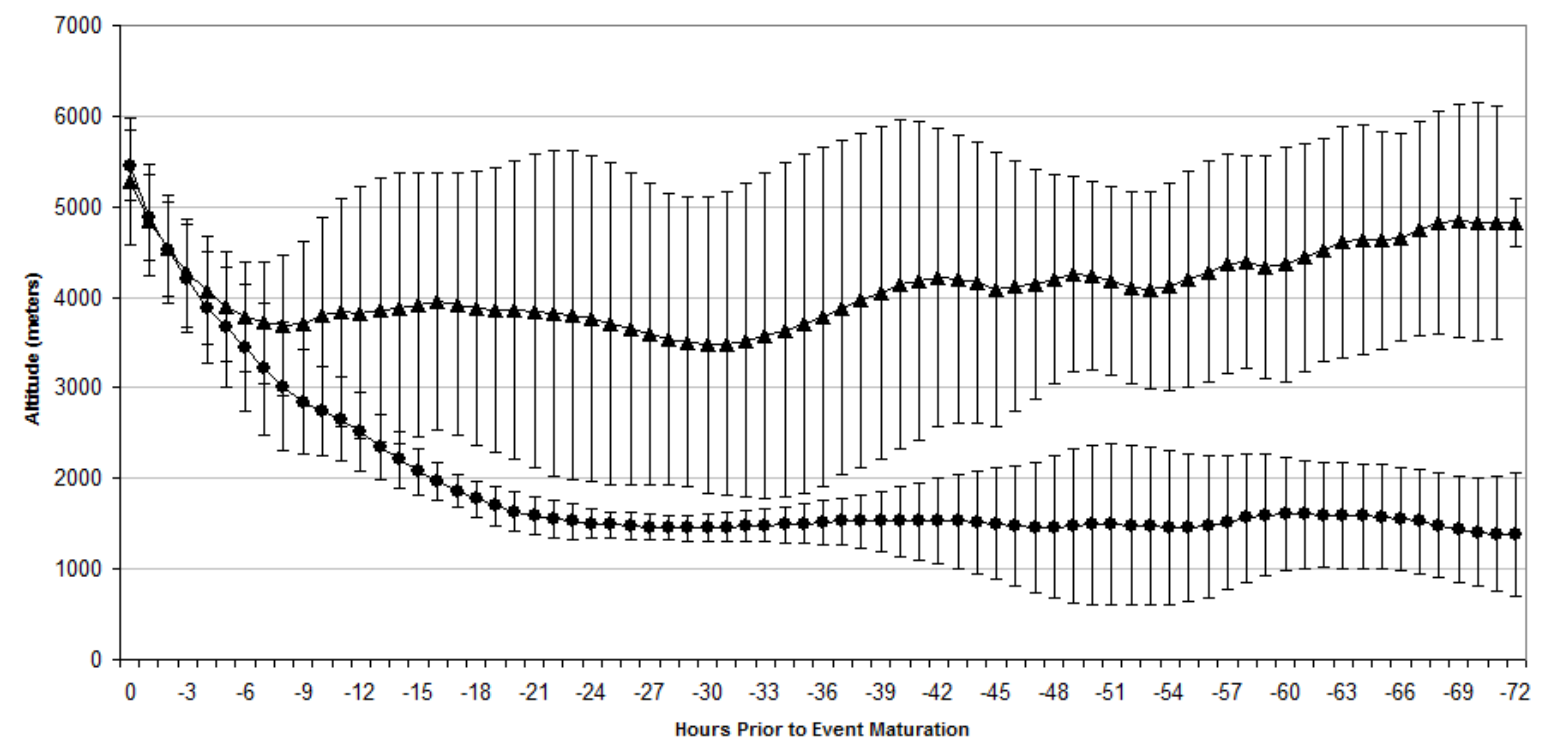

Figure A34. Vertical trajectory composite with range bars of air parcels terminating in the dendritic growth zone (DGZ). Circles represent the DGZ-anticyclonic trajectory and triangles represent the DGZ-cyclonic trajectory.

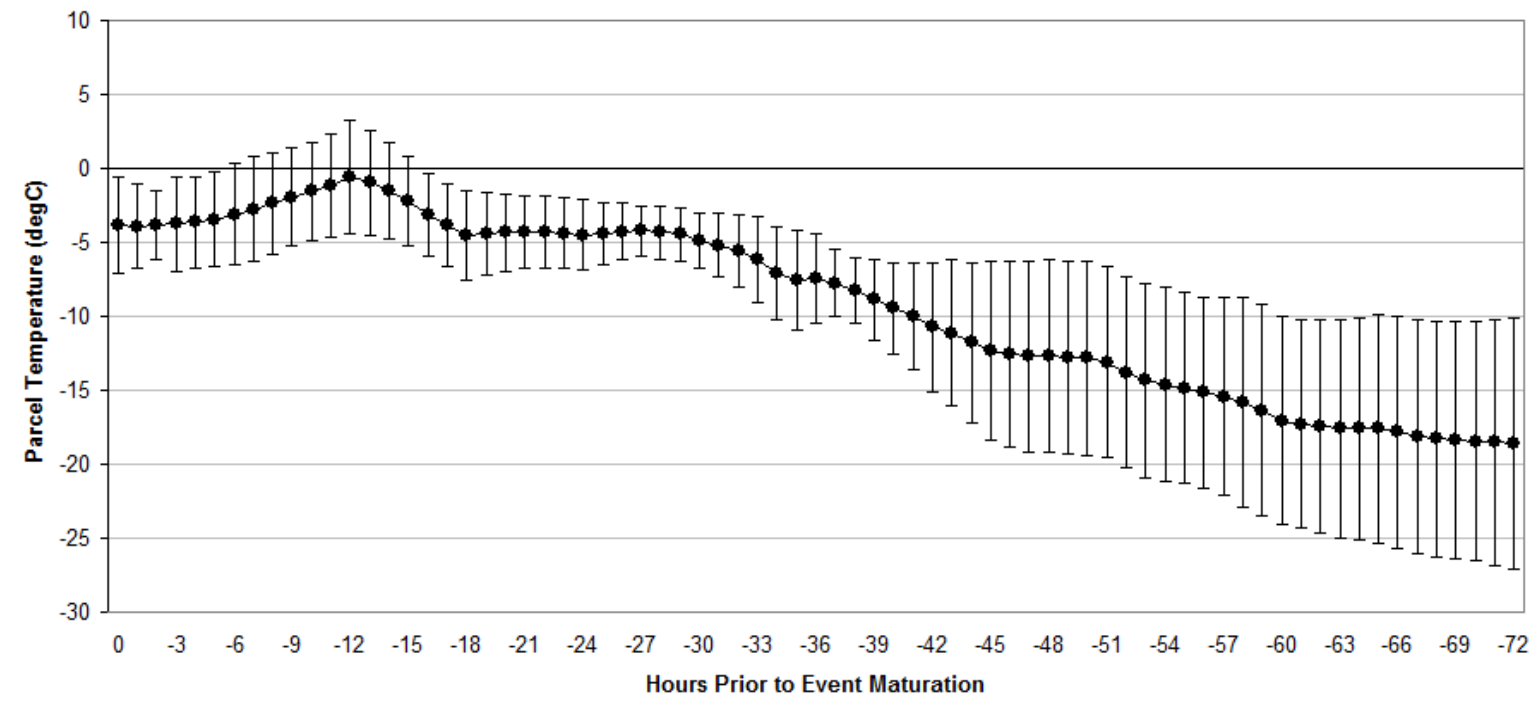

Figure A35. Figure A23. Composite near-surface temperature trajectory with range bars. 


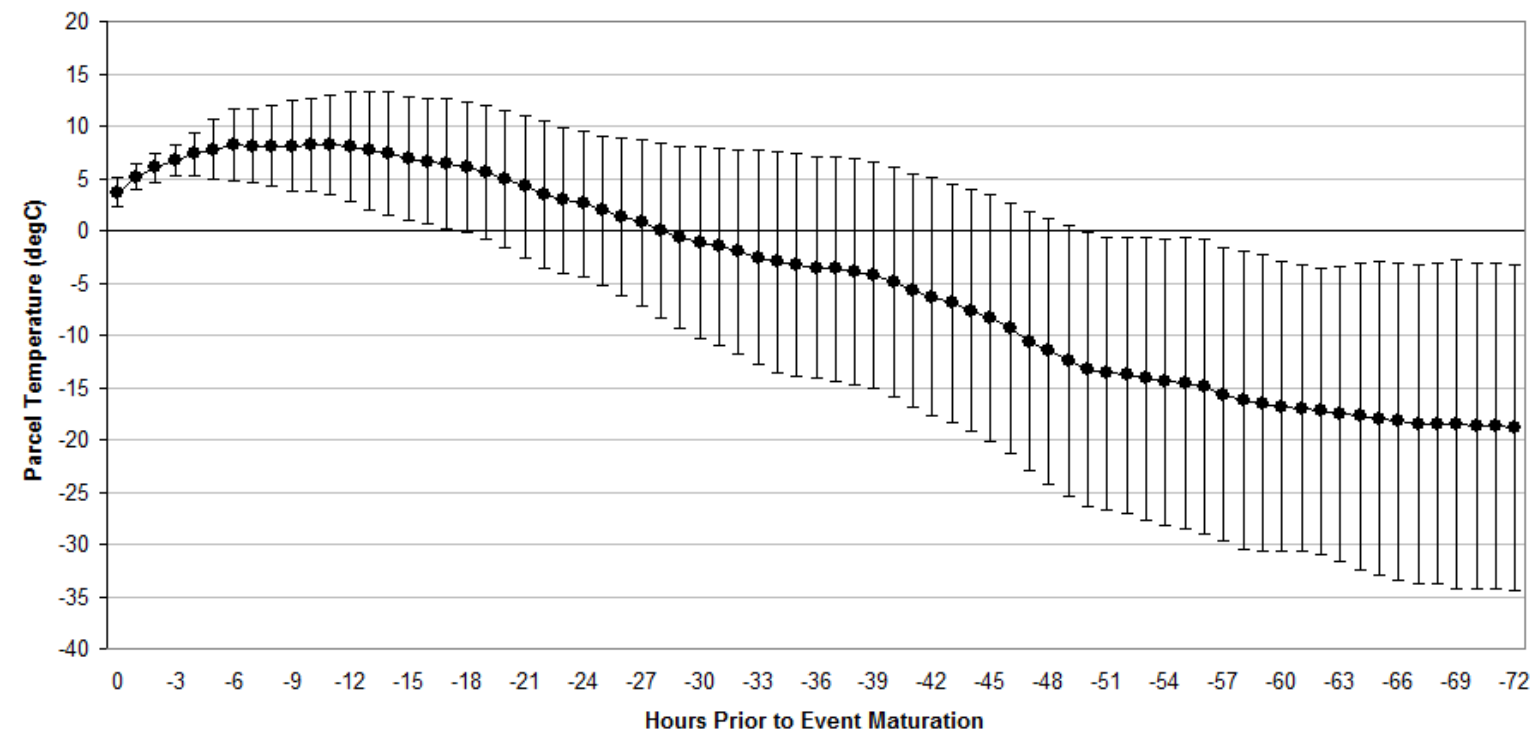

Figure A36. Composite warm layer zone temperature trajectory with range bars.

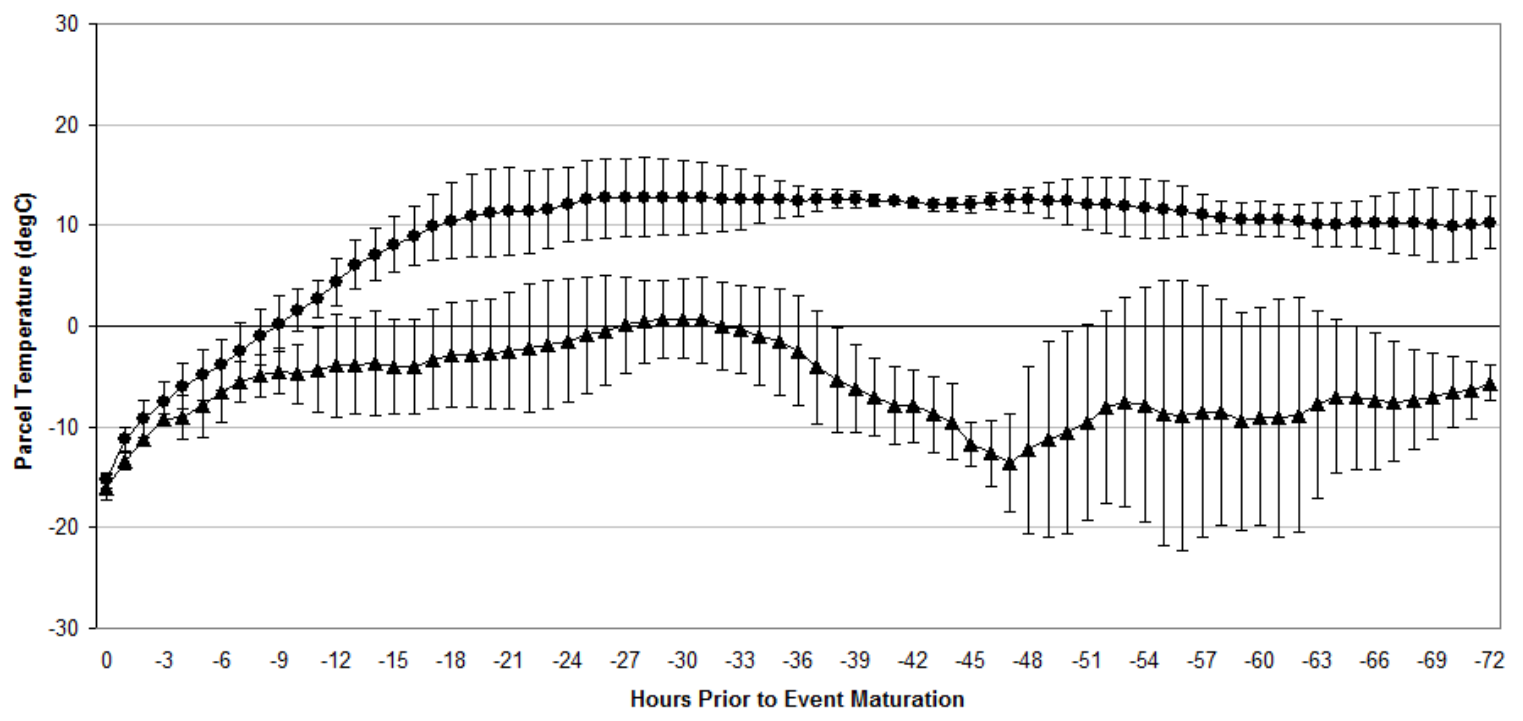

Figure A37. Composite DGZ temperature trajectories with range bars. Circles represent the DGZ-anticyclonic trajectory and triangles represent the DGZ-cyclonic trajectory. 


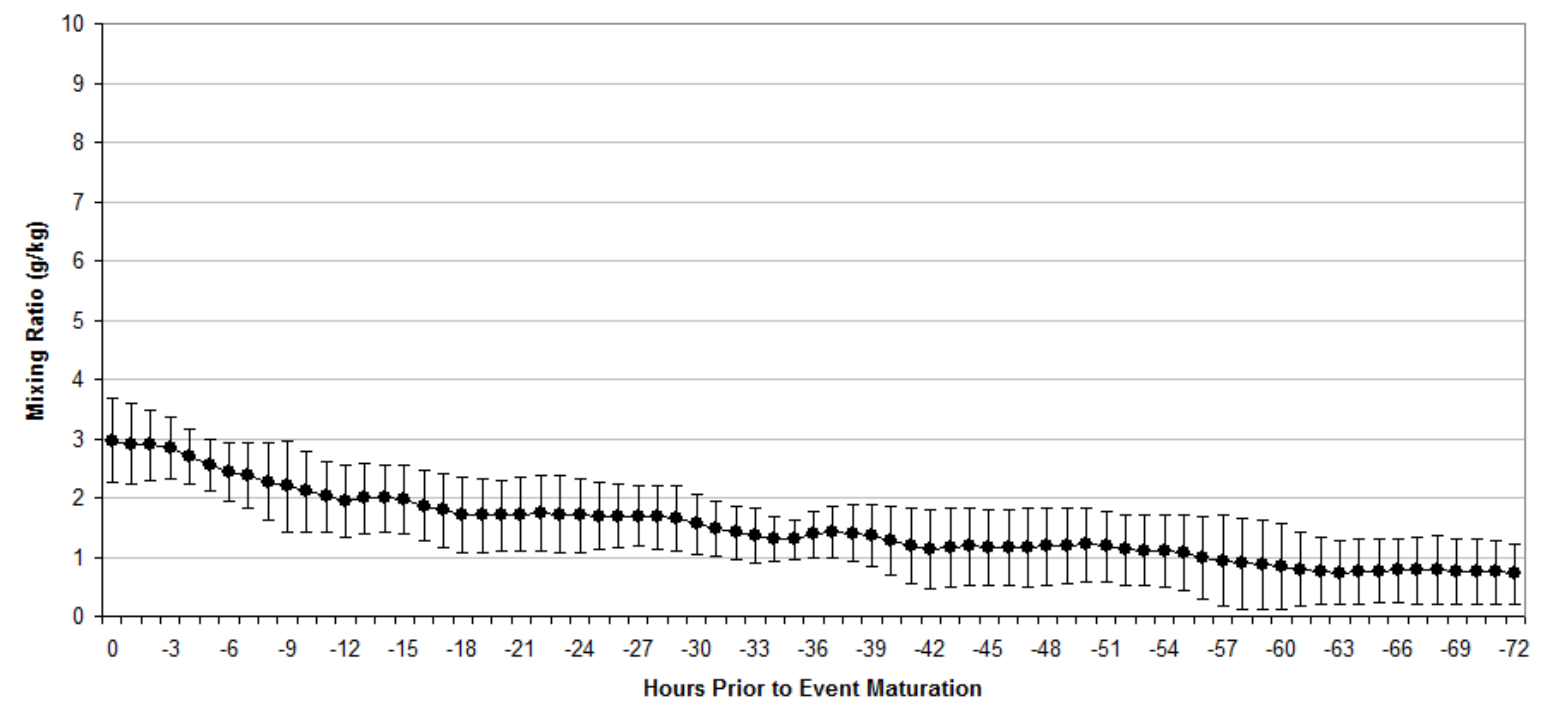

Figure A38. Moisture composite with range bars along the near-surface trajectory.

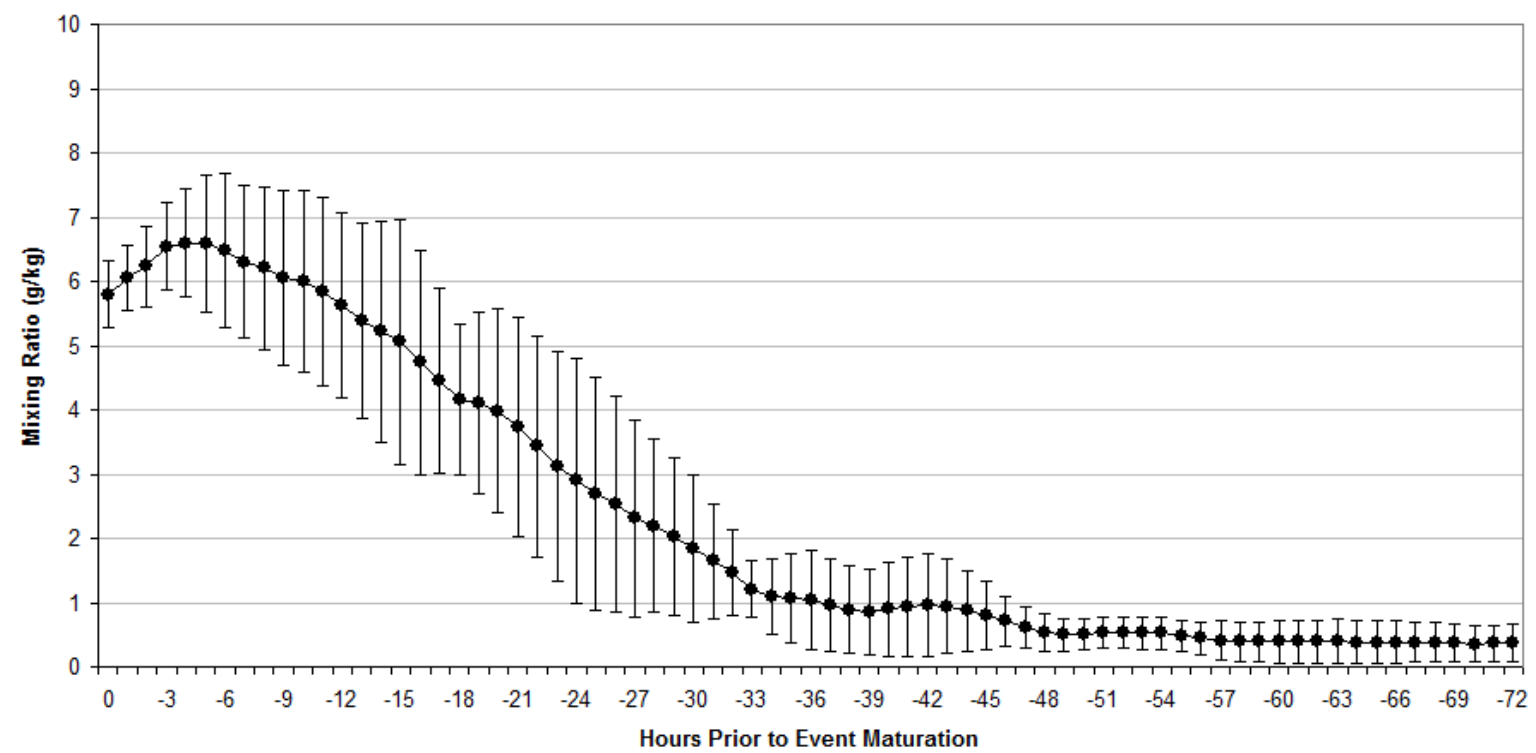

Figure A39. Moisture composite with range bars along the warm layer zone trajectory. 


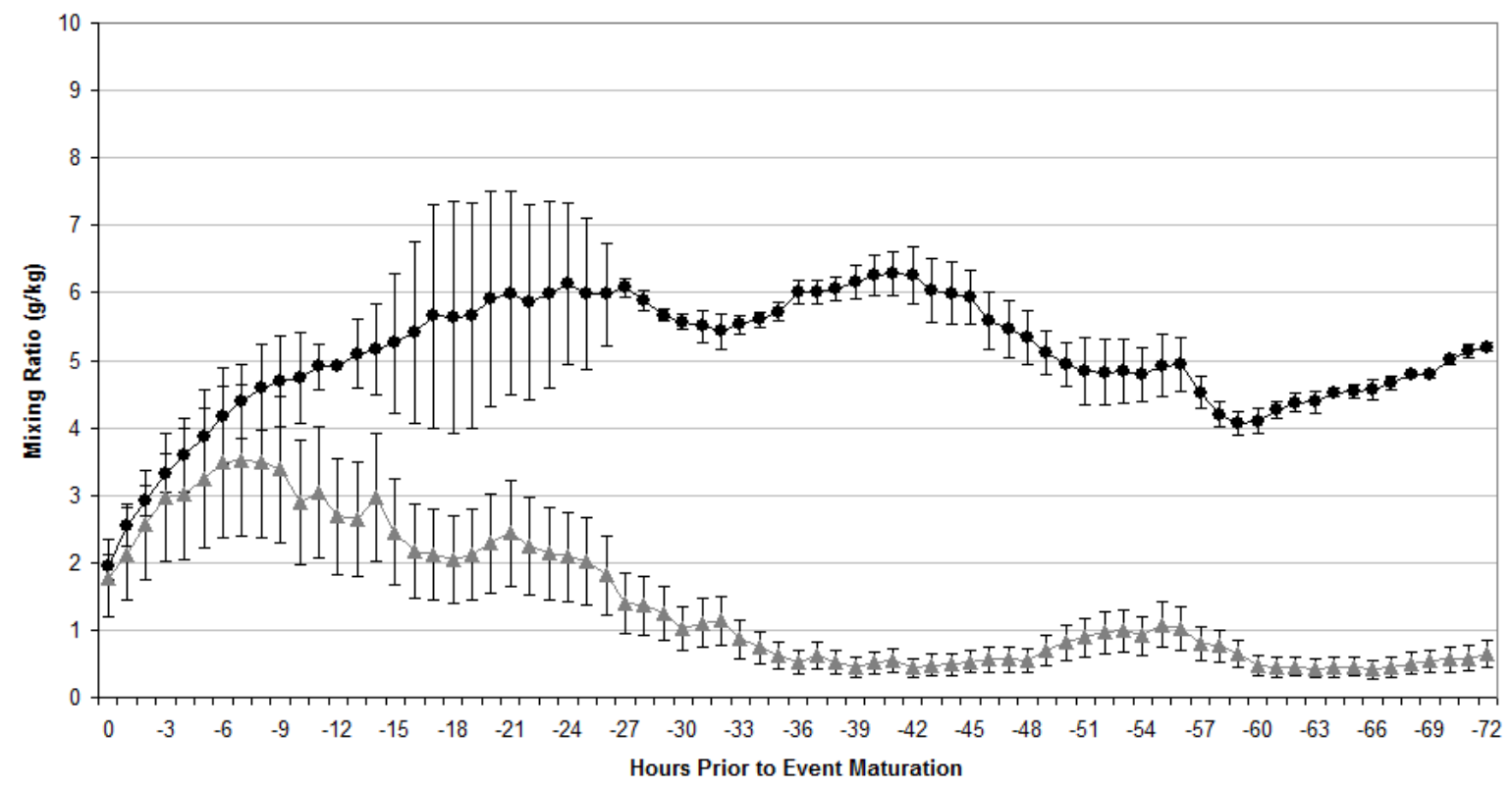

Figure A40. Moisture composites with range bars along the DGZ trajectories. Circles represent the DGZ-anticyclonic trajectory and triangles represent the DGZ-cyclonic trajectory.

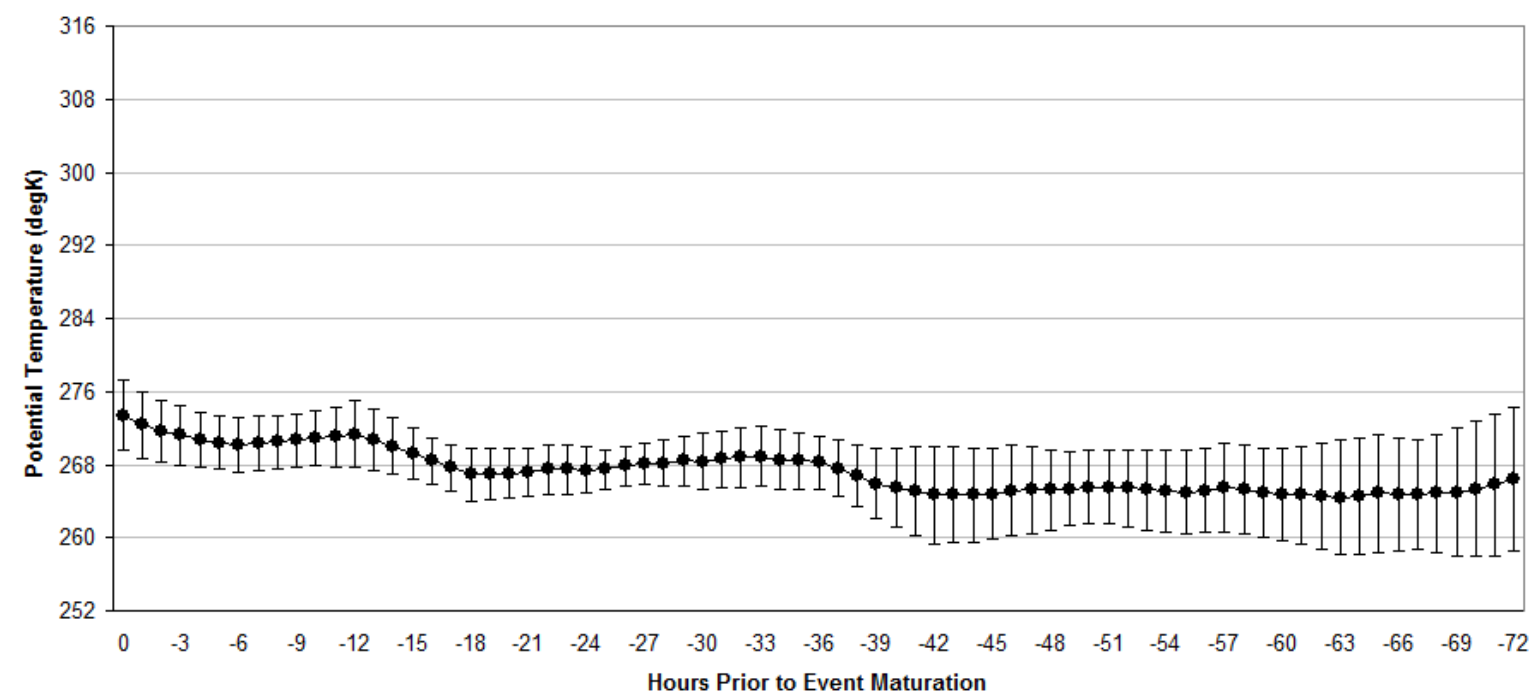

Figure A41. Composite near-surface trajectory of potential temperature with range bars. 


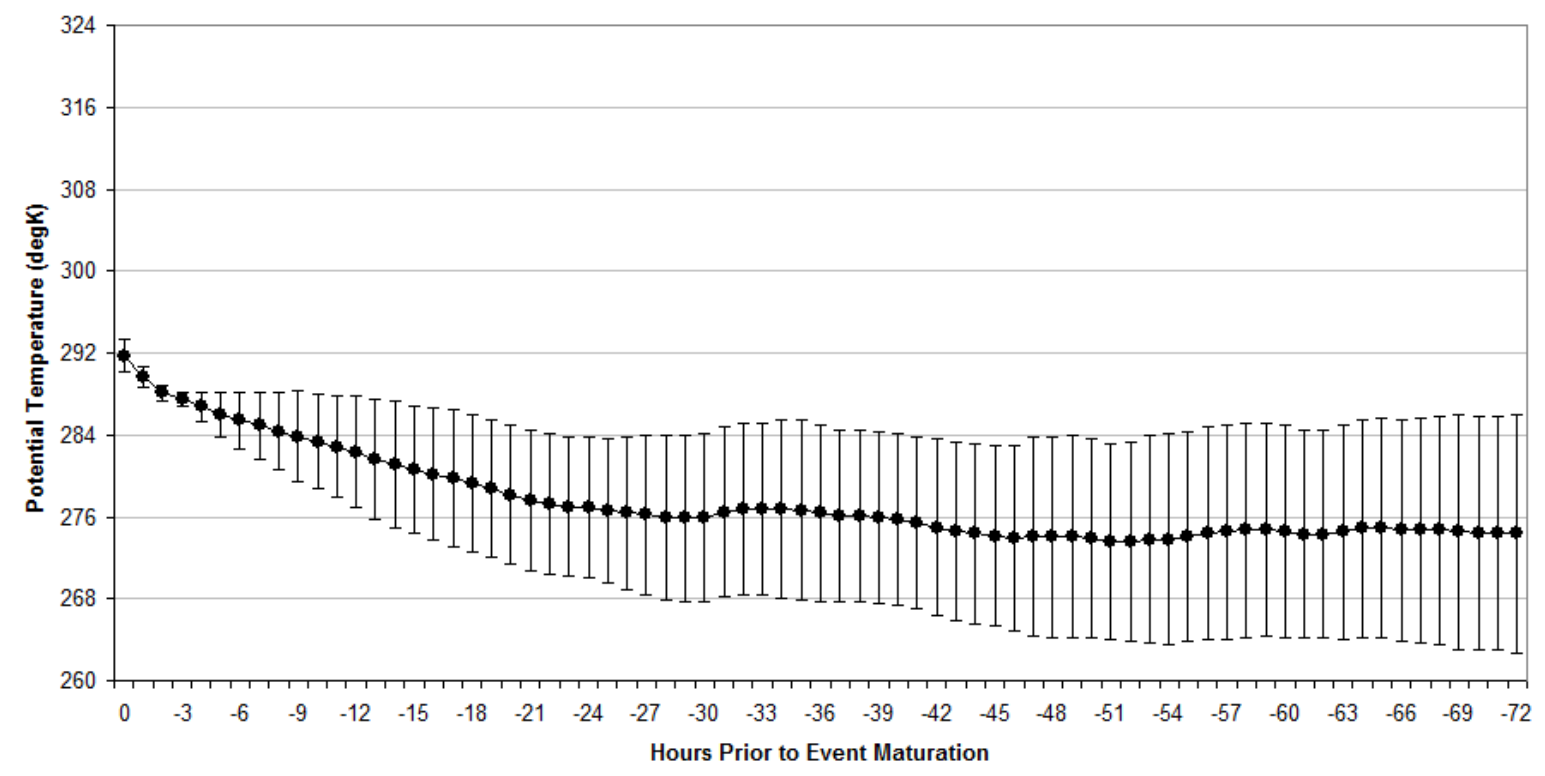

Figure A42. Composite warm layer zone trajectory of potential temperature with range bars.

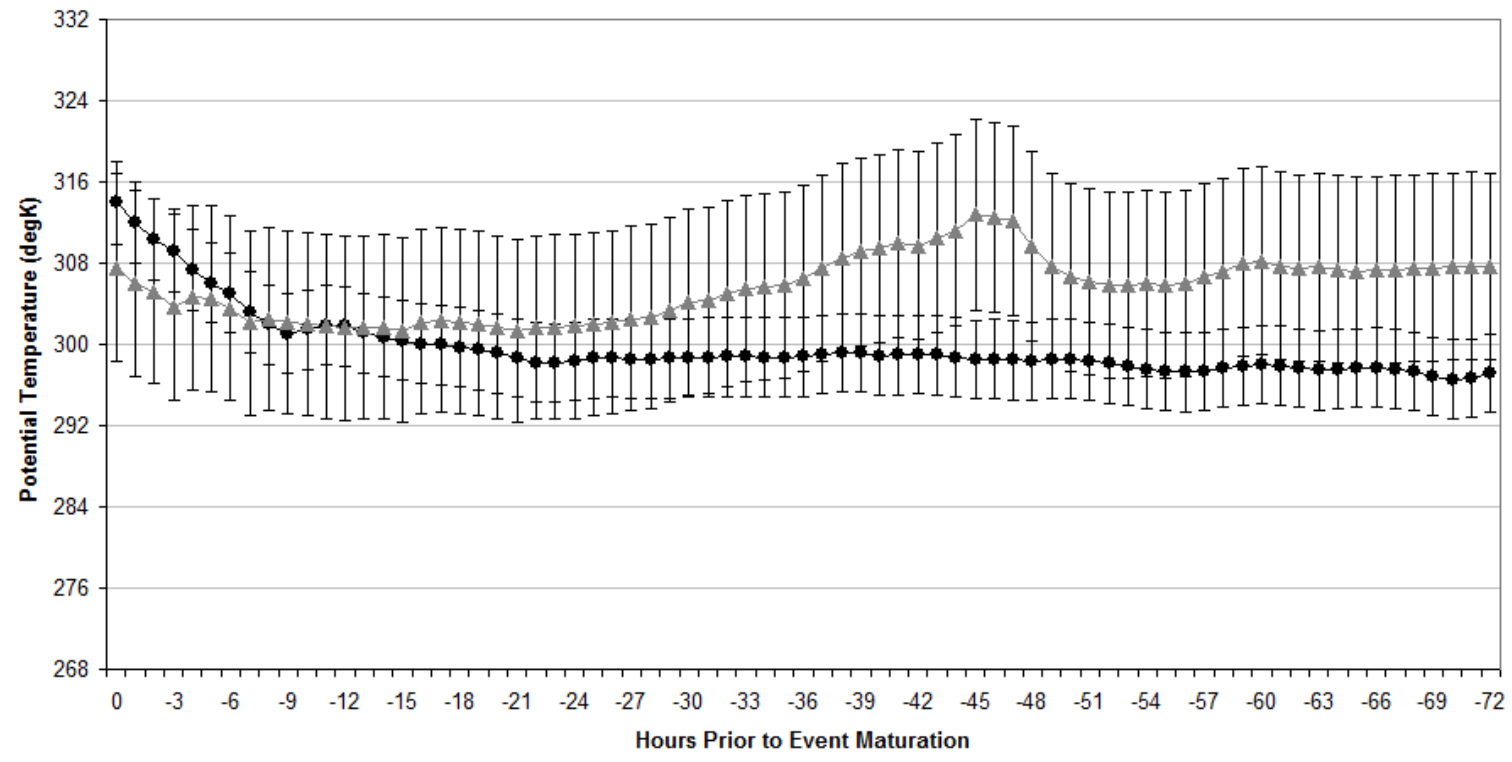

Figure A43. Composite DGZ trajectories of potential temperature with range bars. Circles represent the DGZ-anticyclonic trajectory and triangles represent the DGZ-cyclonic trajectory. 


\section{REFERENCES}

Appel, K.W., A.J. Riordan, and T.A. Holley, 2005: An objective climatology of Carolina coastal fronts. Weather and Forecasting, 20, 439-455.

Auer, A.H., and J.M. White, 1982: The combined role of kinematics, thermodynamics, and cloud physics associated with heavy snowfall episodes. Journal of the Meteorological Society of Japan, 60, 500-507.

Bailey, C.M., G. Hartfield, G.M. Lackmann, K.K. Keeter, and S. Sharp, 2003: An objective climatology, classification scheme, and assessment of sensible weather impacts for Appalachian cold-air damming. Weather and Forecasting, 18, 641-661.

Banacos, P. C., and M.L. Ekster, 2010: The association of the elevated mixed layer with significant severe weather events in the Northeastern United States. Weather and Forecasting, 25, 1082-1102.

Bane, J.M., and K.E. Osgood, 1989: Wintertime air-sea interaction processes across the Gulf Stream. Journal of Geophysical Research, 94, 10755-10772.

Bao, J.W., S.A. Michelson, P.J. Neiman, F.M. Ralph, and J.M. Wilczak, 2006: Interpretation of enhanced integrated water vapor bands associated with extratropical cyclones: Their formation and connection to tropical water vapor. Monthly Weather Review, 134, 10631080 .

Bell, G.D., and L.F. Bosart, 1988: Appalachian cold-air damming. Monthly Weather Review, 116, 137-161.

Bernstein, B.C., 2000: Regional and local influences on freezing drizzle, freezing rain, and ice pellet events. Weather and Forecasting, 15, 485-508.

Bierly, G.D., 1999: An investigation of the influence of cyclonic airstreams on Midwestern snowfall. Professional Geographer, 51, 340-349.

Bierly, G.D., and J.A. Winkler, 2001: A composite analysis of airstreams within cold-season Colorado cyclones. Weather and Forecasting, 16, 57-80.

Bjerknes, J., 1919: On the structure of moving cyclones. Geophysical Publications, 1, 1-8.

Black, T.L., 1994: The new NMC mesoscale Eta Model: Description and forecast examples. Weather and Forecasting, 9, 265-278.

Bocchieri, J.R., 1980: The objective use of upper air soundings to specific precipitation type. Monthly Weather Review, 108, 596-603. 
Brennan, M.J., and G.M. Lackmann, 2005: The influence of incipient latent heat release on the precipitation distribution of the 24-25 January 2000 U.S. East Coast cyclone. Monthly Weather Review, 133, 1913-1937.

Brimelow, J.C., and G.W. Reuter, 2005: Transport of atmospheric moisture during three extreme rainfall events over the Mackenzie River Basin. Journal of Hydrometeorology, 6 , 423-440.

Browning, K.A., 1990: Organization of clouds and precipitation in extratropical cyclones. Extratropical Cyclones, The Erik Palme'n Memorial Volume, C. W. Newton and E. O. Holopainen, Eds., Amer. Meteor. Soc., 129-153.

Browning, K.A., M. E. Hardman, T. W. Harrold, and C. W. Pardoe, 1973: The structure of rainbands within a mid-latitude depression. Quarterly Journal of the Royal Meteorological Society, 99, 215-231.

Carlson, T. N., 1980: Airflow through midlatitude cyclones and the comma cloud pattern. Monthly Weather Review, 108, 1498-1509.

Carr, F. H., and J. P. Millard, 1985: A composite study of comma clouds and their association with severe weather over the Great Plains. Monthly Weather Review, 113, $370-387$.

Changnon, S.A., 2004. Climate Atlas: Freezing Rain and Ice Storms. Changnon Climatologist, Mahomet, Illinois.

Changnon, S.A., 2003: Characteristics of ice storms in the United States. Journal of Applied Meteorology, 42, 630-639.

Changnon, S.A., 2003: Urban modification of freezing-rain events. Journal of Applied Meteorology, 42, 863-869.

Cortinas, J., 2000: A climatology of freezing rain in the Great Lakes region of North America. Monthly Weather Review, 128, 3574-3588.

Cram, T.A., J. Persing, M.T. Montgomery, and S.A. Braun, 2007: A lagrangian trajectory view on transport and mixing processes between the eye, eyewall, and environment using a high-resolution simulation of Hurricane Bonnie (1998). Journal of the Atmospheric Sciences, 64, 1835-1856.

Cuviello, M.P., 2007: A model for refining precipitation-type forecasts for winter weather in the Piedmont region of North Carolina on the basis of partial thickness and synoptic weather patterns. M.A. Thesis, Department of Geography, University of North Carolina at Chapel Hill, 97 pp. 
Czys, R.R., R.W. Scott, K.C. Tang, R.W. Przybylinski, and M.E. Sabones, 1996: A physically based, nondimensional parameter for discriminating between locations of freezing rain and sleet. Weather and Forecasting, 11, 591-598.

Danielson, E.F., 1964: Project Springfield Report. Defense Atomic Support Agency, DASA 1517, 97 pp. [NTIS AD-607980.]

Dayan, U., 1986: Climatology of back trajectories from Israel based on synoptic analysis. Journal of Climate and Applied Meteorology, 25, 591-595.

Dorling, S.R., T.D. Davies, and C.E. Pierce, 1992: Cluster analysis: a technique for estimating synoptic meteorological controls on air and precipitation chemistry - method and applications. Atmospheric Environment, 26, 2575-2581.

Draxler, R.R., 1991: The accuracy of trajectories during ANATEX calculated using dynamic model analysis versus rawinsonde observations. Journal of Applied Meteorology, 30, 1446-1467.

Draxler, R.R., 1992: Hybrid single-particle Lagrangian integrated trajectories (HY-SPLIT): Version 3.0 User's Guide and Model Description. NOAA Technical Memo. ERL-ARL195.

Draxler, R.R., 1996: Trajectory optimization for balloon flight planning. Weather and Forecasting, 11, 111-114.

Draxler, R.R., and B.J.B. Stunder, 1988: Modeling the CAPTEX vertical tracer concentration profiles. Journal of Applied Meteorology, 27, 617-625.

Draxler, R.R., and A.D. Taylor, 1982: Horizontal dispersion parameters for long-range transport modeling. Journal of Applied Meteorology, 21, 367-372.

Draxler, R., and G. Hess, 1998: An overview of the HYSPLIT_4 modeling system for trajectories, dispersion, and deposition. Australian Meteorological Magazine, 47, 295308.

Draxler, R.R. and Rolph, G.D., 2011. HYSPLIT (HYbrid Single-Particle Lagrangian Integrated Trajectory) Model access via NOAA ARL READY Website (http://ready.arl.noaa.gov/HYSPLIT.php). NOAA Air Resources Laboratory, Silver Spring, MD.

Draxler, R.R., 2003: Evaluation of an ensemble dispersion calculation. Journal of Applied Meteorology, 42, 308-317.

Dutton, J.A., 1986: The Ceasless Wind: An Introduction to the Theory of Atmospheric Motion. Dover, New York. 
Eckhardt, S., A. Stohl, H. Wernli, P. James, C. Forster, and N. Spichtinger, 2004: A 15-year climatology of warm conveyor belts. Journal of Climate, 17, 218-237.

Forbes, G.S., R.A. Anthes, and D.W. Thomson, 1987: Synoptic and mesoscale aspects of an Appalachian ice storm associated with cold-air damming. Monthly Weather Review, 115, 564-591.

Fritsch, J.M., and Coauthors, 1998: Meeting summary: Quantitative precipitation forecasts: Report of the Eighth Prospectus Development Team. U.S. Weather Research Program. Bulletin of the American Meteorological Society, 79, 285-299.

Fuhrmann, C.M., and C.E. Konrad II, 2005: A winter weather climatology for the Southeast United States. Available at http://www.nc-climate.ncsu.edu/climate/winter

Fuhrmann, C.M., R.P. Connolly, and C.E. Konrad, 2009: Winter storms: An overlooked source of death, destruction, and inconvenience in the Carolina Piedmont region. Proceedings of the Eastern Snow Conference, 66, 45-58.

Gay, D.A., and R.E. Davis, 1993: Freezing rain and sleet climatology of the southeastern USA. Climate Research, 3, 209-220.

Gurka, J. J., E. P. Auciello, A. F. Gigi, J. S. Waldstreicher, K. K. Keeter, S. Businger, and L. G. Lee, 1995: Winter weather forecasting throughout the eastern United States. Part II: An operational perspective of cyclogenesis. Weather and Forecasting, 10, 21-41.

Gustafsson, M., D. Rayner, and C. Deliang, 2010: Extreme rainfall events in southern Sweden: where does the moisture come from? Tellus, 62, 605-616.

Gyakum, J.R., and P.J. Roebber, 2001: The 1998 ice storm - analysis of a planetary-scale event. Monthly Weather Review, 129, 2983-2997.

Harrold, T. W., 1973: Mechanisms influencing the distribution of precipitation within baroclinic disturbances. Quarterly Journal of the Royal Meteorological Society, 99, 232251.

Heffter, J.L., and B.J.B. Stunder, 1993: Volcanic ash forecast transport and dispersion (VAFTAD) model. Weather and Forecasting, 8, 533-541.

Heppner, P.O.G., 1992: Snow versus rain: Looking beyond the "magic" numbers. Weather and Forecasting, 7, 678-691.

Holton, J.R., 2004: An Introduction to Dynamic Meteorology, $4^{\text {th }}$ ed., 535 pp., Elsevier, New York. 
Homan, J., and L.W. Uccellini, 1987: Winter forecast problems associated with light to moderate snow events in the mid-Atlantic states on 14 and 22 February 1986. Weather and Forecasting, 2, 206-228.

Hondula, D.M., L. Sitka, R.E. Davis, D.B. Knight, S.D. Gawtry, M.L. Deaton, T.R. Lee, C.P. Normile, and P.J. Stenger, 2009: A back-trajectory and air mass climatology for the Northern Shenandoah Valley, USA. International Journal of Climatology, 30, 569-581.

Hunter, S.M., S.J. Underwood, R.L. Holle, and T.L. Mote, 2001: Winter lightning and heavy frozen precipitation in the Southeast United States. Weather and Forecasting, 16, 478490.

James, P., A. Stohl, N. Spichtinger, S. Eckhardt, and C. Foster, 2004: Climatological aspects of the extreme European rainfall of August 2002 and a trajectory method for estimating the associated evaporative source regions. Natural Hazards and Earth Systems Science, 4, 733-746.

Jones, K.F., A.C. Ramsay, and J.N. Lott, 2004: Icing severity in the December 2002 freezing-rain storm from ASOS data. Monthly Weather Review, 132, 1630-1644.

Jorba, O., C. Perez, F. Rocadenbosch, and J. Baldasano, 2004: Cluster analysis of 4-day back trajectories arriving in the Barcelona Area, Spain, from 1997 to 2002. Journal of Applied Meteorology, 43, 887-901.

Kahl, J.D., and P.J. Samson, 1988: Trajectory sensitivity to rawinsonde data resolution. Atmospheric Environment, 22, 1291-1299.

Kain, J.S., S.M. Goss, and M.E. Baldwin, 2000: The melting effect as a factor in precipitation-type forecasting. Weather and Forecasting, 15, 700-714.

Keeter, K.K., and J.W. Cline, 1991: The objective use of observed and forecast thickness values to predict precipitation type in North Carolina. Weather and Forecasting, 6, 456469.

Keeter, K.K., S. Businger, and L.G. Lee, 1993: Patterns of winter precipitation types across North Carolina. Preprints, $13^{\text {th }}$ Conference on Weather Analysis and Forecasting, Vienna, VA, Amer. Meteor. Soc., 528-532.

Keeter, K.K., S. Businger, L.G. Lee, and J.S. Waldstreicher, 1995: Winter weather forecasting throughout the eastern United States. Part III: The effects of topography and the variability of winter weather in the Carolinas and Virginia. Weather and Forecasting, 10, 42-60.

Knippertz, P., and J.E. Martin, 2007: A Pacific water vapor conveyor belt and its relationship to a significant precipitation event in the semiarid southwestern United States. Weather and Forecasting, 22, 125-144. 
Kocin, P.J., and L.W. Uccellini, 2004: Northeast Snowstorms - Volume I: Overview. Meteorological Monograph No. 54, American Meteorological Society.

Lackmann, G.M., 2006: Improving cold-season quantitative precipitation forecasting in the southeastern United States. Final report on the proposal to NOAA in support of the Collaborative Science, Technology, and Applied Research Program (CSTAR). Available athttp://www4.ncsu.edu/ nwsfo/storage/resupdates/Revised_NCSU_CSTAR_II_Final_R eport.pdf

Lackmann, G.M., and W.M. Stanton, 2004: Cold-air damming erosion: Physical mechanisms, synoptic settings, and model representation. Preprints, $20^{\text {th }}$ Conference on Weather Analysis and Forecasting, Seattle, WA, Amer. Meteor. Soc., CD-ROM 18.6.

Lackmann, G.M., K.K. Keeter, L.G. Lee, and M.B. Ek, 2002: Model representation of freezing and melting precipitation: Implications for winter weather forecasting. Weather and Forecasting, 17, 1016-1033.

Liu, G., 1997: Case studies of water supply in oceanic extratropical cyclones using an Eulerian-Lagrangian method. Ph.D. dissertation, Department of Physics and Atmospheric Science, Drexel University, 212 pp.

Mahoney, K.M., and G.M. Lackmann, 2006: The effect of upstream convection on downstream precipitation. Weather and Forecasting, 22, 255-277.

Martin, J.E., 1998: The structure and evolution of a continental winter cyclone. Part II: Frontal forcing of an extreme snow event. Monthly Weather Review, 126, 329-348.

Martin, J. E., 1999: Quasigeostrophic forcing of ascent in the occluded sector of cyclones and the trowal airstream. Monthly Weather Review, 127, 70-88.

Martner, B.E., J.B. Snider, R.J. Zamora, G.P. Byrd, T.A. Niziol, and P.I. Joe, 1993: A remote-sensing view of a freezing-rain storm. Monthly Weather Review, 121, 2562-2577.

Maryon, R.H., and C.C. Heasman, 1988: The accuracy of plume trajectories forecast using the U.K. meteorological office operational forecasting models and their sensitivity to calculation schemes. Atmospheric Environment, 22, 259-272.

Mass, C.F., and D.M. Schultz, 1993: The structure and evolution of a simulated midlatitude cyclone over land. Monthly Weather Review, 121, 889-917.

Matsuo, T., and Y. Sayo, 1981: Melting of snowflakes below freezing level in the atmosphere. Journal of the Meteorological Society of Japan, 59, 10-25.

Mesinger, F., and Coauthors, 2006: North American Regional Reanalysis. Bulletin of the American Meteorological Society, 87, 343-360. 
Miller, J.E., 1946: Cyclogenesis in the Atlantic Coastal region of the United States. Journal of Meteorology, 3, 31-44.

Milrad, S.M., E.H. Atallah, and J.R. Gyakum, 2010: Synoptic typing of extreme cool-season precipitation events at St. John's, Newfoundland, 1979-2005. Weather and Forecasting, 25, 562-586.

Moore, J.T., S. Ng, and C.E. Graves, 2005: The role of conveyor belts in organizing processes associated with heavy banded snowfall. Preprints, $21^{\text {st }}$ Conference on Weather Analysis and Forecasting, Washington, D.C., Amer. Meteor. Soc., 10A.1

Mote, T.L., D.W. Gamble, S.J. Underwood, and M.L. Bentley, 1997: Synoptic-scale features common to heavy snowstorms in the Southeast United States. Weather and Forecasting, 12, 5-23.

Moyer, B.W., 2001: A record southeastern snowfall in the absence of a rapidly deepening extratropical cyclone. NOAA Eastern Technical Attachment No. 2001-02. http://www.erh.noaa.gov/gsp/localdat/ta2001-02.pdf

Ng, S., 2005: Development of a dynamical conceptual model of processes producing heavy banded snowfall utilizing numerical simulations. Ph.D. Dissertation, Department of Atmospheric Science, St. Louis University, 343 pp.

Nicosia, D.J., and R.H. Grumm, 1999: Mesoscale band formation in three major northeastern United States snowstorms. Weather and Forecasting, 14, 346-368.

Penner, C.M., 1955: A three-front model for synoptic analyses. Quarterly Journal of the Royal Meteorological Society, 81, 89-91.

Perry, L.B., 2006: Synoptic climatology of northwest flow snowfall in the Southern Appalachians. Ph.D. Dissertation, Department of Geography, University of North Carolina at Chapel Hill, 192 pp.

Petterssen, S., 1940: Weather Analysis and Forecasting. McGraw-Hill Book Co., New York.

Raman, S., A.J. Riordan, T. Holt, M. Stinder, and J. Hinman, 1986: Observations of the marine boundary layer thermal structure over the Gulf Stream during a cold air outbreak. Journal of Climate and Applied Meteorology, 25, 14-21.

Ramos Da Silva, R., G. Bohrer, D. Werth, M.J. Otte, and R. Avissar, 2006: Sensitivity of ice storms in the southeastern United States to Atlantic SST - Insights from a case study of the December 2002 storm. Monthly Weather Review, 134, 1454-1464. 
Raynor, G.S., J.V. Hayes, and D.M. Lewis, 1983: Testing of the Air Resources Laboratories trajectory model on cases of pollen wet deposition after long-distance transport from known source regions. Atmospheric Environment, 17, 213-220.

Reap, R.M., 1972: An operational three-dimensional trajectory model. Journal of Applied Meteorology, 11, 1193-1202.

Richwein, B.A., 1980: The damming effect of the southern Appalachians. National Weather Digest, 5, 2-12.

Robbins, C.C., and J.V. Cortinas, 2002: Local and synoptic environments associated with freezing rain in the contiguous United States. Weather and Forecasting, 17, 47-65.

Roberge, A., J. Gyakum, and E. Atallah, 2009: Analysis of intense poleward water vapor transports into high latitudes of western North America. Weather and Forecasting, 24, $1732-1747$.

Roebber, P.J., and L.F. Bosart, 1998: The sensitivity of precipitation to circulation details, Part I: An analysis of regional analogs. Monthly Weather Review, 126, 437-455.

Rogers, E., T. Black, B. Ferrier, Y. Lin, D. Parrish, and G. DiMego, 2001: Changes to the NCEP Meso Eta Analysis and Forecast System: Increase in resolution, new cloud microphysics, modified precipitation assimilation, modified 3DVAR analysis. NWS Technical Procedures Bull. 479, 13 pp.

Rolph, GD, 2011: Real-time Environmental Applications and Display System (READY) website: http://ready.url.noaa.gov/. NOAA’s Air Resources Laboratory, Silver Spring, MD.

Schultz, D.M., 2001: Reexamining the cold conveyor belt. Monthly Weather Review, 129, 2205-2225.

Schultz, D.M., J.V. Cortinas, and C.A. Doswel, 2002: Comments on “An operational ingredients-based methodology for forecasting midlatitude winter season precipitation". Weather and Forecasting, 17, 160-167.

Stewart, R.E., 1985: Precipitation types in winter storms. Pure and Applied Geophysics, 123, 597-609.

Stewart, R.E., 1992: Precipitation types in the transition region of winter storms. Bulletin of the American Meteorological Society, 73, 287-296.

Stohl, A., and P. Seibert, 1997: Accuracy of trajectories as determined from the conservation of meteorological tracers. Quarterly Journal of the Royal Meteorological Society, 
Stohl, A., and P. Seibert, 1998: Accuracy of trajectories as determined from the conservation of meteorological tracers. Quarterly Journal of the Royal Meteorological Society, 125, 1465-1484.

Stohl, A., L. Haimberger, M.P. Scheele, and H. Wernli, 2001: An intercomparison of results from three trajectory models. Meteorological Applications, 8, 127-135.

Stohl, A., 1998: Computation, accuracy and applications of trajectories - a review and bibliography. Atmospheric Environment, 32, 947-966.

Uccellini, L.W., D. Keyser, K.F. Brill, and C.H. Wash, 1985: The Presidents' Day cyclone of 18-19 February 1979: Influence of upstream trough amplification and associated tropopause folding on rapid cyclogenesis. Monthly Weather Review, 115, 2227-2261.

Wagner, J.A., 1957: Mean temperature from $1000 \mathrm{mb}$ to $500 \mathrm{mb}$ as a predictor of precipitation type. Bulletin of the American Meteorological Society, 38, 584-590.

Waldstreicher, J.S., 2005: Assessing the impact of collaborative research projects on NWS warning performance. Bulletin of the American Meteorological Society, 86, 193-203.

Wandishin, M.S., M.E. Baldwin, S.L. Mullen, and J.V. Cortinas, 2005: Short-range ensemble forecasts of precipitation type. Weather and Forecasting, 20, 609-626.

Wernli, H., 1997: A lagrangian-based analysis of extratropical cyclones II: A detailed case study. Quarterly Journal of the Royal Meteorological Society, 123, 1677-1706.

Wernli, H., and H.C. Davies, 1997: A lagrangian-based analysis of extratropical cyclones I: The method and some applications. Quarterly Journal of the Royal Meteorological Society, 123, 467-489.

Wetzel, S.W., and J.E. Martin, 2001: An operational ingredients-based methodology for forecasting midlatitude winter season precipitation. Weather and Forecasting, 16, 156167.

White, A.B., and Co-authors, 2006: A wind profiler trajectory tool for air quality transport applications. Journal of Geophysical Research, doi:10.1029/2006JD007475.

Zerr, R.J., 1997: Freezing rain: An observational and theoretical study. Journal of Applied Meteorology, 36, 1647-1661. 